\title{
The burden of neurosarcoidosis and small fiber neuropathy associated symptoms
}

Citation for published version (APA):

Voortman, M. (2019). The burden of neurosarcoidosis and small fiber neuropathy associated symptoms. [Doctoral Thesis, Maastricht University]. ProefschriftMaken Maastricht. https://doi.org/10.26481/dis.20191129mv

Document status and date:

Published: 01/01/2019

DOI:

10.26481/dis.20191129mv

Document Version:

Publisher's PDF, also known as Version of record

\section{Please check the document version of this publication:}

- A submitted manuscript is the version of the article upon submission and before peer-review. There can be important differences between the submitted version and the official published version of record.

People interested in the research are advised to contact the author for the final version of the publication, or visit the DOI to the publisher's website.

- The final author version and the galley proof are versions of the publication after peer review.

- The final published version features the final layout of the paper including the volume, issue and page numbers.

Link to publication

\footnotetext{
General rights rights.

- You may freely distribute the URL identifying the publication in the public portal. please follow below link for the End User Agreement:

www.umlib.nl/taverne-license

Take down policy

If you believe that this document breaches copyright please contact us at:

repository@maastrichtuniversity.nl

providing details and we will investigate your claim.
}

Copyright and moral rights for the publications made accessible in the public portal are retained by the authors and/or other copyright owners and it is a condition of accessing publications that users recognise and abide by the legal requirements associated with these

- Users may download and print one copy of any publication from the public portal for the purpose of private study or research.

- You may not further distribute the material or use it for any profit-making activity or commercial gain

If the publication is distributed under the terms of Article $25 \mathrm{fa}$ of the Dutch Copyright Act, indicated by the "Taverne" license above, 
The burden of neurosarcoidosis and small fiber neuropathy associated symptoms 
${ }^{\circledR}$ Copyright Mareye Voortman, Utrecht, 2019

$\begin{array}{ll}\text { Lay out } & \text { Tiny Wouters } \\ \text { Cover photo } & \text { Petal Wijnen } \\ \text { Cover design } & \text { NPN Communicatie, Breda } \\ \text { Print } & \text { ProefschriftMaken }\end{array}$

ISBN/EAN 978-90-78076-11-7

The research published in this thesis was financially supported by ZonMw, Sarcoidose.nl and the ild care foundation.

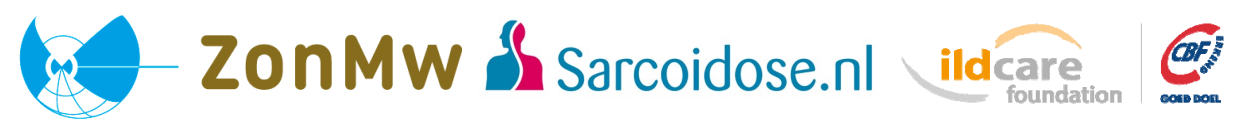

The printing of this thesis was financially supported by Boehringer Ingelheim, Chiesi, iDoctor, ILD center of Excellence St. Antonius Hospital Nieuwegein, and the ild care foundation.

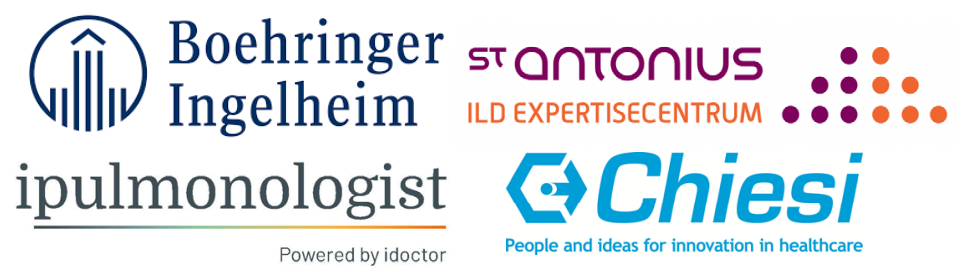




\title{
The burden of neurosarcoidosis and small fiber neuropathy associated symptoms
}

\author{
PROEFSCHRIFT
}

ter verkrijging van de graad van doctor aan de Universiteit Maastricht, op gezag van de Rector Magnificus, Prof.dr. Rianne M. Letschert volgens het besluit van het College van Decanen, in het openbaar te verdedigen op vrijdag 29 november 2019 om 14.00 uur

door

Mareye Voortman 


\section{Promotores:}

Prof. dr. M. Drent

Prof. dr. J. De Vries (Universiteit Tilburg)

\section{Beoordelingscommissie:}

Prof. dr. F.W.J.M. Smeenk (voorzitter)

Prof. dr. A. Bast

Prof. dr. A. Dahan (Universiteit Leiden)

Prof. dr. J.C. Grutters (Universiteit Utrecht)

Prof. dr. R.W.H.M. Ponds 


\section{Contents}

$\begin{array}{lll}\text { Chapter } 1 \quad \text { General introduction } & 7\end{array}$

$\begin{array}{lr}\text { Part I } & 19\end{array}$

Chapter 2 Many faces of neurosarcoidosis: from chronic meningitis 21 to myelopathy

Chapter 3 Clinical manifestations of neurosarcoidosis in the 37 Netherlands

Chapter 4 Everyday cognitive failure in patients suffering from neurosarcoidosis

Chapter 5 Management of neurosarcoidosis: a clinical challenge

Part II

85

Chapter 6 Small fiber neuropathy: a disabling and under recognized syndrome

Chapter 7 Determination of the smallest detectable change (SDC) and the minimal important difference (MID) for the Small Fiber Neuropathy Screening List (SFNSL) in sarcoidosis. Interpretability of the SFNSL

Chapter 8 The burden of sarcoidosis symptoms from a patients' perspective

Chapter 9 Quality of life of couples living with sarcoidosis

Chapter 10 Summary, general discussion and directions for future research

Part III

Valorisatie

Samenvatting (summary in Dutch)

List of publications

Dankwoord

Curriculum vitae

Abbreviations

Appendix questionnaires: vragenlijsten (FAS, SFNSL, CFQ) 



\section{Chapter 1}

General introduction 



\section{General introduction}

Sarcoidosis is a multisystem inflammatory disorder of unknown etiology. The disease is characterized by the formation of non-caseating granulomas in various organ systems, mainly the lungs and lymphatic system.,

\section{Epidemiology}

The incidence of sarcoidosis is approximately 1-40 per 100000 persons. Sarcoidosis occurs in both women and men, with a slight predominance in women. It can affect people of all ages, but predominantly between $25-45$ years. ${ }^{3}$ Sarcoidosis occurs all over the world, but the incidence and prevalence of sarcoidosis in general as well as of different sarcoidosis manifestations differs in various countries due to genetic and environmental differences. ${ }^{4}$ For example, cardiac sarcoidosis is more common in Japan, ${ }^{5,6}$ while Lofgren syndrome (an acute variant with fever, arthralgia, erythema nodosum, and bilateral hilar lymphadenopathy) is more common in Scandinavian countries. $^{7-9}$

The natural history and prognosis of sarcoidosis are highly variable. ${ }^{1}$ Within 3 years of diagnosis, more than $50 \%$ of patients have achieved remission, and after a decade, approximately one-third of patients have persistent disease, leading to a significant burden on their lives. ${ }^{10}$ Several patient characteristics, like African-American descent, advanced pulmonary disease, extrapulmonary involvement, uveitis, hypercalcemia, and lupus pernio are associated with a chronic course of sarcoidosis. ${ }^{11}$ Moreover, genetic variants (HLA-DRB1*14 and *15) tend to increase the risk of a more progressive course of the disease and of non-resolving disease. ${ }^{9}$

\section{Pathogenesis}

The exact cause of sarcoidosis is still unknown, but is likely to depend on both genetic and environmental factors, probably antigen-driven. It involves an interaction between a putative triggering antigen, probably inhaled, and a genetically susceptible host (specific HLA-genotypes) resulting in an exaggerated T-cell-mediated immune response. $^{12}$ Different antigens/environmental factors have been identified, such as infection (mycobacterial, Propionibacterium acnes), ${ }^{13-20}$ insecticides, pesticides, organic/wood/metal dust, and mold. ${ }^{21,22}$

Hallmark features of sarcoidosis are well-developed granulomas, consisting of macrophages (fusing to form multinucleated giant cells) and epithelioid cells surrounded by lymphocytes, especially CD4+ T-helper (Th) cells. ${ }^{23}$ Macrophages play a fundamental role, for instance as antigen-presenting cells (APCs). The antigen is 
ingested by the APC, inserted in the antigen-binding groove of a major histocompatibility complex ( $\mathrm{MHC}$ ) class II molecule, and transported to the surface for presentation to the T-cells (see figure 1.1). ${ }^{24}$ The interaction between macrophages and T-cells is essential for T-cell activation. ${ }^{10}$ Activation of antigen-specific CD4+ T-cells by MHC class II restricted APCs leads to a type 1 helper (Th1) differentiation of CD4+ Tcells. Th1-cells then release increased amounts of interferon-gamma (IFN- $\gamma$ ) and interleukin-2 (IL-2). IL2 acts as a local growth factor for T-lymphocytes. IFN- $\gamma$ enhances the accessory and cytotoxic functions of T-cells and regulates the secretion of other lymphokines (IL-12, IL-18, and tumour necrosis factor-alpha [TNF- $\alpha$ ]), which in turn will induce IFN- $\gamma$ production and enhance T-cell toxicity. ${ }^{10,22}$ Moreover, IFN- $\gamma$ amplifies the activation of alveolar macrophages, leading to increased release of nuclear factor (NF)kB-dependent proinflammatory cytokines, such as several interleukins and TNF- $\alpha{ }^{25}$ TNF- $\alpha$ is an important granuloma-promoting factor in sarcoidosis. This immune response induces granuloma formation and maintenance.
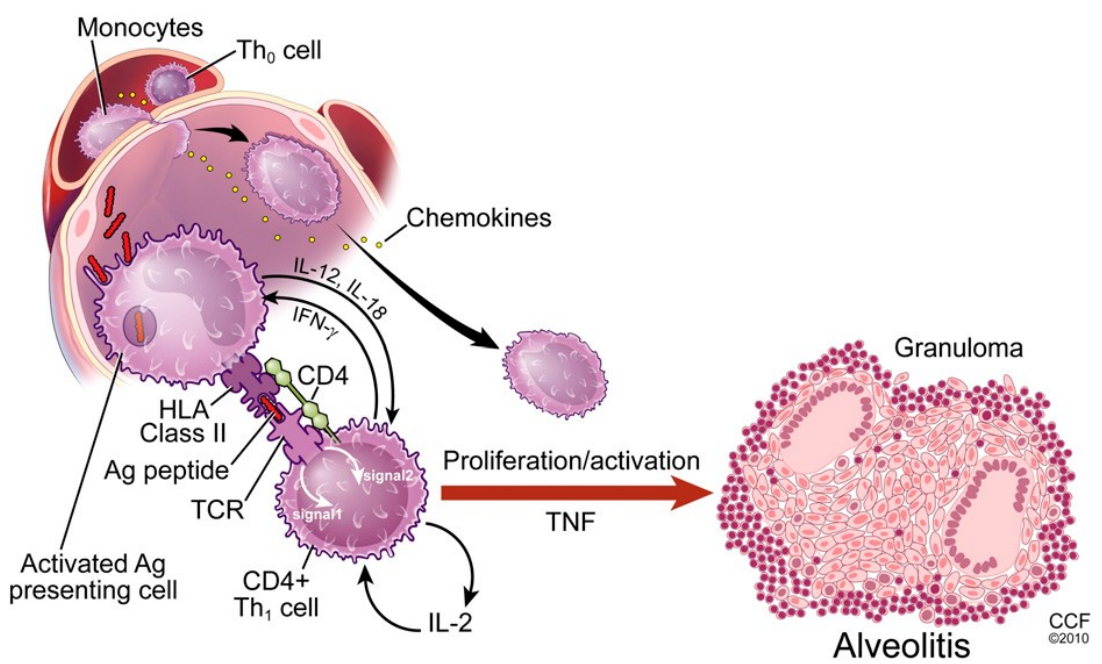

Figure 1.1 A schematic representation of granuloma formation in sarcoidosis.

Th=T-helper; Ag=antigen; HLA=human leukocyte antigen; IFN- $\gamma=$ interferon-gamma; $\mathrm{IL}=$ interleukin; TCR=T-cell receptor; TNF=tumour necrosis factor (adapted from Baughman). ${ }^{26}$ Hypothetical model of the pathogenesis of sarcoidosis, an antigen-induced, antigen-specific, Th1-mediated granulomatous inflammation with production of Th1 cytokines (IFN- $\gamma$, IL-2). The efficiency of antigen processing, antigen presentation, and cytokine release is probably under genetic control; evidence strongly supports a role for macrophage HLA and butyrophilin-like 2 (BTNL2) alleles in sarcoidosis susceptibility and phenotype. Granuloma formation is set in motion by activated macrophages and T-cells along with other effector cells (e.g. fibroblasts) under the regulatory influence of local cytokine production. Removal of the antigen allows transforming growth factor beta (TGF- $\beta$ ) to downregulate the immune response. Alveolar macrophages activated in the context of a predominant Th2 response appear to stimulate fibroblast proliferation and collagen production, leading to progressive fibrosis. ${ }^{3}$ 


\section{Neurosarcoidosis}

The clinical presentation of sarcoidosis is highly variable and its course unpredictable. Clinical manifestations vary, depending on the organs involved. All organs can be affected, but the most commonly affected organs are the lymphatic system and the lungs. ${ }^{2}$ Involvement of the nervous system, neurosarcoidosis, is present in approximately $5-15 \%$ of patients with sarcoidosis. ${ }^{2,27}$ However, autopsy studies have found granulomatous inflammation of the nervous system in up to $25 \%$ of patients. ${ }^{28}$ It is a rare and serious sarcoidosis manifestation, which often requires treatment. Neurosarcoidosis has a heterogeneous clinical presentation, including cranial and peripheral neuropathy, meningitis, hydrocephalus, cerebral lesions, and spinal cord disease (see figures 1.2 and 1.3 ). ${ }^{29}$

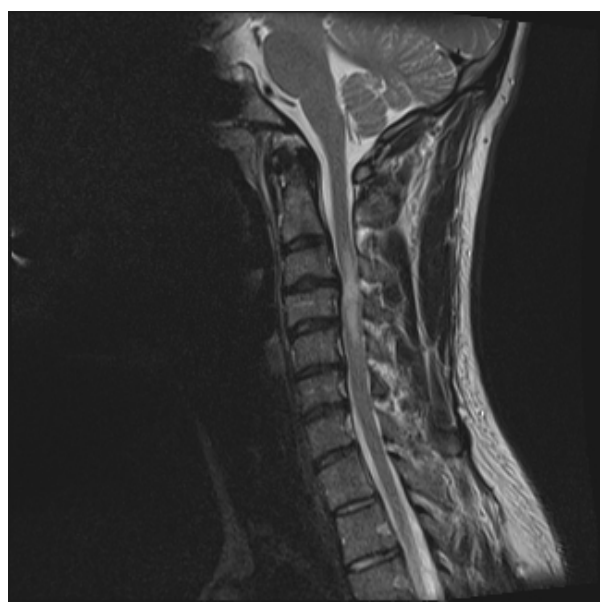

Figure 1.2 36-year-old male, presenting with numbness in both arms and cervical pain. The MRI showed a cervical myelitis (C3-C5). A chest X-ray showed bilateral mediastinal lymphadenopathy, and the diagnosis of sarcoidosis was confirmed with endobronchial ultrasound fine-needle aspiration (EBUS-FNA).

The range of clinical manifestations and the fact that neurosarcoidosis can be the first presenting symptom of sarcoidosis make it hard to diagnose this disorder. Previous literature has predominantly reported on retrospective studies. Since neurosarcoidosis is a rare, complex, and serious manifestation of sarcoidosis, more research into this disease entity is warranted (see also chapters 2 and 5). 


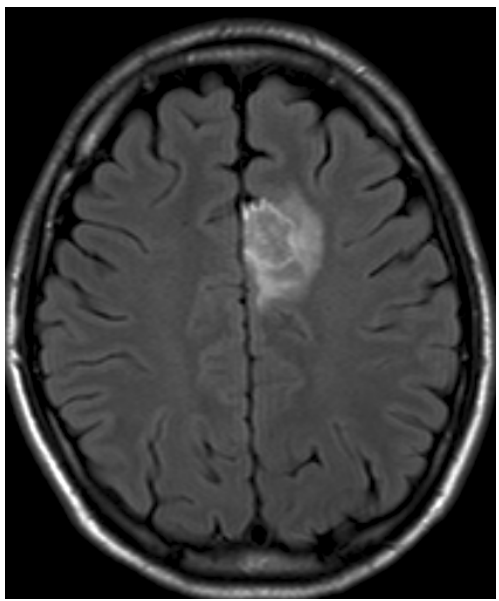

Figure 1.3 23-year-old male, presenting with epilepsy. The MRI showed an intracerebral lesion. A PET-CT demonstrated mediastinal lymph node and pulmonary involvement compatible with sarcoidosis. The diagnosis of sarcoidosis was confirmed with endobronchial ultrasound fineneedle aspiration (EBUS-FNA).

\section{Non-specific symptoms}

Apart from organ-related symptoms, sarcoidosis patients often suffer from a variety of nonspecific, non-organ-related, disabling symptoms that cannot be explained by granulomatous inflammation of an organ, such as fatigue, symptoms associated with small fiber neuropathy (SFN), and cognitive failure.

Fatigue is the most frequently reported and devastating non-specific symptom, with a reported prevalence ranging from 50 to $90 \%{ }^{30,31}$ Although it is globally recognized as a disabling symptom, no organic substrate has been found for sarcoidosis-associated fatigue. Its etiology is poorly understood and is likely to be multifactorial. ${ }^{32}$ In order to objectify fatigue in sarcoidosis patients, the fatigue assessment scale (FAS) has been developed. The FAS has been shown to be an easy-to-use, reliable, and valid scale for the assessment of fatigue, and it has good psychometric properties. ${ }^{33,34}$

Another substantial problem in sarcoidosis patients is SFN, with an estimated prevalence of $40-60 \% .{ }^{35}$ Symptoms associated with SFN are disabling and probably underrecognized, causing patients to feel misunderstood and imposing limitations on daily life activities. It is a generalized sensory nerve disorder with structural and functional abnormalities of small fibers. It is characterized histopathologically by degeneration of small fiber nerve endings, selectively involving thin myelinated Ad-and unmyelinated C-fibers. ${ }^{36}$ Small fibers are associated with thermal and nociceptive 
sensations (somatic) and autonomic function, resulting in symptoms of neuropathic pain and autonomic dysfunction. ${ }^{35,37}$ A screening tool for SFN-associated symptoms has been developed for patients with sarcoidosis, called the SFN screening list (SFNSL), ${ }^{38}$ which was intended as a first attempt to achieve early identification of patients with symptoms related to SFN (see also chapter 6).

Everyday cognitive failure, including concentration problems, memory loss, and decreased perception, was found in $35 \%$ of patients in a general sarcoidosis population. ${ }^{39}$ Cognitive failure can lead to difficulties in managing the disease and can adversely affect the treatment. ${ }^{40}$ Everyday cognitive failure and depressive symptoms are the most important predictors of high levels of fatigue, ${ }^{41}$ whereas other variables (time since diagnosis, sex, age, and social support) appeared not to predict fatigue.

All these non-specific symptoms account for many problems in the daily lives of sarcoidosis patients, leading to a high burden of disease and an important impact on their quality of life (QoL). ${ }^{42,43}$

\section{Treatment}

\section{Neurosarcoidosis}

The general purpose of systemic sarcoidosis treatment is to prevent end-organ damage. Decisions on whether to start systemic immunosuppressive treatment depend on the severity of the disease, e.g. the involvement and function of organs, or, in selected cases, on its impact on QoL. No curative treatment exists, only suppression of the disease. There are three lines of therapy for sarcoidosis. Corticosteroids are still the first-line treatment. For patients intolerant to corticosteroids, methotrexate (MTX) or azathioprine are well-established second-line options. Third-line treatment consists of anti-TNF antibodies, and is reserved for patients non-responsive to first- or second-line treatment. $^{44}$

Although some patients require no systemic treatment, treatment is almost always warranted in neurosarcoidosis. The intensity of treatment depends on the severity of the neurosarcoidosis manifestations. For example, a facial nerve paralysis can mostly be treated with prednisone monotherapy or no treatment at all, while for spinal cord disease one will choose a second-line (MTX, azathioprine, mycophenolate mofetil (MMF)) or third-line (TNF- $\alpha$ inhibitors; infliximab and adalimumab or more recently biosimilars) agent earlier on in the treatment of this manifestation. Limited data is available on the best treatment for neurosarcoidosis, and is mainly based on expert opinion and small retrospective studies. No randomized controlled trials have been performed comparing treatments for neurosarcoidosis. MTX seems to significantly 
increase the survival time without relapse, compared to MMF, and is therefore preferred. ${ }^{45}$ In another study, most patients treated with a TNF- $\alpha$ inhibitor, infliximab, responded with partial or complete remission. ${ }^{46}$ Further studies are needed to evaluate the prognosis and optimal treatment strategy (see also chapter 5).

\section{Small fiber neuropathy}

Currently, there is no curative treatment for SFN. Symptomatic treatment for neuropathic pain can be initiated, but unfortunately these drugs often provide only partial relief from pain, have no effect on autonomic dysfunction and are associated with (sometimes severe) side effects in a high proportion of patients. Some data suggest effectiveness of immunoglobulins and TNF- $\alpha$ inhibition. ${ }^{47-50}$ Whether these very expensive treatments should be initiated as treatment for SFN is unclear and currently being investigated. Cibinetide ${ }^{\circledR}$ (ARA290) seems a promising new drug to relieve pain and increase corneal and skin nerve-fiber density in sarcoidosis-associated SFN. ${ }^{51-53}$ Further clinical trials are needed before this drug can be authorized (see also chapter 6).

\section{Fatigue}

Despite effective treatment of their sarcoidosis with immunosuppressive agents, many patients continue to experience fatigue even after contributing factors (e.g. vitamin $D$ deficiency, hypothyroidism, or obstructive sleep apnea) have been treated. Recent studies have demonstrated the effectiveness of various neurostimulants, including methylphenidate, for the treatment of sarcoidosis-associated fatigue, and these and other agents may be useful adjuncts to its treatment. ${ }^{54}$ Moreover, it has been shown that muscle impairment is associated with fatigue and functional limitations. ${ }^{31,55}$ Symptoms of fatigue induce exercise limitation and lead to physical inactivity, resulting in general deconditioning. ${ }^{43}$ Several studies have reported that a physical training program can reduce fatigue and improve exercise capacity. ${ }^{32,56-58}$

\section{Scopes and aims of the studies}

The aims of the studies presented in this thesis were to outline the manifestations of neurosarcoidosis and the burden and/or impact of this disorder, such as cognitive impairment. In addition, they assessed the burden of sarcoidosis, focusing on fatigue and SFN-associated symptoms reported by the patients themselves. The third study determined the minimal important difference on the SFNSL instrument, which makes it possible to follow-up the SFN-related complaints experienced by patients. Finally, 
predictors of the QoL of patients and their partners (mainly concerning non-specific, non-organ-related complaints) were studied.

Chapter 2 provides an overview of the current literature regarding neurosarcoidosis. Clinical characteristics and various ancillary investigations are outlined, and treatment options and prognosis are discussed.

Chapter 3 describes the neurosarcoidosis manifestations in a large cohort of neurosarcoidosis patients in the Netherlands (obtained from the Amsterdam University Medical Centre, the ILD Center of Excellence, St. Antonius Hospital Nieuwegein, and the neurosarcoidosis registry). In addition, race and gender differences were studied and treatment options are described.

Chapter 4 discusses the high cognitive failure rate in neurosarcoidosis patients compared to the general sarcoidosis population. Many patients in the sample we studied experienced cognitive failure in their everyday lives.

Chapter 5 provides an overview of current literature regarding the management of neurosarcoidosis, including SFN, mainly focusing on diagnosing and treating neurosarcoidosis and SFN in sarcoidosis.

Chapter 6 summarizes the current literature regarding SFN, describing its pathophysiology and symptoms. The problems faced in diagnosing SFN are discussed, including the different diagnostic tests available. Various treatment options are presented.

Chapter 7 describes the determination of the minimal important difference (MID) on the SFNSL instrument. The MID is the smallest change in a score that a patient perceives to be important. For evaluative purposes, interpretability was assessed by the change in scores.

Chapter $\mathbf{8}$ describes the burden of sarcoidosis symptoms from a patient's perspective. This study was conducted in Denmark, Germany, and the Netherlands. Organ-related as well as non-specific, non-organ-related symptoms were assessed, as were treatment options.

Chapter 9 describes the QoL in sarcoidosis patients and their partners, as well as predictors of the QoL of patients and partners.

Chapter $\mathbf{1 0}$ provides a summary and general discussion of the findings reported on in this thesis. Additionally, the implications of the study outcomes for clinical practice and suggestions for future research are briefly discussed. 


\section{References}

1. Statement on sarcoidosis. Joint Statement of the American Thoracic Society (ATS), the European Respiratory Society (ERS) and the World Association of Sarcoidosis and Other Granulomatous Disorders (WASOG) adopted by the ATS Board of Directors and by the ERS Executive Committee, February 1999. Am J Respir Crit Care Med 1999;160(2):736-55.

2. Valeyre D, Prasse A, Nunes H, et al. Sarcoidosis. Lancet 2014;383(9923):1155-67.

3. Grunewald J, Grutters JC, Arkema EV, et al. Sarcoidosis. Nat Rev Dis Primers 2019;5(1):45.

4. Spagnolo P, Rossi G, Trisolini R, et al. Pulmonary sarcoidosis. Lancet Respir Med 2018;6(5):389-402.

5. Iwai $K$, Sekiguti $M$, Hosoda $Y$, et al. Racial difference in cardiac sarcoidosis incidence observed at autopsy. Sarcoidosis 1994;11(1):26-31.

6. Morimoto T, Azuma A, Abe S, et al. Epidemiology of sarcoidosis in Japan. Eur Respir J 2008;31(2):372-9.

7. Brown F, Tanner LS. Lofgren Syndrome. StatPearls. Treasure Island (FL)2018.

8. Darlington P, Gabrielsen A, Sorensson P, et al. HLA-alleles associated with increased risk for extrapulmonary involvement in sarcoidosis. Tissue Antigens 2014;83(4):267-72.

9. Grunewald J, Brynedal B, Darlington P, et al. Different HLA-DRB1 allele distributions in distinct clinical subgroups of sarcoidosis patients. Respir Res 2010;11:25.

10. Iannuzzi MC, Fontana JR. Sarcoidosis: clinical presentation, immunopathogenesis, and therapeutics. JAMA 2011;305(4):391-9.

11. Lazar CA, Culver DA. Treatment of sarcoidosis. Semin Respir Crit Care Med 2010;31(4):501-18.

12. Culver DA. Sarcoidosis. Immunol Allergy Clin North Am 2012;32(4):487-511.

13. Dubaniewicz A, Trzonkowski P, Dubaniewicz-Wybieralska M, et al. Mycobacterial heat shock proteininduced blood T lymphocytes subsets and cytokine pattern: comparison of sarcoidosis with tuberculosis and healthy controls. Respirology 2007;12(3):346-54.

14. Ebe $Y$, Ikushima S, Yamaguchi T, et al. Proliferative response of peripheral blood mononuclear cells and levels of antibody to recombinant protein from Propionibacterium acnes DNA expression library in Japanese patients with sarcoidosis. Sarcoidosis Vasc Diffuse Lung Dis 2000;17(3):256-65.

15. Eishi Y. Etiologic link between sarcoidosis and Propionibacterium acnes. Respir Investig 2013;51(2): 56-68.

16. Eishi Y. Etiologic aspect of sarcoidosis as an allergic endogenous infection caused by Propionibacterium acnes. Biomed Res Int 2013;2013:935289.

17. Ishige I, Usui Y, Takemura T, Eishi Y. Quantitative PCR of mycobacterial and propionibacterial DNA in lymph nodes of Japanese patients with sarcoidosis. Lancet 1999;354(9173):120-3.

18. Oswald-Richter KA, Beachboard DC, Seeley EH, et al. Dual analysis for mycobacteria and propionibacteria in sarcoidosis BAL. J Clin Immunol 2012;32(5):1129-40.

19. Oswald-Richter KA, Culver DA, Hawkins C, et al. Cellular responses to mycobacterial antigens are present in bronchoalveolar lavage fluid used in the diagnosis of sarcoidosis. Infect Immun 2009;77(9):3740-8.

20. Song Z, Marzilli L, Greenlee BM, et al. Mycobacterial catalase-peroxidase is a tissue antigen and target of the adaptive immune response in systemic sarcoidosis. J Exp Med 2005;201(5):755-67.

21. Newman LS, Rose CS, Bresnitz EA, et al. A case control etiologic study of sarcoidosis: environmental and occupational risk factors. Am J Respir Crit Care Med 2004;170(12):1324-30.

22. Chen ES, Moller DR. Etiologies of sarcoidosis. Clin Rev Allergy Immunol 2015;49(1):6-18.

23. Hunninghake GW, Costabel U, Ando M, et al. ATS/ERS/WASOG statement on sarcoidosis. American Thoracic Society/European Respiratory Society/World Association of Sarcoidosis and other Granulomatous Disorders. Sarcoidosis Vasc Diffuse Lung Dis 1999;16(2):149-73.

24. Zissel G, Muller-Quernheim J. Cellular players in the immunopathogenesis of sarcoidosis. Clin Chest Med 2015;36(4):549-60.

25. Broos $\mathrm{CE}$, van Nimwegen $\mathrm{M}$, Hoogsteden $\mathrm{HC}$, et al. Granuloma formation in pulmonary sarcoidosis. Front Immunol 2013;4:437.

26. Baughman RP, Culver DA, Judson MA. A concise review of pulmonary sarcoidosis. Am J Respir Crit Care Med 2011;183(5):573-81. 
27. Ungprasert P, Carmona EM, Utz JP, et al. Epidemiology of sarcoidosis 1946-2013: a population-based study. Mayo Clin Proc 2016;91(2):183-8.

28. Fritz $D$, Voortman $M$, van de Beek $D$, et al. Many faces of neurosarcoidosis: from chronic meningitis to myelopathy. Curr Opin Pulm Med 2017;23(5):439-46.

29. Stern BJ, Royal W, 3rd, Gelfand JM, et al. Definition and consensus diagnostic criteria for neurosarcoidosis: from the neurosarcoidosis consortium consensus group. JAMA Neurol 2018;75(12): 1546-1553.

30. Drent M, Lower EE, De Vries J. Sarcoidosis-associated fatigue. Eur Respir J 2012;40(1):255-63.

31. Marcellis RG, Lenssen AF, Elfferich $M D$, et al. Exercise capacity, muscle strength and fatigue in sarcoidosis. Eur Respir J 2011;38(3):628-34.

32. Strookappe B, De Vries J, Elfferich M, et al. Predictors of fatigue in sarcoidosis: the value of exercise testing. Respir Med 2016;116:49-54.

33. De Vries J, Michielsen H, Van Heck GL, Drent $M$. Measuring fatigue in sarcoidosis: the Fatigue Assessment Scale (FAS). Br J Health Psychol 2004;9(Pt 3):279-91.

34. Hendriks C, Drent M, Elfferich M, De Vries J. The Fatigue Assessment Scale: quality and availability in sarcoidosis and other diseases. Curr Opin Pulm Med 2018;24(5):495-503.

35. Voortman M, Fritz D, Vogels OJM, et al. Small fiber neuropathy: a disabling and underrecognized syndrome. Curr Opin Pulm Med 2017;23(5):447-57.

36. Lauria G, Lombardi R. Small fiber neuropathy: is skin biopsy the holy grail? Curr Diab Rep 2012;12(4):384-92.

37. Themistocleous AC, Ramirez JD, Serra J, Bennett DL. The clinical approach to small fibre neuropathy and painful channelopathy. Pract Neurol 2014;14(6):368-79.

38. Hoitsma E, De Vries J, Drent M. The small fiber neuropathy screening list: construction and crossvalidation in sarcoidosis. Respir Med 2011;105(1):95-100.

39. Elfferich MD, Nelemans PJ, Ponds RW, et al. Everyday cognitive failure in sarcoidosis: the prevalence and the effect of anti-TNF-alpha treatment. Respiration 2010;80(3): 212-9.

40. Drent M, Hendriks C, Elfferich M, De Vries J. Sarcoidosis-associated disability. In: Sarcoidosis: a clinician's guide. Editors: RP Baughman and D. Valeyre. Publisher: Elsevier. ISBN: 987-0-323-54429-0. 2019; pgn 257-264.

41. Hendriks C, Drent M, De Kleijn W, et al. Everyday cognitive failure and depressive symptoms predict fatigue in sarcoidosis: a prospective follow-up study. Respir Med 2018;138S:S24-S30.

42. Bakkers M, Faber CG, Hoeijmakers JG, et al. Small fibers, large impact: quality of life in small-fiber neuropathy. Muscle Nerve 2014;49(3):329-36.

43. Drent M, Strookappe B, Hoitsma E, De Vries J. Consequences of sarcoidosis. Clin Chest Med 2015;36(4):727-37.

44. James WE, Baughman R. Treatment of sarcoidosis: grading the evidence. Expert Rev Clin Pharmacol 2018;11(7):677-87.

45. Bitoun S, Bouvry D, Borie R, et al. Treatment of neurosarcoidosis: a comparative study of methotrexate and mycophenolate mofetil. Neurology 2016;87(24):2517-21.

46. Gelfand JM, Bradshaw MJ, Stern BJ, et al. Infliximab for the treatment of CNS sarcoidosis: a multiinstitutional series. Neurology 2017;89(20):2092-100.

47. Hoitsma E, Faber CG, van Santen-Hoeufft M, et al. Improvement of small fiber neuropathy in a sarcoidosis patient after treatment with infliximab. Sarcoidosis Vasc Diffuse Lung Dis 2006;23(1):73-7.

48. Parambil JG, Tavee JO, Zhou L, et al. Efficacy of intravenous immunoglobulin for small fiber neuropathy associated with sarcoidosis. Respir Med 2011;105(1):101-5.

49. Wijnen PA, Cremers JP, Nelemans PJ, et al. Association of the TNF-alpha G-308A polymorphism with TNF-inhibitor response in sarcoidosis. Eur Respir J 2014;43(6):1730-9.

50. Tavee JO, Karwa K, Ahmed Z, et al. Sarcoidosis-associated small fiber neuropathy in a large cohort: clinical aspects and response to IVIG and anti-TNF alpha treatment. Respir Med 2017;126:135-8.

51. Dahan A, Dunne A, Swartjes M, et al. ARA 290 improves symptoms in patients with sarcoidosisassociated small nerve fiber loss and increases corneal nerve fiber density. Mol Med 2013;19:334-45.

52. Heij L, Niesters M, Swartjes M, et al. Safety and efficacy of ARA 290 in sarcoidosis patients with symptoms of small fiber neuropathy: a randomized, double-blind pilot study. Mol Med 2012;18: 1430-6. 
53. Culver DA, Dahan A, Bajorunas D, et al. Cibinetide improves corneal nerve fiber abundance in patients with sarcoidosis-associated small nerve fiber loss and neuropathic pain. Invest Ophthalmol Vis Sci 2017;58(6):BIO52-60.

54. Lower EE, Malhotra A, Surdulescu V, Baughman RP. Armodafinil for sarcoidosis-associated fatigue: a double-blind, placebo-controlled, crossover trial. J Pain Symptom Manage 2013;45(2):159-69.

55. Karadalli MN, Bosnak-Guclu M, Camcioglu B, et al. Effects of inspiratory muscle training in subjects with sarcoidosis: a randomized controlled clinical trial. Respir Care 2016;61(4):483-94.

56. Strookappe B, Swigris J, De Vries J, et al. Benefits of physical training in sarcoidosis. Lung 2015;193(5):701-8.

57. Marcellis R, Van der Veeke M, Mesters I, et al. Does physical training reduce fatigue in sarcoidosis? Sarcoidosis Vasc Diffuse Lung Dis 2015;32(1):53-62.

58. Strookappe B, Saketkoo LA, Elfferich M, et al. Physical activity and training in sarcoidosis: review and experience-based recommendations. Expert Rev Respir Med 2016;10(10):1057-68. 
Part 



\section{Chapter}

\section{Many faces of neurosarcoidosis: from chronic meningitis to myelopathy}

D. Fritz, M. Voortman, D. van de Beek, M. Drent, M.C. Brouwer Current Opinion of Pulmonology 2017;23(5):439-446 


\section{Abstract}

\section{Purpose of review}

Neurosarcoidosis occurs in $5 \%$ of patients with sarcoidosis and can be difficult to diagnose. In this review we discuss the most recent advances in our understanding of the disease, describing clinical characteristics, the diagnostic process, treatment and prognosis.

\section{Recent findings}

Clinical presentation is heterogeneous with most patients presenting with cranial nerve palsy, headache and sensory abnormalities. Patients are classified according to probability of the diagnosis according to the Zajicek criteria. In these criteria histopathological confirmation of non-caseating granulomas in affected tissue outside the nervous system is key. Radiological abnormalities on neuroimaging are non-specific. No biomarkers have been described that adequately identify patients with sarcoidosis. However, soluble interleukin-2 receptor is a relatively novel biomarker and may be useful. In addition to HRCT scan, ${ }^{18}$ F-FDG PET-CT scanning can identify occult locations of disease activity and aid in obtaining histological evidence of the disease. Despite the use of new therapies, still a third of the patients remain stable, deteriorate or die.

\section{Summary}

Diagnosing and treating patients with neurosarcoidosis remains a challenge. Long-term prospective studies evaluating patients suspected of neurosarcoidosis are needed to assess sensitivity and specificity of ancillary investigations and the diagnostic criteria. Furthermore, future studies are needed to evaluate the prognosis and the optimal treatment strategy. 


\section{Introduction}

Sarcoidosis is a multisystem disorder, caused by an exaggerated immune response to an unknown antigen in genetic predisposed individuals. ${ }^{1}$ It is characterized by granulomatous inflammation that can affect every organ system. Involvement of the nervous system is called neurosarcoidosis. Because of the heterogeneous clinical presentation and low sensitivity of ancillary investigations, the diagnosis of neurosarcoidosis can be difficult.

International literature concerning neurosarcoidosis predominantly consists of retrospective studies and no randomized controlled trials have been performed to assess treatment strategies..$^{2^{* *}}$ In this review we give an overview of the most recent advances in our understanding of the disease, clinical characteristics, the diagnostic process, treatment and prognosis.

\section{Epidemiology and risk factors}

Sarcoidosis is estimated to occur in 10-65 per 100000 persons in whom the nervous system is involved in approximately $5 \% .^{1,2^{* *}, 3}$ However, in autopsy studies granulomatous inflammation of the nervous system is seen in up to $25 \%$ of patients. ${ }^{4}$ Neurosarcoidosis affects women slightly more frequently than men (55\% vs. $45 \%$ ) as was reported in a recent meta-analysis including 1088 patients with neurosarcoidosis, with a mean age at presentation of 43 years. ${ }^{2 * *}$ The cause of sarcoidosis has not yet been elucidated. Studies show that sarcoidosis occurs in families, with an increased risk of first-degree and second-degree relatives. ${ }^{5}$ Several alleles in the human leukocyte antigen (HLA) region are associated with sarcoidosis, supporting the hypothesis that sarcoidosis manifests after exposure to so-far unidentified antigens. ${ }^{6}$ In addition, HLA typing of patients with sarcoidosis may identify patients with an increased risk for extra-pulmonary manifestations, but the clinical utility remains unknown. ${ }^{7}$ Only one study reported specific genetic risk factors for neurosarcoidosis. ${ }^{8}$ In this imputationbased fine-mapping study of the chromosomal region 15q25 in African-Americans with neurosarcoidosis, the investigators found ZNF592 to be highly associated in patients with neurosarcoidosis compared to healthy controls. However, when comparing neurosarcoidosis cases against sarcoidosis cases without neurological involvement, they found no association. 


\section{Clinical characteristics}

In $69 \%$ of patients with neurosarcoidosis, neurological involvement is the first presenting symptom. A neurological presentation without systemic involvement is seen in $52 \%$ of patients. . $^{* *}$ During the disease course, systemic involvement consists of pulmonary sarcoidosis in $67 \%$, ophthalmological sarcoidosis in $25 \%$ and skin and joint involvement both in $21 \% .^{{ }^{2 * *}}$ Sarcoidosis stays confined to the nervous system in $22 \%$ of the cases. ${ }^{2 * *}$ Most patients present with more than one neurological symptom and up to half develop additional neurological symptoms during the disease course..$^{9^{*}}$

The clinical presentation is heterogeneous and the three most frequently described presenting symptoms in a meta-analysis including 1088 patients were cranial nerve palsy (55\%), headache (32\%), and sensory abnormalities (29\%). Neurological symptoms consistent with spinal cord involvement were found in $18 \%$, peripheral neuropathy in $17 \%$, aseptic meningitis in $16 \%$, myopathy in $15 \%$, and neuro-endocrine involvement in $9 \%$.

\section{Cranial neuropathy}

Cranial neuropathy can be caused by direct granulomatous infiltration, increased intracranial pressure, or basilar aseptic meningitis. ${ }^{10}$ In one study evaluating cranial nerve involvement in 305 patients, $44 \%$ of patients had involvement of more than one cranial nerve. ${ }^{1{ }^{*}}$ Cranial nerve palsy mainly consisted of facial nerve (24\%) and optical nerve palsy (21\%). It tends to be unilateral, but especially optical, vestibulocochlear, facial, and trigeminal nerve palsy can manifest bilaterally. ${ }^{11^{*}}$

\section{Aseptic meningitis}

Neurosarcoidosis is an important cause of non-infectious meningitis and can manifest with sub-acute or chronic symptoms. ${ }^{10,12}$ Symptoms include headache, nuchal rigidity, or cranial nerve palsy. Hydrocephalus occurs in $9 \%$ of patients and is a life-threatening complication..$^{2^{* *}}$ It can be communicating or noncommunicating, resulting from either abnormal arachnoid villi absorption, subependymal inflammation, or granulomatous obstruction. $^{13-15}$

\section{Cerebral involvement and mass lesions}

A variety of cerebral lesions can be observed in patients with neurosarcoidosis, varying from dural mass lesions mimicking meningioma's to multiple or singular parenchymal nodular lesions. ${ }^{16,17}$ Symptoms include focal symptoms and seizures. Seizures are described in $14 \%$ of 965 patients. $^{2^{* *}}$ Hypothalamic-pituitary involvement was found in 
$9 \%$ of 1034 patients. $^{2^{* *}}$ In a multicenter study of 24 patients with neurosarcoidosis with pituitary involvement, the most common hormonal abnormalities were gonadotropin deficiency (88\%), TSH deficiency (63\%), and hyperprolactinemia (50\%). ${ }^{18}$

\section{Spinal cord involvement}

Common presenting symptoms of spinal cord sarcoidosis are paraparesis, paresthesia, and bowel, bladder, and sexual dysfunction. ${ }^{19,20^{*}}$ The majority of patients with spinal cord involvement present with insidious chronic symptoms for more than 2 months. ${ }^{20^{*}}$

\section{Peripheral neuropathy}

Peripheral nerve involvement in neurosarcoidosis is heterogeneous. It includes mononeuropathy, multiple mononeuropathy and sensory, sensorimotor, or motor polyneuropathies. $^{21,22}$ In addition, radiculopathy and polyradiculopathy have been described. ${ }^{23}$ It is hypothesized that peripheral neuropathy may occur because of granulomatous involvement of the nerve directly or in close proximity of blood vessels, ultimately leading to localized vasculitis and epineurium and/or perineurinum involvement. ${ }^{21}$ Small fiber neuropathy is rather common in sarcoidosis as well and is considered an epiphenomenon as there is no granulomatous inflammation of the small fibers. This syndrome is not specific for sarcoidosis and is observed in multiple disorders.

\section{Diagnosis}

Two sets of diagnostic criteria have been established: the Zajicek criteria and the WASOG criteria. ${ }^{17,24-26}$ The Zajicek criteria were established in 1999 and modified by Marangoni et al. and Tavee et al. (Table 2.1). The World Association of Sarcoidosis and Other Granulomatous Disorders (WASOG) updated their criteria in 2014 (Table 2.2). A certain (or 'definite') diagnosis can only be established by biopsy of affected nervous system tissue. Frequently, this is either not possible or considered too invasive as for instance stereotactic brain biopsy has been associated with morbidity in $3.5 \%$ and mortality in $0.7 \%$ of patients. ${ }^{17,27,28}$ Therefore, the diagnostic process is commonly steered towards performing biopsy outside the central nervous system. ${ }^{24,25}$

The essence of both sets of criteria is a clinical presentation suggestive for neurosarcoidosis, characteristic abnormalities in cerebrospinal fluid (CSF), or on MRI, evidence of noncaseating granulomas, and no evidence of alternative diagnoses. Sensitivity and specificity of both criteria are unknown. Recent published articles mostly 
use the Zajicek criteria $^{9 *, 17}$ The main disadvantage of the WASOG criteria is the exclusion of peripheral nerve involvement.

Table 2.1 Modified Zajicek criteria.

\begin{tabular}{ll}
\hline A clinical presentation suggestive of neurosarcoidosis in which all other diagnoses have been excluded \\
\hline definite neurosarcoidosis & positive nervous system histology \\
probable neurosarcoidosis & signs of inflammation in the central or peripheral nervous system on \\
& MRI or CSF (elevated protein, cells, immunoglobulin G indices, or the \\
& presence of oligoclonal bands) compatible with neurosarcoidosis; and \\
& positive histology for a systemic lesion or positive results for at least \\
& two of the following tests: ${ }^{18} \mathrm{~F}-\mathrm{FDG}$ PET, gallium scan, chest HRCT, \\
& serum ACE \\
possible neurosarcoidosis & above criteria are not met
\end{tabular}

$\mathrm{ACE}=$ angiotensin-converting enzyme; $\mathrm{CSF}=$ cerebrospinal fluid; $\mathrm{FDG}-\mathrm{PET}=$ fluordoexyglucose positron emission tomography; HRCT=high resolution computed tomography. Data from Tavee et al. and Zajicek et al. ${ }^{17,24}$

Table 2.2 WASOG criteria.

\begin{tabular}{|c|c|}
\hline $\begin{array}{l}\text { highly probable neurosarcoidosis: } \\
\text { at least } 90 \% \text { likelihood }\end{array}$ & $\begin{array}{l}\text { when clinical signs consistent with granulomatous inflammation of } \\
\text { the meninges, brain, ventricular (CSF) system, cranial nerves, pituitary } \\
\text { gland, spinal cord, cerebral vasculature or nerve routs alongside } \\
\text { imaging characteristics of disease (predominantly MRI gadolineum } \\
\text { enhancement) or a cerebrospinal fluid exam demonstrating } \\
\text { inflammation }\end{array}$ \\
\hline $\begin{array}{l}\text { probable neurosarcoidosis: } \\
\text { a } 50-89 \% \text { likelihood }\end{array}$ & $\begin{array}{l}\text { isolated facial palsy with negative MRI-brain or clinical syndrome } \\
\text { consistent with granulomatous inflammation of meninges, brain, } \\
\text { ventricular (CSF) system, cranial nerves, pituitary gland, spinal cord, } \\
\text { cerebral vasculature or nerve routs but without characteristic MRI or } \\
\text { CSF findings }\end{array}$ \\
\hline possible neurosar & seizure or cognitive decline with negative MRI. \\
\hline
\end{tabular}
less then $50 \%$ likelihood

$\mathrm{CSF}=$ cerebrospinal fluid. Biopsy of that organ demonstrating granulomatous inflammation of no alternate cause implies highly probable involvement; another organ has demonstrated granulomatous inflammation of no alternate cause; alternative causes for the clinical manifestation have been reasonable excluded. Based on Judson et al. ${ }^{25}$

\section{Ancillary investigations}

Various serological biomarkers, predominantly ACE and soluble interleukin-2 receptor (sIL2r), are associated with sarcoidosis, but none have been established to be sufficiently accurate to diagnose patients with neurosarcoidosis, although some might have a role in following disease activity. ${ }^{1}$ In the meta-analysis, serum ACE was elevated in only $35 \%$ of 674 patients neurosarcoidosis. ${ }^{2 * *}$ The value of genotype-corrected ACE has not been evaluated in neurosarcoidosis, but in systemic sarcoidosis it does not improve the diagnostic accuracy considerably. ${ }^{1,29}$ Serum sIL2r has been considered a 
promising biomarker in sarcoidosis. ${ }^{1}$ Serum sIL2r was more frequently elevated in patients with sarcoidosis and extra-pulmonary involvement compared to healthy controls and has been shown to predict ${ }^{18}$ F-FDG avidity. ${ }^{30,31}$ However, a study evaluating serum sIL2 $r$ in neurosarcoidosis showed no differences between patients with neurosarcoidosis and those with tuberculous, bacterial or viral meningitis, multiple sclerosis, cerebral vasculitis, and healthy controls. ${ }^{32}$ CSF examination can be useful to exclude infections and carcinomatous meningitis. Pleiocytosis was found in $58 \%$ of 730 , elevated CSF protein in $63 \%$ of 729 , and normal CSF in $27 \%$ of 332 patients. ${ }^{2 * *}$ CSF ACE is considered to have a too low sensitivity for the diagnosis of neurosarcoidosis and not to be associated with disease activity. ${ }^{17,33^{*}}$ Recently, a study was published evaluating the value of CSF sIL2r and showed that levels above $150 \mathrm{pg} / \mathrm{ml}$ identified patients with untreated neurosarcoidosis with a sensitivity of $63 \%$ and a specificity of $93 \%$ compared to non-infectious neurological diseases. ${ }^{32}$ However, in another study with patients with clinically isolated neurosarcoidosis CSF sIL2r was elevated in only $46 \%{ }^{34}$

Gadolineum enhanced MRI is the first choice imaging modality to assess central nervous system involvement of sarcoidosis and evaluate disease activity. ${ }^{17}$ Abnormalities suggestive for neurosarcoidosis includes leptomeningeal enhancement, tumour-like lesions, cranial nerve enhancement, and hydrocephalus (Figure 2.1). ${ }^{35^{*}}$ Spinal cord lesions are most frequently localized intramedullary in $81 \%$ of the cases and the majority extends over three or more spinal segments. ${ }^{19}$ In the meta-analysis, abnormalities suggestive for neurosarcoidosis on cranial MRI were seen in $78 \%$ of 362 patients and on spinal MRI in $48 \%$ of 185 patients. ${ }^{2^{* *}}$ 

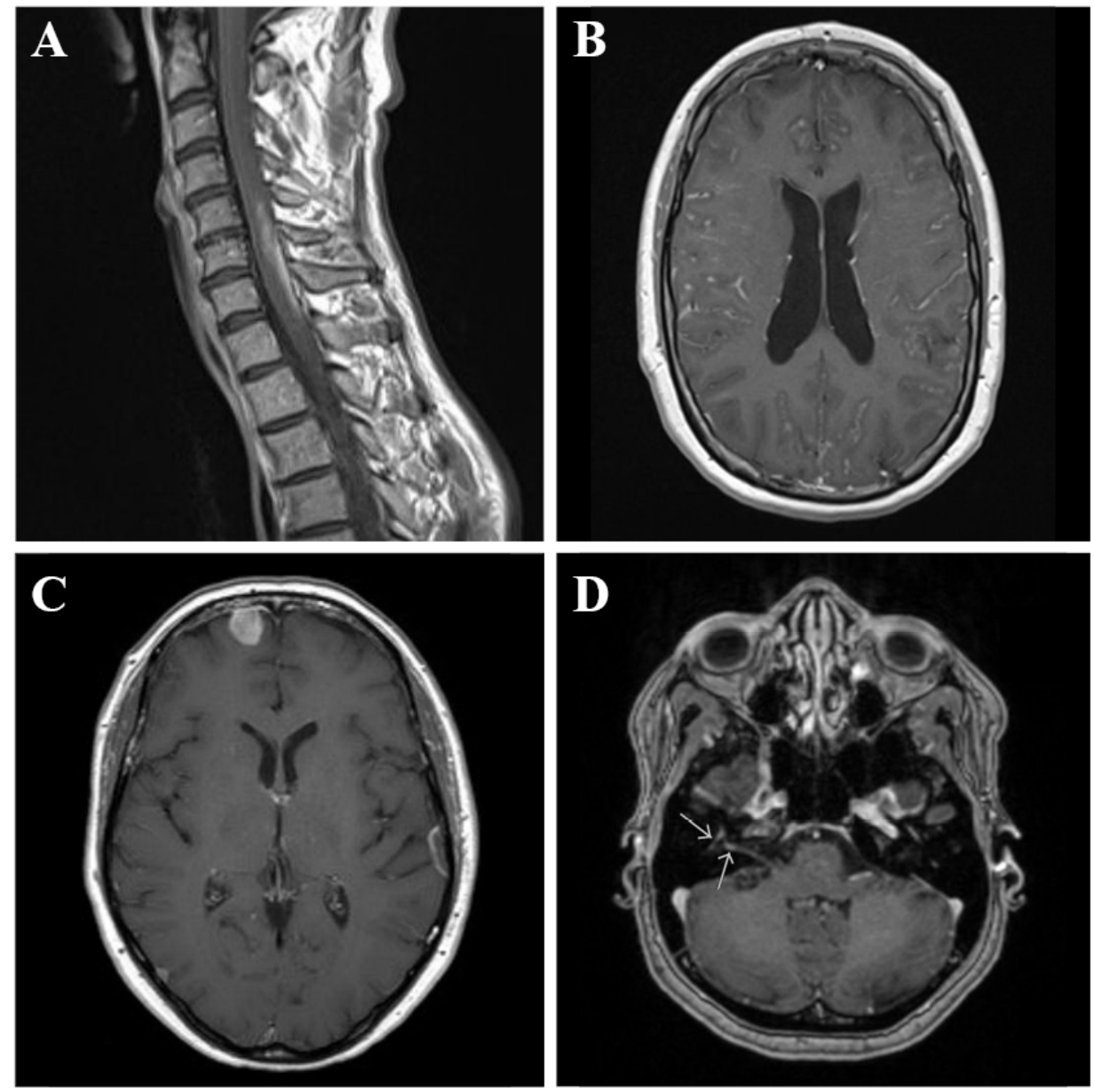

Figure 2.1 Abnormalities suggestive of neurosarcoidosis on neuroimaging. Abnormalities on magnetic resonance imaging (MRI) brain and spinal cord in patients with neurosarcoidosis consisting of gadolineum enhancement of a spinal cord lesion (A), leptomeningeal enhancement (B), gadolineum enhancement of dural lesions (C), and gadolineum enhancement of the right facial nerve (D).

In patients suspected of neurosarcoidosis and no prior history of sarcoidosis, an extensive systemic work-up should be performed to obtain a biopsy location outside the nervous system to achieve histopathological confirmation (Figure 2.2). ${ }^{17,36}$ The systemic work-up should include a high-resolution CT-scan (HRCT) and if negative or when biopsy is negative, a ${ }^{18} \mathrm{~F}$-FDG PET-CT should be considered. ${ }^{18} \mathrm{~F}$-FDG PET-CT can reveal occult sites of disease activity outside the central nervous system, even when serological markers are normal or when other imaging modalities are normal. ${ }^{37}$ In our 
tertiary referral center, we have evaluated the clinical utility of ${ }^{18}$ F-FDG PET-CT in patients suspected of neurosarcoidosis and 24 of 110 patients (22\%) showed abnormalities suggestive for sarcoidosis and this led to a biopsy confirmation in 12 of 20 patients (59\%; data not yet published). ${ }^{18}$ F-FDG PET-CT should preferably be performed before treatment with immunosuppressive therapy as this can lower the sensitivity. ${ }^{38,39}$
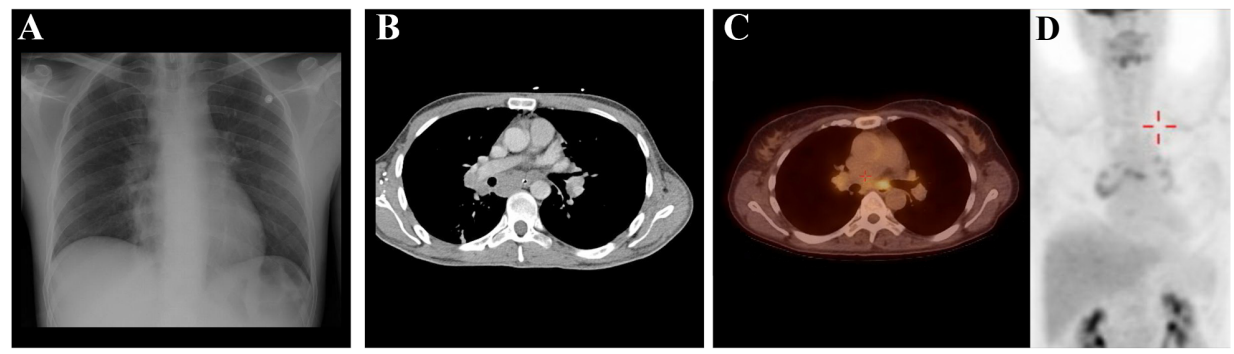

Figure 2.2 Abnormalities suggestive of neurosarcoidosis on systemic imaging. Abnormalities suggestive of sarcoidosis on systemic imaging consisting of hilar lymphadenopathy on chest X-ray (A), hilar and mediastinal lymphadenopathy on chest CT-scan (B), and hilar and mediastinal FDG activity on ${ }^{18}$ F-FDG PET-CT (C and D).

\section{Differential diagnosis and mimics}

The exclusion of other differential diagnoses is essential. Diagnoses to consider are tuberculosis, Behcet's disease, Sjögren's disease, granulomatosis with polyangiitis and eosinophilic granulomatosis with polyangiitis, central nervous system vasculitis, multiple sclerosis, neuromyelitis optica and malignancies, among others. ${ }^{17,36}$

As patients are being diagnosed according to probability and confidence of the diagnosis can be strengthened over time, they may initially be diagnosed with diseases mimicking neurosarcoidosis, which can present with similar histopathological findings. ${ }^{40}$ Examples include IgG4-related disease and a sarcoid-like reaction due to malignancies or medication use. IgG4-related disease can present with a chronic meningitis, cranial nerve palsy, or pituitary involvement. ${ }^{41}$ In 2012, consensus was reached that granulomatous inflammation excluded IgG4-related disease. ${ }^{42,43}$ However, recently two patients presenting with IgG4-related lymphadenopathy together with granulomas were described, underlining that in the appropriate clinical context granulomas should not exclude IgG4-related disease. ${ }^{44}$ Sarcoid-like reactions are described as a noncaseating granulomatous inflammation, without further evidence for sarcoidosis, in association with malignancies - most notably a lymphoma or malignancy of the breast 
- or certain medication. ${ }^{45}$ Tumour necrosis factor alpha (TNF- $\alpha$ ) antagonists can paradoxically cause a sarcoid-like reaction, as well as a variety of neurological symptoms, mimicking neurosarcoidosis. ${ }^{46-49}$

\section{Treatment}

Since neurosarcoidosis is a rare disease, no randomized, double-blind, placebocontrolled trials have been performed. Treatment options are based on expert opinion and are derived from evidence in the treatment of pulmonary sarcoidosis. ${ }^{50-52}$ Corticosteroids are the mainstay of treatment in neurosarcoidosis. In a meta-analysis, $83 \%$ of 655 patients were treated with corticosteroids during the course of the disease, although spontaneous remissions do occur and $15 \%$ of 655 patients did not receive any treatment. $^{2^{* *}}$ The optimal initial dose of corticosteroids in neurosarcoidosis varies and depends on disease severity with more aggressive therapy in patients with more severe disease. ${ }^{17,19,53}$ In patients with diabetes or obesitas, the dosage of prednisone should be low and tapered as soon as possible. In these patients second-line treatment should be considered early as steroid sparing agent. Prednisolone side-effects can be significant and are undesirable. ${ }^{54}$ Corticosteroid tapering is essential, even though symptoms may reoccur at lower dosages. ${ }^{24,55,56^{* *}}$ Reported treatment response to corticosteroids varies widely with progression of disease ranging from 24 to $71 \% .^{2^{* *}}$ Although the short-term effect of corticosteroids has been well established, there are limited data beyond 2 years to indicate whether oral steroids have a disease modifying effect on the longterm. ${ }^{57}$ Second-line treatment is recommended in case of severe disease at presentation, refractory, or relapsing disease during corticosteroid treatment, when prolonged treatment with corticosteroids is expected or a primary contra-indication for corticosteroids exists. ${ }^{51,53,58,59}$ Most second-line therapies (most importantly methotrexate and azathioprine) need 3-6 months before a clinical response might be expected. $^{52}$ In the meta-analysis, methotrexate was reported as the most frequently used second-line treatment ( $16 \%$ of 655 cases), followed by azathioprine in $8 \% .^{2^{* *}}$ Overall, second-line therapy was used in $27 \%$ of 539 patients..$^{2^{* *}}$ One study reported a response rate of $61 \%$ in patients treated with methotrexate and corticosteroids, after corticosteroids monotherapy failed. ${ }^{58}$ Moreover, the side effects of methotrexate are less severe compared to other second-line treatments. ${ }^{60}$ In pulmonary sarcoidosis, in addition to methotrexate, azathioprine is commonly used and is reported to have a similar treatment response and adverse events, except for a higher rate of infection compared to methotrexate. ${ }^{61}$ In a recent retrospective study comparing the efficacy of methotrexate and mycophenolate mofetil (MMF) in neurosarcoidosis, a higher relapse 
rate was observed in patients receiving MMF (11/14 [79\%]) compared to methotrexate (15/32 [47\%]). Furthermore, the authors reported a significantly shorter time to relapse in the MMF group (11 months MMF versus 28 months methotrexate treatment). ${ }^{5 *^{* *}}$

Third-line treatment consists of TNF- $\alpha$ antagonists and cyclophosphamide. In the metaanalysis, third-line treatment was started in $9 \%$ of 539 patients. ${ }^{2 * *}$ Increasingly, patients are being treated with TNF- $\alpha$ antagonists..$^{2^{* *}}$ A randomized controlled trial conducted in patients with chronic steroid-resistant pulmonary sarcoidosis showed a decrease in extra-pulmonary involvement after 24-weeks of treatment. ${ }^{62}$ In a retrospective study 18 patients with biopsy proven neurosarcoidosis were treated with infliximab, of whom 16 patients did not respond to at least one immunosuppressant drug in addition to corticosteroids (including 11 patients after cyclophosphamide treatment). At 6 months after initiation of infliximab treatment, 33\% had a complete remission, 56\% a partial remission, and stable disease was observed in $11 \% .{ }^{63^{* *}}$ Other case reports and case series also reported beneficial effects of TNF- $\alpha$ antagonists in refractory neurosarcoidosis, not responding to other agents. ${ }^{64-78}$ When treating patients with long-term infliximab, anti-infliximab antibodies formation can lead to treatment failure. ${ }^{79}$ Therefore, concomitant methotrexate treatment is strongly recommended as this has been shown to be efficient in reducing immunogenicity in a dose-dependent manner. ${ }^{80,81}$ Recently, responsiveness to TNF- $\alpha$ antagonists has been associated with the TNF- $\alpha$ G-308A polymorphism. ${ }^{82}$ Cyclophosphamide is recommended as a third-line treatment option in severe disease, refractory to other cytotoxic agents because of a similar reported treatment response and its high toxicity. ${ }^{58,83}$ There is widespread recognition that new therapeutic options should be developed for sarcoidosis. A better insight into the advantages and disadvantages of second-line and third-line treatment is important. The long-term effects of immunosuppressive agents, the optimal starting and maintenance dosages, and the best interval and discontinuation regimens should be elucidated. Identified associations of polymorphisms with treatment response suggest a step towards personalized medicine. ${ }^{82}$ Future research should focus on the role for pharmacogenetics and phenotypic predictors of treatment response and toxicity. ${ }^{84}$ Neurosurgical intervention may be needed for obstructive hydrocephalus. ${ }^{14,85}$ In our cohort study we described this treatment in 3 of 52 patients $(6 \%)$. $^{9^{*}}$ Furthermore, especially in extramedullary involvement spinal decompressive surgery can be indicated. ${ }^{86}$ Cranial mass lesions have been described to respond to radiation therapy, although this is not recommended as standard treatment. ${ }^{50}$ Symptomatic treatment consists of anti-epileptic medication and hormonal substitution among others. ${ }^{2^{* *}}$ 


\section{Prognosis}

In the meta-analysis outcome could be assessed in 415 patients and showed complete remission in $27 \%$, incomplete remission in $32 \%$, stable disease in $24 \%$, and deterioration in $6 \% .^{2^{* *}}$ Mortality was reported in 42 of 826 patients (5\%). Despite the increased usage of second-line and third-line therapy, still a third of the patients remain stable, deteriorate or die..$^{2^{* *}}$ Our experience suggests that neurosarcoidosis is a chronic disease with $71 \%$ of 52 patients having residual symptoms at the end of follow-up, leading to functional impairment in $29 \% .{ }^{{ }^{*}}$ Neurological involvement in conjunction with lung involvement is associated with an increased mortality (hazard ratio $=4.18 ; 95 \%$ confidence interval 1.44-12.1). ${ }^{3}$ Patients with neurosarcoidosis more often have a chronic disease course compared to other forms of sarcoidosis (odds ratio=19.24). ${ }^{87}$

\section{Conclusion}

Diagnosing and treating patients with neurosarcoidosis remains a challenge. Recent advances have mainly been made by combining all the available data to shed light on the prevalence of neurosarcoidosis and its manifestations. Currently, a national neurosarcoidosis registry in the Netherlands is being conducted to prospectively describe the various manifestations of neurosarcoidosis. Furthermore, recent studies have described the use of CSF SIL2 $r$ and ${ }^{18}$ F-FDG PET-CT, which may be useful in the diagnostic process. Long-term prospective studies evaluating patients suspected of neurosarcoidosis are needed to assess sensitivity and specificity of ancillary investigations and the diagnostic criteria. Furthermore, future studies are needed to evaluate the prognosis and the optimal treatment strategy. 


\section{References}

Papers of particular interest, published within the annual period of review, have been highlighted as:

* of special interest

** of outstanding interest

1. Valeyre D, Prasse A, Nunes H, et al. Sarcoidosis. Lancet 2014;383(9923):1155-67.

2. Fritz $D$, van de Beek D, Brouwer MC. Clinical features, treatment and outcome in neurosarcoidosis: systematic review and meta-analysis. BMC Neurol 2016;16(1):220.

** A meta-analysis combining all the available data published between 1980 and 2016, including 1088 patients as decribed in 29 articles. This meta-analysis sheds light on clinical characteristics, diagnostic modalities, treatment and outcome. Furthermore, it describes temporal trends in treatment strategies and outcome over time.

3. Ungprasert P, Carmona EM, Utz JP, et al. Epidemiology of sarcoidosis 1946-2013: a population-based study. Mayo Clin Proc 2016;91(2):183-8.

4. Iannuzzi MC, Rybicki BA, Teirstein AS. Sarcoidosis. N Engl J Med 2007;357(21):2153-65.

5. Rybicki BA, lannuzzi MC, Frederick MM, et al. Familial aggregation of sarcoidosis. A case-control etiologic study of sarcoidosis (ACCESS). Am J Respir Crit Care Med 2001;164(11):2085-91.

6. Fingerlin TE, Hamzeh N, Maier LA. Genetics of sarcoidosis. Clin Chest Med 2015;36(4):569-84.

7. Darlington $P$, Gabrielsen $A$, Sorensson $P$, et al. HLA-alleles associated with increased risk for extrapulmonary involvement in sarcoidosis. Tissue Antigens 2014;83(4):267-72.

8. Lareau CA, Adrianto I, Levin AM, et al. Fine mapping of chromosome 15q25 implicates ZNF592 in neurosarcoidosis patients. Ann Clin Transl Neurol 2015;2(10):972-7.

9. Leonhard SE, Fritz D, Eftimov F, et al. Neurosarcoidosis in a tertiary referral center: a cross-sectional cohort study. Medicine (Baltimore) 2016;95(14):e3277.

* In this retrospective observational study 52 patients diagnosed with neurosarcoidosis are described, focussing on clinical characteristics, diagnosis, treatment and outcome.

10. Zunt JR, Baldwin KJ. Chronic and subacute meningitis. Continuum (Minneap Minn) 2012;18(6 Infectious Disease):1290-318.

11. Carlson ML, White JR, Jr., Espahbodi M, et al. Cranial base manifestations of neurosarcoidosis: a review of 305 patients. Otol Neurotol 2015;36(1):156-66.

* In this retrospective study, 305 patients with cranial base manifestations have been described, foccusing on cranial nerve palsy. This is one of the largest retrospective cohorts of neurosarcoidosis patients.

12. Hahn LD, Fulbright R, Baehring JM. Hypertrophic pachymeningitis. J Neurol Sci 2016;367:278-83.

13. Yoshitomi M, Uchikado H, Hattori G, et al. Endoscopic biopsy for the diagnosis of neurosarcoidosis at the fourth ventricle outlet with hydrocephalus. Surg Neurol Int 2015;6(Suppl 25):S633-6.

14. Benzagmout M, Boujraf S, Gongora-Rivera F, et al. Neurosarcoidosis which manifested as acute hydrocephalus: diagnosis and treatment. Intern Med 2007;46(18):1601-4.

15. Tabuchi S, Uno T. Hydrocephalus with panventricular enlargement as the primary manifestation of neurosarcoidosis: a case report. J Med Case Rep 2013;7:240.

16. Rodriguez F, Link MJ, Driscoll CL, Giannini C. Neurosarcoidosis mimicking meningioma. Arch Neurol 2005;62(1):148-9.

17. Tavee JO, Stern BJ. Neurosarcoidosis. Continuum (Minneap Minn) 2014;20(3 Neurology of Systemic Disease):545-59.

** In this review article, the most recent update of the Zajicek criteria was proposed.

18. Langrand $\mathrm{C}$, Bihan $\mathrm{H}$, Raverot $\mathrm{G}$, et al. Hypothalamo-pituitary sarcoidosis: a multicenter study of 24 patients. QJM 2012;105(10):981-95.

19. Sohn M, Culver DA, Judson MA, et al. Spinal cord neurosarcoidosis. Am J Med Sci 2014;347(3):195-8.

20. Durel CA, Marignier R, Maucort-Boulch D, et al. Clinical features and prognostic factors of spinal cord sarcoidosis: a multicenter observational study of 20 BIOPSY-PROVEN patients. J Neurol 2016;263(5):981-90. 
* A retrospective cohort describing the clinical characteristics and prognostic factors of spinal cord sarcoidosis.

21. Said G, Lacroix C, Plante-Bordeneuve V, et al. Nerve granulomas and vasculitis in sarcoid peripheral neuropathy: a clinicopathological study of 11 patients. Brain 2002;125(Pt 2):264-75.

22. Scott TS, Brillman J, Gross JA. Sarcoidosis of the peripheral nervous system. Neurol Res 1993;15(6):38990.

23. Fahoum F, Drory VE, Issakov J, Neufeld MY. Neurosarcoidosis presenting as Guillain-Barre-like syndrome. A case report and review of the literature. J Clin Neuromuscul Dis 2009;11(1):35-43.

24. Zajicek JP, Scolding NJ, Foster $\mathrm{O}$, et al. Central nervous system sarcoidosis-diagnosis and management. QJM 1999;92(2):103-17.

25. Judson MA, Costabel $U$, Drent $M$, et al. The WASOG sarcoidosis organ assessment instrument: an update of a previous clinical tool. Sarcoidosis Vasc Diffuse Lung Dis 2014;31(1):19-27.

* In this delphi concensus study an update of the WASOG criteria was proposed.

26. Marangoni S, Argentiero V, Tavolato B. Neurosarcoidosis. Clinical description of 7 cases with a proposal for a new diagnostic strategy. J Neurol 2006;253(4):488-95.

27. Hall WA. The safety and efficacy of stereotactic biopsy for intracranial lesions. Cancer 1998;82(9):174955.

28. McGirt MJ, Woodworth GF, Coon AL, et al. Independent predictors of morbidity after image-guided stereotactic brain biopsy: a risk assessment of 270 cases. J Neurosurg 2005;102(5):897-901.

29. Biller H, Zissel G, Ruprecht B, et al. Genotype-corrected reference values for serum angiotensinconverting enzyme. Eur Respir J 2006;28(6):1085-90.

30. Mostard RL, Van Kuijk SM, Verschakelen JA, et al. A predictive tool for an effective use of (18)F-FDG PET in assessing activity of sarcoidosis. BMC Pulm Med 2012;12:57.

31. Gungor S, Ozseker F, Yalcinsoy M, et al. Conventional markers in determination of activity of sarcoidosis. Int Immunopharmacol 2015;25(1):174-9.

32. Petereit HF, Reske D, Tumani $H$, et al. Soluble CSF interleukin 2 receptor as indicator of neurosarcoidosis. J Neurol 2010;257(11):1855-63.

** In this study 139 paired CSF and serum samples from 11 patients with neurosarcoidosis, 21 with multiple sclerosis, 10 with central nervous system vasculitis, 22 with bacterial meningitis, 17 with viral meningitis/encefalitis, 7 with tuberculosis and 18 healthy doners were assessed for SIL2-R. They found that sIL2-R levens in CSF above $150 \mathrm{pg} / \mathrm{ml}$ identified NS patients a sensitivity of $63 \%$ and a specificity of 93\% compared to non-infectious neurological diseases.

33. Bridel C, Courvoisier DS, Vuilleumier N, Lalive PH. Cerebrospinal fluid angiotensin-converting enzyme for diagnosis of neurosarcoidosis. J Neuroimmunol 2015;285:1-3.

34. Wegener S, Linnebank $M$, Martin $R$, et al. Clinically isolated neurosarcoidosis: a recommended diagnostic path. Eur Neurol 2015;73(1-2):71-7.

35. Bathla G, Singh AK, Policeni B, et al. Imaging of neurosarcoidosis: common, uncommon, and rare. Clin Radiol 2016;71(1):96-106.

*A review describing the imaging spectrum of neurosarcoidosis, including follow-up imaging.

36. Hoitsma E, Drent M, Sharma OP. A pragmatic approach to diagnosing and treating neurosarcoidosis in the 21st century. Curr Opin Pulm Med 2010;16(5):472-9.

37. Teirstein AS, Machac J, Almeida O, et al. Results of 188 whole-body fluorodeoxyglucose positron emission tomography scans in 137 patients with sarcoidosis. Chest 2007;132(6):1949-53.

38. Mostard RL, van Kroonenburgh MJ, Drent M. The role of the PET scan in the management of sarcoidosis. Curr Opin Pulm Med 2013;19(5):538-44.

39. Keijsers RG, Verzijlbergen JF, van Diepen DM, et al. 18F-FDG PET in sarcoidosis: an observational study in 12 patients treated with infliximab. Sarcoidosis Vasc Diffuse Lung Dis 2008;25(2):143-9.

40. James DG. A clinicopathological classification of granulomatous disorders. Postgrad Med J 2000;76(898):457-65.

41. Stone JH, Zen Y, Deshpande V. IgG4-related disease. N Engl J Med 2012;366(6):539-51.

42. Deshpande V, Zen Y, Chan JK, et al. Consensus statement on the pathology of IgG4-related disease. Mod Pathol 2012;25(9):1181-92. 
43. Khosroshahi A, Wallace ZS, Crowe JL, et al. International consensus guidance statement on the management and treatment of IgG4-related disease. Arthritis Rheumatol 2015;67(7):1688-99.

44. Bateman AC, Ashton-Key MR, Jogai S. Lymph node granulomas in immunoglobulin G4-related disease. Histopathology 2015;67(4):557-61.

45. Ravaglia C, Gurioli C, Casoni GL, et al. Sarcoid-like lesion is a frequent benign cause of lymphadenopathy in neoplastic patients. Eur Respir J 2013;41(3):754-5.

46. Tong D, Manolios N, Howe G, Spencer D. New onset sarcoid-like granulomatosis developing during antiTNF therapy: an under-recognised complication. Intern Med J 2012;42(1):89-94.

47. Booker MJ, Flint J, Saravana S. Aseptic meningitis in a patient taking etanercept for rheumatoid arthritis: a case report. Cases J 2008;1(1):364.

48. Berrios I, Jun-O'Connell A, Ghiran S, lonete C. A case of neurosarcoidosis secondary to treatment of etanercept and review of the literature. BMJ Case Rep 2015;2015.

49. Hunter JB, Rivas A. Multiple cranial neuropathies following etanercept administration. Am J Otolaryngol 2016;37(3):259-62.

50. Nozaki K, Judson MA. Neurosarcoidosis: clinical manifestations, diagnosis and treatment. Presse Med 2012;41(6 Pt 2):e331-48.

51. Saketkoo LA, Baughman RP. Biologic therapies in the treatment of sarcoidosis. Expert Rev Clin Immunol 2016;12(8):817-25.

52. Baughman RP, Grutters JC. New treatment strategies for pulmonary sarcoidosis: antimetabolites, biological drugs, and other treatment approaches. Lancet Respir Med 2015;3(10):813-22.

53. Scott TF, Yandora K, Valeri A, et al. Aggressive therapy for neurosarcoidosis: long-term follow-up of 48 treated patients. Arch Neurol 2007;64(5):691-6.

54. Hoes JN, Jacobs JW, Verstappen SM, et al. Adverse events of low- to medium-dose oral glucocorticoids in inflammatory diseases: a meta-analysis. Annals of the rheumatic diseases 2009;68(12):1833-8.

55. Sharma OP. Pulmonary sarcoidosis and corticosteroids. The American review of respiratory disease 1993;147(6 Pt 1):1598-600.

56. Bitoun S, Bouvry D, Borie R, et al. Treatment of neurosarcoidosis: a comparative study of methotrexate and mycophenolate mofetil. Neurology 2016;87(24):2517-21.

** This is a retrospective multicenter comparative study describing 40 patients with a diagnosis of neurosarcoidosis based on a definite or probable diagnosis according to the Zajicek criteria receiving at least 3 months of either MTX (26 patients) and MMF ( 8 patients). MTX significantly increased survival time without relapse compared to MMF.

57. Paramothayan NS, Lasserson TJ, Jones PW. Corticosteroids for pulmonary sarcoidosis. Cochrane Database Syst Rev 2005(2):CD001114.

58. Lower EE, Broderick JP, Brott TG, Baughman RP. Diagnosis and management of neurological sarcoidosis. Arch Intern Med 1997;157(16):1864-8.

59. Agbogu BN, Stern BJ, Sewell C, Yang G. Therapeutic considerations in patients with refractory neurosarcoidosis. Arch Neurol 1995;52(9):875-9.

60. Cremers JP, Drent M, Baughman RP, et al. Therapeutic approach of hepatic sarcoidosis. Curr Opin Pulm Med 2012;18(5):472-82.

61. Vorselaars AD, Wuyts WA, Vorselaars VM, et al. Methotrexate vs azathioprine in second-line therapy of sarcoidosis. Chest 2013;144(3):805-12.

62. Judson MA, Baughman RP, Costabel U, et al. Efficacy of infliximab in extrapulmonary sarcoidosis: results from a randomised trial. Eur Respir J 2008;31(6):1189-96.

63. Cohen Aubart F, Bouvry D, Galanaud D, et al. Long-term outcomes of refractory neurosarcoidosis treated with infliximab. J Neurol 2017; 264(5):891-7.

** A retrospective cohort study describing the long-term outcomes of treatment with infliximab in biopsy proven neurosarcoidosis patients. They included 18 patients with biopsy proven neurosarcoidosis of whom 16 patients did not respond to at least 1 immunosuppressant drug in addition to corticosteroids (including 11 patients after cyclophosphamide treatment). At 6 months after initiation of infliximab treatment, $33 \%$ had a complete remission, $56 \%$ a partial remission and stable disease was observed in $11 \%$. After a median follow-up of 20 months $50 \%$ of patients had a relaps. Furthermore in 8 patients side effects were observed, 7 of which were infections. 
64. Pettersen JA, Zochodne DW, Bell RB, et al. Refractory neurosarcoidosis responding to infliximab. Neurology 2002;59(10):1660-1.

65. Carter JD, Valeriano J, Vasey FB, Bognar B. Refractory neurosarcoidosis: a dramatic response to infliximab. Am J Med 2004;117(4):277-9.

66. Kobylecki C, Shaunak S. Refractory neurosarcoidosis responsive to infliximab. Pract Neurol 2007;7(2):112-5.

67. Moravan M, Segal BM. Treatment of CNS sarcoidosis with infliximab and mycophenolate mofetil. Neurology 2009;72(4):337-40.

68. Sodhi M, Pearson K, White ES, Culver DA. Infliximab therapy rescues cyclophosphamide failure in severe central nervous system sarcoidosis. Respir Med 2009;103(2):268-73.

69. Chintamaneni S, Patel AM, Pegram SB, et al. Dramatic response to infliximab in refractory neurosarcoidosis. Ann Indian Acad Neurol 2010;13(3):207-10.

70. Santos E, Shaunak S, Renowden S, Scolding NJ. Treatment of refractory neurosarcoidosis with Infliximab. J Neurol Neurosurg Psychiatry 2010;81(3):241-6.

71. Riancho-Zarrabeitia L, Delgado-Alvarado M, Riancho J, et al. Anti-TNF-alpha therapy in the management of severe neurosarcoidosis: a report of five cases from a single centre and literature review. Clin Exp Rheumatol 2014;32(2):275-84.

72. Lorentzen AO, Sveberg L, Midtvedt $O$, et al. Overnight response to infliximab in neurosarcoidosis: a case report and review of infliximab treatment practice. Clin Neuropharmacol 2014;37(5):142-8.

73. Srivastava S, Rajakariar R, Ashman N, et al. Infliximab as long-term maintenance in steroid-resistant and recurrent sarcoidosis in a renal transplant with central nervous system involvement. Clin Kidney J 2012;5(1):53-5.

74. Lahmer T, Knopf A, Lanzl I, et al. Using TNF-alpha antagonist adalimumab for treatment for multisystem sarcoidosis: a case study. Rheumatology International 2012;32(8):2367-70.

75. Pereira J, Anderson NE, McAuley D, et al. Medically refractory neurosarcoidosis treated with infliximab. Intern Med J 2011;41(4):354-7.

76. Bomprezzi R, Pati S, Chansakul C, Vollmer T. A case of neurosarcoidosis successfully treated with rituximab. Neurology 2010;75(6):568-70.

77. Toth C, Martin L, Morrish W, et al. Dramatic MRI improvement with refractory neurosarcoidosis treated with infliximab. Acta Neurol Scand 2007;116(4):259-62.

78. Sollberger M, Fluri F, Baumann T, et al. Successful treatment of steroid-refractory neurosarcoidosis with infliximab. J Neurol 2004;251(6):760-1.

79. Baert $F$, Noman $M$, Vermeire $S$, et al. Influence of immunogenicity on the long-term efficacy of infliximab in Crohn's disease. N Engl J Med 2003;348(7):601-8.

80. Garces S, Demengeot J, Benito-Garcia E. The immunogenicity of anti-TNF therapy in immune-mediated inflammatory diseases: a systematic review of the literature with a meta-analysis. Annals of the rheumatic diseases 2013;72(12):1947-55.

81. Drent $\mathrm{M}$, Cremers JP, Jansen TL, Baughman RP. Practical eminence and experience-based recommendations for use of TNF-alpha inhibitors in sarcoidosis. Sarcoidosis Vasc Diffuse Lung Dis 2014;31(2):91-107.

82. Wijnen PA, Cremers JP, Nelemans PJ, et al. Association of the TNF-alpha G-308A polymorphism with TNF-inhibitor response in sarcoidosis. Eur Respir J 2014;43(6):1730-9.

83. Doty JD, Mazur JE, Judson MA. Treatment of corticosteroid-resistant neurosarcoidosis with a shortcourse cyclophosphamide regimen. Chest 2003;124(5):2023-6.

84. Vorselaars AD, Cremers JP, Grutters JC, Drent M. Cytotoxic agents in sarcoidosis: which one should we choose? Curr Opin Pulm Med 2014;20(5):479-87.

85. Scott TF, Brillman J. Shunt-responsive dementia in sarcoid meningitis: role of magnetic resonance imaging and cisternography. J Neuroimaging 2000;10(3):185-6.

86. Schaller B, Kruschat T, Schmidt H, et al. Intradural, extramedullary spinal sarcoidosis: report of a rare case and review of the literature. Spine J 2006;6(2):204-10.

87. Baughman RP, Lower EE. Features of sarcoidosis associated with chronic disease. Sarcoidosis Vasc Diffuse Lung Dis 2015;31(4):275-81. 


\section{Chapter 3}

\section{Clinical manifestations of neurosarcoidosis in the Netherlands}

M. Voortman, D. Fritz, O. J.M. Vogels, D. Van De Beek, J. De Vries, M. C. Brouwer, M. Drent J Neurol Neurosci 2019;10(2):292 


\section{Abstract}

\section{Background}

Neurosarcoidosis is a rare disease. We investigated the prevalence of different manifestations of neurosarcoidosis and the influence of race or gender in the Netherlands, as well as the treatment strategies for neurosarcoidosis patients.

\section{Methods}

Multicentre observational cohort study of patients with neurosarcoidosis in two tertiary referral centres; the ILD Center of Excellence of the St. Antonius Hospital, Nieuwegein and the Amsterdam University Medical Centre (UMC), the Netherlands, from 2015 till 2017, as well as participants of the Dutch Neurosarcoidosis Registry between June 2014 and December 2017.

\section{Results}

A total of 194 patients were included, 27 of whom were in the Dutch Neurosarcoidosis Registry, 70 treated at the ILD Center of Excellence and 97 treated at the Amsterdam UMC. Of the 194 patients included, 54\% were female. The most common neurosarcoidosis manifestations were chronic meningitis (41\%), cranial nerve dysfunction (39\%), myelopathy (28\%) and cerebral parenchymal disease (26\%). Age, gender and race did not differ for the various manifestations, except for peripheral polyneuropathy, which was more prevalent in men than in women ( $21 \%$ versus $10 \%)$. Most neurosarcoidosis patients were treated with prednisone $(89 \%)$, methotrexate (46\%) or infliximab (21\%). A total of 102 patients (53\%) received second- or third-line treatment.

\section{Conclusion}

In a large cohort of neurosarcoidosis patients, chronic meningitis, cranial nerve dysfunction, spinal cord involvement and cerebral parenchymal disease were the most common manifestations. Age, race and gender did not influence the clinical presentation, except for peripheral polyneuropathy (men>women). Over half of the patients required multiple immunosuppressive treatments. 


\section{Introduction}

Sarcoidosis is a multisystem inflammatory disorder of unknown aetiology in genetically predisposed individuals. ${ }^{1-3}$ The hallmark of sarcoidosis is granulomatous inflammation in the organs involved. ${ }^{4}$ The natural history and prognosis of sarcoidosis are highly variable and its course is often unpredictable. ${ }^{5-7}$ Sarcoidosis most often affects the lungs and lymph nodes, though any other organ system can be involved. ${ }^{8}$

Involvement of the nervous system, or neurosarcoidosis, is detected in $5 \%$ of cases during life, ${ }^{9-12}$ although autopsy studies have shown neurologic involvement in $25-50 \% .{ }^{13,14}$ Currently, the classification by Marangoni et al., ${ }^{15}$ including definite, probable and possible neurosarcoidosis is used. Neurosarcoidosis can involve any part of the nervous system, causing a variety of neurological symptoms. A meta-analysis showed that the most common manifestations of neurosarcoidosis were cranial nerve dysfunctions and myelopathy. ${ }^{16,17}$ Since neurologic involvement is the first presenting symptom in $50-70 \%$ of the neurosarcoidosis patients, and $52 \%$ of the patients have isolated neurosarcoidosis without systemic involvement, ${ }^{18-22}$ it can be difficult to diagnose. This might explain why previous studies reported variable prevalence of different manifestations. ${ }^{3,19,23-26}$

The influence of race on the epidemiology of sarcoidosis is generally recognized. The incidence of sarcoidosis in patients of African descent is high compared to that in Caucasians. ${ }^{27}$ Moreover, they often present with more severe organ involvement, a worse prognosis and higher mortality rates than Caucasians. ${ }^{27-29}$ It has also been acknowledged that the prevalence of various organ manifestations differs between different ethic subgroups, e.g. cutaneous and ocular sarcoidosis is more common in patients of African descent than in Caucasians, while hypercalcemia is more common in Caucasians. ${ }^{10}$ In terms of gender differences, pulmonary and cardiac sarcoidosis is more common in men, whereas more peripheral lymph node involvement, cutaneous, ocular and liver sarcoidosis are seen more often in women. ${ }^{10,30}$ To the best of our knowledge, racial and gender differences have so far not been studied specifically in neurosarcoidosis.

The aim of this study was to assess the prevalence of different manifestations of neurosarcoidosis and examine whether they vary with race and/or gender. In addition, the treatment strategies used for neurosarcoidosis patients were studied. 


\section{Methods}

\section{Study design}

In this multicentre study, all neurosarcoidosis patients evaluated and/or treated between 2015 and 2018 were included. Furthermore, patients participating in the Dutch Neurosarcoidosis Registry between June 2014 and 2018 were included. All patients with neurosarcoidosis in the Netherlands had been invited to join the Dutch Neurosarcoidosis Registry, an online registration study. ${ }^{31}$ Curavista (a certified eHealth platform) provided the online platform for this study and maintained the website. The present study was performed in accordance with the Declaration of Helsinki and its amendments. The patient registry and study protocol have been approved by the Medical Ethical Committee of the St. Antonius Hospital Nieuwegein. Digital or written informed consent was obtained from all patients.

\section{Study sample}

The overall study sample was comprised of patients from the ILD Center of Excellence of the St. Antonius Hospital Nieuwegein, and the Amsterdam University Medical Centre (UMC), the Netherlands, and the online Dutch Neurosarcoidosis Registry. We carefully checked for overlap in registrations between the different sources.

At the ILD Center of Excellence and the Amsterdam UMC, patients were identified by their treating physician and data were extracted from medical records, de-identified and collected in a database. Patients in the Dutch Neurosarcoidosis Registry had either been identified and entered by their physician with their consent or joined the online registry of their own accord. Data in the registry were self-reported by patients. Additional medical data were requested from their treating physician(s) and data were checked to see if the diagnosis of neurosarcoidosis had been confirmed. Curavista (a certified eHealth platform) collected the data online and exported de-identified data to a database. All patients participating in the Dutch Neurosarcoidosis Registry had sufficient command of Dutch and had access to the internet.

Multiple diagnostic criteria have been proposed for neurosarcoidosis. In this population, neurosarcoidosis was diagnosed according to the classification system by Zajicek, later modified by Marangoni, which labels patients as definite, probable or possible neurosarcoidosis. ${ }^{15,22}$ Definite neurosarcoidosis was diagnosed in case of a clinical presentation suggestive of neurosarcoidosis with exclusion of other possible diagnoses and the presence of positive nervous system histology. Probable neurosarcoidosis was diagnosed in case of a clinical presentation suggestive of neurosarcoidosis with MRI, CSF, and/or EMG/NCS findings typical of granulomatous 
inflammation of the nervous system and after rigorous exclusion of other causes and evidence for systemic sarcoidosis. Possible neurosarcoidosis was diagnosed in case of a clinical presentation suggestive of neurosarcoidosis with exclusion of alternative diagnosis where the above criteria are not met.

Patients with solely small fiber neuropathy (SFN) without granuloma formation in the affected tissue due to sarcoidosis were not considered to have neurosarcoidosis, ${ }^{32}$ and were excluded from this study ( $n=50$, Dutch neurosarcoidosis registry). Myopathy was also excluded from this analysis.

\section{Data collection and definition of phenotypes}

Demographic and clinical data were extracted from medical records. Onset of sarcoidosis and neurosarcoidosis and their various manifestations were also recorded. In order to determine associations between the various manifestations (systemic and neurosarcoidosis manifestations), we categorized neurosarcoidosis manifestations into 3 subgroups depending on anatomic location and severity; group 1: peripheral polyneuropathy; group 2: spinal cord manifestations (myelitis and polyradiculitis); and group 3: cerebral parenchymal lesions, neuro-endocrine, and brainstem involvement, cerebral infarction, chronic aseptic meningitis, hydrocephalus and cranial nerve manifestations.

\section{Statistical analysis}

All statistical analyses were performed using SPSS version 24. Data are presented as the means and range for continuous variables and as the number and percentage for categorical variables. In the analysis concerning race, we only compared Caucasians and patients of African descent, since other races were underrepresented and groups were therefore too small to analyse.

Differences between genders and races were analysed using the Chi-square test for dichotomous variables and the Mann-Whitney-U test for continuous variables. Differences between the various neurosarcoidosis groups were analysed using the Chisquare test for dichotomous variables and Kruskal-Wallis tests for continuous variables. A p-value of $<0.05$ was considered statistically significant.

\section{Results}

From the data sources 194 patients with neurosarcoidosis were identified: 27 participated in the Dutch Neurosarcoidosis Registry, 70 were recruited by the ILD 
Center of Excellence and 97 patients by the Amsterdam UMC. Demographic data are summarized in Table 3.1. Twelve patients (6\%) were classified as definite neurosarcoidosis, 153 patients (79\%) as probable neurosarcoidosis and 29 patients $(15 \%)$ as possible neurosarcoidosis. Ten patients (5\%) had an isolated neurosarcoidosis without systemic manifestations.

Table 3.1 Summary of the overall characteristics of the Dutch neurosarcoidosis sample studied, subdivided by gender.

\begin{tabular}{lcccc}
\hline Characteristic & $\begin{array}{c}\text { Female } \\
(\mathrm{n}=104)\end{array}$ & $\begin{array}{c}\text { Male } \\
(\mathrm{n}=90)\end{array}$ & $\begin{array}{c}\text { Total sample } \\
(\mathrm{n}=194)\end{array}$ & p-value* \\
\hline age at diagnosis of sarcoidosis (mean; range) & $44.5(13-80)$ & $43.1(20-79)$ & $43.8(13-80)$ & 0.443 \\
age at diagnosis of neurosarcoidosis (mean; range) & $45.9(15-80)$ & $46.1(20-79)$ & $46(15-80)$ & 0.939 \\
race & & & & 0.846 \\
Caucasian & $72(69.2)$ & $58(64.4)$ & $130(67)$ & \\
African descent & $22(21.2)$ & $19(21.1)$ & $41(21.1)$ & 0.277 \\
diagnostic classification of neurosarcoidosis & & & & \\
definite & $9(8.7)$ & $3(3.3)$ & $12(6.2)$ & \\
probable & $81(77.9)$ & $72(80)$ & $153(78.9)$ & \\
possible & $14(13.5)$ & $15(16.7)$ & $29(14.9)$ & \\
neurosarcoidosis manifestations & & & & \\
cranial nerve dysfunction & $46(44.2)$ & $30(33.3)$ & $76(39.2)$ & 0.121 \\
NI/NII/NIII/NIV/NV/NVI & $2 / 12 / 1 / 0 / 9 / 3$ & $0 / 7 / 4 / 1 / 7 / 5$ & $2 / 19 / 5 / 1 / 16 / 8$ & \\
NVII/NVIII/NIX/NX/NXI/NXII & $27 / 7 / 4 / 1 / 0 / 0$ & $14 / 9 / 1 / 1 / 0 / 1$ & $41 / 16 / 5 / 2 / 0 / 1$ & \\
chronic aseptic meningitis & $43(41.3)$ & $38(42.2)$ & $80(41.2)$ & 0.894 \\
spinal cord disease / myelitis & $27(26)$ & $28(31.1)$ & $55(28.4)$ & 0.427 \\
polyradiculitis & $1(0.5)$ & $0(0)$ & $1(0.5)$ & 0.351 \\
cerebral parenchymal disease & $31(29.8)$ & $19(21.1)$ & $50(25.8)$ & 0.168 \\
neuro-endocrine & $13(12.5)$ & $8(8.9)$ & $21(10.8)$ & 0.421 \\
brain stem & $6(5.8)$ & $5(5.6)$ & $11(5.7)$ & 0.951 \\
hydrocephalus & $12(11.5)$ & $8(8.9)$ & $20(10.3)$ & 0.547 \\
cerebral infarction & $5(4.8)$ & $9(10)$ & $14(7.2)$ & 0.162 \\
peripheral polyneuropathy & $10(9.6)$ & $19(21.1)$ & $29(14.9)$ & 0.025 \\
\hline
\end{tabular}

*p-value for difference between men and women; "Diagnostic classification according to Zajicek, as modified by Marangoni ${ }^{15,22}$

The most common manifestations of neurosarcoidosis were chronic meningitis (41\%), cranial nerve dysfunction (39\%), spinal cord involvement (28\%) and cerebral parenchymal disease (26\%; Table 3.1). Cerebral parenchymal disease varied from white matter lesions $(48 \%)$ to intracerebral lesions with mass effect $(30 \%)$ and intracerebral nodules (24\%). Multiple neurosarcoidosis manifestations occurred simultaneously in 109 patients (56\%).

Treatment for neurosarcoidosis had been initiated in 181 patients (93\%), of whom 173 patients (89\%) with prednisone. Second-line treatment had been given to 121 patients (62\%), 89 of whom had been treated with methotrexate (46\%), 24 patients with azathioprine $(12 \%)$, five patients with mycophenolate mofetil $(3 \%)$, two patients 
with cyclophosphamide (1\%) and one patient with cyclosporine (0.5\%). Third-line treatment had been started in 44 patients $(23 \%), 40$ of whom had been treated with infliximab (21\%) and four with adalimumab (2\%). Of the 13 patients (7\%) for whom no treatment had been initiated, six had shown spontaneous regression, while one had stable disease for years, three had no symptoms and three refused treatments. Patients with a facial nerve palsy as the only manifestation of neurosarcoidosis had mostly received prednisone (6 out of 7 cases). In 11 out of 20 cases with a hydrocephalus neurosurgical intervention was necessary (55\%).

A mortality rate of $2.1 \%$ was found in this cohort (four patients). The cause of death was neurosarcoidosis in two patients (hydrocephalus and cerebral lesions, respectively), infection due to immunosuppression in one patient and unknown cause in one patient.

No gender differences were found for the various neurosarcoidosis manifestations, except for peripheral polyneuropathy, which appeared to be more prevalent in men than in women $(21.1 \%$ versus $9.6 \%, p=0.025$; Table 3.1$)$. We found no significant differences between the two races regarding the various neurosarcoidosis manifestations (Table 3.2).

Table 3.2 Various neurosarcoidosis manifestations subdivided by race.

\begin{tabular}{lccc}
\hline Neurosarcoidosis manifestation & $\begin{array}{c}\text { Caucasian } \\
(\mathrm{n}=130)\end{array}$ & $\begin{array}{c}\text { African descent } \\
(\mathrm{n}=41)\end{array}$ & p-value* \\
\hline cranial nerve dysfunction & $51(39.2)$ & $18(43.9)$ & 0.595 \\
$\mathrm{NI} / \mathrm{NII} / \mathrm{NIII} / \mathrm{NIV/NV/NVI}$ & $2 / 13 / 4 / 1 / 8 / 6$ & $0 / 4 / 1 / 0 / 3 / 1$ & \\
$\quad \mathrm{NVII} / \mathrm{NVIII/NIX/NX/NXI/NXII}$ & $26 / 13 / 3 / 2 / 0 / 1$ & $9 / 1 / 1 / 0 / 0 / 0$ & \\
chronic aseptic meningitis & $50(38.4)$ & $20(48.8)$ & 0.241 \\
spinal cord disease / myelitis & $39(30)$ & $10(24.4)$ & 0.489 \\
cerebral parenchymal disease & $35(26.9)$ & $11(26.8)$ & 0.991 \\
neuro-endocrine & $12(9.2)$ & $5(12.2)$ & 0.580 \\
brain stem & $7(5.4)$ & $2(4.9)$ & 0.899 \\
hydrocephalus & $14(10.8)$ & $5(12.2)$ & 0.800 \\
cerebral infarction & $9(6.9)$ & $4(9.8)$ & 0.551 \\
peripheral neuropathy & $21(16.2)$ & $6(14.6)$ & 0.816 \\
\hline
\end{tabular}

${ }^{*} p$-value for difference between neurosarcoidosis patients of Caucasian and African descent.

\section{Various categories of neurosarcoidosis}

The total neurosarcoidosis sample was subdivided according to the various manifestations. Group 1 (peripheral polyneuropathy) comprised of 29 patients, 10 women and 19 men $(p=0.025)$. Group 2 (spinal cord manifestations [myelitis and polyradiculitis]) comprised of 56 patients, 28 women and 28 men (not significant). 
Group 3 (cerebral parenchymal lesions, neuro-endocrine, cerebral infarction and brainstem involvement, chronic aseptic meningitis, hydrocephalus and cranial nerve manifestations) comprised of 155 patients, 90 women and 65 men ( $p=0.012)$. No significant differences between these groups were found regarding age at time of diagnosis of sarcoidosis. Age at time of diagnosis of neurosarcoidosis was significantly different ( $p=0.001$ ); patients in group 3 were younger than patients in group 1 and 2 (44 years versus 51 and 48 years, respectively).

Thirty-nine patients presented with neurosarcoidosis manifestations from more than one category (Figure 3.1). There were seven patients with neurosarcoidosis manifestations from all three categories.

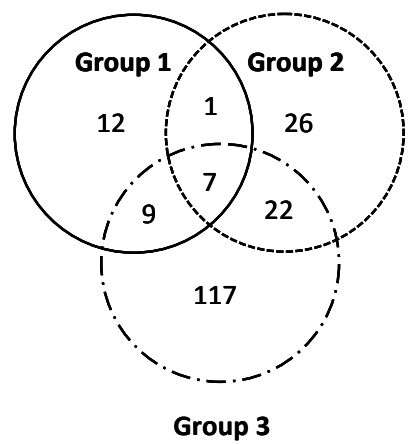

Figure 3.1 Venn diagram of manifestations from different neurosarcoidosis categories (Group 1: polyneuropathy, Group 2: spinal cord involvement and Group 3: cerebral involvement).

\section{Group 1: polyneuropathy}

Hypercalcaemia was seen more frequently in patients with group 1 manifestations than in the other two groups ( $14 \%$ vs. $2 \%, p=0.006)$. Patients with polyneuropathy had less cerebral involvement ( $7 \%$ vs. $29 \%, p=0.01$ ). Regarding treatment, patients with polyneuropathy were more often treated with methotrexate than patients in groups 2 and 3 (69 \% vs. $42 \%, p=0.007$; Table 3.3 ).

\section{Group 2: spinal cord disease}

Peripheral lymph node involvement was observed more frequently (93\% vs. $79 \%$, $p=0.02$ ) in this subgroup compared to group 1 and 3 . No neuro-endocrine or cerebrovascular involvement was observed in patients with spinal cord manifestations $(p=0.002$ and $p=0.015$, respectively). Cranial nerve dysfunction and chronic aseptic meningitis were also less common among patients with manifestations in this group ( $19.6 \%$ vs. $47.1 \%, p \leq 0.001$ and $27.7 \%$ vs. $47.8 \%, p=0.011$, respectively). By contrast, 
there was more brainstem involvement ( $11.1 \%$ vs. $3.6 \%, p=0.045)$. Infliximab had been started in a relatively high proportion of patients with manifestations in this group compared to the other categories, although this difference was not significant ( $29 \%$ vs. 17\%, $p=0.07$; Table 3.3).

Group 3: cerebral parenchymal lesions, neuro-endocrine, and brainstem involvement, cerebral infarction, chronic aseptic meningitis, hydrocephalus and cranial nerve manifestations

No differences in systemic manifestations were observed between group 3 patients and the rest (group 1 and 2).

Patients with manifestations in this group had less frequently received treatment with methotrexate ( $42 \%$ vs. $62 \%, p=0.03$ ) compared to the other groups (Table 3.3 ). Adalimumab was only given to patients in this group $(n=4)$.

Table 3.3 Treatment for different groups of neurosarcoidosis manifestations.

\begin{tabular}{lccc}
\hline & Group 1 $(\mathrm{n}=29)$ & Group 2 $(\mathrm{n}=56)$ & Group 3 $(\mathrm{n}=155)$ \\
\hline prednisone & $26(90)$ & $51(91)$ & $140(90)$ \\
methotrexate & $20(69)$ & $30(54)$ & $65(42)$ \\
azathioprine & $4(14)$ & $5(9)$ & $21(14)$ \\
mycophenolate mofetil & $0(0)$ & $3(5)$ & $4(3)$ \\
cyclosporine & $0(0)$ & $0(0)$ & $1(0.6)$ \\
cyclophosphamide & $0(0)$ & $1(2)$ & $1(0.6)$ \\
infliximab & $7(24)$ & $16(29)$ & $32(21)$ \\
adalimumab & $0(0)$ & $0(0)$ & $4(3)$ \\
\hline
\end{tabular}

\section{Discussion}

This study has been one of the few neurosarcoidosis cohort studies so far, and was performed in two Dutch national expertise centres and a national neurosarcoidosis database. Both expertise centres showed similar results in terms of prevalence of the manifestations. The most common neurosarcoidosis manifestations were chronic aseptic meningitis (42\%), cranial nerve dysfunction (39\%), spinal cord involvement (28\%) and cerebral parenchymal disease (26\%). Strikingly, much overlap was found in manifestations, with $56 \%$ of patients having two or more manifestations simultaneously. Over $90 \%$ of patients needed treatment.

The clinical presentation of neurosarcoidosis is known to be heterogeneous. ${ }^{18}$ The disorder is hard to recognize due to its heterogeneity and rarity. We also found this diversity of clinical presentations in our Dutch cohort of neurosarcoidosis patients. We 
report a higher prevalence of meningitis, viz. $41 \%$, versus $16 \%$ reported in a metaanalysis. ${ }^{9}$ This could probably be explained by the fact that both centres in our study are tertiary referral centres (inclusion bias). The Amsterdam UMC is also a referral centre for inflammatory and infectious meningitis. We also found more spinal cord involvement (28\% versus $18 \%){ }^{9}$ These manifestations are found in severely affected neurosarcoidosis patients, which is the main reason for referral. By contrast, we found less cranial nerve involvement (39\% versus $55 \%)$, ${ }^{9}$ with the facial, optic, trigeminal and vestibulocochlear nerves as the most commonly involved nerves. It is highly likely that these differences can be explained by the small sample sizes and mainly retrospective design of these previous studies, ${ }^{9}$ and by the tertiary setting of our neurosarcoidosis population. A comparison between our results and those reported from other referral centres showed that results were similar. ${ }^{16,24,33}$ The prevalence of peripheral polyneuropathy in our study was comparable to that reported in a large meta-analysis. ${ }^{9}$ Race and gender distributions in our total cohort of neurosarcoidosis patients were also similar to those found in previous studies. ${ }^{9}$

Manifestations of sarcoidosis are known to differ between races and genders. ${ }^{10,27-30,34-40}$ Men with sarcoidosis tend to have higher rates of cardiac and pulmonary involvement, whereas women have a higher prevalence of peripheral lymph node involvement, cutaneous, musculoskeletal, liver, spleen and ocular involvement. ${ }^{10,30,34-38}$ Furthermore, hypercalcaemia tends to be more prevalent in men. ${ }^{38}$ In the Dutch neurosarcoidosis sample studied, the prevalence of various neurosarcoidosis manifestations did not differ between genders, except for peripheral polyneuropathy which was more common in men than in women $(21.1 \%$ versus $9.6 \%)$. This finding is in line with other auto-immune polyneuropathies, ${ }^{41-45}$ in contrast to diabetic polyneuropathy, where no gender differences were found. ${ }^{46}$ The explanation for this finding remains unclear.

As regards race, the ACCESS study, a large case-control study of sarcoidosis, found that patients of African descent were more likely to have cutaneous involvement, as well as erythema nodosum, extrathoracic lymph node, ocular, liver and bone marrow involvement, while Caucasians were more likely to develop hypercalcemia and spleen involvement. ${ }^{10,39}$ In a self-reported organ involvement registry, Baughman et al. did not find differences in the prevalence of neurosarcoidosis in general between genders or races. ${ }^{11}$ In our neurosarcoidosis sample, patients of African descent appeared to be overrepresented $(21 \%)$ in comparison with the general Dutch population $(4 \%)$. This might be explained by the fact that the population in urban areas of the Netherlands (the west of the country [including Amsterdam]) and to a lesser extent the centre (e.g. Nieuwegein) includes more people of non-Dutch origin, causing bias in the racial distribution in our cohort. Moreover, patients of African descent have a higher risk of developing a more chronic severe course of sarcoidosis. However, no differences 
between races were found in our neurosarcoidosis sample regarding the prevalence of the various neurosarcoidosis manifestations.

Differences between races and genders regarding the various manifestations of sarcoidosis need to be considered in the management of this disorder. One of the main difficulties in accurately identifying patients at risk of progressive major organ damage and death is the heterogeneity of its presentation. Thus, the optimal risk stratification strategy in the general sarcoidosis population continues to be unclear. ${ }^{47}$ The identification of patients at higher risk of eventual major organ involvement, in order to initiate timely treatment to prevent progression, remains a clinical challenge. The integration of clinical symptoms, results of functional and imaging examinations, and observed disease behaviour in the definition of severe sarcoidosis seems to be the most comprehensive approach suggested to date. ${ }^{48}$ Studies in the United States found sarcoidosis mortality to be related to both race and gender, perhaps reflecting genetic factors. African-American individuals and women had a worse prognosis, with a higher prevalence of extra-pulmonary organ involvement and prolonged disease duration, and a higher rate of sarcoidosis-related hospitalizations. ${ }^{27-29,49}$ Sarcoidosis patients of African descent also had a higher mortality rate than Caucasians and were treated more often. ${ }^{10,29}$ In a large neurosarcoidosis study by Joubert et al. ( $\left.n=234\right)$, no differences in mortality rates were found between various ethnicities. ${ }^{16}$ The mortality difference between Caucasians and patients of African descent with sarcoidosis is thus explained by other sarcoidosis manifestations than neurosarcoidosis, for instance pulmonary hypertension and cardiac and more advanced pulmonary sarcoidosis. ${ }^{10,29,47}$ The majority of patients in the studied Dutch neurosarcoidosis sample needed treatment (93\%). This underlines that treatment is mandatory for neurosarcoidosis, and in many cases second- or third-line treatment is required (53\%). Considering this, plus the high relapse rate necessitating long-term treatment for neurosarcoidosis and the many side-effects of corticosteroids, ${ }^{16,33,50,51}$ second- or third-line agents (methotrexate, azathioprine, or TNF- $\alpha$ inhibitors) are preferable as the first-choice treatment in selected neurosarcoidosis cases, especially in patients with comorbidities such as obesity or diabetes. Based on clinical experience with other sarcoidosis manifestations and previous studies, second-line treatment with azathioprine and methotrexate can be regarded as safe and effective. ${ }^{52}$ TNF- $\alpha$ inhibitors have shown efficacy and safety for both sarcoidosis in general and neurosarcoidosis. ${ }^{23,53-60}$ Moreover, lower relapse rates of neurosarcoidosis have been described with infliximab and methotrexate, ${ }^{16,33}$ as well as high relapse rates of sarcoidosis and neurosarcoidosis after discontinuation of infliximab. Thus, the decision to discontinue should be made very carefully and longterm continuation should be considered. ${ }^{23,53,54,61,62}$ Previously, treatment with TNF- $\alpha$ inhibitors led to high health care costs. The expiring of the patent of Remicade ${ }^{\circledR}$ 
(Infliximab) and the availability of biosimilars (Inflectra ${ }^{\circledR}$ ) with similar efficacy have now reduced the costs. ${ }^{63}$

Our study had several limitations. Despite significant advances in diagnostics of neurosarcoidosis made in the last two decades, no consensus has been achieved about its classification. We used the most widely used modified Zajicek criteria. Follow-up of neurosarcoidosis is difficult, since no sensitive and specific biomarker is available so far. Disease assessment is often based on symptoms and neurologic examination, sometimes combined with follow-up cerebral or spinal MRI's (when applicable), making it hard to assess whether the disease is truly in remission. Another limitation could be a selection bias, since most of our patients were diagnosed and treated in tertiary expertise centres. However, patients with suspected neurosarcoidosis are usually referred to such expertise centres because of the complexity of diagnosing and treating this rare disease entity. Another limitation is the observational nature of our study, with some missing values and no structured monitoring and follow-up. Since neurosarcoidosis is a rare sarcoidosis manifestation, however, it is difficult to perform studies with another study design.

\section{Conclusion}

Chronic aseptic meningitis, cranial nerve dysfunction, spinal cord involvement and cerebral parenchymal disease appeared to be the most common neurosarcoidosis manifestations, without racial or gender differences. Treatment was mandatory in the majority of patients, $>50 \%$ of whom required second- or third-line treatment. The current risk stratification strategy to identify severe neurosarcoidosis patients involves a multidisciplinary approach integrating symptoms, functional tests and imaging findings. Future studies are warranted to enhance our understanding of the diversity of neurosarcoidosis, the determinants of morbidity, severity and mortality, as well as optimal treatment regimens, in order to determine the best treatment to improve the quality of life of patients suffering from this rare form of sarcoidosis. 


\section{References}

1. Arkema EV, Grunewald J, Kullberg S, et al. Sarcoidosis incidence and prevalence: a nationwide registerbased assessment in Sweden. Eur Respir J 2016;48(6):1690-9.

2. Thomas KW, Hunninghake GW. Sarcoidosis. JAMA 2003;289(24):3300-3.

3. Durel CA, Marignier R, Maucort-Boulch D, et al. Clinical features and prognostic factors of spinal cord sarcoidosis: a multicenter observational study of 20 biopsy-proven patients. J Neurol 2016;263(5): 981-90.

4. Gerke AK, Hunninghake G. The immunology of sarcoidosis. Clin Chest Med. 2008;29(3):379-90, vii.

5. Drent M, Lower EE, De Vries J. Sarcoidosis-associated fatigue. Eur Respir J 2012;40(1):255-63.

6. Statement on sarcoidosis. Joint Statement of the American Thoracic Society (ATS), the European Respiratory Society (ERS) and the World Association of Sarcoidosis and Other Granulomatous Disorders (WASOG) adopted by the ATS Board of Directors and by the ERS Executive Committee, February 1999. Am J Respir Crit Care Med 1999;160(2):736-55.

7. Drent M, Strookappe B, Hoitsma E, De Vries J. Consequences of sarcoidosis. Clin Chest Med 2015;36(4):727-37.

8. Valeyre D, Prasse A, Nunes H, et al. Sarcoidosis. Lancet 2014;383(9923):1155-67.

9. Fritz D, van de Beek D, Brouwer MC. Clinical features, treatment and outcome in neurosarcoidosis: systematic review and meta-analysis. BMC Neurol 2016;16(1):220.

10. Judson MA, Boan AD, Lackland DT. The clinical course of sarcoidosis: presentation, diagnosis, and treatment in a large white and black cohort in the United States. Sarcoidosis Vasc Diffuse Lung Dis 2012;29(2):119-27.

11. Baughman RP, Culver DA, Judson MA, et al. Self-reported organ involvement in sarcoidosis: results of a multinational registry. Am J Resp Crit Care Med 2018;197:A7482.

12. Ungprasert $P$, Crowson CS, Matteson EL. Characteristics and long-term outcome of neurosarcoidosis: a population-based study from 1976-2013. Neuroepidemiology 2017;48(3-4):87-94.

13. Iannuzzi MC, Rybicki BA, Teirstein AS. Sarcoidosis. N Engl J Med 2007;357(21):2153-65.

14. Joseph FG, Scolding NJ. Sarcoidosis of the nervous system. Pract Neurol 2007;7(4):234-44.

15. Marangoni S, Argentiero V, Tavolato B. Neurosarcoidosis. Clinical description of 7 cases with a proposal for a new diagnostic strategy. J Neurol 2006;253(4):488-95.

16. Joubert B, Chapelon-Abric C, Biard L, et al. Association of prognostic factors and immunosuppressive treatment with long-term outcomes in neurosarcoidosis. JAMA Neurol 2017;74(11):1336-44.

17. Pawate S, Moses H, Sriram S. Presentations and outcomes of neurosarcoidosis: a study of 54 cases. QJM 2009;102(7):449-60.

18. Fritz $D$, Voortman $M$, van de Beek $D$, et al. Many faces of neurosarcoidosis: from chronic meningitis to myelopathy. Curr Opin Pulm Med 2017;23(5):439-46.

19. Joseph FG, Scolding NJ. Neurosarcoidosis: a study of 30 new cases. J Neurol Neurosurg Psychiatry 2009;80(3):297-304.

20. Shah R, Roberson $\mathrm{GH}$, Cure JK. Correlation of $M R$ imaging findings and clinical manifestations in neurosarcoidosis. Am J Neuroradiol 2009;30(5):953-61.

21. Stern BJ, Krumholz A, Johns C, et al. Sarcoidosis and its neurological manifestations. Arch Neurol 1985;42(9):909-17.

22. Zajicek JP, Scolding NJ, Foster O, et al. Central nervous system sarcoidosis: diagnosis and management. QJM 1999;92(2):103-17.

23. Cohen Aubart F, Bouvry D, Galanaud D, et al. Long-term outcomes of refractory neurosarcoidosis treated with infliximab. J Neurol 2017;264(5):891-7.

24. Leonhard SE, Fritz D, Eftimov F, et al. Neurosarcoidosis in a tertiary referral center: a cross-sectional cohort study. Medicine (Baltimore) 2016;95(14):e3277.

25. Langrand C, Bihan H, Raverot G, et al. Hypothalamo-pituitary sarcoidosis: a multicenter study of 24 patients. QJM 2012;105(10):981-95.

26. Scott TF, Yandora K, Valeri A, et al. Aggressive therapy for neurosarcoidosis: long-term follow-up of 48 treated patients. Arch Neurol 2007;64(5):691-6. 
27. Gerke AK, Judson MA, Cozier YC, et al. Disease burden and variability in sarcoidosis. Ann Am Thorac Soc 2017;14(Supplement_6):S421-S8.

28. Kirkil G, Lower EE, Baughman RP. Predictors of mortality in pulmonary sarcoidosis. Chest 2018;153(1):105-13.

29. Mirsaeidi M, Machado RF, Schraufnagel D, et al. Racial difference in sarcoidosis mortality in the United States. Chest 2015;147(2):438-49.

30. Ungprasert $\mathrm{P}$, Crowson CS, Matteson EL. Influence of gender on epidemiology and clinical manifestations of sarcoidosis: a population-based retrospective cohort study 1976-2013. Lung 2017;195(1):87-91.

31. www.neurosarcoidose.nl.

32. Voortman M, Fritz D, Vogels OJM, et al. Small fiber neuropathy: a disabling and underrecognized syndrome. Curr Opin Pulm Med 2017;23(5):447-57.

33. Bitoun S, Bouvry D, Borie R, et al. Treatment of neurosarcoidosis: a comparative study of methotrexate and mycophenolate mofetil. Neurology 2016;87(24):2517-21.

34. Brito-Zeron P, Sellares J, Bosch X, et al. Epidemiologic patterns of disease expression in sarcoidosis: age, gender and ethnicity-related differences. Clin Exp Rheumatol 2016;34(3):380-8.

35. Yanardag H, Pamuk ON, Karayel T. Cutaneous involvement in sarcoidosis: analysis of the features in 170 patients. Respir Med 2003;97(8):978-82.

36. Lill H, Kliiman K, Altraja A. Factors signifying gender differences in clinical presentation of sarcoidosis among Estonian population. Clin Respir J 2016;10(3):282-90.

37. Ungprasert P, Wetter DA, Crowson CS, Matteson EL. Epidemiology of cutaneous sarcoidosis, 19762013: a population-based study from Olmsted County, Minnesota. J Eur Acad Dermatol Venereol 2016;30(10):1799-804.

38. Birnbaum AD, Rifkin LM. Sarcoidosis: sex-dependent variations in presentation and management. J Ophthalmol 2014;2014:236905.

39. Baughman RP, Teirstein AS, Judson MA, et al. Clinical characteristics of patients in a case control study of sarcoidosis. Am J Respir Crit Care Med 2001;164(10 Pt 1):1885-9.

40. Birnbaum AD, Oh FS, Chakrabarti A, et al. Clinical features and diagnostic evaluation of biopsy-proven ocular sarcoidosis. Arch Ophthalmol 2011;129(4):409-13.

41. Chio $A$, Cocito $D$, Bottacchi $E$, et al. Idiopathic chronic inflammatory demyelinating polyneuropathy: an epidemiological study in Italy. J Neurol Neurosurg Psychiatry 2007;78(12):1349-53.

42. Lunn MP, Manji H, Choudhary PP, et al. Chronic inflammatory demyelinating polyradiculoneuropathy: a prevalence study in south east England. J Neurol Neurosurg Psychiatry 1999;66(5):677-80.

43. Mygland A, Monstad P. Chronic polyneuropathies in Vest-Agder, Norway. Eur J Neurol 2001;8(2): 157-65.

44. Rotta FT, Sussman AT, Bradley WG, et al. The spectrum of chronic inflammatory demyelinating polyneuropathy. J Neurol Sci 2000;173(2):129-39.

45. Chandra SR, Karru VR, Mukheem Mudabbir MA, et al. Immune-mediated neuropathies our experience over 3 Years. J Neurosci Rural Pract 2018;9(1):30-5.

46. Aslam A, Singh J, Rajbhandari S. Prevalence of painful diabetic neuropathy using the self-completed leeds assessment of neuropathic symptoms and signs questionnaire in a population with diabetes. Can J Diabetes 2015;39(4):285-95.

47. Walsh S WA, Kouranos V. Why do people die from sarcoidosis? Curr Opin Pulm Med 2018;24(5):527535.

48. Kouranos V, Jacob J, Wells AU. Severe sarcoidosis. Clin Chest Med 2015;36(4):715-26.

49. Tukey MH, Berman JS, Boggs DA, et al. Mortality among African American women with sarcoidosis: data from the Black women's health study. Sarcoidosis Vasc Diffuse Lung Dis 2013;30(2):128-33.

50. Khan NA, Donatelli CV, Tonelli AR, et al. Toxicity risk from glucocorticoids in sarcoidosis patients. Respir Med 2017;132:9-14.

51. Broos CE, Poell LHC, Looman CWN, et al. No evidence found for an association between prednisone dose and FVC change in newly-treated pulmonary sarcoidosis. Respir Med 2018;138S:S31-S7.

52. Vorselaars ADM, Wuyts WA, Vorselaars VMM, et al. Methotrexate vs azathioprine in second-line therapy of sarcoidosis. Chest 2013;144(3):805-12. 
53. Chintamaneni S, Patel AM, Pegram SB, et al. Dramatic response to infliximab in refractory neurosarcoidosis. Ann Indian Acad Neurol 2010;13(3):207-10.

54. Gelfand JM, Bradshaw MJ, Stern BJ, et al. Infliximab for the treatment of CNS sarcoidosis: a multiinstitutional series. Neurology 2017;89(20):2092-100.

55. Moravan M, Segal BM. Treatment of CNS sarcoidosis with infliximab and mycophenolate mofetil. Neurology 2009;72(4):337-40.

56. Sodhi M, Pearson K, White ES, Culver DA. Infliximab therapy rescues cyclophosphamide failure in severe central nervous system sarcoidosis. Respir Med 2009;103(2):268-73.

57. Wijnen PA, Cremers JP, Nelemans PJ, et al. Association of the TNF-alpha G-308A polymorphism with TNF-inhibitor response in sarcoidosis. Eur Respir J 2014;43(6):1730-9.

58. Vorselaars AD, Crommelin HA, Deneer VH, et al. Effectiveness of infliximab in refractory FDG PETpositive sarcoidosis. Eur Respir J 2015;46(1):175-85.

59. Judson MA, Baughman RP, Costabel $U$, et al. Efficacy of infliximab in extrapulmonary sarcoidosis: results from a randomised trial. Eur Respir J 2008;31(6):1189-96.

60. Hostettler KE, Studler U, Tamm M, Brutsche MH. Long-term treatment with infliximab in patients with sarcoidosis. Respiration 2012;83(3):218-24.

61. Vorselaars AD, Verwoerd A, van Moorsel $\mathrm{CH}$, et al. Prediction of relapse after discontinuation of infliximab therapy in severe sarcoidosis. Eur Respir J 2014;43(2):602-9.

62. Panselinas E, Rodgers JK, Judson MA. Clinical outcomes in sarcoidosis after cessation of infliximab treatment. Respirology 2009;14(4):522-8.

63. Schimmelpennink MC, Vorselaars ADM, van Beek FT, et al. Efficacy and safety of infliximab biosimilar Inflectra((R)) in severe sarcoidosis. Respir Med 2018;138S:S7-S13. 



\section{Chapter 4}

\section{Everyday cognitive failure in patients suffering}

from neurosarcoidosis

M. Voortman, J. De Vries, C.M.R. Hendriks, M.D.P. Elfferich, P.A.H.M. Wijnen, M. Drent Sarcoidosis Vasculitis and Diffuse Lung Diseases 2019; 36 (1); 2-10. 


\section{Abstract}

\section{Background}

Cognitive failure is associated with memory and concentration problems. Previously, a prevalence of one third was found in a general sarcoidosis population. The aim of this study was to assess if neurosarcoidosis patients are at higher risk for developing everyday cognitive failure using the Cognitive Failure Questionnaire (CFQ) and to determine what factors were associated with cognitive failure.

\section{Methods}

A cross-sectional web-based survey was conducted from April to May 2017 in a national sample of neurosarcoidosis patients. The survey asked about complaints and included 3 questionnaires (Fatigue Assessment Scale [FAS], Small Fiber Neuropathy Screening List [SFNSL] and CFQ). Data were compared to a general sarcoidosis population.

\section{Results}

Of the 152 patients who completed the survey, 131 had neurosarcoidosis. The mean CFQ score was significantly higher in the neurosarcoidosis $(45.6 \pm 20.7)$ compared to the general sarcoidosis population $(36.2 \pm 15.9 ; p<0.0001)$. High CFQ scores $(\geq 43)$ were found in $55.7 \%$ and $33.9 \%$, respectively $(p<0.0001)$. The FAS score (OR 21.4 ) and SFNSL score (OR 4.3) were the strongest positive predictors of a high CFQ score.

\section{Conclusion}

Cognitive failure is a significant problem in neurosarcoidosis. More than half of the patients reported cognitive deficits, compared to one third of a general sarcoidosis population. Fatigue and small fiber neuropathy play a role in cognitive failure. 


\section{Introduction}

Cognitive failure is a cognitive error occurring during the performance of a task that a person would normally execute successfully in everyday life. ${ }^{1,2}$ Cognitive failure is characterized by concentration problems, memory loss and decreased perception. It is associated with various disease entities, such as chronic obstructive pulmonary disease, obstructive sleep apnoea, chronic heart failure, rheumatoid arthritis, primary Sjögren syndrome, multiple sclerosis and sarcoidosis. Factors associated with a higher prevalence of cognitive failure in these different disease entities include fatigue, depression, pain due to small fiber neuropathy (SFN), microangiopathy, inflammatory molecules such as tumour necrosis factor-alpha (TNF- $\alpha$ ), cerebrovascular disease and chronic hypoxia. ${ }^{1,3-7}$

Sarcoidosis is a multisystem inflammatory disorder of unknown aetiology in genetically predisposed individuals, affecting 1-40 per 100000 persons. ${ }^{8,9}$ The natural history and prognosis of sarcoidosis are highly variable and its course is often unpredictable. Clinical manifestations vary, depending on the organs involved. ${ }^{10,11}$ Involvement of the nervous system, neurosarcoidosis, is present in approximately $5 \%$ of the cases and has a heterogeneous clinical presentation, from chronic meningitis to myelopathy. ${ }^{12,13}$ Apart from organ-related symptoms, patients may suffer from a variety of nonspecific disabling symptoms that cannot be explained by granulomatous inflammation of an organ, such as fatigue and small fiber neuropathy (SFN). ${ }^{14}$ Both have a high prevalence. Fatigue occurs in $50-85 \%$ of sarcoidosis patients ${ }^{11,15}$ and SFN in $40-60 \% .{ }^{16,17}$ In addition, Elfferich et al. found everyday cognitive failure in $35 \%$ of the cases they studied in a general sarcoidosis population. ${ }^{1}$ Cognitive failure has a great impact on the lives of sarcoidosis patients, since they are mostly young.

Broadbent et al. developed the Cognitive Failure Questionnaire (CFQ), a self-report questionnaire assessing failures in everyday errors of attention, perception, memory and motor function. ${ }^{2}$ In general, the CFQ appears to be a reliable and brief measure useful in clinical practice.

We hypothesized that everyday cognitive functioning may be even more impaired in patients with neurosarcoidosis. The aim of this study was therefore to examine the prevalence of everyday cognitive failure using the CFQ in patients suffering from neurosarcoidosis compared to a general sarcoidosis population. We also studied what factors were associated with cognitive failure. 


\section{Methods}

\section{Study design}

A cross-sectional web-based anonymous survey was conducted from April to May 2017 among a national sample of neurosarcoidosis patients (Group I). The recruitment procedure aimed to compose a representative sample of neurosarcoidosis patients in the Netherlands. Data from the study by Elfferich et al., performed in a general sarcoidosis population $(n=343$ ) served as a control group for comparisons of the scores on the cognitive failure questionnaire [CFQ], the small fiber neuropathy screening list [SFNSL], and the fatigue assessment scale [FAS]. ${ }^{1}$ This latter population was subdivided in those without neurosarcoidosis (Group II, $\mathrm{n}=330$ ) and those with neurosarcoidosis (Group III, $\mathrm{n}=13$ ).

This study was performed in accordance with the Declaration of Helsinki and its amendments. The Medical Ethics Committee of the St. Antonius Hospital Nieuwegein, The Netherlands, decided that, under the Dutch act on medical research involving human subjects, approval of this study by a Medical Ethics Committee was not necessary.

\section{Study sample and procedure}

The overall study sample (group I) comprised patients from our online Dutch Neurosarcoidosis Registry and neurosarcoidosis patients from the Dutch Sarcoidosis Society. The diagnosis of neurosarcoidosis was confirmed for each of the patients by a neurologist, using the Zajicek or Marangoni (modified Zajicek) criteria, labelling patients as possible, probable or definite neurosarcoidosis. ${ }^{18,19}$ SFN was classified as 'paraneurosarcoidosis', since the hallmark of sarcoidosis (granuloma formation) is not found in the small fibers. ${ }^{20-23}$ Patients suffering solely from SFN were therefore excluded from this study.

All patients included in the Dutch Neurosarcoidosis Registry have agreed to participate in online research studies. Patients from the Dutch Sarcoidosis Society (Sarcoidose.nl) were recruited without incentives, since the survey was anonymous. There was no overlap in registrations between the two sources. All patients had sufficient command of Dutch and had access to the internet.

A survey was developed using the online questionnaire tool Surveymonkey (www.surveymonkey.com). The survey included self-reported complaints, sarcoidosis manifestations, demographics (gender, age, duration of sarcoidosis), use of medication, daily impairments and a set of questionnaires validated for sarcoidosis, the CFQ, ${ }^{2}$ the 
SFNSL $^{24}$ and the FAS. ${ }^{25}$ An invitation to complete the electronic survey was sent by email to all the patients in our online Dutch Neurosarcoidosis Registry. Additional patients were recruited by means of an advertisement on the website of the Dutch Sarcoidosis Society (sarcoidose.nl), providing a link to the electronic survey. Of the 153 patients who started the survey, 152 (99\%) completed it.

\section{Questionnaires}

In the sarcoidosis population, so far no questionnaires regarding evaluation of cognitive failure are validated. Broadbent et al. developed the CFQ to evaluate cognitive failure, which appears to be a brief and reliable questionnaire that is useful in clinical practice (see Appendix). Since this questionnaire was previously used to evaluate cognitive failure in the general sarcoidosis population, we decided to also use the CFQ in the neurosarcoidosis population. ${ }^{1}$ The CFQ is a self-report questionnaire consisting of 25 items assessing deficits regarding attention, perception, memory and motor functioning in everyday life. The total CFQ score is calculated by summation of all answers and scores range from 0-100. A higher total score indicates more subjective cognitive failure. A high CFQ score is defined as a score $\geq 43$ (mean of the controls plus one standard deviation). ${ }^{2}$

The FAS is a 10-item self-report fatigue questionnaire. In addition to the total fatigue score, the FAS yields a mental fatigue score and a physical fatigue score (see Appendix). The response scale is a five-point scale ( 1 never to 5 always); scores on the FAS can range from 10 to 50 . A score $>22$ indicates fatigue and a score $>34$ indicates extreme fatigue. The reliability and validity of the FAS have been shown to be good in sarcoidosis patients. ${ }^{25}$

The SFNSL is a 21-item self-administered questionnaire to screen for symptoms related to small fiber neuropathy (SFN; see Appendix). The response scale is a five-point scale ( 0 never to 4 always); scores on the SFNSL can range from 0 to 84 . The cut-off score of the SFNSL is 11: a score below 11 indicates no or few symptoms related to SFN, while a score of 11-48 indicates probable or highly probable SFN and a score above 48 is indicative of SFN. ${ }^{24}$

\section{Statistical analysis}

All statistical analyses were performed using SPSS version 24 for Mac. Standard descriptive statistics were computed. The chi-square test and Student t-test were used to test for statistically significant differences between two groups of neurosarcoidosis 
patients (i.e. those with $\mathrm{CFQ}<43$ and those with $\mathrm{CFQ} \geq 43$ ). Chi-square tests were also performed to assess the prevalence (\%) of cognitive failure (CFQ) in patients with neurosarcoidosis compared to a general sarcoidosis population. ANOVA was used for comparison between the general sarcoidosis population and the neurosarcoidosis population. Pearson correlations were calculated to evaluate the association between the CFQ scores and fatigue (FAS scores) as well as symptoms suggestive of SFN (SFNSL scores). Multivariate logistic regression analyses were used to assess the influence of the FAS scores and SFNSL scores on the CFQ scores, after adjustment for age, sex, treatment, depressive symptoms and sleeping disturbances. Because of the large number of correlations examined, a probability value of less than 0.01 was considered to be statistically significant.

\section{Results}

A total of 152 patients (99\%) completed the survey. Neurosarcoidosis had been established in 131 patients (86.2\%), 12 patients did not have neurosarcoidosis, and in 9 cases the diagnosis remained unclear. Finally, therefore, 131 patients suffering from neurosarcoidosis were included in this study. The studied population is approximately $25 \%$ of the estimated total neurosarcoidosis population in the Netherlands. The various neurosarcoidosis manifestations in the studied population were cranial nerve palsy (45.1\%), spinal cord involvement (29.4\%), chronic meningitis (26.5\%), cerebral involvement (20.6\%), peripheral neuropathy (15.7\%), myelitis (9.8\%), neuro-endocrine involvement (9.8\%), hydrocephalus (6.9\%), brainstem involvement (4.9\%), and cerebral vascular involvement (3.9\%). Almost half of the population had multiple manifestations (45\%). Demographic and clinical data are summarized in Table 4.1.

The mean CFQ score in the neurosarcoidosis sample we studied was $45.6 \pm 20.7$. There were no significant differences between the subgroup with a high CFQ score and the subgroup with a normal CFQ score as regards sex, age, time since diagnosis, or medication use. Pain, pulmonary symptoms (cough and dyspnea), self-reported depressive symptoms and self-reported sleeping disturbances were more prevalent in the group with a high CFQ score (see Table 4.1). 
Table 4.1 Summary of the characteristics of the studied neurosarcoidosis population subdivided into those with normal Cognitive Failure Questionnaire (CFQ) scores and those with high CFQ scores.

\begin{tabular}{|c|c|c|c|c|}
\hline & Total & CFQ $<43$ & $\mathrm{CFQ} \geq 43$ & p-values\# \\
\hline patients n (\%) & 131 & $58(44.3)$ & $73(55.7)$ & - \\
\hline sex (male:female), n (\%) & $67(51.1): 64(48.9)$ & $27(46.6): 31(53.4)$ & $40(54.8): 33(45.2)$ & 0.349 \\
\hline age, years (range) & $51.9 \pm 10.4(21-75)$ & $52.8 \pm 11(21-75)$ & $51.4 \pm 10.0(28-74)$ & 0.444 \\
\hline time since diagnosis, years (range) & $8.7 \pm 7.2(0-35)$ & $8.0 \pm 6.7(0-35)$ & $9.3 \pm 7.6(1-35)$ & 0.292 \\
\hline \multicolumn{5}{|l|}{ symptoms } \\
\hline pain, $\mathrm{n}(\%)$ & $92(70.2)$ & $32(55.2)$ & $60(82.2)$ & 0.001 \\
\hline cough and dyspnea, n (\%) & 30 (22.9) & $5(8.6)$ & $25(34.2)$ & 0.001 \\
\hline ocular, n (\%) & $57(43.5)$ & $21(36.2)$ & $36(49.3)$ & 0.133 \\
\hline cardiac, n (\%) & $9(6.9)$ & $4(6.9)$ & $5(6.8)$ & 0.992 \\
\hline skin, n (\%) & $48(33.6)$ & $15(25.9)$ & $31(42.5)$ & 0.048 \\
\hline joint, n (\%) & 89 (67.9) & $33(56.9)$ & $56(76.7)$ & 0.016 \\
\hline hypercalcemia, n (\%) & $9(6.9)$ & $2(3.4)$ & $7(9.6)$ & 0.168 \\
\hline kidney stones, n (\%) & $3(2.3)$ & $0(0)$ & $3(4.1)$ & 0.118 \\
\hline \multicolumn{5}{|l|}{ medication use } \\
\hline never, n (\%) & $26(24.4)$ & $12(20.7)$ & $14(19.2)$ & 0.829 \\
\hline first line treatment, n (\%) & 99 (75.6) & $43(74.1)$ & $56(76.7)$ & 0.733 \\
\hline \multicolumn{5}{|l|}{ (prednisone or dexamethasone) } \\
\hline $\begin{array}{l}\text { second line treatment, } \mathrm{n}(\%) \text {, } \\
\text { (methotrexate, azathioprine, } \\
\text { mycofenolate mofetyl) }\end{array}$ & $50(38.2)$ & $20(34.5)$ & $30(41.1)$ & 0.439 \\
\hline $\begin{array}{l}\text { third line treatment, } \mathrm{n}(\%) \text { (anti- } \\
\text { TNF- } \alpha \text { : adalimumab, infliximab) }\end{array}$ & $22(16.8)$ & $10(17.2)$ & $12(16.4)$ & 0.903 \\
\hline \multicolumn{5}{|l|}{ psychological variables } \\
\hline \multicolumn{5}{|l|}{ self-reported } \\
\hline depressive symptoms & $33(25.2)$ & $8(13.8)$ & $25(34.2)$ & 0.007 \\
\hline sleeping disturbances & $70(53.4)$ & $18(31)$ & $52(71.2)$ & $<0.0001$ \\
\hline \multicolumn{5}{|l|}{ questionnaires } \\
\hline CFQ score & $45.6 \pm 20.7$ & $27.0 \pm 10.9$ & $60.3 \pm 13.4$ & $<0.0001$ \\
\hline FAS total score & $34.8 \pm 8.0$ & $30.2 \pm 7.8$ & $38.5 \pm 6.3$ & $<0.0001^{*}$ \\
\hline FAS-score <22, n (\%) & $10(7.6)$ & $9(15.5)$ & $1(1.4)$ & \\
\hline FAS-score 22-34, n (\%) & $51(38.9)$ & $33(56.9)$ & $18(24.7)$ & \\
\hline FAS-score >34, n (\%) & $70(53.4)$ & $16(27.6)$ & $54(74)$ & \\
\hline FAS mental score & $15.8 \pm 4.4$ & $13.5 \pm 4.0$ & $17.6 \pm 3.9$ & $<0.0001$ \\
\hline FAS physical score & $19.0 \pm 4.1$ & $16.7 \pm 4.1$ & $20.8 \pm 2.9$ & $<0.0001$ \\
\hline SFNSL score & $39.7 \pm 21.3$ & $29.0 \pm 18.9$ & $48.3 \pm 19.3$ & $<0.0001^{*}$ \\
\hline SFNSL score $<11, \mathrm{n}(\%)$ & $11(8.4)$ & $10(17.2)$ & $1(1.4)$ & \\
\hline SFNSL score $11-48, \mathrm{n}(\%)$ & $69(52.7)$ & $38(65.5)$ & $31(42.5)$ & \\
\hline SFNSL score >48, n (\%) & $51(38.9)$ & $10(17.2)$ & $41(56.2)$ & \\
\hline
\end{tabular}

Data are expressed as means $\pm \mathrm{SD}, \mathrm{n}=$ absolute number or percentages. \#p-value CFQ $<43$ vs CFQ $\geq 43$, *p-value for total and subscores. TNF- $\alpha=$ tumour necrosis factor-alpha, CFQ=Cognitive Failure Questionnaire, FAS=Fatigue Assessment Scale, SFNSL=Small Fiber Neuropathy Screening List. 
The prevalence of a high CFQ score in the neurosarcoidosis sample (55.7\%) was significantly higher than in the general sarcoidosis population studied by Elfferich ( $n=330,33.9 \%, p<0.0001$; see Table 4.2) and comparable with the results in a subgroup of neurosarcoidosis patients $(n=13)$ in the study by Elfferich et al. (55.7\% versus $53.8 \%)^{1}$

Almost all neurosarcoidosis patients in our study reported fatigue $(n=121 ; 92.4 \%)$, $53.4 \%$ of whom experienced extreme fatigue (see Table 4.1). Fatigue was more prevalent in neurosarcoidosis patients with a high CFQ score $(98.6 \%$ vs. $84.5 \%$, respectively; $p \leq 0.0001)$. The average FAS scores were also higher in neurosarcoidosis with a high CFQ score (38.5 vs. 30.2 , respectively; $p \leq 0.0001$ ) The overall FAS scores, as well as the FAS scores in the high CFQ score subgroup, were significantly higher in the neurosarcoidosis population compared to the general sarcoidosis population (see Table 4.2). Symptoms suggestive of SFN (SFNSL score $>11$ ) were significantly more prevalent in the neurosarcoidosis patients with a high CFQ score $(98.6 \%$ vs $82.8 \%$, respectively; $\mathrm{p} \leq 0.0001)$. Comparison with the data of the general sarcoidosis population showed that the SFNSL scores in our study sample were comparable to those in the general sarcoidosis population and the high CFQ score subgroup (see Table 4.2).

Table 4.2 Scores on the Cognitive Failure Questionnaire (CFQ), Fatigue Assessment Scale (FAS), Small Fiber Neuropathy Screening List (SFNSL), gender and age of the neurosarcoidosis sample studied (Group I) and a general sarcoidosis patient population without neurosarcoidosis (Group II). ${ }^{1}$

\begin{tabular}{lccc}
\hline & Group I & Group II & p value \\
\hline patients $n$ & 131 & 330 & \\
gender: female $\%$ & 48.9 & 44.8 & NS \\
age, years (range) & $52.0 \pm 10.4(21-75)$ & $48.6 \pm 0.9(25-79)$ & NS \\
FAS score total group & $34.8 \pm 8.0$ & $29.2 \pm 8.4$ & 0.01 \\
FAS total score in CFQ $\geq 43$ & $38.5 \pm 6.3$ & $30.4 \pm 12.1$ & 0.001 \\
SFNSL score total group & $39.7 \pm 21.3$ & $42.4 \pm 16.8$ & $\mathrm{NS}$ \\
SFNSL score in CFQ $\geq 43$ & $48.3 \pm 19.3$ & $48.2 \pm 16.8$ & $\mathrm{NS}$ \\
CFQ score & $45.6 \pm 20.7$ & $36.2 \pm 15.9$ & $<0.0001$ \\
CFQ score $\geq 43, \mathrm{n}(\%)$ & $73(55.7)$ & $112(33.9)$ & $<0.0001$ \\
\hline
\end{tabular}

Data are expressed as means \pm SD, absolute number $(n)$ or percentages (\%). NS=not significant.

No statistically significant differences were found between the neurosarcoidosis sample we studied (Group I) and the subgroup of neurosarcoidosis patients included in the study by Elfferich et al. (Group III) ${ }^{1}$ as regards overall FAS, SFNSL and CFQ scores (data not shown). The average disease duration in our sample showed no statistically significant difference compared to the population of Elfferich. Disease duration did not 
influence everyday cognitive failure in sarcoidosis in this latter population and the population of the present study.

\section{Correlation of FAS, SFNSL and CFQ}

In our neurosarcoidosis population, both FAS and SFNSL scores correlated significantly with the CFQ. The FAS scores showed the strongest correlation with the CFQ $(R=0.65$; see Table 4.3). Patients with extreme fatigue (>34) appeared to be more at risk of developing cognitive failure than patients with a fatigue score $>22(R=0.46$ vs. $R=0.27$ respectively; $\mathrm{p} \leq 0.0001)$. Patients suffering from $\mathrm{SFN}$ related symptoms reported high CFQ scores ( $R=0.40, p \leq 0.0001)$.

Fatigue (OR 13.2) and symptoms suggestive of SFN (OR 5.5) were the strongest predictors of a high CFQ score. The OR of the SFNSL decreased after correction for sex, age, treatment, depressive symptoms and sleeping disturbances (OR 4.3, $p<0.0001$, $\mathrm{Cl}$ 2.7-11.3), while the OR of the FAS increased (OR 21.4, $\mathrm{p}=0.011, \mathrm{Cl} 2.0-225.2$ ) (see Table 4.4).

Table 4.3 Correlation of the scores on the Fatigue Assessment Scale (FAS) and Small Fiber Neuropathy (SFN) Screening List (SFNSL) with the Cognitive Failure Questionnaire (CFQ).

\begin{tabular}{lcccc}
\hline CFQ scores & FAS scores: total & FAS scores: mental & FAS scores: physical & SFNSL scores \\
\hline correlation ( $\mathrm{R}=$ ) & 0.65 & 0.60 & 0.64 & 0.53 \\
significance ( $p$-value) & $<0.0001$ & $<0.0001$ & $<0.0001$ & $<0.0001$ \\
CFQ scores & & FAS scores $>$ 22: & FAS scores >34: & SFNSL scores >48: \\
& & fatigue & extreme fatigue & highly likely SFN \\
correlation ( $\mathrm{R}=$ ) & & 0.27 & 0.46 & 0.40 \\
significance ( $p$-value) & & 0.002 & $<0.0001$ & $<0.0001$ \\
\hline
\end{tabular}

Table 4.4 Multivariate logistic regression Fatigue Assessment Scale (FAS) and Small Fiber Neuropathy Screening List (SFNSL) scores on the Cognitive Failure Questionnaire (CFQ) scores.

\begin{tabular}{llcc}
\hline & OR & $\mathbf{9 5 \% ~ C l}$ & p-value \\
\hline SFNSL & 4.3 & $2-11.3$ & $<0.0001$ \\
sleeping disturbances & 3.9 & $1.7-8.6$ & 0.001 \\
FAS & 21.4 & $2-225.2$ & 0.011 \\
age & 0.34 & $0.1-0.8$ & 0.016 \\
gender & 0.4 & $0.2-1$ & 0.048 \\
sleeping disturbances & 5.3 & $2.4-12$ & $<0.0001$ \\
\hline
\end{tabular}

OR=odds ratio, $95 \% \mathrm{Cl}=95 \%$ confidence interval. 


\section{Discussion}

To the best of our knowledge, this was the first study to examine everyday cognitive functioning in patients suffering from neurosarcoidosis and to compare this with a general sarcoidosis population using the cognitive failure questionnaire (CFQ). We found that everyday cognitive failure was a significant problem in the neurosarcoidosis population. More than half of the patients in our sample reported cognitive deficits, compared to one-third of a general sarcoidosis population studied by Elfferich and coworkers. ${ }^{1}$ Fatigue and symptoms of SFN were the most important predictors of cognitive failure.

Previously, it was demonstrated by Elfferich et al. that everyday cognitive failure is a substantial problem in sarcoidosis patients. ${ }^{1}$ In this latter study, no relation was found between the CFQ scores and inflammatory parameters, lung function test results, chest $\mathrm{X}$-ray stages, sleeping problems nor dyspnea. Moreover, no differences regarding these mentioned clinical data were shown between those patients with a high or normal CFQ score. In line with the latter study, the strongest predictor of cognitive failure in neurosarcoidosis was fatigue. Fatigue has been reported in $50-85 \%$ of chronic sarcoidosis patients ${ }^{11,15}$ and is also associated with poorer cognitive performance. ${ }^{1}$ Almost all our neurosarcoidosis patients experienced fatigue (FAS $\geq 22$ ), a higher proportion than in the general sarcoidosis population $(80.6 \%)$ studied by Elfferich et al.. ${ }^{1}$ Among the patients with cognitive failure, the neurosarcoidosis patients experienced more fatigue than sarcoidosis patients presenting with other manifestations. Fatigue in neurosarcoidosis patients has previously been described to affect cognitive control. ${ }^{26}$ In a study comparing non-fatigued and fatigued participants, the fatigued participants had compromised executive control. ${ }^{27}$ Studies in colorectal and breast cancer also found fatigue to be associated with perceived cognitive failure. $^{28,29}$ Moreover, fatigue negatively affects cognitive performance, in particular response inhibition. It may induce over-activation of the visual cortex, which is related to impaired cognitive performance. ${ }^{30}$ We did not find a difference between mental or physical fatigue: both were equally associated with cognitive failure in neurosarcoidosis patients.

SFN was also a predictor of cognitive failure, although to a lesser extent. The reported prevalence of SFN varies from 40 to $60 \%,{ }^{16,17}$ and has been associated with poorer cognitive performance in a general sarcoidosis population. ${ }^{1}$ SFN was even more prevalent in the neurosarcoidosis patient population we studied (91.6\%). One of the main symptoms of SFN is neuropathic pain. Previous studies have reported an 
association between chronic pain and cognitive deficits, including attention, working memory and executive function. ${ }^{31,32}$ A study of primary Sjögren's patients with SFN found a correlation between the intensity of pain and the performance of executive functions. ${ }^{6}$ Hendriks et al. found that everyday cognitive failure, and symptoms suggestive of SFN, appeared to be significant predictors of fatigue. ${ }^{33}$ Moreover, they also found that cognitive failure and depression are the most important predictors of high levels of fatigue. ${ }^{33}$ The study of Bosse-Henck et al. also determined depression, anxiety, muscle pain and severity of dyspnea were predictors of the development of severe fatigue. ${ }^{34}$ In line with this, we found a higher prevalence of depressive symptoms but also sleeping disturbances in neurosarcoidosis patients with cognitive failure correlating with the higher prevalence of fatigue. Moreover, sleeping disturbances are also associated with fatigue, depressive symptoms, anxiety, and dyspnea. $^{35,36}$

Cognition seems to be - at least partly - a consequence of fatigue and SFN in (neuro)sarcoidosis or due to a common underlying mechanism explaining the strong correlation between cognition, fatigue and SFN. In a pilot study by our group, standard neuropsychological tests were used to assess the cognitive domains of memory sensorimotor speed, information processing speed and cognitive flexibility. Only a small number of sarcoidosis patients ( $n=27 ; 63 \%$ female; age $47.2 \pm 10.8$ years) were tested and compared with healthy controls. They found that cognitive failure did not imply cognitive impairment. ${ }^{33}$ Thus, subjective cognitive failure was not associated with cognitive impairment. The latter study exemplifies the difficulties of the diagnostic classification of cognitive deficits in patients suffering from sarcoidosis without major morphological lesions, and emphasizes the necessity for further research in this field. Insight into cognitive functioning is of great importance to optimise the selfmanagement skills of patients with sarcoidosis. Indeed, cognitive deficits may lead to difficulties in managing their disease and negatively affect their treatment. Although cognitive dysfunction is a core feature of (neuro)sarcoidosis, most currently available treatments do not address cognition. ${ }^{1}$ Although treatment should first focus on treating sarcoidosis and its activity, ${ }^{1}$ alternatives could be considered if this is not effective.

The hallmark of sarcoidosis is granulomatous inflammation, which is predominantly a Thelper 1 immune response mediated by lymphocytes, macrophages and cytokines such as tumour necrosis factor alpha (TNF- $\alpha$ ) and interleukins (IL). ${ }^{37}$ It has been suggested that alterations in these immunological parameters can affect psychomotor functions. Research in mice has shown that TNF- $\alpha$ is essential for the normal functioning of memory and learning and that overexpression of TNF- $\alpha$ leads to cognitive failure, so 
TNF- $\alpha$ seems to have both neuroprotective and neurodegenerative effects. ${ }^{38,39}$ Previous research has demonstrated that TNF- $\alpha$ inhibition in a sarcoidosis population was the only treatment achieving improvement of CFQ score. ${ }^{1}$ Thus, overexpression of TNF- $\alpha$ in the pathogenesis of fatigue and cognitive failure could explain the favourable effect of TNF- $\alpha$ inhibitors in sarcoidosis patients on cognition and fatigue. ${ }^{1}$

The management of sarcoidosis patients with fatigue and low energy levels should focus on the increased burden of concomitant symptoms. Since fatigue usually has a multifactorial cause, risk factors should also be examined and treated in combination. ${ }^{14,33,34}$ Future research involving more comprehensive neuropsychological batteries is warranted to investigate psychological functioning and fatigue in sarcoidosis. In addition, further research should give more attention to possible mediating or confounding pathways and associations between everyday cognitive functioning, fatigue and SFN-related symptoms.

\section{Limitations of this study}

Our study was a cross-sectional study, in which the neuropsychological assessment of cognitive dysfunctions was restricted to a rather general and subjective screening instrument, the CFQ. It provides an impression of the prevalence and related factors. Labelling oneself as absent-minded or forgetful depends upon the perceived discrepancy between the subject's everyday memory functioning and her or his everyday memory demands. Self-reported cognitive changes or decline do not necessarily reflect actual cognitive decline, since subjective failure was not associated with cognitive impairment. ${ }^{33}$ Beliefs about cognitive changes are strongly influenced by self-efficacy beliefs, personality, vitality, the experience of daily functioning and coping styles. Nevertheless, it seems unlikely that the favourable effect on cognitive functioning reported by patients treated with TNF- $\alpha$-inhibitors is attributable to the use of a subjective screening instrument. ${ }^{1}$ Despite being subjective, therefore, this is the first sign that everyday cognitive failure is a major problem in neurosarcoidosis patients.

Response bias cannot be excluded due to the recruitment procedure, e.g. voluntary participation. Nevertheless, the response rate was high (99\%). The sample we studied is approximately $25 \%$ of the estimated total neurosarcoidosis population in the Netherlands, and therefore a rather representative reflection of this population.

Since neurosarcoidosis is a rare disorder with a great diversity of manifestations (with overlapping manifestations in $45 \%$ of cases), phenotyping aiming to perform statistical analysis would not have been valid or feasible due to the rather small sample sizes for 
each of the manifestations, unfortunately. However, it might be interesting to consider whether central neurological manifestations for instance expose more cognitive failure than peripheral ones.

\section{Conclusion}

Sarcoidosis patients, especially neurosarcoidosis patients, are at increased risk for developing everyday cognitive failure. More than half of the patients reported cognitive deficits, compared to one-third of a general sarcoidosis population. Cognitive problems in a relatively young patient population have a great impact on daily functioning and can lead to problems at work and in social life, leading to decreased quality of life. Fatigue and symptoms suggestive of small fiber neuropathy were the most important predictors of cognitive failure. Our study points towards the necessity to integrate the growing body of knowledge about neuropsychological deficits in sarcoidosis in the management of this disease, especially for those patients suffering from neurosarcoidosis. Further studies, including imaging and biomarkers, in furthering our understanding of cognitive failure in sarcoidosis are needed to determine the relationship between cognition, fatigue, sleepiness and small fiber neuropathy in sarcoidosis, and whether they might have a common pathogenesis. 


\section{References}

1. Elfferich MD, Nelemans PJ, Ponds RW, et al. Everyday cognitive failure in sarcoidosis: the prevalence and the effect of anti-TNF-alpha treatment. Respiration 2010;80:212-219.

2. Broadbent DE, Cooper PF, FitzGerald P, Parkes KR. The Cognitive Failures Questionnaire (CFQ) and its correlates. Br J Clin Psychol 1982;21(Pt 1):1-16.

3. Yohannes AM, Chen W, Moga AM. et al. Cognitive impairment in chronic obstructive pulmonary disease and chronic heart failure: a systematic review and meta-analysis of observational studies. J Am Med Dir Assoc 2017;18:451 e451-451 e411.

4. Montgomery-Downs HE, Crabtree VM, Gozal D. Cognition, sleep and respiration in at-risk children treated for obstructive sleep apnoea. Eur Respir J 2005;25:336-342.

5. Langdon DW. Cognition in multiple sclerosis. Curr Opin Neurol 2011;24:244-249.

6. Indart S, Hugon J, Guillausseau PJ, et al. Impact of pain on cognitive functions in primary Sjogren syndrome with small fiber neuropathy: 10 cases and a literature review. Medicine (Baltimore) 2017; 96:e6384.

7. Bartolini $\mathrm{M}$, Candela M, Brugni $\mathrm{M}$, et al. Are behaviour and motor performances of rheumatoid arthritis patients influenced by subclinical cognitive impairments? A clinical and neuroimaging study. Clin Exp Rheumatol 2002;20:491-497.

8. Arkema EV, Grunewald J, Kullberg S, et al. Sarcoidosis incidence and prevalence: a nationwide registerbased assessment in Sweden. Eur Respir J 2016;48:1690-1699.

9. Thomas KW, Hunninghake GW. Sarcoidosis. JAMA 2003;289:3300-3303.

10. Statement on sarcoidosis. Joint Statement of the American Thoracic Society (ATS), the European Respiratory Society (ERS) and the World Association of Sarcoidosis and Other Granulomatous Disorders (WASOG) adopted by the ATS Board of Directors and by the ERS Executive Committee, February 1999. Am J Respir Crit Care Med 1999;160:736-755.

11. Drent M, Lower EE, De Vries J. Sarcoidosis-associated fatigue. Eur Respir J 2012;40:255-263.

12. Fritz $D$, Voortman $M$, van de Beek $D$, et al. Many faces of neurosarcoidosis: from chronic meningitis to myelopathy. Curr Opin Pulm Med 2017;23 (5):439-446.

13. Leonhard SE, Fritz D, Eftimov F, et al. Neurosarcoidosis in a tertiary referral center: a cross-sectional cohort study. Medicine (Baltimore) 2016;95:e3277.

14. Drent M, Strookappe B, Hoitsma E, De Vries J. Consequences of sarcoidosis. Clin Chest Med 2015; 36:727-737.

15. Marcellis RG, Lenssen AF, Elfferich MD, et al. Exercise capacity, muscle strength and fatigue in sarcoidosis. Eur Respir J 2011;38:628-634.

16. Bakkers M, Merkies IS, Lauria G, et al. Intraepidermal nerve fiber density and its application in sarcoidosis. Neurology 2009;73:1142-1148.

17. Hoitsma $E$, Drent $M$, Verstraete $E$, et al. Abnormal warm and cold sensation thresholds suggestive of small-fibre neuropathy in sarcoidosis. Clin Neurophysiol 2003;114:2326-2333.

18. Zajicek JP, Scolding NJ, Foster O, et al. Central nervous system sarcoidosis-diagnosis and management. QJM 1999;92:103-117.

19. Marangoni S, Argentiero V, Tavolato B. Neurosarcoidosis. Clinical description of 7 cases with a proposal for a new diagnostic strategy. J Neurol 2006;253:488-495.

20. Voortman M, Fritz D, Vogels OJM, et al. Small fiber neuropathy: a disabling and underrecognized syndrome. Curr Opin Pulm Med 2017;23:447-457.

21. Tavee J, Culver D. Sarcoidosis and small-fiber neuropathy. Curr Pain Headache Rep 2011;15:201-206.

22. Datema M, Tannemaat MR, Drent M, Hoitsma E. [Neurosarcoidosis and paraneurosarcoidosis: new online registration of patients]. Ned Tijdschr Geneeskd 2015;159:A8383.

23. Culver DA, Ribeiro Neto ML, Moss BP, Willis MA. Neurosarcoidosis. Semin Respir Crit Care Med 2017; 38:499-513.

24. Hoitsma E, De Vries J, Drent M. The small fiber neuropathy screening list: construction and crossvalidation in sarcoidosis. Respir Med 2011;105:95-100. 
25. De Vries J, Michielsen H, Van Heck GL, Drent $M$. Measuring fatigue in sarcoidosis: the Fatigue Assessment Scale (FAS). Br J Health Psychol 2004;9:279-291.

26. Beste C, Kneiphof J, Woitalla D. Effects of fatigue on cognitive control in neurosarcoidosis. Eur Neuropsychopharmacol 2015;25:522-530.

27. van der Linden D, Frese M, Meijman TF. Mental fatigue and the control of cognitive processes: effects on perseveration and planning. Acta Psychol (Amst) 2003;113:45-65.

28. Dhillon HM, Tannock IF, Pond GR, et al. Perceived cognitive impairment in people with colorectal cancer who do and do not receive chemotherapy. J Cancer Surviv 2018;12(2):78-185.

29. Vardy JL, Stouten-Kemperman MM, Pond G, et al. A mechanistic cohort study evaluating cognitive impairment in women treated for breast cancer. Brain Imaging Behav 2017;13(1):15-26.

30. Tanaka M IA, Watanabe Y. Effects of mental fatigue on brain activity and cognitive performance: a magnetoencephalography study. Anat Physiol 2015;S4-002.

31. Moriarty O, Ruane N, O'Gorman D, et al. Cognitive impairment in patients with chronic neuropathic or radicular pain: an interaction of pain and age. Front Behav Neurosci 2017; 1:100.

32. Berryman C, Stanton TR, Jane Bowering K, et al. Evidence for working memory deficits in chronic pain: a systematic review and meta-analysis. Pain 2013;154:1181-1196.

33. Hendriks C, Drent $M$, de Kleijn $W$, et al. Everyday cognitive failure and depressive symptoms predict fatigue in sarcoidosis: a prospective follow-up study. Respiratory medicine 2018;138S:S24-30.

34. Bosse-Henck A, Koch R, Wirtz H, Hinz A. Fatigue and excessive daytime sleepiness in sarcoidosis: prevalence, predictors, and relationships between the two symptoms. Respiration 2017;94:186-197.

35. Drent $\mathrm{M}$, Wirnsberger RM, Breteler $\mathrm{MH}$, et al. Quality of life and depressive symptoms in patients suffering from sarcoidosis. Sarcoidosis Vasc Diffuse Lung Dis 1998;15:59-66.

36. Bosse-Henck A, Wirtz H, Hinz A. Subjective sleep quality in sarcoidosis. Sleep Med 2015;16:570-576.

37. Gerke AK, Hunninghake G. The immunology of sarcoidosis. Clin Chest Med 2008;29:379-390, vii.

38. Baune BT, Wiede F, Braun A, et al. Cognitive dysfunction in mice deficient for TNF- and its receptors. Am J Med Genet B Neuropsychiatr Genet 2008;147B:1056-1064.

39. McAfoose J, Baune BT. Evidence for a cytokine model of cognitive function. Neurosci Biobehav Rev 2009;33:355-366. 



\section{Chapter 5}

Management of neurosarcoidosis:

a clinical challenge

M. Voortman, M. Drent, R.P. Baughman Current Opinion of Neurology 2019;32(3):475-483 


\section{Abstract}

\section{Purpose of review}

Sarcoidosis is a complex disease with many faces, and the clinical manifestation and course of neurosarcoidosis are particularly variable. Although neurosarcoidosis occurs in up to ten percent of sarcoidosis patients, it can lead to significant morbidity and some mortality.

\section{Recent findings}

Three criteria are usually required for a diagnosis of (neuro)sarcoidosis: clinical and radiologic manifestations, noncaseating granulomas, and no evidence of alternative disease. Recent guidelines have helped to clarify criteria for diagnosing neurosarcoidosis. No firm guidelines exist on whether, when, and how treatment should be started. Treatment depends on the presentation and distribution, extensiveness and severity of neurosarcoidosis. As regards evidence-based treatment, only a few randomized controlled trials have been done. Hence, several aspects of (neuro)sarcoidosis management are not fully addressed by the current literature.

\section{Summary}

Significant advances have been made in the potential and accuracy of diagnostics for neurosarcoidosis. Treatment should be approached within the context of the patient's anticipated clinical course, avoidance of adverse drug effects, and, if necessary, from the perspective of the comprehensive management of a chronic disease. A multidisciplinary approach to the management of sarcoidosis is strongly recommended. 


\section{Introduction}

Sarcoidosis is a multisystem inflammatory disorder characterized by the formation of non-caseating granulomas in various organ systems, mainly the lungs and the lymphatic system. Although the pathogenesis of sarcoidosis has not been fully elucidated, environmental and genetic factors may contribute substantially to its pathogenesis, leading to an exaggerated granulomatous reaction. Sarcoidosis occurs throughout the world, affecting all races and ages. ${ }^{1}$ The clinical manifestation, natural history, and prognosis of sarcoidosis are highly variable. It can occur at almost any site in the body and most patients report disabling impairments, especially those with chronic disease. ${ }^{2}$ Neurosarcoidosis (NS) occurs in $5-10 \%$ of patients with sarcoidosis, rates which are not influenced by race or gender. ${ }^{3-5}$ The clinical manifestations of NS are also heterogeneous, as granulomas can affect any part of the nervous system, such as the meninges, brain, cranial nerves, spinal cord and peripheral nerves. ${ }^{6,7 * *}$ Cranial neuropathy is the most common manifestation, with facial nerves being most commonly affected, and is seen in $50 \%-70 \%$ of cases of NS. . $^{6 * *}$

The epidemiological assessment of sarcoidosis and its manifestations is problematic, due to the lack of consistent case definitions, lack of sensitivity and specificity of diagnostic tests, variable diagnostic intensity and variable diagnostic methods. Here we discuss the diagnostic approach and treatment of neurosarcoidosis.

\section{Diagnostic approach to neurosarcoidosis}

Although the diagnosis of sarcoidosis is a complex procedure and can be difficult for clinicians, the potential and accuracy of diagnostics for sarcoidosis, especially to assess organ involvement, have been improved in recent decades. However, there is still no single diagnostic test for sarcoidosis. The finding of granuloma in a single organ is not specific for this disease, since many other conditions can cause granulomas. According to the joint statement of the American Thoracic Society, the European Respiratory Society, and the World Association of Sarcoidosis and Other Granulomatous Disorders (WASOG), three criteria are usually required for a diagnosis of sarcoidosis: typical clinical and radiologic manifestations, non-caseating granulomas, and no evidence of alternative disease. ${ }^{8}$ In case of suspected sarcoidosis, the diagnostic procedures aim to accomplish the following goals: (1) provide histological confirmation of the disease; (2) evaluate the extent and severity of organ involvement; (3) assess whether the disease is stable or likely to progress; and (4) determine if the patient will benefit from treatment. 
The presence of specific clinical features, especially multi-organ involvement, can enhance the diagnostic certainty. The WASOG recently developed criteria for categorizing sarcoidosis organ involvement as "highly probable", "at least probable", or "possible." The WASOG instrument provides a structured system for identifying the clinico-radiologic findings in patients with sarcoidosis and may standardize organ involvement reporting for patients with sarcoidosis. ${ }^{9}$ Bickett et al. developed the Sarcoidosis Diagnostic Score (SDS) to summarize the clinical features of patients with possible sarcoidosis. ${ }^{10}$ For each organ, it allocates points if the organ has a positive biopsy ( 5 points), if one or more features are consistent with highly probable organ involvement ( 3 points), or at least probable organ involvement (2 points). The resulting SDS was found to have high sensitivity and specificity. ${ }^{10}$ This score can be used to support the clinical diagnosis of sarcoidosis referred to in Figure 5.1. Confirmation by other studies may establish the value of this scoring system.

Patients with suspected NS require a careful assessment of the systemic manifestations and neurologic evaluation. Since sarcoidosis is a multi-organ disease, a diagnosis of neurosarcoidosis does not rest merely on neurologic features. Table 5.1 lists the routine testing recommended for suspected NS patients as part of their initial evaluation; chest imaging is part of this evaluation. The chest radiographic finding of bilateral hilar adenopathy with right paratracheal involvement makes sarcoidosis highly probable. ${ }^{11,12}$ Recently, it was shown that high resolution computed tomography (HRCT) was superior, and therefore preferable, to chest radiography for evaluating the features, pattern and distribution of the parenchymal lesions and mediastinal lymph nodes, as well as for assessing the stage and activity of the disease and aiding the detection of subtle parenchymal lesions which are liable to be missed on conventional imaging. ${ }^{13}$ Characteristic findings are nodular infiltrates with bronchovascular and subpleural distribution, thickened interlobular septa, architectural distortion, and conglomerate masses originating from the coalescence of nodules in the perihilar, peribronchovascular, or subpleural regions. ${ }^{14}$ FDG PET/CT (fluorodeoxyglucose positron emission tomography/computed tomography) can be useful in cases in which other imaging modalities have been unsuccessful in detecting sarcoidosis activity. FDG PET-CT is mainly used for the detection of extraneural localizations and the identification of extraneural biopsy sites. ${ }^{15-19}$ The brain can be included in whole body PET/CT examinations with only marginal increases in examination time and radiation dose (due to the extended field of low-dose CT scanning). ${ }^{20}$ The intense physiologic uptake in the brain limits accurate evaluation of brain lesions. However, the FDG PET findings may provide the first suggestion of a diagnosis of sarcoidosis involving only neurologic symptoms. 
Depending on the part of the brain involved, a sarcoidosis lesion can be hypo- or hypermetabolic. In contrast, spinal cord lesions appear as hypermetabolic because the basal metabolism of the spinal cord is one-third that of the cerebral gray matter. Its capability to depict metabolic changes also enables FDG PET to be used to monitor therapy before morphologic changes are detected with conventional imaging. ${ }^{21}$ Novel PET strategies, in order to overcome the limitations of FDG, have recently been introduced, including imaging of somatostatin receptors or C-X-C motif chemokine receptor (CXCR4), which are overexpressed on the cell surface of activated macrophages. ${ }^{22,23}$ Given the specific nature of their signal, these tracers might be used in cardiac and neurosarcoidosis, since these organs have an intense physiologic uptake of FDG. It might thereby be possible to directly assess the extent of inflammatory activity, localize sites of activity, and monitor response to therapy. Further studies are needed to evaluate this new PET strategy for NS.

Table 5.1 Recommended tests for initial evaluation of sarcoidosis.

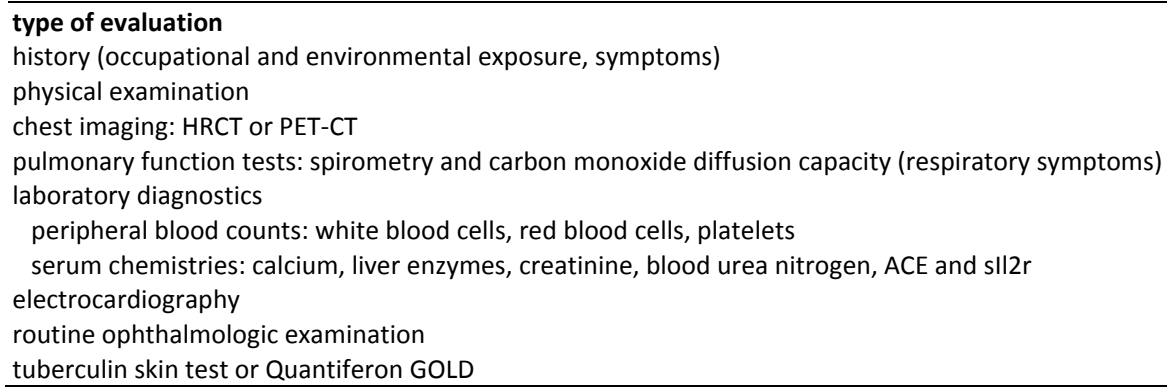

$\mathrm{HRCT}=$ high resolution computer tomography; $\mathrm{PET}-\mathrm{CT}=$ positron emission tomography-computer tomography; $\mathrm{ACE}=$ angiotensin converting enzyme; sIL2r=soluble interleukin 2 receptor.

In patients for whom extra-neural tissue is needed to identify granulomas, bronchoscopy can provide multiple options for diagnosing sarcoidosis. These include bronchoalveolar lavage (BAL), endobronchial biopsy, transbronchial lung biopsy (TBLB), cryobiopsy, and endobronchial ultrasound fine-needle aspiration (EBUS-FNA). These tests are complementary and the combination of tests is superior to performing any single test. ${ }^{24,25}$ While bronchoscopy is often performed in patients with pulmonary disease, a recent study among patients presenting with manifestations of suspected cardiac sarcoidosis without a prior history of lung disease demonstrated that lung and mediastinal lymph node biopsies confirmed extracardiac sarcoidosis in $58 \%$ of patients, and BAL cellular analyses were suggestive of extracardiac sarcoidosis in $67 \%$ of patients, even those without abnormalities on preliminary $\mathrm{HRCT}^{26}$ Hence, the use of 
bronchoscopy in patients with extra-pulmonary disease, even with normal CT scans, can be helpful in diagnosing sarcoidosis. ${ }^{26,27}$

Diagnostic criteria for neurologic involvement in sarcoidosis patients have been developed by neurologists and general sarcoidosis experts. ${ }^{7^{* *}, 9}$ The Neurosarcoidosis Consortium Consensus Group, an expert panel of physicians experienced in the management of patients with sarcoidosis and/or neurosarcoidosis, engaged in an iterative process to define NS, and developed a practical clinical diagnostics approach for patients with suspected NS. The resulting consensus clinical definition of NS aimed to enhance the clinical care of patients with suspected NS and to encourage standardization of research initiatives to address this disease manifestation. The authors identified criteria indicating possible, probable or definite neurologic involvement (see Table 5.2).

Table 5.2 Diagnostic criteria for neurosarcoidosis (central and peripheral nervous system involvement). ${ }^{7}$

possible

1. the clinical presentation and diagnostic evaluation suggest neurosarcoidosis, as defined by the clinical manifestations and MRI, CSF and/or EMG/NCS findings typical of granulomatous inflammation of the nervous system and after rigorous exclusion of other causes.

2. there is no pathological confirmation of granulomatous disease.

probable

1. the clinical presentation and diagnostic evaluation suggest neurosarcoidosis, as defined by the clinical manifestations and MRI, CSF and/or EMG/NCS findings typical of granulomatous inflammation of the nervous system and after rigorous exclusion of other causes.

2. there is pathological confirmation of systemic granulomatous disease consistent with sarcoidosis.

definite

1. the clinical presentation and diagnostic evaluation suggest neurosarcoidosis, as defined by the clinical manifestations, MRI, CSF and/or EMG/NCS findings typical of granulomatous inflammation of the nervous system, after rigorous exclusion of other causes.

2. the nervous system abnormality is consistent with neurosarcoidosis.

type a. Extraneural sarcoidosis is evident

type b. No extraneural sarcoidosis is evident (isolated neurosarcoidosis).

$\mathrm{MRI}=$ magnetic resonance imaging; $\mathrm{CSF}=$ cerebrospinal fluid; $\mathrm{EMG}=$ electromyography; $\mathrm{NCS}=$ nerve conduction studies.

Gadolinium-enhanced MRI is the modality of choice for the diagnosis of NS. The abnormalities seen at MRI include periventricular white matter lesions, meningitis or meningoencephalitis, solid parenchymal enhancing lesions, cranial neuritis and myelopathy. ${ }^{28,29}$ Since bone sarcoidosis often involves the spine, patients undergoing MRI scanning for back pain or radiculopathy may be found to have an infiltrative lesion. ${ }^{30}$ Such an MRI pattern is often confused with malignancy and in some cases a bone biopsy may be required to confirm sarcoidosis. ${ }^{31}$ 


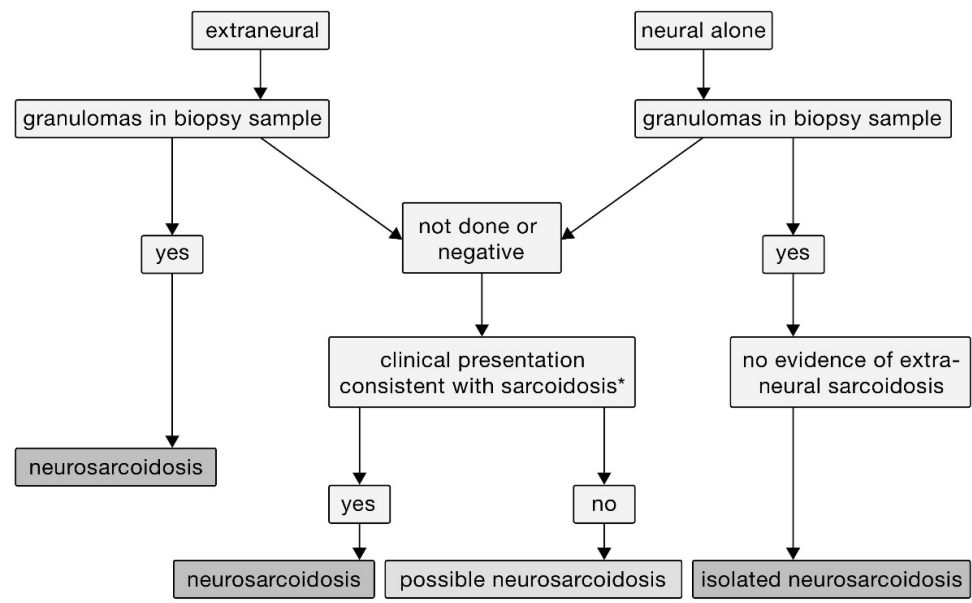

Figure 5.1 Approach to diagnosis of neurosarcoidosis.

Figure 5.1 illustrates the approach to diagnosing NS. In Table 5.3 the various manifestations of neurosarcoidosis including myelitis (see Figure 5.2) are summarized. For a patient with neurologic signs and symptoms consistent with NS, the next step is to check for extra-neural disease. If there is evidence of such disease based on a biopsy showing granuloma, the patient most likely has NS. If the patient has not had a biopsy or the biopsy was negative for granuloma, the extra-neural features are then evaluated. If the patient's clinical presentation is consistent with sarcoidosis and other causes have been excluded, the patient is assumed to have NS. If there are insufficient clinical features to support the diagnosis, the patient is said to have possible NS. Isolated NS is diagnosed in a patient who presents with only neural symptoms, a biopsy which demonstrates granulomas, and no clinical features of extra-neural sarcoidosis. Patients who present with neurologic disease should always be evaluated for extra-neural sarcoidosis. 
Table 5.3 Summary of various neurosarcoidosis manifestations. ${ }^{7^{* *}, 32,33}$

\begin{tabular}{|c|c|c|}
\hline Neurosarcoidosis manifestation & Prevalence & Comments \\
\hline cranial nerve palsy & $31-55 \%$ & $\begin{array}{l}\text { facial and optic nerves are the most commonly affected; } \\
\text { uni- or bilateral involvement }\end{array}$ \\
\hline chronic aseptic meningitis & $16-37 \%$ & $\begin{array}{l}\text { subacute or chronic lymphocytic meningitis; dural } \\
\text { involvement including pachymeningitis, dural mass } \\
\text { mimicking meningioma }\end{array}$ \\
\hline spinal cord disease / myelitis & $18-23 \%$ & $\begin{array}{l}\text { subpial intramedullary lesions, typically longitudinally- } \\
\text { extensive; myelitis predilection cervicothoracic }\end{array}$ \\
\hline cerebral parenchymal disease & $21 \%$ & $\begin{array}{l}\text { small cortical or periventricular white matter lesions; } \\
\text { mimicking MS or micro-ischemic lesions, larger solitary } \\
\text { aggregates of granulomas can masquerade as neoplasms. }\end{array}$ \\
\hline $\begin{array}{l}\text { neuro-endocrine (hypothalamo- } \\
\text { pituitary) involvement }\end{array}$ & $6-9 \%$ & $\begin{array}{l}\text { hormonal disturbances including hypothyreoidism, } \\
\text { hypogonadism, panhypopituitarism, syndrome of } \\
\text { inappropriate antidiuretic hormone (SIADH) }\end{array}$ \\
\hline hydrocephalus & $9-10 \%$ & $\begin{array}{l}\text { communicating and non-communicating hydrocephalus; } \\
\text { combination with leptomeningeal enhancement along the } \\
\text { skull base }\end{array}$ \\
\hline cerebral infarction & $6 \%$ & $\begin{array}{l}\text { stroke can be due to in situ thrombosis, compression of a } \\
\text { large vessel by a granulomatous mass, sinovenous } \\
\text { thrombosis and intracerebral haemorrhage }\end{array}$ \\
\hline peripheral nervous system & $17 \%$ & $\begin{array}{l}\text { large fiber involvement: most commonly axonal distal } \\
\text { sensorimotor polyneuropathy or asymmetric } \\
\text { polyradiculoneuropathy (non-length dependent } \\
\text { distribution) }\end{array}$ \\
\hline
\end{tabular}

MS=multiple sclerosis

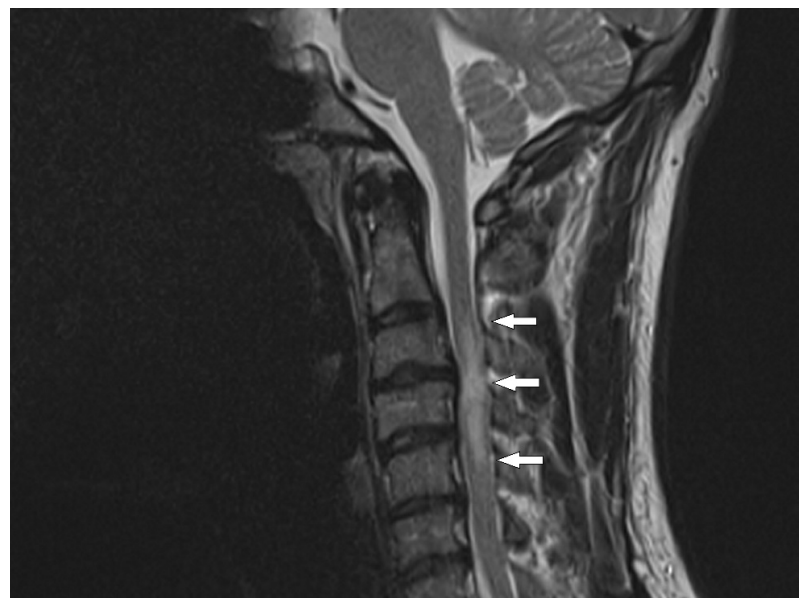

Figure 5.2 36-year-old male, presenting with numbness in both arms and cervical pain. MRI showed a cervical myelitis (C3-C5, arrows). Bilateral mediastinal lymphadenopathy was seen on a chest Xray and the diagnosis sarcoidosis was confirmed with an endobronchial ultrasound fine-needle aspiration (EBUS-FNA). 


\section{Non-organ-related sarcoidosis-associated symptoms}

There are several consequences of sarcoidosis, including fatigue, cognitive failure and small fiber neuropathy (SFN), which are not directly related to granulomatous involvement. $^{2,34}$ Sarcoidosis-associated fatigue is reported in more than half of patients. $^{35,36}$ It is commonly associated with other non-organ-related problems, including SFN and cognitive failure. ${ }^{37-39^{*}}$ Questionnaires can be helpful in objectifying these complaints. ${ }^{40-42} \mathrm{~A}$ multidisciplinary approach including psychological counselling is recommended. ${ }^{43,44}$

SFN, which is found in many conditions, ${ }^{45,46^{*}}$ was originally described for sarcoidosis by Hoitsma et al. in 2002. ${ }^{47}$ There is no diagnostic gold standard for SFN. Nerve conduction studies (NCS) are performed as the first diagnostic test to exclude large-fiber disorders. Skin biopsies with quantitative counting of the nerve fibers have been proposed. The fewer the nerve fibers, the greater the likelihood of SFN. ${ }^{45}$ However, the disease can be patchy, leading to sampling errors and false negative findings. The disease is also invasive.

An SFN screening tool has been developed for patients with sarcoidosis, called the SFN Screening List (SFNSL). ${ }^{42}$ This screening tool can distinguish between patients who are very likely to have SFN-associated symptoms and those without such symptoms. A substantial percentage of patients have intermediate scores (between highly likely and unlikely SFN), and these patients require additional tests to confirm the diagnosis of SFN. Despite its limitations, the SFNSL has proved useful in assessing response to therapy. $^{48}$

An interesting new test for SFN is corneal confocal microscopy. Photographs are taken of the cornea and a quantitative assessment of nerve fibers is made. A correlation has been established between the severity of neuropathy and progressive corneal nerve degeneration. ${ }^{49,50}$ Unfortunately, this technique is not widely available, and has not yet been validated against other measures of SFN. The changes demonstrated during treatment for SFN did not correlate with other markers of SFN. ${ }^{51}$

\section{Treatment of neurosarcoidosis}

Treatment is almost always warranted in NS. Treatment strategies are mainly based on expert opinion and small retrospective studies. So far, no randomized controlled trials have been performed. ${ }^{6}$ The intensity of treatment depends on the severity of the NS manifestations. For example, a facial nerve paralysis can most often be treated with only a few weeks of prednisone monotherapy, and rarely recurs, ${ }^{52}$ while in the case of 
spinal cord disease a second-line (methotrexate [MTX], azathioprine, mycophenolate mofetil [MMF]) or third-line (tumour necrosis factor [TNF]- $\alpha$ inhibitors; infliximab or adalimumab) agent is initiated earlier on in the treatment. ${ }^{53,54}$ Limited data are available on the optimal treatment options for NS.

Treatment of sarcoidosis has commonly been empirical. However, an increasing number of studies have been published which provide a level of confidence regarding general therapeutic recommendations..$^{55^{* *}}$ These recommendations have to be modified for NS. Figure 5.3 shows a proposed stepwise approach to the treatment of NS other than that involving the seventh cranial nerve alone.

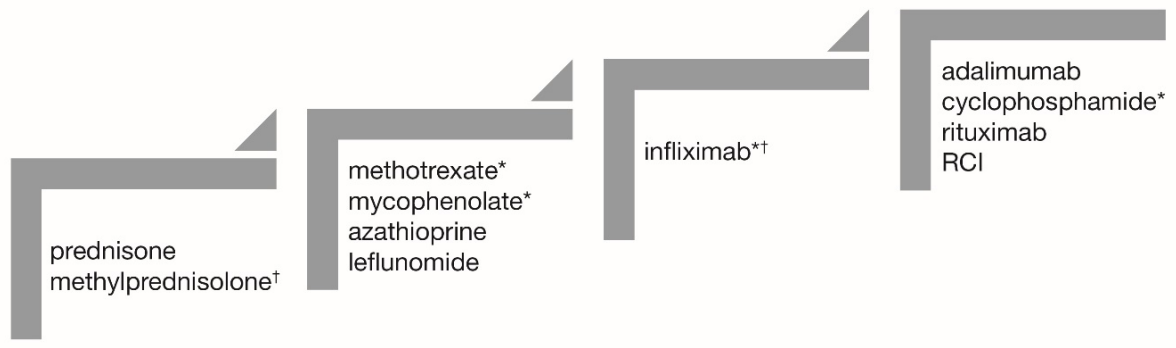

Figure 5.3 Proposed stepwise treatment for neurosarcoidosis. $\mathrm{RCl}=$ repository corticotropin injection.

*indicates drugs that have been reported specifically for neurosarcoidosis. tindicates therapies which may be used for severe or progressive disease. See text for further details.

Initial therapy consists of glucocorticoids, usually oral prednisone. For a patient with severe disease or disease progressing despite oral therapy, high-dose intravenous methylprednisolone may be useful. High-dose oral prednisone has been shown to be as effective as high dose intravenous steroids. ${ }^{56}$ Most of the prednisone recommendations are based on retrospective studies, and the dosage of prednisone varies. Failure of steroid monotherapy is common, due to severity of the disease or because of the toxicity of the high-dose prednisone used in NS. In one study over $80 \%$ of patients went on to second- and third-line therapy. ${ }^{52}$

The next step involves a steroid-sparing or second-line agent. MTX has been the most widely used steroid-sparing agent for sarcoidosis. ${ }^{57}$ In one of the few case series examining the use of MTX specifically for NS, the response rate was $63 \%{ }^{52}$ More recently, NS patients treated with MTX were successfully weaned down to prednisone in half of cases and maintained on that regimen for years. ${ }^{53}$ Mycophenolate, another steroid-sparing second-line agent, has a different toxicity profile than methotrexate and may be more rapidly effective in treating NS. ${ }^{58}$ However, in one study, mycophenolate 
treatment was associated with a significantly higher relapse rate when prednisone was withdrawn. ${ }^{53}$

The use of two other cytotoxic agents, azathioprine and leflunomide, is based on studies of non-NS patients. In one large study, azathioprine was as effective as methotrexate but associated with some more toxicity. ${ }^{59}$ Leflunomide has been reported to be an effective alternative to methotrexate with a different toxicity profile. ${ }^{60,61}$ However, leflunomide can cause a peripheral neuropathy, which limits its use in NS. ${ }^{62}$

The next step is the use of TNF- $\alpha$ inhibitors. Although not all TNF- $\alpha$ inhibitors have been successful in sarcoidosis, the monoclonal anti-TNF- $\alpha$ antibody infliximab was found to be superior to placebo in treating advanced pulmonary sarcoidosis. ${ }^{63,64}$ A Delphi study amongst the world's leading sarcoidologists resulted in practical recommendations for the use of TNF- $\alpha$ inhibitors in sarcoidosis, to support clinicians in the management of patients with refractory sarcoidosis. ${ }^{65}$ Based on expert experience and recent studies, infliximab is now considered the main third-line treatment option in sarcoidosis. Two recent papers reported on relatively large studies of infliximab for NS in the United States and France. ${ }^{66^{*}, 67}$ In both of these series, response to treatment was seen even when other therapies had failed. Unfortunately, infections and other toxicities were encountered in a significant number of cases, ${ }^{66^{*}}$ although these were similar to those for second-line treatment options. ${ }^{59}$ Treatment withdrawal was associated with a high rate of relapse of the disease. ${ }^{66^{*}, 67}$ Treatment with infliximab is expensive, creating a barrier limiting universal access to this effective therapeutic agent. Recently, biosimilars of infliximab have become available. In view of the working mechanism of the original biological and that of the biosimilars, it is highly likely that the therapeutic effect of both agents is comparable. Hence, inclusion of biosimilars in the treatment regime could lower the costs of TNF- $\alpha$ inhibitors in sarcoidosis. ${ }^{68}$ The infliximab biosimilar inflectra ${ }^{\circledR}$ proved to be effective in the treatment of refractory sarcoidosis, with a safety profile comparable to that of the reference product infliximab. ${ }^{69,70^{*}}$

For patients for whom infliximab fails due to intolerance or lack of efficacy, or for whom TNF- $\alpha$ inhibitors are contra-indicated, several other potential agents have been reported. Adalimumab is usually reserved for patients who develop reactions to infliximab, and the drug has been shown to be effective in that situation. ${ }^{71}$ Intravenous cyclophosphamide was reported to be effective in the treatment of refractory NS, although less so than infliximab..$^{52,72-74}$ It may still be an alternative for refractory NS patients. For patients for whom TNF- $\alpha$ inhibitors are contra-indicated, such as those with malignancy or advanced congestive heart failure, rituximab has also been reported to be useful. ${ }^{75,76}$ 


\section{Treatment of sarcoidosis-associated small fiber neuropathy}

Currently, there is no cure for SFN, and only symptomatic relief of complaints is achieved. ${ }^{46^{*}}$ Unfortunately, symptomatic treatment for neuropathic pain often provides only partial relief from pain, without effects on autonomic dysfunction, and is often associated with (sometimes severe) side effects. Some data suggest some effectiveness of immunoglobulins and TNF- $\alpha$ inhibitors. ${ }^{38,77,78^{*}}$ Whether these expensive treatments should be initiated as a causative treatment for SFN is unclear and is currently being investigated. Cibinetide ${ }^{\circledR}$ (ARA290) seems a promising new drug to relieve pain and increase corneal and skin nerve fiber density in sarcoidosis-associated SFN. ${ }^{48,79,80}$ However, a recent placebo-controlled trial demonstrated changes in corneal nerve fibers, but not in symptoms. ${ }^{51}$ Further studies, including a wider dose range, are needed to clarify the role of this drug.

\section{Conclusion}

As sarcoidosis is a multi-organ disorder that imposes a burden on patients' lives, patients may initially present to various organ specialists, depending on the presenting symptoms. Therefore, a multidisciplinary approach is recommended for the management of sarcoidosis, with considerable patient participation focusing on somatic as well as psychosocial aspects of this erratic disorder. Neurosarcoidosis can be extreme burdensome for patients and their families. Recent reports have helped to clarify a diagnostic strategy for this disease entity. Treatment options, especially with infliximab or biosimilars, may prove an effective way to control the disease. 


\section{References}

Papers of particular interest, published within the annual period of review, have been highlighted as:

* of special interest

** of outstanding interest

1 Valeyre D, Prasse A, Nunes H, et al. Sarcoidosis. Lancet 2014; 383:1155-1167.

2 Drent M, Strookappe B, Hoitsma E, De Vries J. Consequences of sarcoidosis. Clin Chest Med 2015; 36:727-737.

3 Baughman RP, Teirstein AS, Judson MA, et al. Clinical characteristics of patients in a case control study of sarcoidosis. Am J Respir Crit Care Med 2001;164:1885-1889.

4 Judson MA, Boan AD, Lackland DT. The clinical course of sarcoidosis: presentation, diagnosis, and treatment in a large white and black cohort in the United States. Sarcoidosis Vasc Diffuse Lung Dis 2012; 29:119-127.

5 Pietinalho A, Ohmichi M, Hiraga $Y$, et al. The mode of presentation of sarcoidosis in Finland and Hokkaido, Japan. A comparative analysis of 571 Finnish and 686 Japanese patients. Sarcoidosis Vasc Diffuse Lung Dis 1996;13:159-166.

6 Fritz D, Voortman M, van de Beek D, et al. Many faces of neurosarcoidosis: from chronic meningitis to myelopathy. Curr Opin Pulm Med 2017;23:439-446.

7 Stern BJ, Royal W, 3rd, Gelfand JM, et al. Definition and consensus diagnostic criteria for neurosarcoidosis: from the neurosarcoidosis consortium consensus group. JAMA Neurol 2018;75(12): 1546-1553.

**The Neurosarcoidosis Consortium Consensus Group, an expert panel of physicians experienced in the management of patients with sarcoidosis and/or neurosarcoidosis, engaged in an iterative process to define neurosarcoidosis and developed a practical clinical diagnostic approach to patients with suspected neurosarcoidosis.

8 Hunninghake GW, Costabel U, Ando M, et al. ATS/ERS/WASOG statement on sarcoidosis. American Thoracic Society/European Respiratory Society/World Association of Sarcoidosis and other Granulomatous Disorders. Sarcoidosis Vasc Diffuse Lung Dis 1999;16:149-173.

9 Judson MA, Costabel U, Drent $M$, et al. The WASOG sarcoidosis organ assessment instrument: an update of a previous clinical tool. Sarcoidosis Vasc Diffuse Lung Dis 2014;31:19-27.

10 Bickett AN, Lower EE, Baughman RP. Sarcoidosis diagnostic score: a systematic evaluation to enhance the diagnosis of sarcoidosis. Chest 2018;154:1052-1060.

11 Winterbauer RH, Belic N, Moores KD. Clinical interpretation of bilateral hilar adenopathy. Ann Intern Med 1973;78:65-71.

12 Reich JM, Brouns MC, O'Connor EA, Edwards MJ. Mediastinoscopy in patients with presumptive stage I sarcoidosis: a risk/benefit, cost/benefit analysis. Chest 1998;113:147-153.

13 Dhagat PK, Singh S, Jain M, et al. Thoracic sarcoidosis: imaging with high resolution computed tomography. J Clin Diagn Res 2017;11:TC15-TC18.

14 Drent M, De Vries J, Lenters M, et al. Sarcoidosis: assessment of disease severity using HRCT. Eur Radiol 2003;13:2462-2471.

15 Teirstein AS, Machac J, Almeida O, et al. Results of 188 whole-body fluorodeoxyglucose positron emission tomography scans in 137 patients with sarcoidosis. Chest 2007;132:1949-1953.

16 Sakushima K, Yabe I, Shiga T, et al. FDG-PET SUV can distinguish between spinal sarcoidosis and myelopathy with canal stenosis. J Neurol 2011;258:227-230.

17 Mostard RL, van Kroonenburgh MJ, Drent M. The role of the PET scan in the management of sarcoidosis. Curr Opin Pulm Med 2013;19:538-544.

18 Keijsers RG, Grutters JC, Thomeer M, et al. Imaging the inflammatory activity of sarcoidosis: sensitivity and inter observer agreement of (67)Ga imaging and (18)F-FDG PET. Q J Nucl Med Mol Imaging 2011; 55:66-71.

19 Akaike G, Itani $\mathrm{M}$, Shah $\mathrm{H}$, et al. PET/CT in the diagnosis and workup of sarcoidosis: focus on atypical manifestations. Radiographics 2018;38:1536-1549. 
20 Ganeshan D, Menias CO, Lubner MG, et al. Sarcoidosis from head to toe: what the radiologist needs to know. Radiographics 2018;38:1180-1200.

21 Vorselaars $\mathrm{AD}$, Verwoerd $\mathrm{A}$, van Moorsel $\mathrm{CH}$, et al. Prediction of relapse after discontinuation of infliximab therapy in severe sarcoidosis. Eur Respir J 2014;43:602-609.

22 Armani C, Catalani E, Balbarini A, et al. Expression, pharmacology, and functional role of somatostatin receptor subtypes 1 and 2 in human macrophages. J Leukoc Biol 2007;81:845-855.

23 Kircher M, Lapa C. Novel noninvasive nuclear medicine imaging techniques for cardiac inflammation. Curr Cardiovasc Imaging Rep 2017;10:6.

$24 \mathrm{Hu}$ LX, Chen RX, Huang $\mathrm{H}$, et al. Endobronchial ultrasound-guided transbronchial needle aspiration versus standard bronchoscopic modalities for diagnosis of sarcoidosis: a meta-analysis. Chin Med J (Engl) 2016;129:1607-1615.

25 Aragaki-Nakahodo AA, Baughman RP, Shipley RT, Benzaquen S. The complimentary role of transbronchial lung cryobiopsy and endobronchial ultrasound fine needle aspiration in the diagnosis of sarcoidosis. Respir Med 2017;131:65-69.

26 Petek BJ, Rosenthal DG, Patton KK, et al. Cardiac sarcoidosis: diagnosis confirmation by bronchoalveolar lavage and lung biopsy. Respir Med 2018;144S:S13-S19.

27 Takahashi T, Azuma A, Abe S, et al. Significance of lymphocytosis in bronchoalveolar lavage in suspected ocular sarcoidosis. Eur Respir J 2001;18:515-521.

28 Tavee JO, Stern BJ. Neurosarcoidosis. Continuum (Minneap Minn) 2014;20:545-559.

29 Bathla G, Singh AK, Policeni B, et al. Imaging of neurosarcoidosis: common, uncommon, and rare. Clin Radiol 2016;71:96-106.

30 Zhou Y, Lower EE, Li H, et al. Clinical characteristics of patients with bone sarcoidosis. Semin Arthritis Rheum 2017;47:143-148.

31 Moore SL, Teirstein A, Golimbu C. MRI of sarcoidosis patients with musculoskeletal symptoms. AJR Am J Roentgenol 2005;185:154-159.

32 Fritz D, van de Beek D, Brouwer MC. Clinical features, treatment and outcome in neurosarcoidosis: systematic review and meta-analysis. BMC Neurol 2016;16:220.

33 Leonhard SE, Fritz D, Eftimov F, et al. Neurosarcoidosis in a tertiary referral center: a cross-sectional cohort study. Medicine (Baltimore) 2016;95:e3277.

34 Hoitsma E, De Vries J, van Santen-Hoeufft M, et al. Impact of pain in a Dutch sarcoidosis patient population. Sarcoidosis Vasc Diffuse Lung Dis 2003;20:33-39.

35 Gvozdenovic BS, Mihailovic-Vucinic V, llic-Dudvarski A, et al. Differences in symptom severity and health status impairment between patients with pulmonary and pulmonary plus extrapulmonary sarcoidosis. Respir Med 2008;102:1636-1642.

36 Drent M, Lower EE, De Vries J. Sarcoidosis-associated fatigue. Eur Respir J 2012;40:255-263.

37 Hendriks C, Drent M, De Kleijn W, et al. Everyday cognitive failure and depressive symptoms predict fatigue in sarcoidosis: a prospective follow-up study. Respir Med 2018;138S:S24-S30.

38 Elfferich MD, Nelemans PJ, Ponds RW, et al. Everyday cognitive failure in sarcoidosis: the prevalence and the effect of anti-TNF-alpha treatment. Respiration 2010;80:212-219.

39 Voortman M, De Vries J, Hendriks CMR; et al. Everyday cognitive failure in patients suffering from neurosarcoidosis. Sarcoidosis Vasc Diffuse Lung Dis 2019;36;2-10.

* This study demonstrated the burden of cognitive failure in neurosarcoidosis.

40 Broadbent DE, Cooper PF, FitzGerald P, Parkes KR. The Cognitive Failures Questionnaire (CFQ) and its correlates. Br J Clin Psychol 1982; 21(Pt 1):1-16.

41 De Vries J, Michielsen H, Van Heck GL, Drent M. Measuring fatigue in sarcoidosis: the Fatigue Assessment Scale (FAS). Br J Health Psychol 2004;9:279-291.

42 Hoitsma E, De Vries J, Drent $M$. The small fiber neuropathy screening list: construction and crossvalidation in sarcoidosis. Respir Med 2011;105:95-100.

43 Drent M. Sarcoidosis: benefits of a multidisciplinary approach. Eur J Intern Med 2003;14:217-220.

44 Moor CC, van Manen MJG, van Hagen PM, et al. Needs, perceptions and education in sarcoidosis: a live interactive survey of patients and partners. Lung 2018;196:569-575.

45 Tavee J, Zhou L. Small fiber neuropathy: a burning problem. Cleve Clin J Med 2009;76:297-305. 
46 Voortman M, Fritz D, Vogels OJM, et al. Small fiber neuropathy: a disabling and underrecognized syndrome. Curr Opin Pulm Med 2017;23:447-457.

* This review underlines that SFN is difficult to diagnose, as a gold standard is still lacking. In the majority of cases, the only option is symptomatic treatment.

47 Hoitsma E, Marziniak M, Faber CG, et al. Small fibre neuropathy in sarcoidosis. Lancet 2002;359:20852086.

48 Heij L, Niesters M, Swartjes M, et al. Safety and efficacy of ARA 290 in sarcoidosis patients with symptoms of small fiber neuropathy: a randomized, double-blind pilot study. Mol Med 2012;18:14301436.

49 Stettner M, Hinrichs L, Guthoff R, et al. Corneal confocal microscopy in chronic inflammatory demyelinating polyneuropathy. Ann Clin Transl Neurol 2016;3:88-100.

50 Tavakoli M, Quattrini C, Abbott C, et al. Corneal confocal microscopy: a novel noninvasive test to diagnose and stratify the severity of human diabetic neuropathy. Diabetes Care 2010;33:1792-1797.

51 Brines M, Culver DA, Ferdousi M, et al. Corneal nerve fiber size adds utility to the diagnosis and assessment of therapeutic response in patients with small fiber neuropathy. Sci Rep 2018;8:4734.

52 Lower EE, Broderick JP, Brott TG, Baughman RP. Diagnosis and management of neurological sarcoidosis. Arch Intern Med 1997;157:1864-1868.

53 Bitoun S, Bouvry D, Borie R, et al. Treatment of neurosarcoidosis: a comparative study of methotrexate and mycophenolate mofetil. Neurology 2016;87:2517-2521.

54 Bradley DA, Lower EE, Baughman RP. Diagnosis and management of spinal cord sarcoidosis. Sarcoidosis Vasc Diffuse Lung Dis 2006;23:58-65.

55 James WE, Baughman R. Treatment of sarcoidosis: grading the evidence. Expert Rev Clin Pharmacol 2018;11:677-687.

** Excellent review discussing the current therapeutic options for sarcoidosis.

56 Ramo-Tello C, Grau-Lopez L, Tintore M, et al. A randomized clinical trial of oral versus intravenous methylprednisolone for relapse of MS. Mult Scler 2014;20:717-725.

57 Cremers JP, Drent M, Bast A, et al. Multinational evidence-based World Association of Sarcoidosis and Other Granulomatous Disorders recommendations for the use of methotrexate in sarcoidosis: integrating systematic literature research and expert opinion of sarcoidologists worldwide. Curr Opin Pulm Med 2013;19:545-561.

58 Androdias G, Maillet D, Marignier R, et al. Mycophenolate mofetil may be effective in CNS sarcoidosis but not in sarcoid myopathy. Neurology 2011;76:1168-1172.

59 Vorselaars ADM, Wuyts WA, Vorselaars VMM, et al. Methotrexate vs azathioprine in second-line therapy of sarcoidosis. Chest 2013;144:805-812.

60 Baughman RP, Lower EE. Leflunomide for chronic sarcoidosis. Sarcoidosis Vasc Diffuse Lung Dis 2004; 21:43-48.

61 Sahoo DH, Bandyopadhyay D, Xu M, et al. Effectiveness and safety of leflunomide for pulmonary and extrapulmonary sarcoidosis. Eur Respir J 2011;38:1145-1150.

62 Kho LK, Kermode AG. Leflunomide-induced peripheral neuropathy. J Clin Neurosci 2007;14:179-181.

63 Baughman RP, Drent M, Kavuru M, et al. Infliximab therapy in patients with chronic sarcoidosis and pulmonary involvement. Am J Respir Crit Care Med 2006;174:795-802.

64 Baughman RP, Lower EE. Infliximab for refractory sarcoidosis. Sarcoidosis Vasc Diffuse Lung Dis 2001; 18:70-74.

65 Drent M, Cremers JP, Jansen TL, Baughman RP. Practical eminence and experience-based recommendations for use of TNF-alpha inhibitors in sarcoidosis. Sarcoidosis Vasc Diffuse Lung Dis 2014; 31:91-107.

66 Cohen Aubart F, Bouvry D, Galanaud D, et al. Long-term outcomes of refractory neurosarcoidosis treated with infliximab. J Neurol 2017;264:891-897.

* A retrospective cohort study describing the long-term outcomes of treatment with infliximab in patients with biopsy-proven neurosarcoidosis. At 6 months after initiation of infliximab treatment, 33\% had complete remission and $56 \%$ partial remission, whereas stable disease was observed in $11 \%$. After a median follow-up of 20 months, $50 \%$ of patients had a relapse. Side-effects were observed in eight patients, seven of whom had infections. 
67 Gelfand JM, Bradshaw MJ, Stern BJ, et al. Infliximab for the treatment of CNS sarcoidosis: a multiinstitutional series. Neurology 2017;89:2092-2100.

68 Veltkamp M, Drent M, Baughman RP. Infliximab or biosimilars in sarcoidosis; to switch or not to switch? Sarcoidosis Vasc Diffuse Lung Dis 2016;32:280-283.

69 Merinopoulos D, Hayes F, Gallagher DA, Dasgupta B. A case report of neurosarcoidosis successfully treated with an infliximab biosimilar after a relapse while on dual therapy. Clin Exp Rheumatol 2017; 35:356-357.

70 Schimmelpennink MC, Vorselaars ADM, van Beek FT, et al. Efficacy and safety of infliximab biosimilar Inflectra((R)) in severe sarcoidosis. Respir Med 2018;138S:S7-S13.

* First study demonstrating the efficacy and safety of a biosimilar in sarcoidosis.

71 Crommelin HA, van der Burg LM, Vorselaars AD, et al. Efficacy of adalimumab in sarcoidosis patients who developed intolerance to infliximab. Respir Med 2016;115:72-77.

72 Doty JD, Mazur JE, Judson MA. Treatment of corticosteroid-resistant neurosarcoidosis with a shortcourse cyclophosphamide regimen. Chest 2003;124:2023-2026.

73 Sodhi M, Pearson K, White ES, Culver DA. Infliximab therapy rescues cyclophosphamide failure in severe central nervous system sarcoidosis. Respir Med 2009;103:268-273.

74 Talar-Williams C, Hijazi YM, Walther MM, et al. Cyclophosphamide-induced cystitis and bladder cancer in patients with Wegener granulomatosis. Ann Intern Med 1996;124:477-484.

75 Lower EE, Baughman RP, Kaufman AH. Rituximab for refractory granulomatous eye disease. Clin Ophthalmol 2012;6:1613-1618.

76 Sweiss NJ, Lower EE, Mirsaeidi M, et al. Rituximab in the treatment of refractory pulmonary sarcoidosis. Eur Respir J 2014;43:1525-1528.

77 Parambil JG, Tavee JO, Zhou L, et al. Efficacy of intravenous immunoglobulin for small fiber neuropathy associated with sarcoidosis. Respir Med 2011;105:101-105.

78 Tavee JO, Karwa K, Ahmed Z, et al. Sarcoidosis-associated small fiber neuropathy in a large cohort: clinical aspects and response to IVIG and anti-TNF alpha treatment. Respir Med 2017;126:135-138.

* A very interesting article describing responses to intravenous immunogloblulin (IVIG) and anti-TNF-a treatment in sarcoidosis-associated SFN. Among 115 patients, symptomatic improvement was seen in $75 \%$ with IVIG, in $67 \%$ with anti-TNF-a, and in $71 \%$ with both treatments.

79 Culver DA, Dahan A, Bajorunas D, et al. Cibinetide improves corneal nerve fiber abundance in patients with sarcoidosis-associated small nerve fiber loss and neuropathic pain. Invest Ophthalmol Vis Sci 2017; 58:BIO52-BIO60.

80 Dahan A, Dunne A, Swartjes M, et al. ARA 290 improves symptoms in patients with sarcoidosisassociated small nerve fiber loss and increases corneal nerve fiber density. Mol Med 2013;19:334-345. 
Part II 



\section{Chapter 6}

\section{Small fiber neuropathy: a disabling and underrecognized syndrome}

M. Voortman, D. Fritz, O.J.M. Vogels, F. Eftimov, D. van de Beek, M.C. Brouwer, M. Drent Current Opinion of Pulmonology 2017:23(5):447-457 


\section{Abstract}

\section{Purpose of review}

To discuss cause, clinical manifestations, diagnostics, and treatment of small fiber neuropathy (SFN). The diagnosis is difficult and can be easily missed.

\section{Recent findings}

SFN causes high morbidity with disabling symptoms and impact on quality of life. Patients may benefit from being diagnosed with SFN, even if no underlying cause is identified and no specific treatment is yet available. Recently, genetic mutations as a possible cause of SFN were identified. Clinical diagnostic criteria have been proposed, but no gold standard exists, and each test has its limitations. The diagnosis requires a combination of typical symptoms, abnormal neurologic findings, and absence of large fiber involvement. Clinicians should be aware of overlapping symptoms of SFN and fibromyalgia. Treatment is often difficult, even when the underlying cause is identified and appropriately treated. Usually, only symptomatic relief of complaints is available.

\section{Summary}

Awareness of SFN and related symptoms is of great clinical relevance. Guidelines for appropriate diagnostic workup using a stepwise approach involving a combination of tests are warranted. Even if no treatment is available, patients may benefit from timely recognition of SFN. 


\section{Introduction}

Peripheral neuropathies can be subdivided into large-fiber neuropathy and small fiber neuropathy (SFN). When large fibers or (more commonly) a combination of large and small fibers are involved, the term polyneuropathy is used, whereas isolated involvement of small fibers is generally referred to as SFN. SFN is a generalized sensory nerve disorder with structural and functional abnormalities of small fibers, characterized histopathological by degeneration of small fiber nerve endings.

Due to lack of awareness among clinical physicians, the diagnosis of SFN is probably highly underreported. In the Netherlands, a minimum estimated incidence of 12 per 100000 population and a prevalence of 53 per 100000 population have been reported, but even this systematic assessment underestimated the incidence and prevalence. ${ }^{1}$ The diagnosis of SFN may be difficult because a diagnostic gold standard is lacking. The diagnosis is mostly made on the basis of the presence of characteristic clinical features in combination with abnormalities on neurophysiological tests and/or reduced numbers of small fibers in skin biopsy. SFN is often idiopathic or it can be an epiphenomenon in many diseases, including sarcoidosis. Treatment is often difficult, even when the underlying cause is identified. Symptomatic treatment is available, but its effectiveness is limited to a subgroup of patients with SFN. Here, we discuss the cause, clinical manifestations, diagnostics, and treatment of SFN, with special emphasis on SFN in patients with sarcoidosis.

\section{Pathophysiology}

The histopathology of SFN is characterized by denervation of the thin myelinated Adand unmyelinated C-fibers. ${ }^{2}$ As these fibers perceive thermal and nociceptive (somatic) sensations and have autonomic function, denervation results in symptoms of neuropathic pain and autonomic dysfunction. ${ }^{3}$ Morphologic changes within the axons can also be found. ${ }^{4}$ The exact pathophysiological mechanisms are often unknown and depend on the underlying illnesses associated with SFN, such as metabolic, infectious, inflammatory, and genetic diseases (Table 6.1). More recently, hereditary SFN has been described with pathogenic mutations in sodium channels (Nav1.7 (mostly), Nav1.8 and Nav1.9) which lead to hyperexcitability of dorsal root ganglions. These gain-of-function mutations result in degeneration of small fibers. ${ }^{12,13,14^{*}}$ 
Table 6.1 Causes of small fiber neuropathy.

\begin{tabular}{|c|c|}
\hline Primary & Secondary \\
\hline $\begin{array}{l}\text { idiopathic small fiber neuropathy } \\
\text { hereditary } \\
\text { mutations in sodium channels (Nav1.7, Nav1.8, } \\
\text { Nav1.9) } \\
\text { hereditary sensory neuropathy }^{8^{*}, 15} \\
\text { Fabry's disease } \\
\text { familial amyloid polyneuropathy }^{16} \\
\text { Wilson's disease }^{20,21}\end{array}$ & 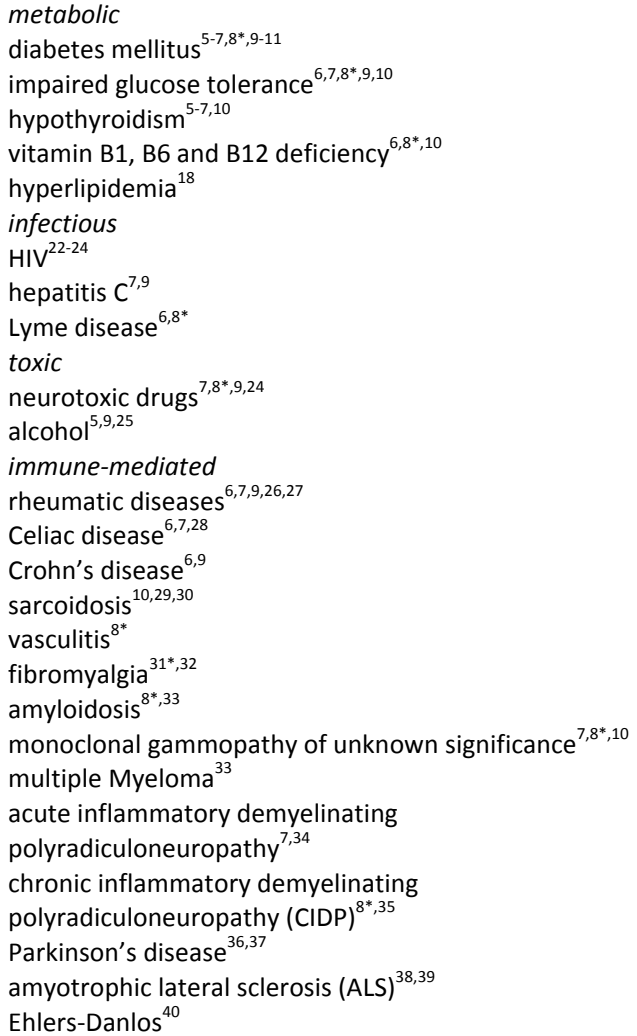 \\
\hline
\end{tabular}

The pathophysiology depends on the underlying disease. In diabetes and impaired glucose tolerance, the cause is multifactorial and includes hyperglycemia, oxidative stress, ischemia, and hypoxia. ${ }^{41,42}$ In addition, direct neurotoxic effects (HIV, chemotherapy, retroviral drugs, and alcohol) can lead to axon loss, resulting in SFN. ${ }^{22,23,42}$

In other diseases (immune-mediated diseases, sarcoidosis) inflammatory mediators, such as interleukin beta (IL1- $\beta$ ), IL6 and IL8, and tumour necrosis factor alpha (TNF- $\alpha$ ) concentrations were found to be elevated in SFN patients, suggesting an important role. $^{22,23,41,43}$ IL1- $\beta$ and TNF- $\alpha$ are also known to reduce mechanical nociceptive thresholds ${ }^{44,45}$ and have been found in higher concentrations in distal skin biopsies of patients with length-dependent SFN. ${ }^{43}$ Moreover, TNF- $\alpha$ inhibition has been reported to reduce SFN symptoms in a patient with sarcoidosis. ${ }^{46}$ TNF- $\alpha$ also plays a role in other immune-mediated neuropathies like acute inflammatory demyelinating polyradiculo- 
neuropathy, ${ }^{47}$ in which a positive correlation was found between serum levels of TNF- $\alpha$ and the severity of neuropathy.

Establishing a caused-based diagnosis is important for patients with SFN, as the underlying cause may need additional treatment and can influence prognosis. For example, patients with diabetes need to be treated with oral antidiabetics or insulin to alleviate and prevent other complications of the disease. However, despite a thorough workup, the proportion of patients with idiopathic SFN remains substantial, ranging from 23 up to $47 \%$. $^{5,7}$

\section{Symptoms}

Symptoms of SFN can vary widely in terms of distribution, severity, and progression. Usually, SFN is length-dependent, resulting in loss of function starting distally in the lower extremities. However, in some cases, it can be nonlength-dependent, resulting in a patchy distribution across the upper extremities, face, trunk, or other focal areas. ${ }^{3}$ Nonlength-dependent SFN seems to be more prevalent in autoimmune and inflammatory diseases. ${ }^{10}$ In addition, recent evidence suggests that SFN is a nonlengthdependent distal axonopathy, even in patients with a length-dependent clinical presentation. ${ }^{48}$ When small somatic fibers are affected, patients experience neuropathic pain that can be burning, deep or electric shock-like but can also have a pruritic component. ${ }^{49}$ Pain is usually continuous and stimulus-independent. However, some patients complain of evoked pain, such as allodynia, leading to intolerance of bedsheets and clothing or warm water. ${ }^{50}$ Paraesthesia, hyperesthesia, numbness, and diminished discrimination of heat and cold can also occur (Table 6.2). Patients report that symptoms worsen during rest and at night, affecting sleep, and sometimes resembling symptoms of Restless Legs Syndrome. ${ }^{2}$ The most frequent symptom of autonomic dysfunction is probably vasomotor dysregulation, presenting with fluctuating purple/blue discoloration of hands and feet. ${ }^{27}$ Other symptoms of autonomic dysfunction are gastrointestinal and urinary complaints, abnormal sweating, sicca symptoms, sexual dysfunction, arrhythmias, and (pre)syncope (see Table 6.2). It has previously been identified as a strong predictor of morbidity and mortality. ${ }^{53,54}$ Alternatively, symptoms, such as gait instability or weakness, suggest involvement of large fibers. SFN has substantial effects on patients' quality of life (QoL). ${ }^{55}$

Sarcoidosis patients with muscle and/or joint pain are often referred to a rheumatologist. If no underlying rheumatic disorder is found, the symptoms are generally associated with fibromyalgia, whereas SFN is not considered. As fibromyalgia symptoms are partially similar to those of SFN, different diagnosis (fibromyalgia or SFN) 
may be established, depending on the consulting clinician (rheumatologist or neurologist). To date, functional deficits of small fibers, as commonly found in SFN, have also been detected in patients with fibromyalgia. ${ }^{31^{*}, 56}$ Some patients with chronic pain labelled as 'fibromyalgia' may in fact have unrecognized SFN. ${ }^{52}$

Table 6.2 Symptoms of small fiber neuropathy, fibromyalgia, and peripheral large fiber polyneuropathy.

\begin{tabular}{|c|c|c|c|}
\hline & SFN & Fibromyalgia & Large fiber polyneuropathy* \\
\hline neuropathic pain & + & + & + \\
\hline paraesthesia & + & + & + \\
\hline allodynia (sheet intolerance) & + & + & + \\
\hline restless legs & + & + & + \\
\hline abnormal warm and cold sensation thresholds & + & - & + \\
\hline weakness & - & - & + \\
\hline impaired balance & - & - & + \\
\hline \multicolumn{4}{|l|}{ autonomic dysfunction } \\
\hline hypo- or hyperhidrosis & + & - & - \\
\hline defecation disturbances: diarrhea or constipation & + & + & - \\
\hline gastroparesis & + & - & - \\
\hline micturition disturbances & + & + & - \\
\hline sicca symptoms (dry eyes and/or dry mouth) & + & + & - \\
\hline blurry vision; accommodation problems & + & - & - \\
\hline hot flushes & + & - & - \\
\hline orthostatic dizziness & + & + & - \\
\hline sexual dysfunction & + & + & - \\
\hline cardiac palpitations/(pre)syncope & + & + & - \\
\hline \multicolumn{4}{|l|}{ general } \\
\hline fatigue & + & + & + \\
\hline waking up unrefreshed & - & + & - \\
\hline cognitive disturbances & - & + & - \\
\hline widespread musculoskeletal pain & - & + & - \\
\hline headache & - & + & \\
\hline temporomandibular disorder & - & + & - \\
\hline
\end{tabular}

*Polyneuropathy often involves large and small fibers leading to combination of symptoms. Adapted from. ${ }^{32,50-52}$

\section{Diagnosis of small fiber neuropathy}

The diagnosis of SFN is complicated by the lack of a gold standard. Various diseases that should be considered in the differential diagnosis, include polyneuropathy (large fiber neuropathy with or without SFN) and fibromyalgia (Table 6.2). Nerve conduction studies (NCS) are performed as the first diagnostic test to exclude large-fiber disorders. If NCS findings are normal, further diagnostic work-up is needed, although some argue that a typical clinical picture and a known cause of SFN might be sufficient. 
Several diagnostic criteria have been proposed for diagnosing SFN, ${ }^{7,57,58}$ combining clinical symptoms, abnormalities in neurological examinations, and abnormal neurophysiological test results (discussed below) or skin biopsy. These criteria enable possible, probable, or definite SFN to be diagnosed. Nevertheless, further studies are warranted to confirm the usefulness of these criteria. No final set of well-defined SFN criteria is as yet available. However, patients often benefit from a diagnosis of SFN, even if no underlying cause is identified and no specific treatment is available.

Various diagnostic tests are available for the assessment of SFN, which have mostly been validated in patients with diabetes and idiopathic SFN (Table 6.3).

\section{Small fiber neuropathy screening list}

An SFN screening tool has been developed for patients with sarcoidosis, called the SFN screening list (SFNSL), ${ }^{85}$ which was intended as a first attempt to achieve early identification of patients with symptoms related to SFN. The SFNSL consists of 21 questions (total score range 0-84). A score of less than 11 is considered indicative of a normal temperature threshold testing (TTT), while a cut-off of more than 48 is considered indicative of an abnormal TTT, indicating SFN. However, a substantial proportion of patients and controls are found to be in the intermediate group (score 11-48).

\section{Skin wrinkling test}

Skin wrinkling results from vasoconstriction controlled by the sympathetic nervous system. ${ }^{62,85,86^{*}}$ This is a simple test using warm water, a eutectic mixture of local anaesthetics or Neuropads. ${ }^{59,61}$

\section{Quantitative sensory testing}

Quantitative sensory testing is a frequently used technique involving exposure to different types of mechanical and thermal stimuli. It includes TTT, which has the highest sensitivity (Table 6.3), but mechanical detection thresholds, thermal and mechanical pain thresholds can also be tested in $\mathrm{SFN}^{64,69^{*}}$ It is an easy, non-invasive but time consuming method. 
Table 6.3 Diagnostic tests in small fiber neuropathy.

\begin{tabular}{|c|c|c|c|c|}
\hline Diagnostic test & Sensitivity & Specificity & Limitation & Sarcoidosis \\
\hline SFNSL score $<11$ & $100 \%$ & $31 \%$ & only validated in sarcoidosis & + \\
\hline SFNSL score $>48$ & $19 \%$ & $100 \%$ & & \\
\hline Skin wrinkling ${ }^{59-62}$ & $66-82 \%$ & $67-75 \%$ & $\begin{array}{l}\text { training of staff necessary, no } \\
\text { correlation with IENFD, poor } \\
\text { NPV }\end{array}$ & $\begin{array}{l}\text { no research } \\
\text { available }\end{array}$ \\
\hline QST (mainly TTT) ${ }^{7,63-67}$ & $\begin{array}{l}57-93 \% \\
\text { TTT: } 84 \%{ }^{63}\end{array}$ & $\begin{array}{l}37-94 \% \\
\text { TTT:94\% }{ }^{63}\end{array}$ & $\begin{array}{l}\text { requires conscious integration } \\
\text { of patients, difficult to } \\
\text { distinguish between faked and } \\
\text { true loss of sensation, central } \\
\text { and peripheral nervous system } \\
\text { abnormalities can lead to same } \\
\text { deficit }\end{array}$ & $\begin{array}{l}\text { sensitivity TTT } \\
69 \% \text {; } \\
\text { abnormalities } \\
\text { feet vs. hands } \\
(54 \% \text { vs. } 38 \%), \\
15 \% \text { both }^{64}\end{array}$ \\
\hline LEP/CHEP/PREP ${ }^{68-72,73^{* *}}$ & $64-94 \%$ & $69-87 \%$ & $\begin{array}{l}\text { abnormal test not only small } \\
\text { fibers (entire nociceptive } \\
\text { pathway), seasonal differences } \\
\text { in heat conductivity in CHEP } \\
\text { (less reliable outcome), limited } \\
\text { availability and needs well- } \\
\text { trained staff }\end{array}$ & $\begin{array}{l}\text { no research } \\
\text { available }\end{array}$ \\
\hline Skin biopsy/IENFD $7,29,74,75$ & $\begin{array}{l}33-90 \% \\
\text { Pure SFN: } \\
>90 \%^{2,74}\end{array}$ & $\begin{array}{l}58-95 \% \\
\text { Pure SFN: } \\
>90 \%, 74\end{array}$ & $\begin{array}{l}\text { requires training, limited } \\
\text { availability/not generally } \\
\text { distributed (only a few centers), } \\
\text { unclear in case of patchy } \\
\text { disease, time consuming }\end{array}$ & $\begin{array}{l}\text { sensitivity of } \\
32.8 \% \text { with age- } \\
\text { dependent } \\
\text { decrease (men } \\
<\text { women) }\end{array}$ \\
\hline $\mathrm{CCM} \mathrm{CNFD}^{76^{*}, 77^{*}, 78,79}$ & $50-86 \%$ & $52-84 \%$ & $\begin{array}{l}\text { not widely available, high } \\
\text { acquisition costs, uncertainties } \\
\text { about age-related decline and } \\
\text { racial differences in corneal } \\
\text { nerve measurements }\end{array}$ & $\begin{array}{l}50 \% \text { reduction in } \\
\text { corneal nerve } \\
\text { fibers in painful } \\
\text { SFN compared to } \\
\text { healthy } \\
\text { controls }^{77^{*}}\end{array}$ \\
\hline \multicolumn{5}{|l|}{ Autonomic function } \\
\hline CAFT & low & & & $\begin{array}{l}\text { abnormal in only } \\
12.5 \% \text { of SSFN } \\
\text { patients }^{80}\end{array}$ \\
\hline QSART $^{66,81,82}$ & $59-80 \%$ & - & & no research \\
\hline Sudoscan $^{83,84}$ & $65-78 \%$ & $80-92 \%$ & & $\begin{array}{l}\text { available on } \\
\text { QSART or } \\
\text { sudoscan. SSR } \\
\text { abnormal in } \\
<10 \% \text { of SSFN }\end{array}$ \\
\hline MIBG-scan & & & $\begin{array}{l}\text { no standardized methodology } \\
\text { or normative values, } \\
\text { discontinuation of some drugs } \\
\text { is needed }\end{array}$ & $\begin{array}{l}>50 \% \\
\text { involvement } \\
\text { cardiac } \\
\text { autonomic }_{\text {dysfunction }}^{80}\end{array}$ \\
\hline
\end{tabular}

$\mathrm{CAFT}=$ cardiovascular autonomic testing; $\mathrm{CCM}=$ corneal confocal microscopy; $\mathrm{CHEP}=$ contact heat-evoked potential; $C N F D=$ corneal nerve-fiber density; IENFD=intraepidermal nerve fiber density; LEP=laser-evoked potential; MIBG-scan=metaiodobenzylguanidine-scan; NPV=negative predictive value; PREP=pain-related evoked potential; QSART=quantitative sudomotor axon reflex test; QST=quantitative sensory testing; SFNSL=small fiber neuropathy screening list; SSFN=sarcoidosis small fiber neuropathy; TTT=temperature threshold test. 


\section{Nociceptive-evoked potentials}

Nociceptive-evoked potentials include laser-evoked potential (LEP), contact heatevoked potential (CHEP), and pain-related evoked potential (PREP). Both LEP and CHEP result from selective activation of Ad-fibers and C-fibers, whereas PREP results from activation of only Ad-fibers. ${ }^{87}$ The amplitude potential correlates inversely with the reported pain intensity. ${ }^{68,70}$

\section{Skin biopsy}

A 3-mm punch biopsy is obtained from the distal part of the leg $(10 \mathrm{~cm}$ above the malleolus). This is a minimally invasive technique. Decreased intraepidermal nerve-fiber density (IENFD) does not correlate with neuropathic pain. ${ }^{88}$ In addition to decreased IENFD, axonal swelling - an early marker of SFN - or decreased sweat gland innervation can be found in SFN. ${ }^{4,75,89}$

\section{Corneal confocal microscopy}

The cornea is innervated by Ad-fibers and C-fibers, originating from the ophthalmic branch of the trigeminal nerve. Corneal confocal microscopy is a reproducible, noninvasive clinical technique detecting early nerve damage. ${ }^{79,90^{*}, 91}$ Correlation has been established between severity of neuropathy and progressive corneal nerve degeneration. $^{79,92}$

\section{Autonomic function tests}

Autonomic dysregulation can contribute to fatal arrhythmias and unexplained sudden death and involvement of small autonomic fibers predicts cardiovascular mortality. ${ }^{53,93}$ Different diagnostic modalities can be used, mainly to establish the severity of the complaints. SFN involvement may be unrecognized in sarcoidosis patients with cardiac symptoms.

1. Cardiovascular autonomic function test (CAFT): cardiovascular autonomic function can be assessed using five tests (Ewing tests), establishing heart rate variability and blood pressure in supine position, in upright position, during deep respiration, during Valsalva maneuver, and while rapidly changing position from supine to upright. CAFT is considered abnormal when at least two of these five tests are abnormal. ${ }^{64}$ It is easy to perform but has low sensitivity in SFN. ${ }^{64,82}$

2. Sudomotor and vasodilator function tests: the most commonly used sudomotor function test is quantitative sudomotor axon reflex test (QSART). QSART evaluates the volume and rate of sweat production (reflecting small C-fiber function). As it is 
a time-consuming technique, a new device called sudoscan was developed, which is easy to perform and rapid. This technique has been used in various studies to detect SFN in patients with diabetes and Fabry disease. ${ }^{69^{*}, 84,94^{*}, 95}$

3. Sympathetic skin response (SSR), thermoregulatory sweat test (TST), skin vasomotor reflex (SVR): these tests are not frequently used, due to difficulties of assessment (TST), poor reproducibility (SSR), and low sensitivity and specificity (SSR and SVR). ${ }^{64,69 *, 96,97}$

4. Metaiodobenzylguanidine (MIBG)-scintigraphy: this imaging technique may be a promising new tool to study the heart's innervation, currently mainly used in clinical studies. Cardiac sympathetic dysfunction and related symptoms have been reported in patients with SFN. When cardiac symptoms are present in SFN patients, the myocardial sympathetic nervous system, and thus autonomic function ${ }^{80}$ can be assessed by iodine-123 MIBG (I-123 MIBG) scintigraphy (Figure 6.1). I-123 MIBG is a norepinephrine analog which has identical mechanisms of storage, uptake, and release in neurons. MIBG scintigraphy can show reduced I-123 MIBG uptake in the myocardium, regional defects, or a higher washout of I-123 MIBG. ${ }^{80,98,99 *, 100-102}$ Cardiac sympathetic dysfunction seems to be heterogeneous and dependent on the presence or absence of SFN. ${ }^{80}$ I-123 MIBG can unravel, so far unknown, relationships between innervation, vascularization, and endothelial integrity. Other diagnostic tools, such as MRI and CT, do not have this capacity. In some symptomatic cases, both FDG PET and MRI can be normal, whereas I-123 MIBG scintigraphy is abnormal. ${ }^{103}$

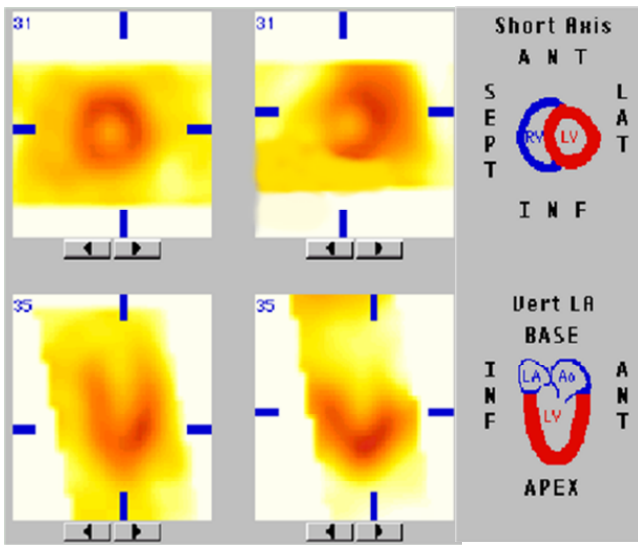

Thallium

MIBG

Figure 6.1 Thallium versus MIBG scintigraphy of a sarcoidosis patient with collapses and palpitations demonstrating impaired I-123 metaiodobenzylguanidine uptake without myocardial perfusion disturbances found by thallium scintigraphy. Part of this figure was previously published in ref ${ }^{80}$. 


\section{Treatment}

Treatment of SFN depends on the underlying disease, if identified. Symptoms are often disabling and difficult to treat, even when the cause is identified and adequately treated, leading to high morbidity and decreased QoL. ${ }^{55}$ Usually, only symptomatic relief of complaints is achieved. Consensus guidelines for neuropathic pain have been adapted for the treatment of pain in SFN.

\section{Neuropathic pain}

Symptomatic treatment prescribed for neuropathic pain includes antidepressants (TCAs and selective serotonin norepinephrine reuptake inhibitors [SSNRIs]), anticonvulsants, opioids, and topical drugs (Table 6.4). Unfortunately, these drugs often provide only partial relief from pain, have no effect on autonomic dysfunction and are associated with (sometimes severe) side effects in a high proportion of patients. Treatment of neuropathic pain is mainly based on studies of large-fiber neuropathies, only one study having examined SFN. ${ }^{105}$ This randomized controlled study with crossover design involving 18 patients showed that gabapentin and tramadol significantly reduced pain scores ( $p=0.001$ and 0.018 , respectively).

Amitriptyline is a TCA and is the first-line treatment for neuropathic pain, mainly based on years of experience. However, unbiased supportive studies evaluating the effect of amitriptyline are lacking, and the effect might be overestimated. ${ }^{106}$ Gabapentin and pregabalin have been shown to be effective in painful neuropathies in randomized controlled studies. ${ }^{50,107-109}$ Overall, these drugs are well tolerated. Other anticonvulsant drugs have been found to have little to no effect, apart from certain indications (topiramate and lamotrigine) ${ }^{110,111}$ or have low-quality evidence with high risk of bias (carbamazepine). ${ }^{112}$ Of the SSNRIs, duloxetine has been found to be a more potent inhibitor than venlafaxine, and is therefore preferred. ${ }^{42}$ Selective serotonin reuptake inhibitors are not recommended, due to limited data on their efficacy. Tramadol and oxycodone can reduce pain in diabetic painful neuropathies and can be used for breakthrough pain. ${ }^{107}$ However, they should be avoided due to risk of addiction and tolerance to their analgesic effect.

Topical treatment can be an option in localized neuropathic pain. Two drugs have been registered for this indication, namely lidocaine $5 \%$ patches ${ }^{113^{*}}$ and capsaicin $8 \%$ patches. Capsaicin has proven to be effective in diabetic and HIV neuropathy, reducing pain by $22.8-40 \%{ }^{113^{*}}$ A retrospective study also found $75 \%$ reduction of pain intensity. ${ }^{114}$ It can be a good alternative to oral drugs, with lesser side effects. 
Table 6.4 Medical treatment for small fiber neuropathy.

\begin{tabular}{|c|c|c|}
\hline Drug & $\begin{array}{c}\text { Dosage } \\
\text { recommendations }\end{array}$ & Common side effect \\
\hline \multicolumn{3}{|l|}{ antidepressants } \\
\hline amitriptyline (TCA, first line) & $20-150 \mathrm{mg}$ & $\begin{array}{l}\text { anticholinergic effects, sedation, nausea, } \\
\text { weight gain, palpitations }\end{array}$ \\
\hline nortriptyline (TCA, first line) & $20-150 \mathrm{mg}$ & $\begin{array}{l}\text { anticholinergic effects, sedation, nausea, } \\
\text { weight gain }\end{array}$ \\
\hline desipramine (TCA, first line) & $20-200 \mathrm{mg}$ & $\begin{array}{l}\text { anticholinergic effects, sedation, nausea, } \\
\text { weight gain }\end{array}$ \\
\hline duloxetine (SSNRI, first line) & $30-120 \mathrm{mg}$ & $\begin{array}{l}\text { nausea, sedation, dizziness, headache, weight } \\
\text { loss }\end{array}$ \\
\hline venlafaxine (SSNRI, first line) & $150-225 \mathrm{mg}$ & dizziness, headache, nausea, perspiration \\
\hline \multicolumn{3}{|l|}{ anticonvulsants } \\
\hline gabapentin (first line) & $300-3600 \mathrm{mg}$ & somnolence, dizziness, ataxia, edema, tremor \\
\hline pregabalin (first line) & $150-600 \mathrm{mg}$ & $\begin{array}{l}\text { somnolence (less), dizziness, ataxia, edema, } \\
\text { tremor }\end{array}$ \\
\hline topiramate (third line) & $25-400 \mathrm{mg}$ & $\begin{array}{l}\text { weight loss, sedation, diarrhea, nausea, } \\
\text { dizziness, paraesthesia }\end{array}$ \\
\hline lamotrigine (third line) & $25-400 \mathrm{mg}$ & $\begin{array}{l}\text { headache, rash, dizziness, nausea, sedation, } \\
\text { tremor }\end{array}$ \\
\hline carbamazepine (third line) & $200-1200 \mathrm{mg}$ & $\begin{array}{l}\text { dizziness, ataxia, nausea, vomiting, sedation, } \\
\text { rash, liver-enzymes elevation }\end{array}$ \\
\hline \multicolumn{3}{|l|}{ opioids } \\
\hline tramadol (second line) & $50-400 \mathrm{mg}$ & $\begin{array}{l}\text { constipation, pruritus, dizziness, nausea, } \\
\text { vomiting, sedation, impaired concentration, } \\
\text { ataxia }\end{array}$ \\
\hline oxycodon (third line) & $10-100 \mathrm{mg}$ & $\begin{array}{l}\text { constipation, pruritus, dizziness, nausea, } \\
\text { vomiting, sedation, impaired concentration, } \\
\text { ataxia, potential addiction abuse }\end{array}$ \\
\hline \multicolumn{3}{|l|}{ topical } \\
\hline capsaicin $8 \%$ patch (second line) & 3-4 times daily & burning sensation, erythema, local edema \\
\hline lidocaine $5 \%$ plaster (second line) & 2 times daily & burning sensation, erythema, local edema, rash \\
\hline \multicolumn{3}{|l|}{ other } \\
\hline immunoglobulins (clinical trial stage) & $\begin{array}{l}\text { Loading dose } 2 \mathrm{~g} / \mathrm{kg} \text {, } \\
\text { maintenance } 0.75-1 \\
\mathrm{~g} / \mathrm{kg} \text { every } 3 \text { weeks }\end{array}$ & $\begin{array}{l}\text { hypertension, headache, nausea, vomiting, } \\
\text { diarrhea, rash, flu-like disease }\end{array}$ \\
\hline infliximab (TNF- $\alpha$ inhibition) & $\begin{array}{l}3 \mathrm{mg} / \mathrm{kg} \text { every } 4-6 \\
\text { weeks }\end{array}$ & $\begin{array}{l}\text { infections, fever, headache, dizziness, } \\
\text { paraesthesias, tachycardia, bone marrow } \\
\text { depression, liver-enzymes elevation }\end{array}$ \\
\hline cibinetide (ARA290, preclinical stage) & $\begin{array}{c}2 \mathrm{mg} 3 \text { times/week } \\
4 \mathrm{mg} \text { daily }\end{array}$ & diarrhea \\
\hline
\end{tabular}

TCA=tricyclic antidepressant; SSNRI=serotonin norepinephrine reuptake inhibitor; TNF- $\alpha=$ tumour necrosis factor alpha. Adapted from ${ }^{104 * *}$

Different diseases causing SFN may respond differently to these drugs. In HIV neuropathy, amitriptyline, topical lidocaine, and pregabalin failed to relieve pain. ${ }^{115-118}$ 
Only lamotrigine and capsaicin were found to be moderately effective. ${ }^{107}$ In chemotherapy-induced neuropathy, no evidence was found for the efficacy of amitriptyline, nortriptyline, or gabapentin. ${ }^{119-121}$

Immunoglobulins and TNF- $\alpha$ inhibition have been reported to be effective in patients with SFN with various causes. ${ }^{46,122-128}$ Recently, a large retrospective cohort study of sarcoidosis associated SFN found that immunoglobulins and TNF- $\alpha$ inhibition caused improvement in 75 and $67 \%$ of cases, respectively. ${ }^{129^{*}}$ Whether these very expensive treatments should be initiated as causative treatment for SFN is unclear and currently being investigated. Cibinetide (ARA290) seems a promising new drug to relieve pain and increase corneal and skin-nerve-fiber density ${ }^{77^{*}, 130,131}$ in sarcoid and diabetic neuropathy. Further clinical trials are needed before this drug can be registered.

\section{Autonomic dysfunction}

Treatment of autonomic neuropathy depends on the type of symptoms patients report. In patients with cardiac autonomic neuropathy (arrhythmias), improvement can be obtained with carvedilol. ${ }^{132,133}$ Treatment of postural orthostatic hypotension should involve stopping or modifying medication that can cause or worsen orthostatic hypotension and start nonpharmacological measures (increased salt intake, stockings, etc.). Pharmacologic treatment can consist of midodrine ${ }^{134,135}$ or droxidopa. ${ }^{136,137}$ Hyperhidrosis may respond to clonidine, amitriptyline, or botulin toxin. ${ }^{138}$

\section{Discussion}

SFN is an underrecognized clinical condition that has been associated with substantial burden on individuals' QoL. Patients often feel misunderstood and are limited in daily activities by their complaints. SFN can be caused by multiple underlying diseases, of which diabetes mellitus is the most frequent (Table 6.1). Studies of the true incidence and systematic reports on underlying causes of SFN have been scarce. ${ }^{5-7}$ Extensive screening for underlying disease and the detection of heritability as a possible cause of SFN has reduced the proportion of patients with idiopathic SFN to approximately half of diagnosed patients.

A protocolled approach to diagnosing SFN is of great importance to rule out treatable causes of SFN and differentiate it from large-fiber neuropathy and fibromyalgia (Table 6.2). The diagnosis of SFN can be difficult and no gold standard is available. Unlike in large-fiber neuropathy, NCS are generally normal in SFN. 
All diagnostic modalities summarized in this review have their limitations, although skin biopsy is considered by some to be the 'silver standard'. What is important, however, is that a normal skin biopsy does not completely exclude SFN, possibly due to its sometimes patchy distribution. Moreover, skin biopsy does not always correlated with complaints. $^{75,88}$ Just as in diagnosing idiopathic pulmonary fibrosis, we consider combining multiple diagnostic tests in a consecutive order to be the best option, although the best combination and sequence of tests remain to be determined. Diagnostic criteria have been suggested, and skin biopsy will not always be necessary. ${ }^{57,58,64,72,76^{*}, 139}$

Treatment of SFN depends on the underlying illness, and is generally difficult. Responses to treatment are poor-to-moderate, and many patients experience side effects. The question remains for which patient treatment should be initiated, and at what stage. Most importantly, a multidisciplinary approach is recommended and more collaboration between centers of excellence is necessary.

\section{Conclusion}

SFN is a disabling generalized sensory nerve disorder with a widespread spectrum of symptoms, affecting QoL. The disorder can accompany many different diseases. SFN is difficult to diagnose, as a gold standard is still lacking. There is a need for additional reliable, valid, and responsive tests to diagnose SFN, as well as a clinical diagnostic guideline using a stepwise approach and a combination of tests. Treating the underlying disease is only effective in some cases. In the majority of cases, the only option is symptomatic treatment, which usually produces only partial pain relief. Identifying and understanding the pathological basis of SFN will facilitate further studies on diagnostic methods and novel treatment approaches. Prospective research is warranted to improve the diagnostic process, treatment, and outcome. 


\section{References}

* of special interest

** of outstanding interest

1 Peters MJ, Bakkers M, Merkies IS, et al. Incidence and prevalence of small-fiber neuropathy: a survey in the Netherlands. Neurology 2013;81:1356-1360.

2 Lauria G, Lombardi R. Small fiber neuropathy: is skin biopsy the holy grail? Curr Diab Rep 2012;12: 384-392.

3 Themistocleous AC, Ramirez JD, Serra J, Bennett DL. The clinical approach to small fibre neuropathy and painful channelopathy. Pract Neurol 2014;14:368-379.

4 Lauria G, Morbin M, Lombardi R, et al. Axonal swellings predict the degeneration of epidermal nerve fibers in painful neuropathies. Neurology 2003;61:631-636.

5 Bednarik J, Vlckova-Moravcova E, Bursova S, et al. Etiology of small-fiber neuropathy. J Peripher Nerv Syst 2009;14:177-183.

6 De Sousa EA, Hays AP, Chin RL, et al. Characteristics of patients with sensory neuropathy diagnosed with abnormal small nerve fibres on skin biopsy. J Neurol Neurosurg Psychiatry 2006;77:983-985.

7 Devigili G, Tugnoli V, Penza P, et al. The diagnostic criteria for small fibre neuropathy: from symptoms to neuropathology. Brain 2008;131:1912-1925.

8 Farhad K, Traub R, Ruzhansky KM, Brannagan TH, 3rd. Causes of neuropathy in patients referred as "idiopathic neuropathy". Muscle Nerve 2016; 53:856-861.

* In this study the authors reviewed the charts of 373 patients with idiopathic neuropathy and found causes in $67.3 \%$ of them, the most common being impaired glucose metabolism. Currently only abstract available.

9 Gemignani F, Giovanelli M, Vitetta F, et al. Non-length dependent small fiber neuropathy. A prospective case series. J Peripher Nerv Syst 2010;15:57-62.

10 Khan S, Zhou L. Characterization of non-length-dependent small-fiber sensory neuropathy. Muscle Nerve 2012;45:86-91.

11 Loseth S, Stalberg EV, Lindal S, et al. Small and large fiber neuropathy in those with type 1 and type 2 diabetes: a 5-year follow-up study. J Peripher Nerv Syst 2016;21:15-21.

12 Faber CG, Hoeijmakers JG, Ahn HS, et al. Gain of function Nav1.7 mutations in idiopathic small fiber neuropathy. Ann Neurol 2012;71:26-39.

13 Faber CG, Lauria G, Merkies IS, et al. Gain-of-function Nav1.8 mutations in painful neuropathy. Proc Natl Acad Sci U S A 2012;109:19444-19449.

14 Hoeijmakers JG, Faber CG, Merkies IS, Waxman SG. Painful peripheral neuropathy and sodium channel mutations. Neurosci Lett 2015;596:51-59.

* A mini-review of recently discovered sodium channel mutations in painful peripheral neuropathy.

15 Samuelsson K, Kostulas K, Vrethem M, et al. Idiopathic small fiber neuropathy: phenotype, etiologies, and the search for fabry disease. J Clin Neurol 2014;10:108-118.

16 Biegstraaten M, Hollak CE, Bakkers M, et al. Small fiber neuropathy in Fabry disease. Mol Genet Metab 2012;106:135-141.

17 Uceyler N, Kahn AK, Kramer D, et al. Impaired small fiber conduction in patients with Fabry disease: a neurophysiological case-control study. BMC Neurol 2013;13:47.

18 McManis PG, Windebank AJ, Kiziltan M. Neuropathy associated with hyperlipidemia. Neurology 1994; 44:2185-2186.

19 Ng Wing Tin S, Plante-Bordeneuve V, Salhi $\mathrm{H}$, et al. Characterization of pain in familial amyloid polyneuropathy. J Pain 2015;16:1106-1114.

20 Gondim Fde A, Araujo DF, Oliveira IS, Vale OC. Small fiber dysfunction in patients with Wilson's disease. Arq Neuropsiquiatr 2014;72:592-595. 
21 Sturniolo GC, Lazzarini D, Bartolo O, et al. Small fiber peripheral neuropathy in Wilson disease: an in vivo documentation by corneal confocal microscopy. Invest Ophthalmol Vis Sci 2015;56:1390-1395.

22 Kamerman PR, Moss PJ, Weber J, et al. Pathogenesis of HIV-associated sensory neuropathy: evidence from in vivo and in vitro experimental models. J Peripher Nerv Syst 2012;17:19-31.

23 Mangus LM, Dorsey JL, Laast VA, et al. Unraveling the pathogenesis of HIV peripheral neuropathy: insights from a simian immunodeficiency virus macaque model. ILAR J 2014;54:296-303.

24 Polydefkis M, Yiannoutsos CT, Cohen BA, et al. Reduced intraepidermal nerve fiber density in HIVassociated sensory neuropathy. Neurology 2002;58:115-119.

25 Mellion ML, Silbermann E, Gilchrist JM, et al. Small-fiber degeneration in alcohol-related peripheral neuropathy. Alcohol Clin Exp Res 2014;38:1965-1972.

26 Nguyen TP, Bangert C, Biliciler S, et al. Dermatomyositis-associated sensory neuropathy: a unifying pathogenic hypothesis. J Clin Neuromuscul Dis 2014;16:7-11.

27 Sene D, Cacoub P, Authier FJ, et al. Sjogren syndrome-associated small fiber neuropathy: characterization from a prospective series of 40 cases. Medicine (Baltimore) 2013.

28 Chin RL, Latov N. Peripheral neuropathy and celiac disease. Curr Treat Options Neurol 2005;7:43-48.

29 Bakkers M, Merkies IS, Lauria G, et al. Intraepidermal nerve fiber density and its application in sarcoidosis. Neurology 2009;73:1142-1148.

30 Hoitsma E, Marziniak M, Faber CG, et al. Small fibre neuropathy in sarcoidosis. Lancet 2002;359: 2085-2086.

31 Caro XJ, Winter EF. Evidence of abnormal epidermal nerve fiber density in fibromyalgia: clinical and immunologic implications. Arthritis Rheumatol 2014;66:1945-1954.

* An interesting study of FM patients compared to healthy controls, finding that IENFD was significantly diminished and was inversely correlated with serum levels of IL-2R.

32 Kosmidis ML, Koutsogeorgopoulou L, Alexopoulos $\mathrm{H}$, et al. Reduction of intraepidermal nerve fiber density (IENFD) in the skin biopsies of patients with fibromyalgia: a controlled study. J Neurol Sci 2014; 347:143-147.

33 Sturm D, Schmidt-Wilcke T, Greiner T, et al. Confocal cornea microscopy detects involvement of corneal nerve fibers in a patient with light-chain amyloid neuropathy caused by multiple myeloma: a case report. Case Rep Neurol 2016;8:134-139.

34 Martinez V, Fletcher D, Martin F, et al. Small fibre impairment predicts neuropathic pain in GuillainBarre syndrome. Pain 2010;151:53-60.

35 Schneider C, Bucher F, Cursiefen C, et al. Corneal confocal microscopy detects small fiber damage in chronic inflammatory demyelinating polyneuropathy (CIDP). J Peripher Nerv Syst 2014;19:322-327.

36 Kass-lliyya L, Javed S, Gosal D, et al. Small fiber neuropathy in Parkinson's disease: a clinical, pathological and corneal confocal microscopy study. Parkinsonism Relat Disord 2015;21:1454-1460.

37 Podgorny PJ, Suchowersky O, Romanchuk KG, Feasby TE. Evidence for small fiber neuropathy in early Parkinson's disease. Parkinsonism Relat Disord 2016;28:94-99.

38 Dalla Bella E, Lombardi R, Porretta-Serapiglia C, et al. Amyotrophic lateral sclerosis causes small fiber pathology. Eur J Neurol 2016;23:416-420.

39 Weis J, Katona I, Muller-Newen G, et al. Small-fiber neuropathy in patients with ALS. Neurology 2011; 76:2024-2029.

40 Cazzato D, Castori M, Lombardi R, et al. Small fiber neuropathy is a common feature of Ehlers-Danlos syndromes. Neurology 2016;87:155-159.

41 Edwards JL, Vincent AM, Cheng HT, Feldman EL. Diabetic neuropathy: mechanisms to management. Pharmacol Ther 2008;120:1-34.

42 Zeng L, Alongkronrusmee D, van Rijn RM. An integrated perspective on diabetic, alcoholic, and druginduced neuropathy, etiology, and treatment in the US. J Pain Res 2017;10:219-228.

43 Uceyler N, Kafke W, Riediger N, et al. Elevated proinflammatory cytokine expression in affected skin in small fiber neuropathy. Neurology 2010;74:1806-1813.

44 Cunha FQ, Poole S, Lorenzetti BB, Ferreira SH. The pivotal role of tumour necrosis factor alpha in the development of inflammatory hyperalgesia. Br J Pharmacol 1992;107:660-664.

45 Ferreira SH, Lorenzetti BB, Bristow AF, Poole S. Interleukin-1 beta as a potent hyperalgesic agent antagonized by a tripeptide analogue. Nature 1988;334:698-700. 
Hoitsma E, Faber CG, van Santen-Hoeufft M, et al. Improvement of small fiber neuropathy in a sarcoidosis patient after treatment with infliximab. Sarcoidosis Vasc Diffuse Lung Dis 2006;23:73-77.

47 Putzu GA, Figarella-Branger D, Bouvier-Labit C, et al. Immunohistochemical localization of cytokines, C5b-9 and ICAM-1 in peripheral nerve of Guillain-Barre syndrome. J Neurol Sci 2000;174:16-21.

48 Khoshnoodi MA, Truelove S, Burakgazi A, et al. Longitudinal assessment of small fiber neuropathy: evidence of a non-length-dependent distal axonopathy. JAMA Neurol 2016;73:684-690.

49 Brenaut E, Marcorelles P, Genestet S, et al. Pruritus: an underrecognized symptom of small-fiber neuropathies. J Am Acad Dermatol 2015;72:328-332.

50 Hovaguimian A, Gibbons $\mathrm{CH}$. Diagnosis and treatment of pain in small-fiber neuropathy. Curr Pain Headache Rep 2011;15:193-200.

51 Civelek GM, Ciftkaya PO, Karatas M. Evaluation of restless legs syndrome in fibromyalgia syndrome: an analysis of quality of sleep and life. J Back Musculoskelet Rehabil 2014;27:537-544.

52 Oaklander AL, Herzog ZD, Downs HM, Klein MM. Objective evidence that small-fiber polyneuropathy underlies some illnesses currently labeled as fibromyalgia. Pain 2013;154:2310-2316.

53 Ewing DJ, Boland O, Neilson JM, et al. Autonomic neuropathy, QT interval lengthening, and unexpected deaths in male diabetic patients. Diabetologia 1991;34:182-185.

54 Vinik Al, Ziegler D. Diabetic cardiovascular autonomic neuropathy. Circulation 2007;115:387-397.

55 Bakkers M, Faber CG, Hoeijmakers JG, et al. Small fibers, large impact: quality of life in small-fiber neuropathy. Muscle Nerve 2014;49:329-336.

56 Uceyler N, Zeller D, Kahn AK, et al. Small fibre pathology in patients with fibromyalgia syndrome. Brain 2013;136:1857-1867.

57 Blackmore D, Siddiqi ZA. Diagnostic criteria for small fiber neuropathy. J Clin Neuromuscul Dis 2017; 18:125-131.

58 Tesfaye S, Boulton AJ, Dyck PJ, et al. Diabetic neuropathies: update on definitions, diagnostic criteria, estimation of severity, and treatments. Diabetes Care 2010;33:2285-2293.

59 Datema M, Gert van Dijk J, Hoitsma E. The diagnostic value of water immersion skin wrinkling and Neuropads in small fiber neuropathy. Clin Neurophysiol 2012;123:2074-2079.

60 Ping Ng KW, Ong JJ, Nyein Nyein TD, et al. EMLA-induced skin wrinkling for the detection of diabetic neuropathy. Front Neurol 2013;4:126.

61 Teoh HL, Chow A, Wilder-Smith EP. Skin wrinkling for diagnosing small fibre neuropathy: comparison with epidermal nerve density and sympathetic skin response. J Neurol Neurosurg Psychiatry 2008; 79:835-837.

62 Wilder-Smith EP, Guo Y, Chow A. Stimulated skin wrinkling for predicting intraepidermal nerve fibre density. Clin Neurophysiol 2009;120:953-958.

63 Bakkers M, Faber CG, Reulen JP, et al. Optimizing temperature threshold testing in small-fiber neuropathy. Muscle Nerve 2015;51:870-876.

64 Hoitsma E, Drent M, Verstraete $E$, et al. Abnormal warm and cold sensation thresholds suggestive of small-fibre neuropathy in sarcoidosis. Clin Neurophysiol 2003 114:2326-2333.

65 Magda P, Latov N, Renard MV, Sander HW. Quantitative sensory testing: high sensitivity in small fiber neuropathy with normal NCS/EMG. J Peripher Nerv Syst 2002;7:225-228.

66 Periquet MI, Novak V, Collins MP, et al. Painful sensory neuropathy: prospective evaluation using skin biopsy. Neurology 1999;53:1641-1647.

67 Scott K, Simmons Z, Kothari MJ. A comparison of quantitative sensory testing with skin biopsy in small fiber neuropathy. J Clin Neuromuscul Dis 2003;4:129-132.

68 Katsarava Z, Yaldizli O, Voulkoudis C, et al. Pain related potentials by electrical stimulation of skin for detection of small-fiber neuropathy in HIV. J Neurol 2006;253:1581-1584.

69 Lefaucheur JP, Wahab A, Plante-Bordeneuve V, et al. Diagnosis of small fiber neuropathy: a comparative study of five neurophysiological tests. Neurophysiol Clin 2015;45:445-455.

* Comparison of diagnostic value of 5 neurophysiological tests (warm and cold detection threshold (WDT, CDT), LEP, SSR and sudoscan) showed that LEP is most sensitive (79\%), followed by sudoscan (61\%), WDT (55\%), SSR (41\%) and CDT (32\%).

70 Mueller D, Obermann M, Koeppen S, et al. Electrically evoked nociceptive potentials for early detection of diabetic small-fiber neuropathy. Eur J Neurol 2010;17:834-841. 
71 Parson HK, Nguyen VT, Orciga MA, et al. Contact heat-evoked potential stimulation for the evaluation of small nerve fiber function. Diabetes Technol Ther 2013;15:150-157.

72 Rage M, Van Acker N, Knaapen MW, et al. Asymptomatic small fiber neuropathy in diabetes mellitus: investigations with intraepidermal nerve fiber density, quantitative sensory testing and laser-evoked potentials. J Neurol 2011;258:1852-1864.

73 Wu SW, Wang YC, Hsieh PC, et al. Biomarkers of neuropathic pain in skin nerve degeneration neuropathy: contact heat-evoked potentials as a physiological signature. Pain 2017;158:516-525.

** This recent research paper recruited 188 patients with length-dependent sensory symptoms and reduced IENFD, to perform CHEP, QST, and NCS. CHEP amplitudes showed the highest sensitivity for diagnosing small-fiber sensory nerve degeneration. CHEP performed better in terms of diagnosing SFN than thermal thresholds and is strongly correlated with the degree of skin innervation.

74 Koskinen $M$, Hietaharju $A$, Kylaniemi $M$, et al. A quantitative method for the assessment of intraepidermal nerve fibers in small-fiber neuropathy. J Neurol 2005;252:789-794.

75 Lauria G, Hsieh ST, Johansson O, et al. European Federation of Neurological Societies/Peripheral Nerve Society Guideline on the use of skin biopsy in the diagnosis of small fiber neuropathy. Report of a joint task force of the European Federation of Neurological Societies and the Peripheral Nerve Society. Eur J Neurol 2010;17:903-912, e944-909.

76 Chen X, Graham J, Dabbah MA, et al. Small nerve fiber quantification in the diagnosis of diabetic sensorimotor polyneuropathy: comparing corneal confocal microscopy with intraepidermal nerve fiber density. Diabetes Care 2015;38:1138-1144.

* This study was performed to determine the diagnostic performance of CCM and IENFD in patients with type 1 diabetes. It showed that their diagnostic efficiency is comparable (area under the receiver operating characteristic curve was 0.80 for automated CCM and 0.66 for IENFD).

77 Dahan A, Dunne A, Swartjes M, et al. ARA 290 improves symptoms in patients with sarcoidosisassociated small nerve fiber loss and increases corneal nerve fiber density. Mol Med 2013;19:334-345.

* A single-site, double-blind, placebo-controlled trial of ARA-290 in patients with sarcoidosis-associated small nerve fiber loss. The study included 41 patients, randomized to ARA-290 or placebo. ARA-290 administration significantly improved neuropathic symptoms and increased CNFD and exercise capacity (6MWT).

78 Quattrini C, Tavakoli M, Kallinikos P, et al. Comparing skin biopsy with corneal confocal microscopy: diagnostic yield of nerve fiber density. Diabetologia 2010;53:A1114.

79 Tavakoli M, Quattrini C, Abbott C, et al. Corneal confocal microscopy: a novel noninvasive test to diagnose and stratify the severity of human diabetic neuropathy. Diabetes Care 2010;33:1792-1797.

80 Hoitsma E, Faber CG, van Kroonenburgh MJ, et al. Association of small fiber neuropathy with cardiac sympathetic dysfunction in sarcoidosis. Sarcoidosis Vasc Diffuse Lung Dis 2005;22:43-50.

81 Low PA, Tomalia VA, Park KJ. Autonomic function tests: some clinical applications. J Clin Neurol 2013; 9:1-8.

82 Novak V, Freimer ML, Kissel JT, et al. Autonomic impairment in painful neuropathy. Neurology 2001; 56:861-868.

83 Casellini CM, Parson HK, Richardson MS, et al. Sudoscan, a noninvasive tool for detecting diabetic small fiber neuropathy and autonomic dysfunction. Diabetes Technol Ther 2013;15:948-953.

84 Selvarajah D, Cash T, Davies J, et al. SUDOSCAN: a simple, rapid, and objective method with potential for screening for diabetic peripheral neuropathy. PLoS One 2015;10:e0138224.

85 Hoitsma E, De Vries J, Drent M. The small fiber neuropathy screening list: construction and crossvalidation in sarcoidosis. Respir Med 2011;105:95-100.

86 Wilder-Smith EP. Stimulated skin wrinkling as an indicator of limb sympathetic function. Clin Neurophysiol 2015;126:10-16.

* A systematic review of stimulated skin wrinkling (SSW) concluding that these is reliable support for the use of SSW, but standardized test conditions and determination of age matched cut-off values for normal and abnormal are necessary for the test to be useful.

87 Mainka T, Maier C, Enax-Krumova EK. Neuropathic pain assessment: update on laboratory diagnostic tools. Curr Opin Anaesthesiol 2015;28:537-545. 
Truini A, Biasiotta A, Di Stefano G, et al. Does the epidermal nerve fibre density measured by skin biopsy in patients with peripheral neuropathies correlate with neuropathic pain? Pain 2014;155: 828-832.

89 Gibbons $\mathrm{CH}$, Griffin JW, Polydefkis M, et al. The utility of skin biopsy for prediction of progression in suspected small fiber neuropathy. Neurology 2006;66:256-258.

90 Jiang MS, Yuan Y, Gu ZX, Zhuang SL. Corneal confocal microscopy for assessment of diabetic peripheral neuropathy: a meta-analysis. Br J Ophthalmol 2016;100:9-14.

* This recent meta-analysis pooled results of 13 studies with a total of 1680 participants, assessing CCM in diabetic peripheral neuropathy (DPN). They found corneal nerve fiber density, nerve branch density and nerve fiber length to be significantly reduced in DPN patients compared to healthy controls.

91 Tavakoli M, Marshall A, Pitceathly R, et al. Corneal confocal microscopy: a novel means to detect nerve fibre damage in idiopathic small fibre neuropathy. Exp Neurol 2010;223:245-250.

92 Stettner M, Hinrichs L, Guthoff R, et al. Corneal confocal microscopy in chronic inflammatory demyelinating polyneuropathy. Ann Clin TransI Neurol 2016;3:88-100.

93 Pop-Busui R, Evans GW, Gerstein HC, et al. Effects of cardiac autonomic dysfunction on mortality risk in the Action to Control Cardiovascular Risk in Diabetes (ACCORD) trial. Diabetes Care 2010;33:1578-1584.

94 Sahuc $\mathrm{P}$, Chiche L, Dussol B, et al. Sudoscan as a noninvasive tool to assess sudomotor dysfunction in patients with Fabry disease: results from a case-control study. Ther Clin Risk Manag 2016;12:135-138.

* This prospective case-control study included 18 patients with Fabry disease (FD) and 18 matched controles. This is the first study to assess sudomotor function in FD using a sudoscan. Tests results are in accordance with QSART, but more rapid; little technical training is required and no calculations.

95 Yajnik CS, Kantikar V, Pande A, et al. Screening of cardiovascular autonomic neuropathy in patients with diabetes using non-invasive quick and simple assessment of sudomotor function. Diabetes Metab 2013; 39:126-131.

96 Hoeijmakers JG, Faber CG, Lauria G, et al. Small-fibre neuropathies--advances in diagnosis, pathophysiology and management. Nat Rev Neurol 2012;8:369-379.

97 Hoitsma E, Reulen JP, de Baets $M$, et al. Small fiber neuropathy: a common and important clinical disorder. J Neurol Sci 2004;227:119-130.

98 Imai E, Kaminaga T, Takada K, et al. Radioactive defect on I-123 MIBG myocardial SPECT imaging in a patient with cardiac sarcoidosis. Clin Nucl Med 2002;27:729-730.

99 Jamali HK, Waqar F, Gerson MC. Cardiac autonomic innervation. J Nucl Cardiol 2017; 24:1558-1570.

* A comprehensive review of the basic anatomy, physiology, and pathophysiology of the cardiac autonomic nervous system including adrenergic receptors.

100 Misumi I, Kimura Y, Hokamura Y, et al. Scintigraphic detection of regional disruption of the adrenergic nervous system in sarcoid heart disease. Jpn Circ J 1996;60:774-778.

101 Nagamachi S, Jinnouchi S, Nakahara H, et al. 123I-MIBG myocardial scintigraphy in diabetic patients: relationship to autonomic neuropathy. Nucl Med Commun 1996;17:621-632.

102 Sisson JC, Shapiro B, Meyers L, et al. Metaiodobenzylguanidine to map scintigraphically the adrenergic nervous system in man. J Nucl Med 1987;28:1625-1636.

103 van Kroonenburgh M, Mostard R, Voo S. Metaiodobenzylguanidine scintigraphy in pulmonary and cardiac disease. Curr Opin Pulm Med 2010;16:511-515.

104 Finnerup NB, Attal N, Haroutounian S, et al. Pharmacotherapy for neuropathic pain in adults: a systematic review and meta-analysis. Lancet Neurol 2015;14:162-173.

** An excellent systematic review and meta-analysis of pharmacotherapy for neuropathic pain, with number needed to treat and number needed to harm reported for each drug. Most positive trials show NNTs for $50 \%$ pain relief ranging from about 4 to 10 , emphasizing modest overall study outcomes in neuropathic pain.

105 Ho TW, Backonja M, Ma J, et al. Efficient assessment of neuropathic pain drugs in patients with small fiber sensory neuropathies. Pain 2009;141:19-24.

106 Moore RA, Derry S, Aldington D, et al. Amitriptyline for neuropathic pain in adults. Cochrane Database Syst Rev 2015;CD008242.

107 Attal N, Cruccu G, Baron R, et al. EFNS guidelines on the pharmacological treatment of neuropathic pain: 2010 revision. Eur J Neurol 2010;17:1113-e1188. 
108 Moore RA, Straube S, Wiffen PJ, et al. Pregabalin for acute and chronic pain in adults. Cochrane Database Syst Rev 2009;CD007076.

109 Moore RA, Wiffen PJ, Derry S, et al. Gabapentin for chronic neuropathic pain and fibromyalgia in adults. Cochrane Database Syst Rev 2014;CD007938.

110 Wiffen PJ, Derry S, Lunn MP, Moore RA. Topiramate for neuropathic pain and fibromyalgia in adults. Cochrane Database Syst Rev 2013; CD008314.

111 Wiffen PJ, Derry S, Moore RA. Lamotrigine for acute and chronic pain. Cochrane Database Syst Rev 2011;CD006044.

112 Wiffen PJ, Derry S, Moore RA, McQuay HJ. Carbamazepine for acute and chronic pain in adults. Cochrane Database Syst Rev 2011;CD005451.

113 Sommer C, Cruccu G. Topical treatment of peripheral neuropathic pain: applying the evidence. J Pain Symptom Manage 2017;53:614-629.

* Recent review of topical (lidocaine $5 \%$ and capsaicin $8 \%$ patch) treatment in peripheral neuropathic pain suggesting patients may benefit from the use of topical agents if the safety and tolerability of oral agents is a concern. However, more high-quality head-to-head randomized, controlled studies are needed.

114 Bauchy F, Mouraux A, Deumens R, et al. Feasibility of topical applications of natural high-concentration capsaicinoid solutions in patients with peripheral neuropathic pain: a retrospective analysis. Pain Res Manag 2016;2016:9703036.

115 Estanislao L, Carter K, McArthur J, et al. A randomized controlled trial of $5 \%$ lidocaine gel for HIVassociated distal symmetric polyneuropathy. J Acquir Immune Defic Syndr 2004;37:1584-1586.

116 Kieburtz K, Simpson D, Yiannoutsos C, et al. A randomized trial of amitriptyline and mexiletine for painful neuropathy in HIV infection. AIDS Clinical Trial Group 242 Protocol Team. Neurology 1998; 51:1682-1688.

117 Shlay JC, Chaloner K, Max MB, et al. Acupuncture and amitriptyline for pain due to HIV-related peripheral neuropathy: a randomized controlled trial. Terry Beirn Community Programs for Clinical Research on AIDS. JAMA 1998;280:1590-1595.

118 Simpson DM, Schifitto G, Clifford DB, et al. Pregabalin for painful HIV neuropathy: a randomized, double-blind, placebo-controlled trial. Neurology 2010;74:413-420.

119 Hammack JE, Michalak JC, Loprinzi CL, et al. Phase III evaluation of nortriptyline for alleviation of symptoms of cis-platinum-induced peripheral neuropathy. Pain 2002;98:195-203.

120 Kautio AL, Haanpaa M, Saarto T, Kalso E. Amitriptyline in the treatment of chemotherapy-induced neuropathic symptoms. J Pain Symptom Manage 2008;35:31-39.

121 Rao RD, Michalak JC, Sloan JA, et al. Efficacy of gabapentin in the management of chemotherapyinduced peripheral neuropathy: a phase 3 randomized, double-blind, placebo-controlled, crossover trial (N00C3). Cancer 2007;110:2110-2118.

122 Kizawa M, Mori K, lijima M, et al. Intravenous immunoglobulin treatment in painful sensory neuropathy without sensory ataxia associated with Sjogren's syndrome. J Neurol Neurosurg Psychiatry 2006; 77:967-969.

123 Molina JA, Benito-Leon J, Bermejo F, et al. Intravenous immunoglobulin therapy in sensory neuropathy associated with Sjogren's syndrome. J Neurol Neurosurg Psychiatry 1996;60:699.

124 Morozumi S, Kawagashira Y, lijima M, et al. Intravenous immunoglobulin treatment for painful sensory neuropathy associated with Sjogren's syndrome. J Neurol Sci 2009;279:57-61.

125 Parambil JG, Tavee JO, Zhou L, et al. Efficacy of intravenous immunoglobulin for small fiber neuropathy associated with sarcoidosis. Respir Med 2011;105:101-105.

126 Souayah N, Chin RL, Brannagan TH, et al. Effect of intravenous immunoglobulin on cerebellar ataxia and neuropathic pain associated with celiac disease. Eur J Neurol 2008;15:1300-1303.

127 Wakasugi D, Kato T, Gono T, et al. Extreme efficacy of intravenous immunoglobulin therapy for severe burning pain in a patient with small fiber neuropathy associated with primary Sjogren's syndrome. Mod Rheumatol 2009;19:437-440.

128 Wijnen PA, Cremers JP, Nelemans PJ, et al. Association of the TNF-alpha G-308A polymorphism with TNF-inhibitor response in sarcoidosis. Eur Respir J 2014;43:1730-1739. 
129 Tavee JO, Karwa K, Ahmed Z, et al. Sarcoidosis-associated small fiber neuropathy in a large cohort: clinical aspects and response to IVIG and anti-TNF alpha treatment. Respir Med 2017;125:135-138.

** A very interesting paper describing responses to IVIG and anti-TNF-alpha treatment in sarcoidosisassociated small fiber neuropathy. Among 115 patients, symptomatic improvement was seen in $75 \%$ with IVIG, in $67 \%$ with anti-TNF-alpha, and in $71 \%$ with both treatments.

130 Heij L, Niesters M, Swartjes M, et al. Safety and efficacy of ARA 290 in sarcoidosis patients with symptoms of small fiber neuropathy: a randomized, double-blind pilot study. Mol Med 2012;18: 1430-1436.

131 Culver DA, Dahan A, Bajorunas D, et al. Cibinetide improves corneal nerve fiber abundance in patients with sarcoidosis-associated small nerve fiber loss and neuropathic pain. Invest Ophthalmol Vis Sci 2017;58:BIO52-BIO60.

132 Gerson MC, Craft LL, McGuire N, et al. Carvedilol improves left ventricular function in heart failure patients with idiopathic dilated cardiomyopathy and a wide range of sympathetic nervous system function as measured by iodine 123 metaiodobenzylguanidine. J Nucl Cardiol 2002;9:608-615.

133 Smulders NM, Bast A, van Kroonenburgh MJ, Drent M. Improvement of cardiac sympathetic nerve function in sarcoidosis. Sarcoidosis Vasc Diffuse Lung Dis 2008;25:140-142.

134 Jankovic J, Gilden JL, Hiner BC, et al. Neurogenic orthostatic hypotension: a double-blind, placebocontrolled study with midodrine. Am J Med 1993;95:38-48.

135 Wright RA, Kaufmann HC, Perera R, et al. A double-blind, dose-response study of midodrine in neurogenic orthostatic hypotension. Neurology 1998;51:120-124.

136 Biaggioni I, Freeman R, Mathias CJ, et al. Randomized withdrawal study of patients with symptomatic neurogenic orthostatic hypotension responsive to droxidopa. Hypertension 2015;65:101-107.

137 Kaufmann H, Freeman R, Biaggioni I, et al. Droxidopa for neurogenic orthostatic hypotension: a randomized, placebo-controlled, phase 3 trial. Neurology 2014;83:328-335.

138 Vinik Al, Erbas T, Casellini CM. Diabetic cardiac autonomic neuropathy, inflammation and cardiovascular disease. J Diabetes Investig 2013;4:4-18.

139 Casanova-Molla J, Grau-Junyent JM, Morales M, Valls-Sole J. On the relationship between nociceptive evoked potentials and intraepidermal nerve fiber density in painful sensory polyneuropathies. Pain 2011;152:410-418. 



\section{Chapter}

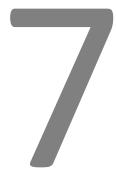

Determination of the smallest detectable change (SDC) and the minimal important difference (MID) for the Small Fiber Neuropathy Screening List (SFNSL) in sarcoidosis. Interpretability of the SFNSL

M. Voortman, E. Beekman, M. Drent, E. Hoitsma, J. De Vries Sarcoidosis Vasculitis and Diffuse Lung Diseases 2018;35(4):333-341 


\section{Abstract}

\section{Background}

Previous studies found that $40-60 \%$ of the sarcoidosis patients suffer from small fiber neuropathy (SFN), substantially affecting quality of life. SFN is difficult to diagnose, as a gold standard is still lacking. The need for an easily administered screening instrument to identify sarcoidosis-associated SFN symptoms led to the development of the SFN Screening List (SFNSL). The usefulness of any questionnaire in clinical management and research trials depends on its interpretability. Obtaining a clinically relevant change score on a questionnaire requires that the smallest detectable change (SDC) and minimal important difference (MID) are known. The aim of this study was to determine the SDC and MID for the SFNSL in patients with sarcoidosis.

\section{Methods}

Patients with neurosarcoidosis and/or sarcoidosis-associated SFN symptoms ( $N=138$ ) included in the online Dutch Neurosarcoidosis Registry participated in a prospective, longitudinal study. Anchor-based and distribution-based methods were used to estimate the MID and SDC, respectively.

\section{Results}

The SFNSL was completed both at baseline and at 6-months' follow-up by 89/138 patients. A marginal ROC curve (0.6) indicated cut-off values of 3.5 points, with $73 \%$ sensitivity and $49 \%$ specificity for change. The SDC was 11.8 points.

\section{Conclusion}

The MID on the SFNSL is 3.5 points for a clinically relevant change over a 6-month period. The MID can be used in the follow-up and management of SFN-associated symptoms in patients with sarcoidosis, though with some caution as the SDC was found to be higher. 


\section{Introduction}

Small fiber neuropathy (SFN) is a disabling generalized sensory nerve disorder with structural and functional abnormalities of small fibers. It is characterized histopathological by degeneration of small fiber nerve endings, selectively involving thin myelinated $\mathrm{A} \partial$ - and unmyelinated $\mathrm{C}$-fibers. ${ }^{1}$ Small fibers are associated with thermal and nociceptive sensations (somatic) and autonomic function, resulting in symptoms of neuropathic pain and autonomic dysfunction. ${ }^{2,3}$ The widespread spectrum of symptoms is associated with substantial negative effect on patients' quality of life (QoL). ${ }^{4,5}$

To date, SFN has been difficult to diagnose, as a gold standard is still lacking. The diagnosis is based on the presence of characteristic clinical features in combination with abnormalities on neurophysiological tests and/or reduced numbers of small fibers in skin biopsy. ${ }^{3,6-8}$

SFN can be an epiphenomenon in many diseases, ${ }^{9}$ including sarcoidosis. Sarcoidosis is a multisystem disorder that represents a burden on patients' lives. The clinical manifestation, natural history, and prognosis of sarcoidosis are highly variable, and its course is often unpredictable. ${ }^{10}$ In addition to specific organ-related symptoms with functional impairments, sarcoidosis patients have disabling non-specific symptoms, including fatigue, physical impairments, everyday cognitive failure, and pain. ${ }^{5,11}$ Previous studies found that $40-60 \%$ of the sarcoidosis patients suffer from SFN. ${ }^{12-15}$ However, there is as yet insufficient awareness of this among clinicians responsible for the follow-up of sarcoidosis patients. There is a clear need for an easily administered small fiber neuropathy (SFN) screening instrument to identify sarcoidosis-associated SFN in general clinical practice and for research purposes. To meet this need, we have previously developed the Small Fiber Neuropathy Screening List (SFNSL). ${ }^{16}$

The quality of a (screening) questionnaire is determined by its validity, reliability and responsiveness ${ }^{17,18}$ as well as interpretability. ${ }^{19}$ Interpretability is an important property of questionnaires that are regularly used in daily practice; it refers to what the SFNSL score means for the patient. ${ }^{19}$ Previously, Sun et al. showed the validity of the SFNSL in a different cohort, reporting a high correlation between intraepidermal nerve fiber density and the SFNSL, with a sensitivity of $94.1 \%$ and a specificity of $90.9 \%{ }^{20}$

For evaluative purposes, interpretability is assessed by change in scores: it is important to know when it can be said that a patient has improved or when their situation has worsened. Interpreting change in score requires two benchmarks: the measurement error, expressed as the smallest detectable change (SDC), and the minimal important difference (MID). The SDC represents the minimal change that a patient must show on the scale to ensure that the observed change is real and not just measurement error. ${ }^{19}$ 
The MID is the smallest change score that a patient perceives to be important. ${ }^{21}$ Establishing the SDC and MID will help clinicians interpret the clinical meaning of changes in SFNSL scores over time at individual level. At group level, both measures can be used to perform methodologically sound research and for the clinical interpretation of research findings. Several previous studies have used the SFNSL scores as an outcome measure to determine therapeutic response, and have found significant differences between placebo versus study drugs in patients suffering from SFN. ${ }^{22-24}$ Although these differences were statistically significant, they might not be relevant to a patient, since no MID and SDC were known for the SFNSL at the time. Till now, no SDC or MID has been established for this questionnaire in patients with sarcoidosis. It is important to determine changes in the SFN-related complaints, and knowledge is therefore needed regarding the measurement properties of the SFNSL.

The present study aimed to determine the measurement error (SDC) and interpretability (MID) of the Small Fiber Neuropathy Screening List SFNSL in patients with sarcoidosis.

\section{Methods}

\section{Study design and participants}

A prospective cohort study was performed. From June 2014 until June 2017, a Dutch national sample of neurosarcoidosis and/or sarcoidosis patients with SFN-associated symptoms was recruited by inviting these patients to join an online neurosarcoidosis registry, the Dutch Neurosarcoidosis Registry (www.neurosarcoidose.nl). This registry includes patients with a recent diagnosis of neurosarcoidosis and/or SFN-associated symptoms as well as patients whose disease was of longer duration. The diagnosis of neurosarcoidosis and/or SFN-associated symptoms was confirmed by a neurologist for all patients. The diagnosis neurosarcoidosis was established using the Zajicek or Marangoni (modified Zajicek) criteria, labelling patients as possible, probable or definite neurosarcoidosis. ${ }^{25,26} \mathrm{SFN}$ is considered a 'paraneurosarcoidosis', since it is not associated with the hallmark of sarcoidosis, granuloma formation, in the small fibers. ${ }^{3,27-29}$ The Dutch Neurosarcoidosis Registry is an ongoing registry recording demographic parameters, disease-related parameters and multiple electronic questionnaires.

All patients were $>18$ years old, had sufficient command of the Dutch language and had access to the internet. Patients included in the Dutch Neurosarcoidosis Registry agreed to participate in online research studies. The registry and study protocols have been 
approved by the Medical Ethics Committee of the St. Antonius Hospital Nieuwegein. Digital informed consent was obtained from all patients.

\section{Procedure}

Curavista (a certified eHealth platform) provided the online platform for this study and maintained the website. The data were collected online by Curavista and were exported to a database.

Invitation to complete demographic questions and questionnaires were sent by email via the online Dutch Neurosarcoidosis Registry to all patients every 6 months. The following demographic and medical characteristics of patients were collected (selfreported): sex, age, time since diagnosis of sarcoidosis, medications use, use of prednisone, use of azathioprine (Imuran), use of methotrexate, use of infliximab (Remicade), use of adalimumab (Humira), use of cyclophosphamide and use of other medication. Patients received usual care and medication for their sarcoidosis treatment. SFN-associated symptoms are likely to remain stable or progress in time, since no curative treatment is as yet available. At baseline, the whole cohort was asked

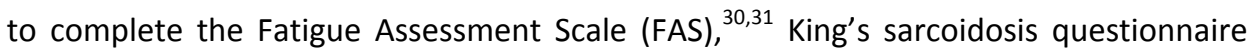
(KSQ), ${ }^{32,33}$ World Health Organization Quality of Life (WHOQOL) Bref ${ }^{34}$ and a visual analogue scale (VAS) for pain. ${ }^{35}$ Furthermore, they were asked to complete the SFNSL at baseline (t0) and at 6-month follow-up (t1). An anchor question was added at $\mathrm{t} 1$.

\section{Outcome measures}

\section{Small Fiber Neuropathy Screening List}

The SFNSL is a 21-item self-administered questionnaire to screen for symptoms related to SFN. The response scale is a five-point scale (0 never/not to 4 always/severe); scores on the SFNSL can range from 0 to 84 (see Appendix). Change scores can range from -84 to 84 between t0 (baseline) and t1 (6-month follow-up). Change scores for the SFNSL were calculated by subtracting the baseline score from the 6-month score. A positive change on the SFNSL indicates an increase in SFN-related symptoms.

Cut-off values for diagnosing SFN using the SFNSL have been established after crossvalidation in a second group based on temperature threshold testing (TTT). ${ }^{16}$ The cutoff score for the SFNSL is 11: a score below 11 indicates few or no SFN-related symptoms (normal TTT), while a score of 11-48 indicates probable or highly likely SFN, and a score above 48 is indicative of SFN (abnormal TTT). ${ }^{16}$

The questionnaire is divided into two parts; part 1 assesses how often patients experience specific complaints, and part 2 assesses how severely patients experience 
specific complaints. Patients are instructed to answer each question, even if they do not have that specific complaint. No score was generated for patients who did not fully complete the questionnaire. The PDF and digital version of the English SFNSL can be found at http://www.wasog.org/education-research/questionnaires.html, as well as Danish, Dutch, French, German, Italian and Japanese versions (Cild care foundation: www.ildcare.nl).

\section{Anchor question}

The anchor question asked at $\mathrm{t} 1$ was: Compared to six months ago, do you think that - regarding the questions about SFN complaints (SFNSL) - you are doing worse, the same or better?

\section{Statistical analysis}

Descriptive variables were calculated as means ( \pm standard deviation, SD) or medians (25th-75th percentiles) for the continuous variables, depending on the data distribution, and as percentages for the dichotomous variables. Data of all subjects were checked for missing values, distribution (skewness and excess kurtosis), and outliers. Patients providing insufficient information on the SFNSL at t1 or who did not answer the anchor question were excluded from the analysis using case-wise deletion. Following Crosby et al. ${ }^{36}$ and Revicki et al., ${ }^{37}$ anchor-based and distribution-based approaches were used to establish the MID and SDC, respectively. The anchor-based approach was used with an external criterion, or anchor, represented by individual patients' perceived change over time. In a distribution-based approach, the distributional characteristics of our study sample were used to calculate the standard error of measurement (SEM), which was used to determine the SDC. Following De Vet et al. ${ }^{38}$ and van Kampen et al., ${ }^{19}$ the MID itself was not calculated using effect sizes or the SEM (i.e. data variability). Instead, the SDC was determined, thus taking into account the variation in the scores on the SFNSL scale due to measurement error. The MID was determined by the anchor-based method only. The MID is also known as the minimal important change (MIC) or the minimal clinically important difference (MCID), but strictly spoken these are not the same. ${ }^{39-41}$ The MID addressed in this paper implies the longitudinal within-person change in scores.

A minimum of 50 patients is considered sufficient for assessing measurement properties. ${ }^{42}$ Since the risk of loss to follow-up often increases after several months, we wanted to include at least 100 patients at baseline. Patients who only completed the t0 assessment were compared with patients who also completed the t 1 assessment as regards sex, age, medications use, and the score on the SFNSL at t0, using independent 
t-tests and chi-square tests. At a minimal statistical power of $80 \%, \mathrm{p}$ values below 0.05 were considered significant.

\section{Smallest detectable change (measurement error)}

Data from t0 were used to determine the measurement error. The change score distribution was tested for normality (skewness and excess kurtosis), as it was important that the change scores should be normally distributed and close to zero. ${ }^{43}$ Measurement error can be expressed as the standard error of measurement (SEM) or as SDC. ${ }^{19}$ The SEM represents the standard deviation of repeated measures in one patient, and was calculated using the intraclass correlation coefficient (ICC): SEM = SD * $V\left(1-I C C_{\text {agreement }}\right) .^{21}$ The ICC was calculated using a two-way mixed effects model for absolute agreement. The SDC, the smallest change in a score that you can detect with the SFNSL above measurement error, ${ }^{19}$ is also known as the minimal detectable change when using its $95 \%$ confidence interval $\left(\mathrm{MDC}_{95}\right)$, and was calculated using the SEM: $1.96 \times \sqrt{ } 2 \times$ SEM $^{44}$ These values were expressed in the unit of measurement of the SFNSL scale.

\section{Minimal important change}

In the anchor-based approach, a receiver operating characteristic (ROC) curve was used to estimate the MID, ${ }^{38}$ the smallest measured change in score that is perceived as being relevant by the patients. The anchor question was considered the gold standard to establish a cut-off value for the SFNSL. The anchor question firstly distinguished patients who had improved or worsened from patients who had remained stable, and secondly, distinguished improved patients from not improved (stable or worsened) patients in terms of the change in complaints they had perceived. The ROC curve was obtained by plotting the sensitivity against 1-specificity for each possible SFNSL change score. The ROC curve was used to estimate the anchor-based MID for the SFNSL. Most important for clinical practices is the MID for a change in score. For research purposes, the MID for improvement is preferable, therefore we have also added this MID. The area under the ROC curve represents the probability that the SFNSL will discriminate between two patient states, referred to as 'important change (improved/worsened)' and 'no important change (i.e. the same)'. An area under the curve between 0.5-0.6 was regarded as 'failed', between 0.6-0.7 as 'poor', between 0.7-0.8 as 'fair' and between 0.8-0.9 as 'good'. The optimal cut-off point was considered to be the lowest value for which the sum of percentages of true positive and true negative classifications was largest, assuming that sensitivity (ruling out important change and improvement on the SFNSL) and specificity (ruling in important change and improvement on the 
SFNSL) are equally important for this patient population. This optimal cut-off point was used to establish the overall MID.

Change scores on the SFNSL were calculated as each patient's t1 score (6-month followup) minus their t0 score (baseline). This was done for each subscale, based on the anchor question. The MID for deterioration was defined as the mean change score in the subgroup that worsened (i.e. perceived their complaints as 'worse' after six months), while the MID for improvement was defined as the mean change score in the subgroup that improved (i.e. perceived their complaints as 'better' after six months).

\section{Results}

The flowchart of the study is displayed in Figure 7.1. A total of 150 patients subscribed to the online Dutch Neurosarcoidosis Registry study. Twelve of them never completed the SFNSL and were therefore excluded from the analysis. The response rate at t0 (baseline), with 138 respondents, was $92 \%$. Of these 138 patients, 89 (64\%) completed the SFNSL and the anchor question at $\mathrm{t} 1$, and could thus be analyzed.

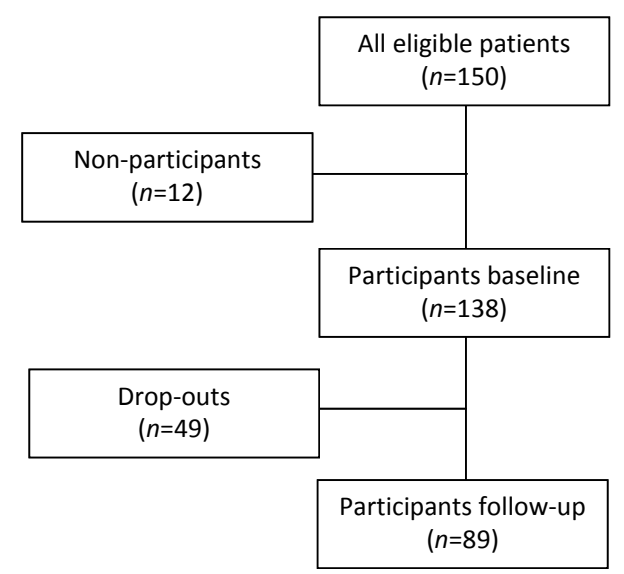

Figure 7.1 Flowchart of study patients.

Demographical and medical characteristics of the 89 patients are presented in Table 7.1. Neurosarcoidosis was established in 44 patients (49.4\%) and SFN-associated complaints in 81 patients (91\%). The organ involvement presented in Table 7.1 was selfreported by completing the $\mathrm{KSQ}$. 
The mean scores on the FAS, SFNSL and VAS pain were 32.1, 34.9 and 4.4, respectively (see Tables 7.1 and 7.2). The VAS correlates well with the SFNSL score $(R=0.542$; p-value $\leq 0.0001)$.

Table 7.1 Demographic and medical characteristics of neurosarcoidosis patients and/or patients suffering from SFN symptoms $(n=89)$.

\begin{tabular}{|c|c|}
\hline Characteristics & $\begin{array}{c}\text { Number }(\%) \\
\text { or median }\left(25^{\text {th }}-75^{\text {th }} \text { percentile }\right) \\
\text { or mean } \pm \text { SD }\end{array}$ \\
\hline \multicolumn{2}{|l|}{ demographics } \\
\hline sex: male & $45(51)$ \\
\hline age $(y r)$ & $53.2 \pm 10.5$ \\
\hline \multicolumn{2}{|l|}{ medical Variables } \\
\hline time since diagnosis $(y r)$ & $6(2-12)$ \\
\hline age at diagnosis of neurosarcoidosis (yr) & $44(49.4)$ \\
\hline SFN-related complaints (SFNSL >11) & $81(91)$ \\
\hline medication use & $62(70)$ \\
\hline prednisone & $32(36)$ \\
\hline azathioprine $\left(\operatorname{Imuran}^{\circledR}\right)$ & $3(3)$ \\
\hline methotrexate & $30(34)$ \\
\hline infliximab (Remicade ${ }^{\circledR}$ ) & $12(14)$ \\
\hline adalimumab (Humira ${ }^{\circledR}$ ) & $4(5)$ \\
\hline cyclophosphamide & $0(0)$ \\
\hline other medication & $14(16)$ \\
\hline \multicolumn{2}{|l|}{ psychological variables } \\
\hline FAS score & $32.1 \pm 8.3$ \\
\hline \multicolumn{2}{|l|}{ WHOQOL-BREF } \\
\hline physical health & $10.5 \pm 2.7$ \\
\hline psychological health & $13.2 \pm 2.7$ \\
\hline social relationships & $13.5 \pm 2.9$ \\
\hline environment & $15.2 \pm 2.2$ \\
\hline \multicolumn{2}{|l|}{$\mathrm{KSQ}$} \\
\hline general health status & $55 \pm 19.2$ \\
\hline lung $(n=60, \%)$ & $60.9 \pm 23.8$ \\
\hline skin $(n=20)$ & $66.7 \pm 22.5$ \\
\hline eyes $(n=41)$ & $54.3 \pm 22.6$ \\
\hline medication $(n=62)$ & $65.7 \pm 26.7$ \\
\hline
\end{tabular}

$\mathrm{SFN}=$ small fiber neuropathy; SFNSL=small fiber neuropathy screening list; FAS=fatigue assessment scale; WHOQOL-BREF=World Health Organization Quality of Life BREF; KSQ=King's sarcoidosis questionnaire. 
Table 7.2 Small Fiber Neuropathy Screening List (SFNSL) and VAS pain scores $(n=89)$.

\begin{tabular}{lc}
\hline & $\begin{array}{c}\text { Mean } \pm \text { SD } \\
\text { or number }(\%)\end{array}$ \\
\hline SFNSL t0 score (0-84) & $34.9 \pm 18.7$ \\
SFNSL t0, definitely no SFN $(<11)$ & $8(9)$ \\
SFNSL t0, probable SFN $(11-48)$ & $56(63)$ \\
SFNSL t0, definitely SFN $(>48)$ & $25(28)$ \\
VAS pain score & $4.4 \pm 2.8$ \\
\hline
\end{tabular}

VAS=visual analogue scale.

A statistically significantly higher age was found in the follow-up group compared to the age in the drop-out group $(t(136)=2.75, p<0.01)$. No other differences were found in the variables mentioned in table 7.1 nor the SFNSL at to between the patients who dropped-out of the study before $\mathrm{t} 1$ (i.e. the 6-month follow-up) and the patients remaining in the study.

\section{Smallest detectable change (measurement error)}

The $t 1$ questionnaires were completed within an average of 5.9 months (SD=0.57) after t0, without skewness but with a leptokurtic distribution (z-score>3.29), implying a higher peak and thus more clustered data around the mean. The change scores of the SFNSL were normally distributed (no statistically significant skewness or excess kurtosis) and close to zero, with an average of -2.44 (SD=8.12). The intraclass correlation coefficient ( ICC $_{\text {agreement }}$ ) was 0.95 for the SFNSL, with a SEM of 4.26 as calculated by the abovementioned equation. The calculated value of SDC or $\mathrm{MDC}_{95}$ was 11.8 .

\section{Minimal important difference}

The area under the ROC curve for importantly changed status was 0.6 , so the ability of the SFNSL to distinguish patients who worsened or improved from patients who remained stable was just above change level. The cut-off value for a MID in the SFNSL score was 3.5 points, corresponding to a sensitivity of $73 \%$ and a specificity of $49 \%: 73 \%$ of the patients were correctly identified as changed and $49 \%$ of the patients were correctly identified as stable (Figure 7.2a).

The area under the ROC curve for importantly improved status was also 0.6 . The cut off value for a MID in the SFNSL score was 8.5 points, corresponding with a sensitivity of $56 \%$ and a specificity of $78 \%: 56 \%$ of the patients were correctly identified as improved and $78 \%$ of the patients were correctly identified as not improved (Figure 7.2b). 

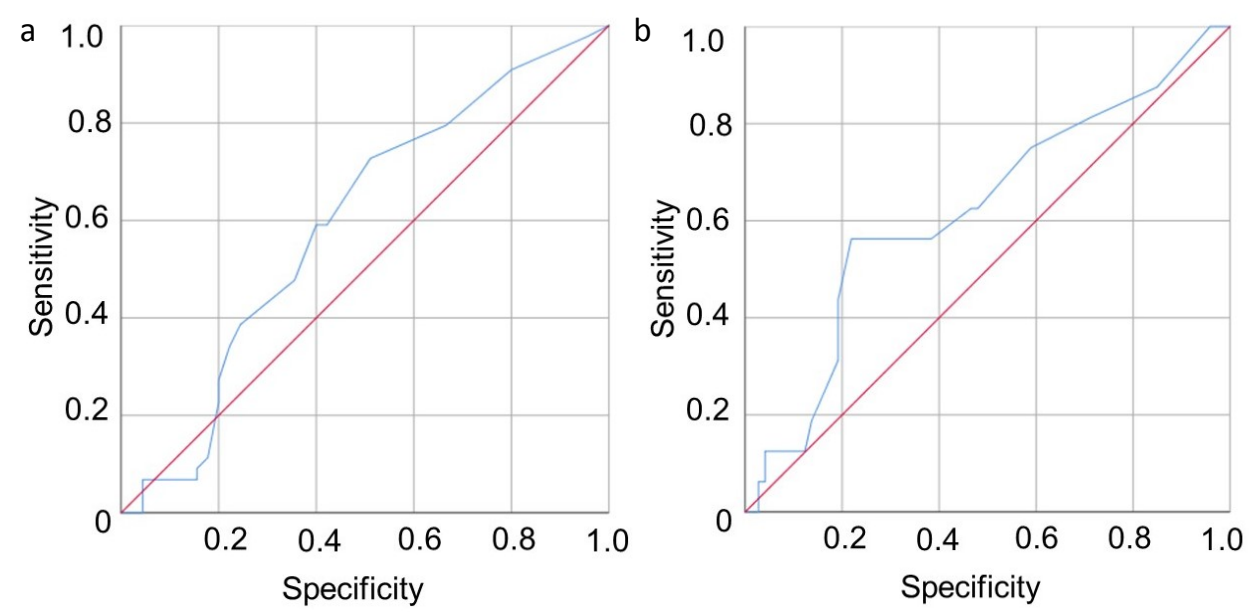

Figure 7.2 ROC curves comparing Small Fiber Neuropathy Screening List (SFNSL) with the anchor question. Diagonal segments are produced by ties. a) Patients with an importantly changed status versus patients with a stable status; b) Patients with an importantly improved status versus patients with a non-improved status.

The mean change scores on the SFNSL per subgroup based on the anchor question are presented in Table 7.3. When using these data, a MID of 0.14 for deterioration and a MID of 7.94 for improvement was found.

Table 7.3 Mean change scores on the SFNSL compared with those on the anchor question ( $n=89)$.

\begin{tabular}{lcc}
\hline & Anchor (perceived change in complaints) & Mean change score on SFNSL \\
& $n(\%)$ & mean \pm SD \\
\hline worse & $28(31)$ & $0.14 \pm 7.46$ \\
the same & $45(51)$ & $-2.09 \pm 8.47$ \\
better & $16(18)$ & $-7.94 \pm 5.62$ \\
\hline
\end{tabular}

\section{Discussion}

The SFNSL is a validated questionnaire to assess the presence of SFN-related symptoms in sarcoidosis. The present study established the SDC and MID for the SFNSL in a group of sarcoidosis patients. A MID of 3.5 was found to distinguish between change of complaints and no change of complaints.

To avoid any misconception, the SFNSL was developed to assess symptoms which might be related to SFN. The results might offer a clinician a first suspicion of SFN and stimulate him or her to refer this patient to a neurologist for further evaluation of the 
problem related to or probably associated with the symptoms. Even when the symptoms are not related to SFN they cause a substantial burden for the patients and have impact on their QOL. It is always hard to measure and follow-up non organ related symptoms. Moreover, so far no gold standard for diagnosing SFN exists. Therefore, the MID was calculated just to evaluate whether from the patient's perspective the symptoms changed or not. Hence, it is not meant to assess improvement of the underlying cause.

The MID is the smallest change score that patients perceive to be important. ${ }^{21}$ As such, it is the smallest change score that one wants to detect with the SFNSL. The SDC is the smallest change score that one can detect with the SFNSL in individual patients. The SDC we found was larger than the MID for detecting change of complaints and slightly larger than the MID for detecting improvement. This means that it is more difficult to distinguish a clinically important change from the measurement error with a large degree of certainty in individual cases, compared to patient-related outcomes (PROMs), where the SDC is smaller than the MID. ${ }^{19,45}$ In this case: if the individual change in score is 3.5 points or higher, there may be a clinical relevant change on the SFNSL. However, it may also be due to a measurement error on the scale. A change of 11.8 points or higher gives a high degree of certainty that it indicates a clinically relevant change in SFN related symptoms. Nevertheless, the similar results for both methods (MID for improvement 8.5 vs 8.0 ) might suggest that our estimation for MID is robust.

SFN is a major concern in sarcoidosis, leading to decreased QoL. ${ }^{4}$ SFN complaints are difficult to manage, since no curative treatment currently exists. ${ }^{3}$ The treatment of SFN is at present merely symptomatic (neuropathic pain medications) with poor to moderate responses. The validated SFNSL is a practical clinical screening tool that can guide clinicians in assessing whether patients are suffering from SFN-associated symptoms, but the interpretability of change scores (MID) over time was unclear. The MID is of great clinical importance, since it will help to manage these patients better. In clinical practice, a positive change in symptoms scores is not to be expected for many patients. This is why we decided to determine the MID, in order to enable doctors in clinical practice to distinguish changes in complaints.

For research purposes, we also determined the MID for improvement in SFNSL scores. The higher SDC is less relevant for use in clinical trials at group level than it is for use in individual patients. In clinical research, it is important to look at clinically relevant improvements of SFN complaints and not merely at a statistically significant change. The MID could therefore be used as a useful outcome measure in clinical trials. TNF- $\alpha$ inhibition and immunoglobulins have been reported to be effective treatment options for sarcoidosis-associated SFN. ${ }^{46-49}$ Whether these expensive treatments should be initiated for SFN is unclear. These treatment modalities, as well as new promising drugs 
such as Cibinetide ${ }^{\circledR}$ (ARA290) $)^{24,50}$ also need to be evaluated using the SFNSL and MID, to assess whether there is a clinically relevant effect, rather than a mere statistically significant increase in the number of small fibers (Cibinetide ${ }^{\circledR}$ ).

Our group has previously determined the MID for another validated questionnaire in sarcoidosis, the fatigue assessment scale (FAS). ${ }^{31}$ Fatigue is also a major concern in sarcoidosis, and determining the MID for the FAS has made a significant contribution to clinical practice in terms of improving the clinical interpretation of fatigue scores. The present study used the same methods (anchor-based and distribution-based methods). The area under the ROC curve (AUC) for both analyses (improvement and change in complaints on the SFNSL) was 0.6, which is poor. The low AUC can be explained, amongst other things, by the heterogeneity of sarcoidosis and the wide organ- as well as non-organ related symptoms. For instance, the MCID of the FAS has an AUC of 0.6 and the MCID of the VAS pain was $0.7 . .^{31,51}$ Therefore, an AUC of 0.6 cannot be considered a poor performance and may be used in clinical practice, especially since no other estimate is available.

This study had some limitations. One limitation may be that the patients were recruited using the online neurosarcoidosis registry, which includes neurosarcoidosis patients and patients suffering from SFN. Online registries may generate biases. However, of all patients, additional medical information was provided by their caregivers, confirming the diagnosis sarcoidosis. In addition, we examined symptoms that can only be assessed by asking the patients themselves. Moreover, it could also be argued that only patients who are motivated to participate in research studies registered for our online neurosarcoidosis study. This may have caused selection bias. Nevertheless, in our experience, all sarcoidosis patients are highly motivated to participate in studies.

Furthermore, we have used one anchor question to examine the MID. This has several limitations, but is in line with many other studies. ${ }^{31,51}$ It is an option to examine more anchors, but the disadvantage is that it may yield a different result, which raises the question what result than represents the better information. Therefore, we have chosen one simple anchor. We would like to acknowledge that a simple anchor is also previously used in other studies regarding questionnaires. ${ }^{52}$ Another limitation could be a recall bias. Patients were asked the anchor question after 6 months. However, neurologic complaints usually persist for quite some time and often do not improve or recover. Moreover, these complaints are continuously bothering the patient, so in our experience patients are capable to tell whether or not the complaints have worsened or not, even after 6 months. Furthermore, in the literature 6 months is a very common period when establishing a MID. ${ }^{39}$ 


\section{Conclusion}

The present study showed that a change of at least 3.5 points in the patients' SFNSL score represents a clinically important difference, in that the SFN-related symptoms showed a relevant increase or decrease. Some caution is warranted for the use of the MID in individual cases in the clinic, as the SDC was found to be higher than the MID. 


\section{References}

1 Lauria G, Lombardi R. Small fiber neuropathy: is skin biopsy the holy grail? Curr Diab Rep 2012;12: 384-392.

2 Themistocleous AC, Ramirez JD, Serra J, Bennett DL. The clinical approach to small fibre neuropathy and painful channelopathy. Pract Neurol 2014;14:368-379.

3 Voortman M, Fritz D, Vogels OJM, et al. Small fiber neuropathy: a disabling and underrecognized syndrome. Curr Opin Pulm Med 2017;23:447-457.

4 Bakkers M, Faber CG, Hoeijmakers JG, et al. Small fibers, large impact: quality of life in small-fiber neuropathy. Muscle Nerve 2014; 49:329-336.

5 Drent M, Strookappe B, Hoitsma E, De Vries J. Consequences of sarcoidosis. Clin Chest Med 2015; 36:727-737.

6 Blackmore D, Siddiqi ZA. Diagnostic criteria for small fiber neuropathy. J Clin Neuromuscul Dis 2017; 18:125-131.

7 Devigili G, Tugnoli V, Penza P, et al. The diagnostic criteria for small fibre neuropathy: from symptoms to neuropathology. Brain 2008;131:1912-1925.

8 Tesfaye S, Boulton AJ, Dyck PJ, et al. Diabetic neuropathies: update on definitions, diagnostic criteria, estimation of severity, and treatments. Diabetes Care 2010;33:2285-2293.

9 Bednarik J, Vlckova-Moravcova E, Bursova S, et al. Etiology of small-fiber neuropathy. J Peripher Nerv Syst 2009;14:177-183.

10 Statement on sarcoidosis. Joint Statement of the American Thoracic Society (ATS), the European Respiratory Society (ERS) and the World Association of Sarcoidosis and Other Granulomatous Disorders (WASOG) adopted by the ATS Board of Directors and by the ERS Executive Committee, February 1999. Am J Respir Crit Care Med 1999;160:736-755.

11 Hendriks C, Drent M, De Kleijn W, et al. Everyday cognitive failure and depressive symptoms predict fatigue in sarcoidosis: a prospective follow-up study. Respir Med 2018;138S:S24-S30.

12 Bakkers M, Merkies IS, Lauria G, et al. Intraepidermal nerve fiber density and its application in sarcoidosis. Neurology 2009;73:1142-1148.

13 Hoitsma $E$, Drent $M$, Verstraete $E$, et al. Abnormal warm and cold sensation thresholds suggestive of small-fibre neuropathy in sarcoidosis. Clin Neurophysiol 2003;114:2326-2333.

14 Hoitsma E, Marziniak M, Faber CG, et al. Small fibre neuropathy in sarcoidosis. Lancet 2002;359: 2085-2086.

15 Hoitsma E, Reulen JP, de Baets $M$, et al. Small fiber neuropathy: a common and important clinical disorder. J Neurol Sci 2004;227:119-130.

16 Hoitsma E, De Vries J, Drent M. The small fiber neuropathy screening list: construction and crossvalidation in sarcoidosis. Respir Med 2011;105:95-100.

17 Mokkink LB, Terwee CB, Patrick DL, et al. The COSMIN checklist for assessing the methodological quality of studies on measurement properties of health status measurement instruments: an international Delphi study. Qual Life Res 2010;19:539-549.

18 Mokkink LB, Terwee CB, Knol DL, et al. Protocol of the COSMIN study: COnsensus-based Standards for the selection of health Measurement INstruments. BMC Med Res Methodol 2006;6:2.

19 van Kampen DA, Willems WJ, van Beers LW, et al. Determination and comparison of the smallest detectable change (SDC) and the minimal important change (MIC) of four-shoulder patient-reported outcome measures (PROMs). J Orthop Surg Res 2013;8:40.

20 Sun B, Li Y, Liu L, et al. SFN-SIQ, SFNSL and skin biopsy of 55 cases with small fibre involvement. Int J Neurosci 2017;1-7.

21 de Vet HCW, Tewee CB, Mokkink LB, Knol DL. Measurement in medicine. New York: Cambridge University Press; 2011.

22 van Velzen M, Heij L, Niesters M, et al. ARA 290 for treatment of small fiber neuropathy in sarcoidosis. Expert Opin Investig Drugs 2014;23:541-550. 
23 Heij L, Niesters M, Swartjes M, et al. Safety and efficacy of ARA 290 in sarcoidosis patients with symptoms of small fiber neuropathy: a randomized, double-blind pilot study. Mol Med 2012;18: 1430-1436.

24 Dahan A, Dunne A, Swartjes M, et al. ARA 290 improves symptoms in patients with sarcoidosisassociated small nerve fiber loss and increases corneal nerve fiber density. Mol Med 2013;19:334-345.

25 Marangoni S, Argentiero V, Tavolato B. Neurosarcoidosis. Clinical description of 7 cases with a proposal for a new diagnostic strategy. J Neurol 2006;253:488-495.

26 Zajicek JP, Scolding NJ, Foster O, et al. Central nervous system sarcoidosis--diagnosis and management. QJM 1999;92:103-117.

27 Tavee J, Culver D. Sarcoidosis and small-fiber neuropathy. Curr Pain Headache Rep 2011; 15:201-206.

28 Culver DA, Ribeiro Neto ML, Moss BP, Willis MA. Neurosarcoidosis. Semin Respir Crit Care Med 2017; 38:499-513.

29 Datema M, Tannemaat MR, Drent M, Hoitsma E. [Neurosarcoidosis and paraneurosarcoidosis: new online registration of patients]. Ned Tijdschr Geneeskd 2015;159:A8383.

30 De Vries J, Michielsen $\mathrm{H}$, Van Heck GL, Drent $M$. Measuring fatigue in sarcoidosis: the Fatigue Assessment Scale (FAS). Br J Health Psychol 2004;9:279-291.

31 de Kleijn WP, De Vries J, Wijnen PA, Drent M. Minimal (clinically) important differences for the Fatigue Assessment Scale in sarcoidosis. Respir Med 2011;105:1388-1395.

32 Van Manen MJ, Wapenaar M, Strookappe B, et al. Validation of the King's Sarcoidosis Questionnaire (KSQ) in a Dutch sarcoidosis population. Sarcoidosis Vasc Diffuse Lung Dis 2016;33:75-82.

33 Patel AS, Siegert RJ, Creamer D, et al. The development and validation of the King's Sarcoidosis Questionnaire for the assessment of health status. Thorax 2013;68:57-65.

34 The World Health Organization Quality of Life Assessment (WHOQOL): development and general psychometric properties. Soc Sci Med 1998;46:1569-1585.

35 Williamson A, Hoggart B. Pain: a review of three commonly used pain rating scales. J Clin Nurs 2005; 14:798-804.

36 Crosby RD, Kolotkin RL, Williams GR. Defining clinically meaningful change in health-related quality of life. J Clin Epidemiol 2003;56:395-407.

37 Revicki D, Hays RD, Cella D, Sloan J. Recommended methods for determining responsiveness and minimally important differences for patient-reported outcomes. J Clin Epidemiol 2008;61:102-109.

38 de Vet HC, Ostelo RW, Terwee $\mathrm{CB}$, et al. Minimally important change determined by a visual method integrating an anchor-based and a distribution-based approach. Qual Life Res 2007;16:131-142.

39 Coster MC, Nilsdotter A, Brudin L, Bremander A. Minimally important change, measurement error, and responsiveness for the self-reported foot and ankle score. Acta Orthop 2017;88:300-304.

40 Jaeschke R, Singer J, Guyatt GH. Measurement of health status. Ascertaining the minimal clinically important difference. Control Clin Trials 1989;10:407-415.

41 Nordin A, Taft C, Lundgren-Nilsson A, Dencker A. Minimal important differences for fatigue patient reported outcome measures-a systematic review. BMC Med Res Methodol 2016;16:62.

42 Terwee CB, Mokkink LB, Knol DL, et al. Rating the methodological quality in systematic reviews of studies on measurement properties: a scoring system for the COSMIN checklist. Qual Life Res 2012; 21:651-657.

43 Stratford PW, Riddle DL. When minimal detectable change exceeds a diagnostic test-based threshold change value for an outcome measure: resolving the conflict. Phys Ther 2012;92:1338-1347.

44 Bland JM, Altman DG. Statistical methods for assessing agreement between two methods of clinical measurement. Lancet 1986;1:307-310.

45 Terwee CB, Roorda LD, Knol DL, et al. Linking measurement error to minimal important change of patient-reported outcomes. J Clin Epidemiol 2009;62:1062-1067.

46 Tavee JO, Karwa K, Ahmed Z, et al. Sarcoidosis-associated small fiber neuropathy in a large cohort: clinical aspects and response to IVIG and anti-TNF alpha treatment. Respir Med 2017.

47 Wijnen PA, Cremers JP, Nelemans PJ, et al. Association of the TNF-alpha G-308A polymorphism with TNF-inhibitor response in sarcoidosis. Eur Respir J 2014;43:1730-1739.

48 Parambil JG, Tavee JO, Zhou L, et al. Efficacy of intravenous immunoglobulin for small fiber neuropathy associated with sarcoidosis. Respir Med 2011;105:101-105. 
49 Saito H, Yamaguchi T, Adachi Y, et al. Neurological symptoms of sarcoidosis-induced small fiber neuropathy effectively relieved with high-dose steroid pulse therapy. Intern Med 2015;54:1281-1286.

50 Culver DA, Dahan A, Bajorunas D, et al. Cibinetide improves corneal nerve fiber abundance in patients with sarcoidosis-associated small nerve fiber loss and neuropathic pain. Invest Ophthalmol Vis Sci 2017;58:BIO52-BIO60.

51 Ostelo RW, Deyo RA, Stratford P, et al. Interpreting change scores for pain and functional status in low back pain: towards international consensus regarding minimal important change. Spine (Phila Pa 1976) 2008;33:90-94.

52 Iordens GIT, Den Hartog D, Tuinebreijer WE, et al. Minimal important change and other measurement properties of the Oxford elbow score and the quick disabilities of the arm, shoulder, and hand in patients with a simple elbow dislocation; validation study alongside the multicenter FuncSiE trial. PLoS One 2017;12:e0182557. 



\section{Chapter 8}

\section{The burden of sarcoidosis symptoms from}

a patient perspective

M. Voortman, C.M.R. Hendriks, M.D.P. Elfferich, F. Bonella, J. Møller, J. De Vries, U. Costabel, M. Drent Lung 2019. Apr;197(2):155-161 


\section{Abstract}

\section{Background}

The clinical manifestations of sarcoidosis vary widely, depending on the intensity of the inflammation and the organ systems affected. Hence, sarcoidosis patients may suffer from a great variety of symptoms. The aim of this study was to compare the selfreported burden of sarcoidosis patients in Denmark, Germany and the Netherlands, especially the prevalence of fatigue and small fiber neuropathy (SFN)-related symptoms, as well as differences in treatment strategies.

\section{Methods}

A cross-sectional web-based anonymous survey about complaints was conducted among sarcoidosis patients. Patients were invited to take part through the sarcoidosis patient societies as well as through outpatient sarcoidosis clinics in these countries.

\section{Results}

The questionnaire was completed by 1072 sarcoidosis patients (152 Danish, 532 German and 388 Dutch). Almost all patients reported having sarcoidosis-associated symptoms (organ-related as well as non-specific, non-organ related). Fatigue was reported by almost all respondents (90\%), followed by pulmonary symptoms $(72.4 \%)$. More than $50 \%$ of the respondents were being treated with prednisone, which was comparable in all 3 countries. In contrast, second- and third-line treatment differed substantially between Denmark, Germany and the Netherlands.

\section{Conclusion}

Sarcoidosis patients in Denmark, Germany and the Netherlands present with similar self-reported symptoms, organ-related as well as non-specific, non-organ related. Fatigue (90\%) and symptoms associated with SFN (86\%) were highly prevalent in all 3 countries. 


\section{Introduction}

Sarcoidosis occurs throughout the world, affecting all races and ages. Its' true prevalence remains unknown. To date, studies based on nationwide registries of demographic factors and diagnoses are relatively old and scarce. ${ }^{1-4}$ Moreover, the epidemiological assessment of sarcoidosis and its manifestations is problematic due to lack of consistent case definition, lack of sensitivity and specificity of diagnostic tests, variable diagnostic intensity and variable diagnostic methods. ${ }^{5}$

The clinical manifestation, natural history and prognosis of sarcoidosis are highly variable, and its course is often unpredictable. ${ }^{6}$ Depending on the organs involved and the severity of granulomatous inflammation, patients suffer from a broad range of symptoms. In addition to organ-related symptoms, patients often suffer from disabling non-specific, non-organ related symptoms. ${ }^{7-9}$ Fatigue is the most frequently reported non-specific burdensome symptom in sarcoidosis patients, with a prevalence ranging from 50 to $90 \%{ }^{10,11}$ Other non-specific symptoms accounting for an important impact on the quality of life (QoL) of both patients and partners are small fiber neuropathy (SFN) related symptoms and everyday cognitive failure, including concentration problems, memory loss and decreased perception. ${ }^{7,12-15}$ Moreover, clinicians tend to have more attention for physical parameters than psychological issues in patients with sarcoidosis. To date, drugs used to treat severe organ involvement in sarcoidosis generally do not influence these non-specific symptoms and tend to cause side effects which then further increase the burden of disease.

The importance of patients' participation in healthcare has been increasingly acknowledged. Moreover, studies from a patients' perspective are important. Patients reported they regularly feel misunderstood and would like more attention and support for their problems. ${ }^{13}$ Therefore, the aim of this study was to assess the self-reported burden of patients with sarcoidosis in three European countries, viz. Denmark, Germany and the Netherlands, especially regarding the prevalence of fatigue and SFNrelated symptoms, as well as differences in treatment strategies.

\section{Materials and methods}

\section{Study design}

In cooperation with the Dutch Sarcoidosis Society, the ild care foundation has designed a questionnaire to assess complaints (organ-related as well as non-specific, non-organ related) among patients with sarcoidosis. This cross-sectional web-based anonymous 
survey was conducted from October 2017 to April 2018 among a sample of sarcoidosis patients in the Netherlands, and from December 2017 to August 2018 among samples of sarcoidosis patients in Denmark and Germany. The recruitment procedure aimed to compose representative samples of sarcoidosis patients in these countries.

This study was performed in accordance with the Declaration of Helsinki and its amendments. The Medical Ethics Committee of the St. Antonius Hospital Nieuwegein, The Netherlands, decided that, under the Dutch act on medical research involving human subjects, approval of this study by a Medical Ethics Committee was not necessary.

\section{Study subjects and procedure}

The overall study sample comprised sarcoidosis patients who were members of the Dutch Sarcoidosis Society (total number of patient members about 2000) and the Deutsche Sarkoidose Vereinigung (total number of patients' members about 4000), and mainly from a sarcoidosis clinic in Denmark. Patients were recruited without incentives, since the survey was anonymous.

The survey was developed using the online questionnaire tool Surveymonkey (www.surveymonkey.com). The survey concerns the burden of disease and symptoms as experienced by patients with sarcoidosis. Further questions concerned demographics (gender, age, duration of sarcoidosis), use of medication and two sets of questionnaires validated for sarcoidosis, the Small Fiber Neuropathy Screening List $\left(\right.$ SFNSL) ${ }^{16}$ and the Fatigue Assessment Scale (FAS). ${ }^{17}$ Patients were provided with a link to the electronic survey.

\section{Questionnaires}

The FAS is a 10-item self-report fatigue questionnaire (see Appendix). The response scale is a five-point scale ( 1 never to 5 always); scores on the FAS can range from 10 to 50. A score $>22$ indicates fatigue and a score $>34$ indicates extreme fatigue. The reliability and validity of the FAS have been shown to be good in sarcoidosis patients. ${ }^{17}$ So far, the FAS is available in 20 languages $^{18}$ (http://www.wasog.org/educationresearch/questionnaires.html).

The SFNSL is a 21-item self-administered questionnaire to screen for symptoms related to SFN (see Appendix). The response scale is a five-point scale ( 0 never to 4 always); scores on the SFNSL can range from 0 to 84. The cut-off score of the SFNSL is 11: a score below 11 indicates no or few symptoms related to SFN, while a score of 11-48 indicates probable or highly probable SFN and a score above 48 is indicative of SFN. ${ }^{16}$ The SFNSL 
is available in six languages; Danish, Dutch, English, French, German, Italian and Japanese (http://www.wasog.org/education-research/questionnaires.html).

\section{Statistical analysis}

All statistical analyses were performed using SPSS version 24 for Mac. Standard descriptive statistics were computed. ANOVA was used for comparison between the sarcoidosis samples in the three countries. In view of the large number of variables examined, a probability value of less than 0.01 was considered to be statistically significant.

\section{Results}

The characteristics of the samples studied are summarized in Table 8.1. A total of 1072 sarcoidosis patients (152 [68\% female] Danish; 532 [62\% female] German and 388 [53\% female] Dutch) completed the survey. The mean age was 51.8 years and did not differ between the three countries.

Self-reported pulmonary involvement was the most common complaint (72.4\%), followed by musculoskeletal involvement (70.2\%). Rates of musculoskeletal, cardiac and liver sarcoidosis differed between the three European countries.

Almost all patients (95\%) reported having symptoms (organ-related as well as nonspecific, non-organ related). Of the non-specific, non-organ related symptoms, fatigue was reported by almost all sarcoidosis patients in all three countries (approximately $90 \%)$. SFN-related symptoms (86.2\%), reduced energy levels (80.6\%) and concentration (54.0\%), memory (51\%) and sleeping (50.5\%) problems were also often reported. None of these symptoms varied between the three European countries. Only memory problems tended to be more prevalent in Denmark $(58.1 \%, p=0.05)$. Significant differences were found regarding self-reported pain, which was least prevalent in the Netherlands $(p \leq 0.001)$, and low vitamin D levels, with the highest prevalence in Germany (31.7\%, $p=0.002)$.

More than $50 \%$ of the studied sample were being treated with prednisone, a rate which was comparable in all three countries. However, substantial differences were found concerning second- and third-line treatment. Methotrexate (MTX) was used more commonly in Denmark and the Netherlands than in Germany (23.5\% [DN] and $21.7 \%$ $[\mathrm{NL}$ ] versus $8.5 \% ; \mathrm{p} \leq 0.001)$, while azathioprine (AZA) was prescribed more often in Germany (9.2\% versus $7.4 \%[D N]$ and $3.8 \%[N L] ; p \leq 0.001)$. 
Table 8.1 Summary of the characteristics of the sarcoidosis patient samples from Denmark, Germany and the Netherlands.

\begin{tabular}{|c|c|c|c|c|}
\hline & Denmark & Germany & The Netherlands & $p$-value\# \\
\hline number & 152 & 532 & 388 & \\
\hline gender, male, \% & 31.9 & 38.0 & 46.6 & $<0.001$ \\
\hline age, years, mean $\pm S D$ & $52.1 \pm 9.9$ & $52.3 \pm 9.2$ & $51.1 \pm 9.7$ & NS \\
\hline (range) & $(12-72)$ & $(23-80)$ & $(24-75)$ & \\
\hline \multicolumn{5}{|l|}{ organ involvement, \% } \\
\hline pulmonary & 75.0 & 73.0 & 69.1 & NS \\
\hline cardiac & 2.1 & 7.8 & 5.4 & $<0.001$ \\
\hline musculoskeletal & 80.9 & 69.3 & 60.5 & $<0.001$ \\
\hline skin & 31.6 & 35.8 & 27.3 & NS \\
\hline low vitamin D & 27.9 & 31.7 & 20.4 & 0.002 \\
\hline hypercalcemia & 9.6 & 4.5 & 6.7 & NS \\
\hline nervous system & 10.3 & 11.9 & 11.6 & NS \\
\hline liver & 4.4 & 14.8 & 6.7 & $<0.001$ \\
\hline \multicolumn{5}{|l|}{ symptoms, \% } \\
\hline none & 4.4 & 4.7 & 1.5 & NS \\
\hline organ-related symptoms, \% & 95.6 & 95.3 & 95.9 & NS \\
\hline \multicolumn{5}{|l|}{ pulmonary } \\
\hline dyspnoea & 65.4 & 62.3 & 61.1 & NS \\
\hline cough & 49.3 & 42.1 & 38.1 & 0.05 \\
\hline extrapulmonary & 95.6 & 94.6 & 90.7 & NS \\
\hline cardiac arrhythmia* & 14.7 & 18.7 & 28.4 & $<0.001$ \\
\hline dizziness/fainting & 33.8 & 29.4 & 29.3 & $<0.001$ \\
\hline kidney stones & 5.1 & 5.1 & 6.2 & NS \\
\hline non-organ-related symptoms, \% & 95.6 & 97.7 & 94.4 & NS \\
\hline fatigue & 89.9 & 89.7 & 90.7 & NS \\
\hline pain & 74.5 & 68.9 & 62.5 & $<0.001$ \\
\hline reduced energy levels & 80.9 & 82.1 & 78.7 & NS \\
\hline concentration problems & 51.5 & 54.2 & 56.2 & NS \\
\hline memory problems & 58.1 & 46.9 & 47.9 & 0.05 \\
\hline sleeping problems & 56.6 & 50.4 & 44.6 & NS \\
\hline restless legs & 36.8 & 32.1 & 33.2 & NS \\
\hline dry or running eyes & 43.4 & 42.6 & 39.7 & NS \\
\hline \multicolumn{5}{|l|}{ medication, \% } \\
\hline none, ever & 27.1 & 32.0 & 33.2 & NS \\
\hline predniso(lo)n & 56.6 & 51.4 & 54.8 & NS \\
\hline methotrexate & 23.5 & 8.5 & 21.7 & $<0.001$ \\
\hline azathioprine & 7.4 & 9.2 & 3.8 & 0.01 \\
\hline hydroxychloroquine & 5.9 & 0.1 & 4.9 & $<0.001$ \\
\hline anti-TNF- $\alpha$ & 4.0 & 2.6 & 7.2 & $<0.001$ \\
\hline vitamin D & 39.1 & 40.5 & 29.8 & $<0.001$ \\
\hline calcium & 38.0 & 10.5 & 10.0 & $\begin{array}{l}\text { NS (DN vs GE and } \\
\text { DN vs NL <0.001) }\end{array}$ \\
\hline pain killers or NSAIDs & 35.3 & 48.6 & 32.7 & 0.005 \\
\hline sleeping medication & 5.9 & 4.3 & 8.5 & NS \\
\hline
\end{tabular}

TNF- $\alpha=$ tumour necrosis factor alpha; NS=non-significant; NSAID=non-steroidal anti-inflammatory drug; $\mathrm{DN}=$ Denmark; GE=Germany; NL=the Netherlands; *patient's experience not confirmed by a cardiologist; \#=significantly different between all 3 countries (DE, GE and NL). 
Tumour-necrosis-factor alpha (TNF- $\alpha$ ) inhibitors were used most frequently in the Netherlands (7.2\% versus $4.0 \%[\mathrm{DN}]$ and $2.6 \%$ [GE]; $\mathrm{p} \leq 0.001)$. In total, $34.9 \%$ of the patients in Denmark, $32.7 \%$ of the patients in the Netherlands and $20.3 \%$ of the patients in Germany were receiving second- or third-line treatment. The use of calcium supplementation was higher in Denmark (38.0\% [DN] versus $10.5 \%$ [GE] and $10.0 \%$ $[N L] ; p \leq 0.001$ ), and more German patients reported using pain killers (including opioids).

Based on the assumption that an FAS score above 22 indicates fatigue, approximately $90 \%$ of the sarcoidosis patients in all three countries were affected by fatigue. The mean FAS score in the three European countries was 32.1 (the highest score being found in the Netherlands: $33.1 ; p \leq 0.001$; see Table 8.2). Patients in the Netherlands and Denmark reported more extreme fatigue (FAS >34) than those in Germany (47.9\% [NL] and $46.3 \%$ [DN] versus 33.7\% [GE]; $p \leq 0.001$ (see also Figure $8.1 \mathrm{a}$ ).

Table 8.2 Fatigue Assessment Scale (FAS) and Small Fiber Neuropathy Screening List (SFNSL) scores of the sarcoidosis samples from Denmark, Germany and the Netherlands.

\begin{tabular}{lllcc}
\hline & Denmark & Germany & The Netherlands & $p$-value \\
\hline FAS, total \pm SD & $32.3 \pm 8.4$ & $30.8 \pm 7.7$ & $33.1 \pm 8.1$ & $<0.001$ \\
FAS, mental \pm SD & $14.6 \pm 4.6$ & $13.8 \pm 4.2$ & $15.0 \pm 4.4$ & $<0.001$ \\
FAS, physical \pm SD & $17.7 \pm 4.5$ & $17.4 \pm 9.1$ & $18.1 \pm 4.2$ & NS \\
SFNSL, total $\pm S D$ & $32.4 \pm 16.4$ & $26.6 \pm 17.8$ & $27.9 \pm 18.7$ & 0.004 \\
\hline
\end{tabular}

$\mathrm{SD}=$ standard deviation ; NS=non-significant

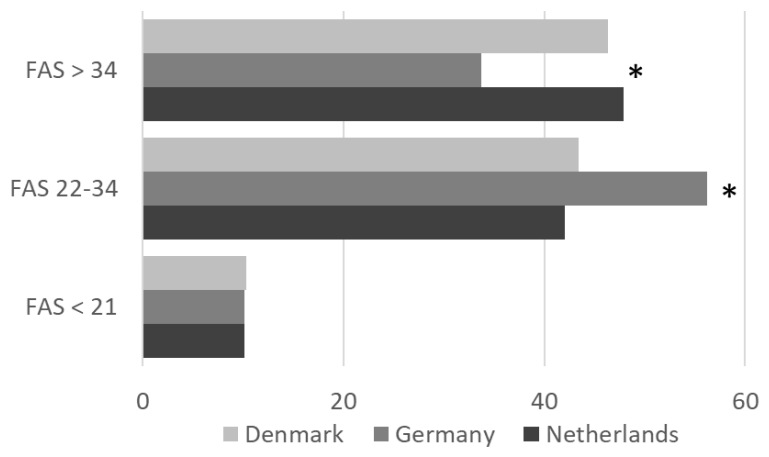

Figure 8.1a Fatigue Assessment Scale (FAS) subscores per country. FAS scores below 22 indicate no fatigue, scores between 22 and 34 indicate mild to moderate fatigue, and scores above 34 indicate severe fatigue. ${ }^{18} *=p$-value $<0.001$ (GE vs. DN+NL). 
Based on the assumption that an SFNSL score $>11$ indicates probable or highly probable SFN, $>80 \%$ of the sarcoidosis patients in all three countries are affected by SFN-related symptoms. The mean SFNSL score in the three European countries was 29 (the highest score being found in Denmark: 32.4; $p=0.004)$. The highest prevalence of SFN-related symptoms was seen in Denmark (91.9\%), followed by Germany $(84.6 \%)$. In the Netherlands, $82.2 \%$ of the patients reported SFN-related symptoms (see also Table 8.2). The prevalence of scores $>48$ (indicative of SFN) was also substantial: $18.5 \%$ in Denmark, $12.3 \%$ in Germany and $15.5 \%$ in the Netherlands (see also Figure $8.1 \mathrm{~b}$ ).

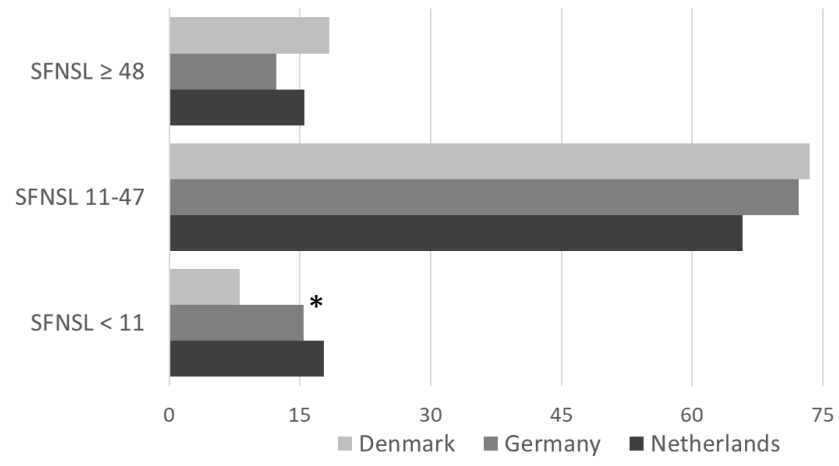

Figure 8.1b Small Fiber Neuropathy Screening List (SFNSL) subscores per country. A score below 11 indicates few or no SFN-related symptoms, a score between 11 and 48 indicates probable or highly likely SFN, and a score above 48 is indicative of SFN. ${ }^{16}=$ p-value $<0.001$ (DN vs. GE + NL)

\section{Discussion}

Our findings demonstrate that the self-reported burden of sarcoidosis is high. Almost all patients reported sarcoidosis-associated symptoms (organ-related as well as nonspecific, non-organ related). Of the non-specific symptoms, fatigue (assessed by the FAS) and reduced energy levels were reported by almost all sarcoidosis patients in the three European countries we studied (approximately $90 \%$ and $80 \%$, respectively). In addition, over $80 \%$ of the respondents reported SFN-related symptoms (as assessed by the SFNSL). Pulmonary involvement was the most frequently organ-related manifestation reported, followed by musculoskeletal manifestations.

Difficulties arise when estimating the real extent and prevalence of organ involvement and non-specific symptoms associated with sarcoidosis in a community. Descriptions differ widely among populations in the world and even between various regions within 
countries, as standardization of the diagnostic criteria is still lacking. ${ }^{1}$ To date, epidemiological data on the prevalence of various manifestations in the European countries we studied are sparse. In Denmark, 50\% of the cases in a National Patient Registry were apparently asymptomatic. ${ }^{1} \mathrm{New}$ imaging tests such as positron emission tomography (PET)-scans, revealing occult sarcoidosis localizations and/or multiple organ involvement, have led to changes in reported manifestations. ${ }^{5,19}$ In the present study, the reported proportion of organ involvement was comparable between the three countries, except for cardiac and liver involvement (both most prevalent in Germany).

Clinically apparent cardiac involvement has been noted in $2-10 \%$ of patients, ${ }^{20}$ while the prevalence of clinically silent cardiac sarcoidosis is much higher. Advanced imaging, including PET-scans and magnetic resonance imaging (MRI) have improved the detection of cardiac sarcoidosis. ${ }^{20}$ Apparently, there are differences in the presentation of sarcoidosis in this respect between patients from Europe and America and those from Japan, as Japanese studies have reported much higher rates of cardiac involvement. The prevalence of cardiac involvement reported in the present study was similar to previously reported rates (8\%) in large sarcoidosis samples in Germany. ${ }^{2,21}$ In contrast, the present study showed a rather lower prevalence in Denmark (2.1\%), which is not consistent with previously reported rates in Denmark (8.6\%). ${ }^{22}$ This difference may be due to the fact that our Danish study sample as well as the previous study were conducted in a single centre. ${ }^{22}$ Moreover, the prevalence of manifestations also depends on the available diagnostic procedures, the expertise of the clinicians and the definition used to determine organ involvement in various centres.

Liver problems in sarcoidosis could be associated with, at least partly, hepatic sarcoidosis, hepatotoxic medication such as azathioprine and/or alcohol intake. In line with the findings of the present study, Kirsten and Bosse-Henck et al. reported high rates of liver involvement (15\%) in large sarcoidosis samples in Germany. ${ }^{2,21}$ Recently, liver involvement was found in approximately $15 \%$ of a Dutch sarcoidosis sample, which is higher than reported in the present study (6.7\%). ${ }^{23}$ This can probably be explained by the fact that the sample studied by Cremers et al. included more chronic, advanced sarcoidosis patients, since their study was performed in a tertiary referral centre. So far, comparative epidemiologic data on liver involvement in Denmark are lacking.

Organ-specific symptoms were comparable between the three countries, except for musculoskeletal symptoms (being most prevalent in Denmark). Musculoskeletal manifestations occur in approximately one-third of sarcoidosis patients. ${ }^{24}$ Since they are often subclinical or not recognized because of mild or non-specific symptoms, however, their exact prevalence is unknown. The most frequent musculoskeletal manifestation of sarcoidosis is an acute arthritis that occurs as part of the Löfgren 
syndrome. ${ }^{24}$ Higher rates of musculoskeletal involvement have been reported since the introduction of the PET-CT. ${ }^{25}$ Awareness of organ involvement and sarcoidosisassociated symptoms will continue to increase with the improvement of diagnostic options and more prominent patient participation in the management of their disease.

In line with previous studies, the present study found that non-specific symptoms were frequently reported by sarcoidosis patients. ${ }^{9-11,13,14,26}$ Hinz et al. reported fatigue in $70 \%$ of the German sarcoidosis population (mean FAS score: 26.3 ) $^{26}$ and De Kleijn et al. reported fatigue in $83 \%$ of the Dutch sarcoidosis population (mean FAS score: 30.3 ). ${ }^{28}$ To date, ours was the first study evaluating fatigue in Denmark, so no previous data exist. These findings underline the clinical importance of assessing fatigue in sarcoidosis and integrating it in the multidisciplinary management of this disease. The aetiology of sarcoidosis-associated fatigue is poorly understood and is likely to be multifactorial, encompassing active inflammation, cytokine release, depressive symptoms, sleep disturbance, anxiety, everyday cognitive failure and/or SFN-related symptoms. ${ }^{7,11}$ Fatigue can also be caused by systemic treatments for sarcoidosis, such as corticosteroids. ${ }^{29,30}$ Recently, our group demonstrated that predictors of fatigue include everyday cognitive failure, SFN-associated symptoms, depressive symptoms, anxiety, muscle pain and dyspnea. ${ }^{8}$ Although our survey did not include a depressive symptoms inventory, it can be concluded from earlier studies by our group and others that the presence of depressive symptoms is one of the most important predictors of fatigue. ${ }^{8,21}$ Moreover, more than fifty percent of the patients in our sample reported memory and concentration problems, symptoms associated with cognitive failure and fatigue. ${ }^{8}$

Ours was also the first study to assess SFN-related symptoms among sarcoidosis patients in Denmark and Germany. SFN-related symptoms, including pain, were frequently reported by all participants in the present study. In the follow-up of sarcoidosis patients, routine tests to assess disease activity do not measure pain, so the results of tests do not always correlate with the patients' well-being. ${ }^{4}$ Since pain is a substantial problem in sarcoidosis, appropriate questionnaires to obtain information about pain might be helpful. ${ }^{9}$

The percentage of patients using drugs was comparable in the three European countries (around 70\%). However, treatment strategies differed between these three countries, a difference which was largely caused by the difference in second- and thirdline treatment (20\% [GE] versus 33\% [NL] and 35\% [DN]). Patients in Denmark and the Netherlands were more likely to use methotrexate and TNF- $\alpha$ inhibitors, whereas in Germany azathioprine was regarded as the treatment of first choice when prednisone alone was not effective. Remarkably, TNF- $\alpha$ inhibitors were prescribed most frequently in the Netherlands. This can most probably be explained by differences between the three countries in the health care insurance systems and/or local guidelines. 


\section{Limitations}

One of the limitations of the present study was the patient recruitment method used in the three countries. The majority of the patients were recruited through patient societies. One could argue that it is only symptomatic patients who become members of a patients' society or are specifically referred to a specialized sarcoidosis clinic, and so these were more likely to take part in the survey than asymptomatic patients, causing selection bias and considerably influencing the rate of symptomatic cases in this study. Therefore, these results are only applicable to symptomatic patients. At present the rate of symptoms in the unselected nationwide sarcoidosis population remains unknown. Another limitation is the fact that the symptoms were self-reported, which could lead to bias. However, the burden of sarcoidosis is determined by the experience of the patients themselves, and we barely saw any differences in prevalence rates of sarcoidosis-associated symptoms between the European countries we studied.

\section{Conclusion}

This study shows that sarcoidosis patients in Denmark (DN), Germany (GE) and the Netherlands (NL) report similar sarcoidosis-associated symptoms, including organrelated as well as non-specific, non-organ related symptoms. Fatigue (90\%) and symptoms associated with SFN (mean 86\%: DN 91.9\%, GE $84.6 \%$ and NL $82.2 \%$, respectively) were highly prevalent in all 3 countries.

Beside better disease education, psychological support is warranted. The combined use of appropriate questionnaires, lung function tests, imaging procedures and other clinical assessments of disease activity and severity provides a framework for evaluating organ-related as well as non-organ related symptoms.

In view of the broad range of possible symptoms, sarcoidosis patients may consult various doctors, so the management of sarcoidosis patients should use a multidisciplinary approach that focuses on somatic as well as psychosocial aspects of this erratic disorder. Furthermore, as treatment strategies differ in the three European countries we studied, updated international sarcoidosis treatment guidelines are urgently needed. 


\section{References}

1. Byg KE, Milman N, Hansen S. Sarcoidosis in Denmark 1980-1994. A registry-based incidence study comprising 5536 patients. Sarcoidosis Vasc Diffuse Lung Dis 2003;20(1):46-52.

2. Kirsten D. [Sarcoidosis in Germany. Analysis of a questionnaire survey in 1992 of patients of the German Sarcoidosis Group]. Pneumologie 1995;49(6):378-82.

3. Valeyre D, Prasse A, Nunes H, et al. Sarcoidosis. Lancet 2014;383(9923):1155-67.

4. Wirnsberger RM, de Vries J, Breteler $\mathrm{MH}$, et al. Evaluation of quality of life in sarcoidosis patients. Respir Med 1998;92(5):750-6.

5. Gerke AK, Judson MA, Cozier YC, et al. Disease burden and variability in sarcoidosis. Ann Am Thorac Soc 2017;14(Supplement_6):S421-8.

6. Statement on sarcoidosis. Joint Statement of the American Thoracic Society (ATS), the European Respiratory Society (ERS) and the World Association of Sarcoidosis and Other Granulomatous Disorders (WASOG) adopted by the ATS Board of Directors and by the ERS Executive Committee, February 1999. Am J Respir Crit Care Med 1999;160(2):736-55.

7. Drent M, Strookappe B, Hoitsma E, De Vries J. Consequences of sarcoidosis. Clin Chest Med 2015; 36(4):727-37.

8. Hendriks C, Drent M, De Kleijn W, et al. Everyday cognitive failure and depressive symptoms predict fatigue in sarcoidosis: a prospective follow-up study. Respir Med 2018;138S:S24-30.

9. Hoitsma E, De Vries J, van Santen-Hoeufft M, et al. Impact of pain in a Dutch sarcoidosis patient population. Sarcoidosis Vasc Diffuse Lung Dis 2003;20(1):33-9.

10. Marcellis RG, Lenssen AF, Elfferich $M D$, et al. Exercise capacity, muscle strength and fatigue in sarcoidosis. Eur Respir J 2011;38(3):628-34.

11. Drent M, Lower EE, De Vries J. Sarcoidosis-associated fatigue. Eur Respir J 2012;40(1):255-63.

12. Bakkers M, Faber CG, Hoeijmakers JG, et al. Small fibers, large impact: quality of life in small-fiber neuropathy. Muscle Nerve 2014;49(3):329-36.

13. Moor CC, van Manen MJG, van Hagen PM, et al. Needs, perceptions and education in sarcoidosis: a live interactive survey of patients and partners. Lung 2018;196(5):569-75.

14. Voortman M, Fritz D, Vogels OJM, et al. Small fiber neuropathy: a disabling and underrecognized syndrome. Curr Opin Pulm Med 2-17;23(5):447-57.

15. Elfferich MD, Nelemans PJ, Ponds RW, et al. Everyday cognitive failure in sarcoidosis: the prevalence and the effect of anti-TNF-alpha treatment. Respiration 2010;80(3):212-9.

16. Hoitsma E, De Vries J, Drent M. The small fiber neuropathy screening list: construction and crossvalidation in sarcoidosis. Respir Med 2011;105(1):95-100.

17. De Vries J, Michielsen $H$, Van Heck GL, Drent $M$. Measuring fatigue in sarcoidosis: the Fatigue Assessment Scale (FAS). Br J Health Psychol 2004;9(Pt 3):279-91.

18. Hendriks C, Drent M, Elfferich M, De Vries J. The Fatigue Assessment Scale: quality and availability in sarcoidosis and other diseases. Curr Opin Pulm Med 2-18;24(5):495-503.

19. Ganeshan D, Menias CO, Lubner MG, et al. Sarcoidosis from head to toe: what the radiologist needs to know. Radiographics 2018;38(4):1180-200.

20. Bakker AL, Grutters JC, Keijsers RG, Post MC. Cardiac sarcoidosis: challenges in clinical practice. Curr Opin Pulm Med 2017;23(5):468-75.

21. Bosse-Henck A, Koch $R$, Wirtz $H$, Hinz A. Fatigue and excessive daytime sleepiness in sarcoidosis: prevalence, predictors, and relationships between the two symptoms. Respiration 2017;94(2):186-97.

22. Ghanizada M, Rossing K, Bundgaard H, Gustafsson F. Clinical presentation, management and prognosis of patients with cardiac sarcoidosis. Dan Med J 2018;65(4).

23. Cremers J, Drent M, Driessen A, et al. Liver-test abnormalities in sarcoidosis. Eur J Gastroenterol Hepatol 2012;24(1):17-24.

24. Bechman K, Christidis D, Walsh S, et al. A review of the musculoskeletal manifestations of sarcoidosis. Rheumatology (Oxford) 2018;57(5):777-83.

25. Mostard RL, Prompers L, Weijers RE, et al. F-18 FDG PET/CT for detecting bone and bone marrow involvement in sarcoidosis patients. Clin Nucl Med 2012;37(1):21-5. 
26. Hinz A, Fleischer $M$, Brahler $E$, et al. Fatigue in patients with sarcoidosis, compared with the general population. Gen Hosp Psychiatry 2011;33(5):462-8.

27. Korenromp IHE, Heijnen CJ, Vogels OJM, et al. Characterization of chronic fatigue in patients with sarcoidosis in clinical remission. Chest 2011;140(2):441-7.

28. de Kleijn WP, Elfferich MD, De Vries J, et al. Fatigue in sarcoidosis: American versus Dutch patients. Sarcoidosis Vasc Diffuse Lung Dis 2009;26(2):92-7

29. Fleischer M, Hinz A, Brahler E, et al. Factors associated with fatigue in sarcoidosis. Respir Care 2014;59(7):1086-94.

30. Judson MA, Chaudhry $\mathrm{H}$, Louis $\mathrm{A}$, et al. The effect of corticosteroids on quality of life in a sarcoidosis clinic: the results of a propensity analysis. Respir Med 2015;109(4):526-31. 



\section{Chapter}

\section{Quality of life of couples living with sarcoidosis}

M. Voortman, C.M.R. Hendriks, P. Lodder, M. Drent, J. De Vries

Respiration; 2019 Epub 


\section{Abstract}

\section{Background}

Consequences of sarcoidosis are wide-ranging and the symptom burden has a great impact on patients' quality of life (QoL). However, the QoL of couples living with sarcoidosis has not yet been studied. Our aim was to assess the QoL of couples living with sarcoidosis and to evaluate whether living with a partner with sarcoidosis influences the partner's QoL. Furthermore, we aimed to assess whether non-specific symptoms (fatigue, cognitive failure, small fiber neuropathy (SFN) related symptoms, depressive symptoms, and state/trait anxiety) predict QoL of partners as well as sarcoidosis patients.

\section{Methods}

Sarcoidosis outpatients, recruited at Maastricht University Medical Centre $(n=443)$, and their partners $(n=208)$, completed several questionnaires, including the World Health Organization Quality of Life - BREF (WHOQoL-BREF), Fatigue Assessment Scale (FAS), SFN screening list (SFNSL), and Cognitive Failure Questionnaire (CFQ).

\section{Results}

QoL of the partners as well as the sarcoidosis patients was reduced compared with healthy controls, especially regarding the physical health domain. All non-specific symptoms studied, as well as perceived social support, predicted one or more QoL domains in the sarcoidosis patients, but these factors did not predict the QoL of their partners.

\section{Conclusions}

The QoL of partners of sarcoidosis patients was reduced, although to a lesser extent than that of the patients. Although the non-specific symptoms and perceived social support were related to the patients' QoL, this was not the case for the partners. In the management of sarcoidosis is it important to focus not only on the patients, but also on their partners. 


\section{Introduction}

Sarcoidosis is a multisystem inflammatory disorder of unknown cause(s) that imposes a burden on patients' lives. ${ }^{1}$ It affects men and women all over the world, at a relatively young age. ${ }^{2}$ In addition to the specific organ-related symptoms, less specific disabling symptoms, including fatigue, cognitive failure, symptoms associated with small fiber neuropathy (SFN), and physical impairments, may have a major influence on the daily activities and the social and professional lives of the patients. These symptoms can lead to stress, anxiety, depression, and social and physical limitations. ${ }^{3-8}$ Moreover, sarcoidosis patients experience reduced work ability. ${ }^{9-11}$

The impact of any disease depends on the patient's disease perception and coping strategies. Various aspects of sarcoidosis, such as the often unpredictable and chronic nature of the disease, ${ }^{2}$ the uncertainty about the cause, and the broad range of frequently persistent symptoms may result in an aggravating influence on patients' lives. Hence, living with a long-term disease like sarcoidosis significantly affects patients' quality of life ( $Q \mathrm{oL})$, with negative consequences for general health and social and psychosocial well-being. QoL is a concept that concerns someone's evaluation of their functioning in a wide range of domains, but always includes the physical, psychological, and social domains. Anxiety and depressive symptoms are also common in patients with sarcoidosis and are associated with disease severity. ${ }^{6,7}$ Previous QoL studies showed that the QoL of patients with sarcoidosis is predicted by fatigue, depressive symptoms, reduced exercise capacity, dyspnea, and arthralgia., ${ }^{1,4,8,12-16}$ Recently, Moor et al. demonstrated that sarcoidosis not only leads to anxiety, psychological distress and impaired well-being in the patients, but also in their partners. ${ }^{17}$ Studies on the influence of other non-specific symptoms and studies examining the impact of living with a partner with sarcoidosis are scarce.

Therefore, the aim of our study was to evaluate whether living with a partner suffering from sarcoidosis influences the QoL of that partner, as well as to evaluate the QoL of the patients themselves. Moreover, we aimed to assess whether non-specific symptoms, including fatigue, cognitive failure, SFN-related symptoms, and psychological factors including depressive symptoms and anxiety in sarcoidosis patients predict the QoL of partners and patients. 


\section{Materials and methods}

\section{Study design and subjects}

We conducted a cross-sectional, prospective cohort study evaluating fatigue and QoL in sarcoidosis patients and their partners between 2007 and 2009. Data regarding fatigue in sarcoidosis patients were previously used in Willemien de Kleijn's PhD project. ${ }^{18}$ Data from the current study regarding the QoL of sarcoidosis patients and their partners were analyzed in 2018, and not reported previously. Patients $(n=588)$ evaluated and/or treated at the ILD Center of the Department of Pulmonology of Maastricht University Medical Center, a tertiary referral center in the Netherlands were asked to participate between September 2007 and July 2008. After inclusion of the patients, their partners (if they had a partner) were also asked to participate. Patients were all diagnosed with sarcoidosis based on the guidelines of the World Association of Sarcoidosis and Other Granulomatous Disorders (WASOG). ${ }^{2}$ The Medical Ethics Committee of MUMC+ (MEC 07-4-015) approved the study protocol, and written informed consent was obtained from all patients.

\section{Procedure}

Information about the study was sent by e-mail (which every patient and/or partner asked to participate had access to), and patients as well as their partners were asked to complete and return an informed consent form if they were willing to participate. After having agreed to participate, they received the set of questionnaires and were asked to return the completed set to the hospital in an enclosed envelope.

The following characteristics were collected: gender and age (patients and partners), and time since diagnosis of sarcoidosis and sarcoidosis treatment (patients). Patients completed several questionnaires: World Health Organization Quality of Life-BREF (WHOQoL-Bref), Fatigue Assessment Scale (FAS), Small Fiber Neuropathy Screening List (SFNSL), Cognitive Failure Questionnaire (CFQ), State and Trait Anxiety Inventory (STAI), Center for Epidemiological Studies Depression Scale (CES-D) and Perceived Social Support Scale (PSSS). The partners were asked to complete the WHOQoL-BREF, FAS, and CFQ.

\section{Questionnaires}

Questionnaire scores of patients and partners in the present study were compared with corresponding questionnaire scores of a healthy control sample $(n=62)$ recruited for a 
study by Marcellis et al.. ${ }^{19}$ These healthy controls were recruited in the same geographical area/institution and timeframe as our study sample. We used the raw data of this healthy control population.

Detailed descriptions of the questionnaires (WHOQoL-BREF, FAS, SFNSL, CFQ, STAI, CES$D$, PSSS) can be found in the online supplement. ${ }^{20-26}$

\section{Statistical analysis}

All statistical analyses were performed using SPSS version 24 for Mac. The chi-square test, the independent samples t-test (partners vs controls and patients vs controls) and paired t-tests (patients vs partners) were used to test for statistically significant differences between patients, their partners and controls. Hierarchical linear regression analyses were used to assess the associations between the scores for the various domains of the sarcoidosis patients' and partners' WHOQoL-BREF and the sarcoidosis patients' fatigue (FAS), SFN-associated symptoms (SFNSL), cognitive failure (CFQ), depression (CESD), anxiety (STAI) and perceived social support (PSSS). Adjustments were made for age, sex, treatment and time since diagnosis in the analysis regarding patients, and for age and sex in the analysis regarding partners. Since each WHOQoLBREF domain was analyzed by means of a separate regression model, a Bonferroni correction was used to reduce the risk of false positive findings, by adjusting the significance level in these 5 regression analyses from 0.05 to 0.01 . Hence, within each of the ten WHOQoL-BREF regression models (five for patients and five for partners) $p$-values of less than 0.01 were considered to indicate statistical significance. In view of this correction, we also reported $99 \%$ confidence intervals (instead of the conventional 95\% confidence intervals) around the estimated regression coefficients.

In a posthoc analysis we have performed a mediation analysis using the Baron \& Kenny method. ${ }^{27}$ A moderation analysis was performed by including the product of two meancentered predictors in a regression analysis, on top of the main effects of these two predictors.

\section{Results}

Three quarters of the approached sarcoidosis patient sample (443/588) agreed to participate, of whom 10 were excluded from further analyses because they did not complete the main outcome measure QoL. Two-hundred and eight partners decided to participate and completed the questionnaires. The sarcoidosis patients whose partners 
participated in the study $(n=208)$ did not differ from those without a partner or whose partners did not participate ( $n=225)$, except for age (see Table 9.1).

Table 9.1 summarizes the demographic and clinical data and the questionnaire scores of the 433 included patients are summarized. The prevalence of the various nonspecific symptoms among the sarcoidosis patients and their partners is shown in Figure 9.1.

Table 9.1 Summary of the demographic and clinical characteristics of the sarcoidosis patient sample studied.

\begin{tabular}{lccc}
\hline & $\begin{array}{c}\text { Total sarcoidosis } \\
\text { sample }(\mathrm{n}=433)\end{array}$ & $\begin{array}{c}\text { Sarcoidosis patients } \\
\text { included in partner } \\
\text { analysis }(\mathrm{n}=208)\end{array}$ & $\begin{array}{c}\text { Sarcoidosis patients } \\
\text { excluded from partner } \\
\text { analysis }(\mathrm{n}=225)\end{array}$ \\
\hline male: $\mathrm{n}(\%)$ & $233(53.8)$ & $108(51.9)$ & $126(55.5)$ \\
age, years (range) & $48 \pm 11.1(19-79)$ & $46.5 \pm 10.2(27-79)$ & $49.3 \pm 11.7(19-78)^{*}$ \\
time since diagnosis, years (range) & $7.7 \pm 7.8(0-65)$ & $7.2 \pm 6.4(0-44)$ & $8.1 \pm 8.9(0-65)$ \\
medication & & & \\
none (\%) & $157(36.3)$ & $71(34.1)$ & $86(37.9)$ \\
prednisone (\%) & $219(50.6)$ & $105(50.5)$ & $116(51.1)$ \\
methotrexate (\%) & $74(17.1)$ & $38(18.3)$ & $36(15.9)$ \\
TNF- $\alpha$ antagonists (\%) & $38(8.8)$ & $21(10.1)$ & $17(7.5)$ \\
FAS score & $29.3 \pm 8.4$ & $30.1 \pm 8.5$ & $28.7 \pm 8.3$ \\
SFNSL score & $29.9 \pm 15.6$ & $24.8 \pm 15.8$ & $23.1 \pm 15.4$ \\
CFQ score & $36.6 \pm 16.1$ & $43 \pm 17.1$ & $34.8 \pm 16.1$ \\
STAI & & & $11.6 \pm 3.8$ \\
state & $11.6 \pm 3.7$ & $11.5 \pm 3.5$ & $40.6 \pm 7.7$ \\
trait & $40.6 \pm 7.7$ & $40.6 \pm 7.7$ & $14.5 \pm 9.9$ \\
CESD & $14.2 \pm 9.7$ & $14 \pm 9.5$ & $61.8 \pm 13.4$ \\
PSSS & $62.5 \pm 13.4$ & $63.3 \pm 13.4$ & $12.3 \pm 3.1$ \\
WHOQoL-BREF & & & $14.1 \pm 2.4$ \\
1. physical health & $12.2 \pm 3.1$ & $12.2 \pm 3$ & $14.0 \pm 2.9$ \\
2. psychological health & $14.2 \pm 2.3$ & $14.3 \pm 2.3$ & $15.2 \pm 2.6$ \\
3. social relationships & $14.2 \pm 2.8$ & $14.4 \pm 2.8$ & $6 \pm 1.6$ \\
4. environment & $15.5 \pm 2.5$ & $15.8 \pm 2.4$ & $6 \pm 1.6$ \\
5. overall QoL & $6 \pm 1.6$ & & \\
\hline
\end{tabular}

Data are expressed as mean $\pm S D$ or range in parentheses if appropriate. CESD=Center for Epidemiological Studies-Depression Scale; CFQ=Cognitive Failure Questionnaire; FAS=Fatigue Assessment Scale; PSSS=Perceived Social Support Scale; SFNSL=Small Fiber Neuropathy Screening List; STAI=State and Trait Anxiety Inventory; TNF=tumour necrosis factor; WHOQoL-BREF=World Health Organization Quality of LifeBREF. * statistically significant: $p=0.01$. 


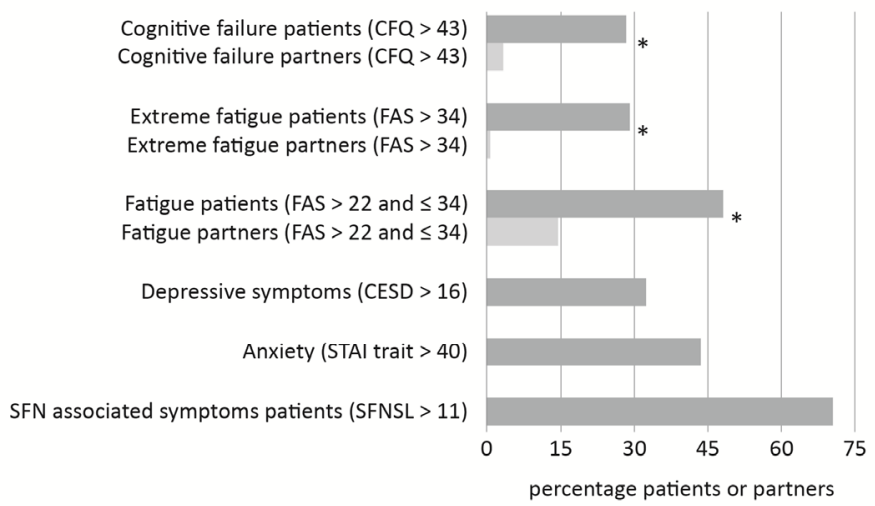

Figure 9.1 Non-specific symptoms in the sarcoidosis patients and their partners.

CESD=Center for Epidemiological Studies-Depression Scale; CFQ=Cognitive Failure Questionnaire; FAS=Fatigue Assessment Scale; SFNSL=Small Fiber Neuropathy Screening List; STAI=State and Trait Anxiety Inventory; ${ }^{*}=p<0.001$

\section{Quality of life partners and patients}

Table 9.2 summarizes the characteristics and outcomes of the partners of the sarcoidosis patients and the sarcoidosis patients themselves, and the comparison with healthy control subjects.

The overall QoL scores of the partners were lower than those of the healthy controls $(p<0.001)$. The QoL domain scores (Physical, Psychological, Social, Environment) were also lower for partners than for healthy controls.

The QoL of the sarcoidosis patients was significantly more impaired than that of their partners, overall as well as in the different domains (all p-values $<0.001$, except social relationships $\mathrm{p}=0.002$ ). 
Table 9.2 Characteristics of partners of sarcoidosis patients compared to those of sarcoidosis patients $(n=208)$ and healthy controls.

\begin{tabular}{lccccccc}
\hline & Partners & Patients & $\begin{array}{c}\text { Healthy } \\
\text { Controls }\end{array}$ & $\begin{array}{c}\Delta \text { Partners - Partners vs } \\
\text { patients }\end{array}$ & $\begin{array}{c}\text { Partners vs } \\
\text { Controls* }\end{array}$ & $\begin{array}{c}\text { Patients vs } \\
\text { Patients* }\end{array}$ \\
Controls*
\end{tabular}

Data are expressed as mean \pm SD if appropriate. CFQ=Cognitive Failure Questionnaire; FAS =Fatigue Assessment Scale; WHOQoL-BREF= World Health Organization Quality of Life-BREF. ${ }^{*}$ p-values resulting from a paired t-test testing for differences between the two groups.

\section{Associations of QoL of partners and sarcoidosis patients}

Hierarchical multiple linear regression analyses demonstrated that, after adjustment for the covariates age and gender of the partner, none of the non-specific symptoms (fatigue, SFN-associated symptoms, cognitive failure, state/trait anxiety, depressive symptoms and perceived social support) of the patients, including their QoL, were associated with the overall QoL score and those for the four QoL domains of their partners.

Hierarchical multiple linear regression analyses demonstrated that, after adjustment for the covariates age, gender, time since diagnosis and treatment, all non-specific symptoms we studied, except momentary anxiety, were associated with the overall QoL score and those for the four QoL domains of the sarcoidosis patients, predicting $32 \%$ to $63 \%$ of the variance (Table 9.3 ).

Depressive symptoms were negatively associated with the overall QoL score and those for all domains, except social relationships. Perceived social support was positively associated with all QoL domains, except physical health. Fatigue was negatively associated with the overall QoL score and those for the physical and psychological QoL domains. SFN-associated symptoms were negatively associated with the physical and 
environmental QoL domains. Trait anxiety was negatively associated with the psychological and social QoL domains. Finally, cognitive problems were only negatively associated with the QoL domain of physical health, but this association was subject to a mediation effect (see below).

To simplify, overall QoL is predicted by fatigue and depressive symptoms. The QoL domain physical health is predicted by fatigue, SFN-associated symptoms, depressive symptoms and cognitive failure. The QoL domain psychological health is predicted by fatigue, trait anxiety, depressive symptoms and perceived social support. The QoL domain social relationships is predicted by trait anxiety and perceived social support. And last, the QoL domain environment is predicted by SFN-associated symptoms, depressive symptoms and perceived social support (see Figure 9.2).

Table 9.3 Predictors of the WHOQoL-BREF.

\begin{tabular}{|c|c|c|c|c|c|}
\hline & \multicolumn{5}{|c|}{ WHOQoL-BREF } \\
\hline & Overall QoL score & 1. Physical health & $\begin{array}{l}\text { 2. Psychological } \\
\text { health }\end{array}$ & $\begin{array}{l}\text { 3. Social } \\
\text { relationships }\end{array}$ & 4. Environment \\
\hline \multirow[t]{2}{*}{$\overline{F A S}$} & $-0.10 *(-0.13,-0.08)$ & $-0.21 *(-0.26,-0.17)$ & $-0.06 *(-0.09,-0.02)$ & $-0.03(-0.08,0.03)$ & $-0.03(-0.10,0.05)$ \\
\hline & $\beta=-0.55$ & $\beta=-0.59$ & $\beta=-0.20$ & & \\
\hline \multirow[t]{2}{*}{ SFNSL } & $-0.01(-0.02,0.01)$ & $-0.04 *(-0.06,-0.02)$ & $0.02(0.00,0.03)$ & $-0.01(-0.04,0.02)$ & $-0.03 *(-0.06,-0.01)$ \\
\hline & & $\beta=-0.20$ & & & $\beta=-0.18$ \\
\hline \multirow[t]{2}{*}{ CFQ } & $0.01(0.00,0.03)$ & $0.02 *(0.00,0.05)$ & $0.00(-0.02,0.01)$ & $0.02(-0.01,0.05)$ & $0.01(-0.02,0.03)$ \\
\hline & & $\beta=0.12$ & & & \\
\hline \multirow[t]{2}{*}{ STAI Trait } & $0.00(-0.04,0.04)$ & $0.02(-0.05,0.08)$ & $-0.10 *(-0.15,-0.05)$ & $-0.10 *(-0.17,-0.02)$ & $-0.03(-0.10,0.05)$ \\
\hline & & & $\beta=-0.32$ & $\beta=-0.26$ & \\
\hline STAI State & $-0.03(-0.10,0.04)$ & $-0.11(-0.22,0.01)$ & $-0.06(-0.15,0.03)$ & $0.02(-0.12,0.16)$ & $-0.02(-0,16,0.12)$ \\
\hline \multirow[t]{2}{*}{ CESD } & $-0.04 *(-0.07,-0.01)$ & $-0.06^{*}(-0.11,-0.01)$ & $-0.08 *(-0.12,-0.03)$ & $-0.06(-0.13,0.00)$ & $-0.07 *(-0.13,-0.01)$ \\
\hline & $\beta=-0.24$ & $\beta=-0.17$ & $\beta=-0.31$ & & $\beta=-0.27$ \\
\hline \multirow[t]{2}{*}{ PSSS } & $0.00(-0.01,0.01)$ & $0.00(-0.02,0.02)$ & $0.02 *(0.01,0.04)$ & $0.07 *(0.05,0.10)$ & $0.03 *(0.01,0.06)$ \\
\hline & & & $\beta=0.11$ & $\beta=0.34$ & $\beta=0.17$ \\
\hline$R^{2}$ & $51 \%$ & $63 \%$ & $62 \%$ & $42 \%$ & $32 \%$ \\
\hline
\end{tabular}

CESD=Center for Epidemiological Studies-Depression Scale; CFQ=Cognitive Failure Questionnaire; FAS=Fatigue Assessment Scale; PSSS=Perceived Social Support Scale; SFNSL=Small Fiber Neuropathy Screening List; STAI=State and Trait Anxiety Inventory, WHOQoL-BREF=World Health Organization Quality of Life-BREF. Unstandardized regression coefficients and their $99 \%$ confidence intervals are shown. For significant effects, standardized regression coefficients are reported in bold face. $R^{2}=$ Percentage of additionally explained variance of the model including predictors above and beyond the model with theoretically important covariates only. ${ }^{*} p<0.01$. 


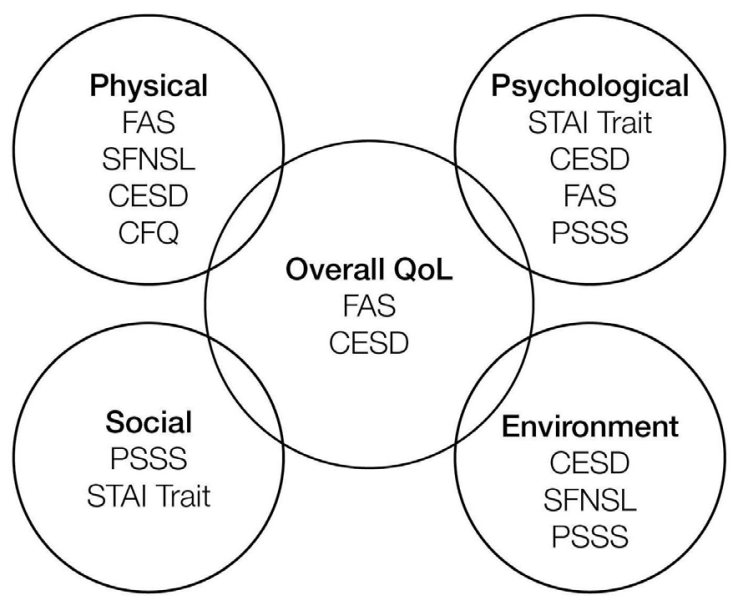

Figure 9.2 Simplification of the effect of predictors on overall quality of life (QoL) and the four domains as presented in Table 9.3.

CESD=Center for Epidemiological Studies-Depression Scale; CFQ=Cognitive Failure Questionnaire; FAS=Fatigue Assessment Scale; PSSS=Perceived Social Support Scale; SFNSL=Small Fiber Neuropathy Screening List; STAI=State and Trait Anxiety Inventory, WHOQoL-BREF=World Health Organization Quality of Life-BREF.

\section{Relative importance of the QoL predictors in partners and sarcoidosis patients}

The QoL predictors for partners were not significant, and are therefore not described any further here.

As regards the patients, the predictors explained $62.9 \%$ of the variance in the physical health domain scores. This domain was best predicted by fatigue, followed by SFNassociated symptoms, depressive symptoms, and cognitive failure.

The predictors explained $61.9 \%$ of the variance in the psychological health domain. This domain was best predicted by trait anxiety, depressive symptoms, fatigue, and perceived social support. Perceived social support had a positive effect on the psychological QoL, while the other variables had negative effects.

The predictors explained $41.7 \%$ of the variance in the social relationships domain. This domain was best predicted by trait anxiety and perceived social support. Perceived social support had a positive effect on QoL while trait anxiety had a negative effect. 
The predictors explained $31.8 \%$ of the variance in the environment domain. This domain was best predicted by the depressive symptoms, SFN-associated symptoms, and perceived social support. Again, perceived social support had a positive effect on QoL, while depressive symptoms and SFN-associated symptoms had a negative effect. The predictors explained $50.7 \%$ of the variance in the overall QoL score, and this was best predicted by fatigue and depressive symptoms.

\section{Mediation analysis}

The results in Table 9.3 suggest that cognitive failure had a positive effect on QoL (unstandardized B 0.024), indicating that patients with more cognitive failure had a higher physical QoL. Since cognitive failure has a great impact on the lives of sarcoidosis patients, and given that the present sample included mostly young patients with a busy social and working life, this finding was against our expectations. In regression models, a significant association between a predictor and outcome may in fact be explained by a third variable, such as a confounder, mediator or moderator. Therefore, we inspected the partial and zero-order correlations between the physical domain of QoL and the predictors. The zero-order correlations showed that there was, as expected, a negative association between CFQ and physical QoL. However, after adjusting for the influence of other predictors (primarily fatigue and depressive symptoms), this negative association disappeared through collinearity. Figure 9.3 shows two mediation models where fatigue and depressive symptoms, respectively, mediate the association between cognitive failure and physical QoL. Indeed, the significant zero-order correlation between these two measures disappeared when the mediating effects of fatigue and depressive symptoms were included in the model (see Figures 9.3A and 9.3B). Furthermore, a moderation analysis showed a significant interaction effect between the CFQ and FAS as well as CESD on physical QoL (CFQ-FAS: $\beta=0.078$, $\mathrm{SE}=0.001, \mathrm{t}=2.182, \mathrm{p}=0.03$ respectively CFQ-CESD: $\beta=0.176, \mathrm{SE}=0.001, \mathrm{t}=3.795$, $\mathrm{p}<0.001)$. These results suggest that there may be multiple mechanisms underlying the relations between these variables. 


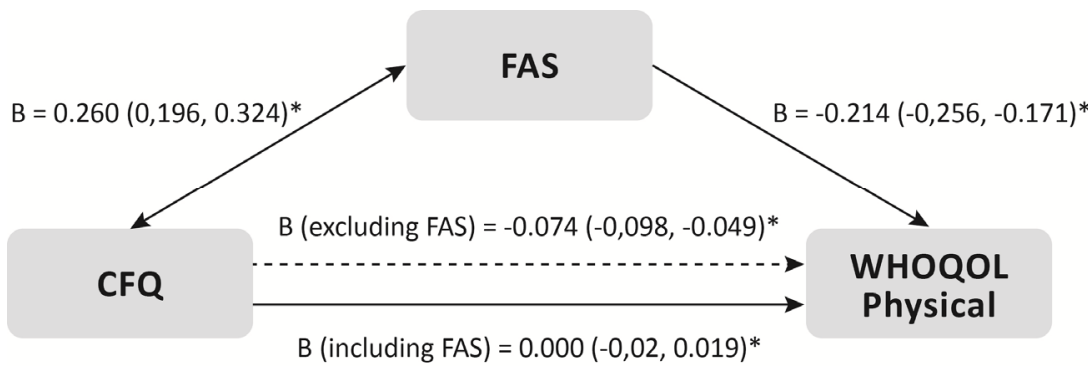

Figure 9.2A Impact of the Fatigue Assessment Scale (FAS) score on the Cognitive Failure Questionnaire (CFQ) regression coefficient in predicting the quality of Life (WHOQoL=World Health Organization Quality of Life). B=standardized regression coefficient, * = statistically significant

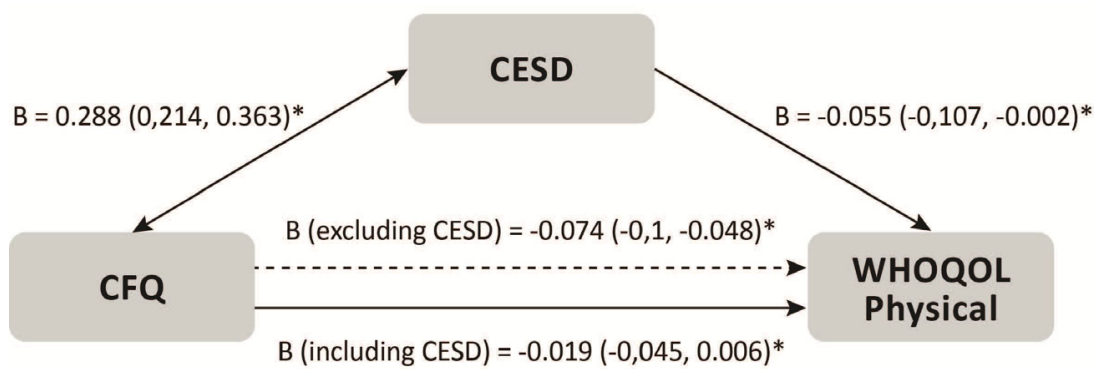

Figure 9.2B Impact of depressive symptoms score (CES-D=Center for Epidemiological Studies-Depression Scale) on Cognitive Failure Questionnaire (CFQ) regression coefficient in predicting the quality of life (WHOQoL=World Health Organization Quality of Life). B=standardized regression coefficient, ${ }^{*}=$ statistically significant.

\section{Discussion}

This is the first study to show that the QoL of couples where one of the partners suffers from sarcoidosis is reduced compared to that of healthy controls. Neither the QoL nor non-specific symptoms of the sarcoidosis patients predicted the QoL of the partners of the sarcoidosis patients we studied. Predictors of the QoL of the sarcoidosis patients themselves were fatigue, depressive symptoms, SFN-associated symptoms, cognitive failure, anxiety, and perceived social support. All of these non-specific symptoms, except perceived social support, had a negative impact on various aspects of the QoL of the sarcoidosis patients. This underlines the importance of determining all these nonspecific symptoms in sarcoidosis patients. 
The importance of patient-centeredness in healthcare has grown over the years. So far, family involvement and perspectives have infrequently been studied in sarcoidosis. A novel finding from our study is that partners of sarcoidosis patients also had a lower QoL than healthy persons, but to a lesser extent than that of the sarcoidosis patients we studied. The QoL of the partners living with a sarcoidosis patient was mainly lower in the psychological and social domains. We did not study the perceived social support for partners, but one could speculate that partners have less perceived social support and often feel misunderstood by people in their environment because of the lack of awareness of sarcoidosis as a disease. Previous studies have shown a relationship between social support and the QoL of partners of patients. ${ }^{28,29}$ This needs to be examined in future studies. They may also experience psychological distress, leading to anxiety, ${ }^{17}$ as sarcoidosis can be unpredictable and they worry about the consequences regarding the future of the patient as well as their own future. There is a need for further studies into dyadic coping in sarcoidosis.

In line with other studies, we found a lower QoL for our sarcoidosis patient sample than for healthy controls, especially in the physical health domain, meaning that they feel less physically healthy than the healthy population. ${ }^{13,14,16,30}$ Apart from major organ involvement, sarcoidosis patients can be bothered with reduced muscle strength, loss of physical condition, pain, extreme fatigue, and SFN-associated symptoms. ${ }^{1,31}$ Psychological factors are pervasive, and include anxiety, depressive symptoms and mental fatigue, including memory problems, lack of mental clarity, poor concentration and inability to focus. These factors may not correspond with inflammatory disease activity nor respond to sarcoidosis treatment. ${ }^{1,5}$ The symptoms are disabling, persist after other signs of sarcoidosis activity have resolved, and adversely impact on major life areas, including QoL and work ability. ${ }^{32,33}$ Tan et al. did find that the more symptoms affecting the patient, the more it affected the partner's QoL. ${ }^{34}$

Depressive symptoms, anxiety, and cognitive failure have increasingly been reported in clinical practice as well as in studies. ${ }^{4,6,7,35-38}$ Depressive symptoms are a known predictor of QoL in sarcoidosis. ${ }^{4,38}$ A novel finding is that not only depressive symptoms, but also trait anxiety and subjective cognitive failure predicted QoL in our sarcoidosis sample. The association between cognitive failure and QoL disappeared when we included fatigue and depressive symptoms as mediators (see Figure 9.3A and 9.3B). This may imply multiple competing interpretations. First, cognitive failure presumably results in more depressive symptoms or fatigue, which in turn results in a lower physical QoL. This is in line with a previous study by Hendriks et al. who found that cognitive failure predicted fatigue. ${ }^{5}$ Alternatively, it could indicate that depressive 
symptoms or fatigue causally affect both cognitive failure and physical QoL. Since we also found significant moderating effects between cognitive failure and depressive symptoms as well as fatigue on physical QoL there may also be a moderating mechanism underlying the associations between these variables. This hypothesis was also supported by a previous study by our group in neurosarcoidosis patients, showing fatigue to be a strong predictor of cognitive impairment. ${ }^{39}$ Studies in dialysis patients also found that depressive symptoms predicted cognitive impairment. ${ }^{40,41}$ As follow-up data were lacking in our study and cross-sectional studies generally have limitations with regard to establishing causality, the results have to be interpreted with some caution, and future follow-up studies are warranted to distinguish between these two interpretations for sarcoidosis patients.

To date, studies have shown a high prevalence of fatigue ${ }^{8,12,42-45}$ and SFN-associated symptoms ${ }^{46,47}$ in sarcoidosis patients. In line with previous studies, ${ }^{13-15}$ our study found that sarcoidosis-associated fatigue was negatively associated with overall QoL, as well as the physical and psychological QoL domains, indicating that these QoL domains tend to decrease as patient fatigue increases. Of the partners, only $15 \%$ experienced fatigue (mainly moderate; $30 / 31$ partners) in comparison with $78 \%$ of the sarcoidosis patients. Whether the moderate fatigue in a small proportion of partners might be caused by their own disabilities and/or health problems needs to be explored in future studies. In agreement with earlier studies, a substantial number of our sarcoidosis sample were suffering from SFN-associated symptoms. ${ }^{31,46,47}$ SFN is a very difficult disease to diagnose and to treat. ${ }^{48}$ This causes a lot of uncertainty and frustration for those having to deal with these problems. The presence of SFN-associated symptoms was found to be negatively associated with the physical and environmental QoL domains, indicating that physical and environmental QoL tends to decrease when patients experience more SFN-associated complaints. This could, at least partly, be explained by the fact that patients feel they are not being taken seriously by relatives and friends, and experience misunderstanding because of lack of awareness of sarcoidosis and SFN.

The clinical course of sarcoidosis is highly variable, ranging from spontaneous resolution to disabling chronic disease with various manifestations. ${ }^{49}$ In our cohort, no relation was found between the various manifestations and QoL (data not shown), stressing the importance of assessing not only the organs affected by sarcoidosis but also the non-specific symptoms. ${ }^{5,31}$

All of the above-mentioned non-specific symptoms are not visible in sarcoidosis patients, making it difficult for them to get acknowledged, leading to feelings of 
misunderstanding in many patients and even their partners. ${ }^{17}$ Sarcoidosis patients have reported that QoL is the most important outcome of their treatment and of care, more important than radiographs, pulmonary function tests and blood tests. ${ }^{32}$ Most patients would like to see more attention and support for their psychological problems. ${ }^{17} \mathrm{We}$ also found that patients with better support show a better QoL in all domains, except for physical health. Moreover, although it was beyond the scoop of this study, it would be interesting to evaluate how not living with a partner might influence the QoL of sarcoidosis patients.

Sarcoidosis clinicians usually work in a multidisciplinary team. Our results underline the importance of a multidisciplinary approach assessing not only organ involvement, but any symptoms (organ-related or non-organ-related) and of using a holistic approach with attention to psychological support, for both patients and their partners. One of the possible interventions could be mindfulness-based exercise interventions, which have been found to reduce psychological symptoms and fatigue. ${ }^{50}$

A limitation of the present study was that all patients were recruited in a tertiary referral center. This could have led to selection bias, since it is the more complex patients (in terms of affected organs and symptoms) who are often referred to tertiary referral centers. Hence, our results may not be generalizable to all sarcoidosis patients. However, our results are comparable with those of other QoL studies in sarcoidosis patient samples. Another limitation is that not all partners of the patients with sarcoidosis seen at the outpatient clinic participated in this study, and some patients did not have a partner. However, the sample of sarcoidosis patients whose partners participated did not differ from those without a partner or with a partner who did not participate in our study (except for age). Unfortunately, information on the medical health status of the partners was not collected in this study. It is tempting to speculate that this explains for the lower QoL of the partners compared to healthy controls. Though, the QoL of partners was higher than the QoL of the sarcoidosis patients. Strikingly, however, no relation was found between the QoL of the partners and the burden of the sarcoidosis experienced by the patients themselves indicating that this does not have an impact on the QoL of their partners. One final limitation was the fact that we did not have a matched control group, and that the sample of healthy controls used in our study was rather small, which might increase the influence of sampling error on the estimated statistics in the control sample. Our control group did consist of persons living in the same geographical area/institution and timeframe, and was previously used by our group for similar comparisons in previous studies. ${ }^{13,19,44}$ 


\section{Conclusion}

Couples living with sarcoidosis have a lower QoL than healthy controls. Sarcoidosis patients often struggle with fatigue, SFN-associated symptoms, anxiety, depressive symptoms, and memory and concentration problems. All these symptoms have a negative impact on the QoL of patients, but not on that of their partners. Although these factors pose challenges for objective assessment, they are recognized illnessrelated symptoms meriting formal consideration with validated instruments. Perceived social support positively impacts on the QoL. Further studies are needed to investigate whether offering psychosocial support at the outpatient clinic for sarcoidosis patients as well as their partners would lead to better coping strategies and improve QoL for both of them. 


\section{References}

1 Drent M, Strookappe B, Hoitsma E and De Vries J. Consequences of sarcoidosis. Clin Chest Med 2015;36:727-37.

2 Statement on sarcoidosis. Joint Statement of the American Thoracic Society (ATS), the European Respiratory Society (ERS) and the World Association of Sarcoidosis and Other Granulomatous Disorders (WASOG) adopted by the ATS Board of Directors and by the ERS Executive Committee, February 1999. Am J Respir Crit Care Med 1999;160:736-55.

3 de Kleijn WP, Drent M and De Vries J. Nature of fatigue moderates depressive symptoms and anxiety in sarcoidosis. Br J Health Psychol 2013;18:439-52.

4 Drent $\mathrm{M}$, Wirnsberger RM, Breteler $\mathrm{MH}$, et al. Quality of life and depressive symptoms in patients suffering from sarcoidosis. Sarcoidosis Vasc Diffuse Lung Dis 1998;15:59-66.

5 Hendriks C, Drent M, De Kleijn W, et al. Everyday cognitive failure and depressive symptoms predict fatigue in sarcoidosis: a prospective follow-up study. Respir Med 2018;138S:S24-S30.

6 Hinz A, Brahler E, Mode R, et al. Anxiety and depression in sarcoidosis: the influence of age, gender, affected organs, concomitant diseases and dyspnea. Sarcoidosis Vasc Diffuse Lung Dis 2012;29:139-46.

7 Holas P, Krejtz I, Urbankowski T, et al. Anxiety, its relation to symptoms severity and anxiety sensitivity in sarcoidosis. Sarcoidosis Vasc Diffuse Lung Dis 2013;30:282-8.

8 Jastrzebski D, Ziora D, Lubecki $M$, et al. Fatigue in sarcoidosis and exercise tolerance, dyspnea, and quality of life. Adv Exp Med Biol 2015;833:31-6.

9 Arkema EV, Eklund A, Grunewald J and Bruze G. Work ability before and after sarcoidosis diagnosis in Sweden. Respir Med 2018; 144S:S7-S12.

10 Rice JB, White A, Lopez A, et al. Economic burden of sarcoidosis in a commercially-insured population in the United States. J Med Econ 2017;20:1048-55.

11 Hendriks CMR, Saketkoo LA, Elfferich MDP, et al. Sarcoidosis and work participation: the need to develop a disease-specific core set for assessment of work ability. Lung 2019.

12 Aggarwal AN, Sahu KK and Gupta D. Fatigue and health-related quality of life in patients with pulmonary sarcoidosis treated by oral corticosteroids. Sarcoidosis Vasc Diffuse Lung Dis 2016;33:124-9.

13 Drent $M$, Marcellis R, Lenssen A and De Vries J. Association between physical functions and quality of life in sarcoidosis. Sarcoidosis Vasc Diffuse Lung Dis 2014;31:117-28.

14 Michielsen HJ, Drent M, Peros-Golubicic T and De Vries J. Fatigue is associated with quality of life in sarcoidosis patients. Chest 2006;130:989-94.

15 Michielsen HJ, Peros-Golubicic T, Drent M and De Vries J. Relationship between symptoms and quality of life in a sarcoidosis population. Respiration 2007;74:401-5.

16 Wirnsberger RM, de Vries J, Breteler $\mathrm{MH}$, et al. Evaluation of quality of life in sarcoidosis patients. Respir Med 1998;92:750-6.

17 Moor CC, van Manen MJG, van Hagen PM, et al. Needs, perceptions and education in sarcoidosis: a live interactive survey of patients and partners. Lung 2018;196:569-75.

18 De Kleijn W. Fatigue in sarcoidosis. Edited by. Tilburg: Tilburg University; 2012.

19 Marcellis RG, Lenssen AF, Kleynen S, et al. Exercise capacity, muscle strength, and fatigue in sarcoidosis: a follow-up study. Lung 2013;191:247-56.

20 Development of the world health organization WHOQOL-BREF quality of life assessment. The WHOQOL Group. Psychol Med 1998;28:551-8.

21 De Vries J, Michielsen H, Van Heck GL and Drent M. Measuring fatigue in sarcoidosis: the Fatigue Assessment Scale (FAS). Br J Health Psychol 2004;9:279-91.

22 Hoitsma E, De Vries J and Drent M. The small fiber neuropathy screening list: construction and crossvalidation in sarcoidosis. Respir Med 2011;105:95-100.

23 Broadbent DE, Cooper PF, FitzGerald P and Parkes KR. The Cognitive Failures Questionnaire (CFQ) and its correlates. Br J Clin Psychol 1982;21 (Pt 1):1-16.

24 Van der Ploeg HM, Defares PB, Spielberger CD. A Dutch-Language adaptation of the Spielberger StateTrait Anxiety Inventory. Lisse, The Netherlands: Swets \& Zeitlinger; 1980. 
25 Radloff L. The CESD-D scale: a self-report depression scale for research in the general population. Appl. Psychol. Meas. 1977;1:385-401.

26 De Vries J. The Perceived Social Support Scale, Dutch version. Tilburg, The Netherlands: Tilburg University; 1998.

27 Baron RM and Kenny DA. The moderator-mediator variable distinction in social psychological research: conceptual, strategic, and statistical considerations. J Pers Soc Psychol 1986;51:1173-82.

28 Brand C, Barry L and Gallagher S. Social support mediates the association between benefit finding and quality of life in caregivers. J Health Psychol 2016;21:1126-36.

29 Nightingale CL, Curbow BA, Wingard JR, et al. Burden, quality of life, and social support in caregivers of patients undergoing radiotherapy for head and neck cancer: a pilot study. Chronic Illn 2016;12:236-45.

30 Antoniou KM, Tzanakis N, Tzouvelekis A, et al. Quality of life in patients with active sarcoidosis in Greece. Eur J Intern Med 2006;17:421-6.

31 Voortman M, Hendriks CMR, Elfferich MDP, et al. The burden of sarcoidosis symptoms from a patient perspective. Lung 2019;197:155-161.

32 Baughman RP, Barriuso R, Beyer K, et al. Sarcoidosis: patient treatment priorities. ERJ Open Res 2018; 4.

33 Gerke AK, Judson MA, Cozier YC, et al. Disease burden and variability in sarcoidosis. Ann Am Thorac Soc 2017;14:S421-8.

34 Tan JY, Molassiotis A, Lloyd-Williams M and Yorke J. Burden, emotional distress and quality of life among informal caregivers of lung cancer patients: an exploratory study. Eur J Cancer Care (Engl) 2018; 27.

35 Chang B, Steimel J, Moller DR, et al. Depression in sarcoidosis. Am J Respir Crit Care Med 2001;163:32934.

36 Elfferich MD, De Vries J and Drent M. Type D or 'distressed' personality in sarcoidosis and idiopathic pulmonary fibrosis. Sarcoidosis Vasc Diffuse Lung Dis 2011;28:65-71.

37 Elfferich MD, Nelemans PJ, Ponds RW, et al. Everyday cognitive failure in sarcoidosis: the prevalence and the effect of anti-TNF-alpha treatment. Respiration 2010;80:212-9.

38 Goracci A, Fagiolini A, Martinucci M, et al. Quality of life, anxiety and depression in sarcoidosis. Gen Hosp Psychiatry 2008;30:441-5.

39 Voortman M, De Vries J, Hendriks CMR, et al. Everyday cognitive failure in patients suffering from neurosarcoidosis. Sarcoidosis Vasculitis and Diffuse Lung Diseases 2019;36: 2-10.

40 Zhang YH, Yang ZK, Wang JW, et al. Cognitive changes in peritoneal dialysis patients: a multicenter prospective cohort study. Am J Kidney Dis 2018;72:691-700.

41 Jung S, Lee YK, Choi SR, et al. Relationship between cognitive impairment and depression in dialysis patients. Yonsei Med J 2013;54:1447-53.

42 Bosse-Henck A, Koch R, Wirtz $\mathrm{H}$ and Hinz A. Fatigue and excessive daytime sleepiness in sarcoidosis: prevalence, predictors, and relationships between the two symptoms. Respiration 2017;94:186-97.

43 Drent M, Lower EE and De Vries J. Sarcoidosis-associated fatigue. Eur Respir J 2012;40:255-63.

44 Marcellis RG, Lenssen AF, Elfferich MD, et al. Exercise capacity, muscle strength and fatigue in sarcoidosis. Eur Respir J 2011;38:628-34.

45 Strookappe B, De Vries J, Elfferich M, et al. Predictors of fatigue in sarcoidosis: the value of exercise testing. Respir Med 2016;116:49-54.

46 Bakkers M, Merkies IS, Lauria G, et al. Intraepidermal nerve fiber density and its application in sarcoidosis. Neurology 2009;73:1142-8.

47 Hoitsma E, Drent M, Verstraete $E$, et al. Abnormal warm and cold sensation thresholds suggestive of small-fibre neuropathy in sarcoidosis. Clin Neurophysiol 2003;114:2326-33.

48 Voortman M, Fritz D, Vogels OJM, et al. Small fiber neuropathy: a disabling and underrecognized syndrome. Curr Opin Pulm Med 2017;23:447-57.

49 Schupp JC, Freitag-Wolf S, Bargagli E, et al. Phenotypes of organ involvement in sarcoidosis. Eur Respir J 2018;51.

50 Saketkoo LA, Karpinski A, Young J, et al. Feasibility, utility and symptom impact of modified mindfulness training in sarcoidosis. ERJ Open Res 2018;4. 


\section{Online supplement questionnaires}

1. The WHOQoL-Bref is a generic, cross-culturally developed comprehensive measure of QoL. It consists of 24 questions in four domains (physical health, psychological health, social relationships and environment) related to QoL and two questions that assess overall QoL and general health. ${ }^{18}$ The response scale is a five-point scale (1=very poor/not at all/very dissatisfied/ never, to 5=very good/extreme amount/extremely/completely/very satisfied/always). Higher score indicates a better Qol.

2. The FAS is a 10-item self-report fatigue questionnaire. In addition to the total fatigue score, the FAS yields a mental fatigue score and a physical fatigue score. The response scale is a five-point scale (1=never, to 5 =always; range 10-50). A score $>22$ indicates fatigue and a score $>34$ indicates extreme fatigue. The FAS shows good reliability and validity in measuring the fatigue of sarcoidosis patients. ${ }^{19}$

3. The SFNSL is a 21-item self-administered questionnaire to screen for symptoms related to SFN. The response scale is a five-point scale ( $0=n e v e r / n o t$, to 4=always/severe; range 0 to 84). The cut-off score for the SFNSL is 11: a score below 11 indicates few or no SFN-related symptoms (normal temperature threshold testing [TTT]), while a score of 11-48 indicates probable or highly likely SFN, and a score above 48 is indicative of SFN (abnormal TTT). ${ }^{20}$

4. The CFQ is a self-report questionnaire consisting of 25 items assessing deficits regarding attention, perception, memory and motor functioning in everyday life. The response scale is a five-point scale ( $0=$ never, to $4=$ very often; range $0-100)$. A higher total score indicates more subjective cognitive failure. A high CFQ score is defined as a score $\geq 43$. $^{21}$

5. The STAI concerns differences between individuals in their disposition to respond to stressful situations with varying amounts of stress. This scale consists of 20 statements, asking patients to describe how they generally feel. The response scale is a four-point scale ( $1=$ not at all, to $4=$ very much so; range 20-80). A higher score indicates more anxiety. The psychometric characteristics of the Dutch version of this questionnaire are well established and considered good. ${ }^{22}$ High trait anxiety was defined as a score above 40 , based on Dutch norm scores. ${ }^{22}$

6. The CES- $D^{23}$ is a 20-item scale designed to measure the presence and degree of depressive symptoms. Scores on the CESD can range from 0-60, in which a higher score indicates more depressive symptoms. The response scale is a four-point scale ( $0=$ rarely/never, to $3=$ mostly/always; range $0-60)$. Scores $\geq 16$ are indicative of a depressive disorder. Reliability and criterion validity appear to be good. ${ }^{50}$ 
7. The PSSS12 was used, concerning 12 items. The total score of the 12 -item version of the PSSS was used to assess general perception of social support. The response scale is a seven-point scale (1=very strongly disagree, to 7=very strongly agree; range 12-84). The PSSS has good reliability and validity in patients undergoing coronary angiography, ${ }^{51}$ and has been translated into Dutch. ${ }^{24}$ 


\section{Chapter 10}

Summary and general discussion 



\section{Summary}

Sarcoidosis is a multisystem inflammatory disorder characterized by the formation of non-caseating granulomas in various organ systems. ${ }^{1,2}$ The lymphatic system and the lungs are most commonly affected, but any organ can be involved. Involvement of the nervous system, or neurosarcoidosis, is detected in $5 \%$ of cases during life, ${ }^{3-6}$ although autopsy studies have shown neurologic involvement in $25-50 \%^{7,8}$ Although the pathogenesis of sarcoidosis has not been fully elucidated, environmental (i.e. infectious, metal, silica, silicones, ink) and genetic factors may contribute substantially to its pathogenesis, involving an exaggerated granulomatous reaction to a trigger.

Sarcoidosis occurs in men and women throughout the world, affecting all races and any people aged between 25 and 45 years who have a predisposition. ${ }^{2}$ The influence of race and gender on the epidemiology of sarcoidosis is generally recognized. The incidence of sarcoidosis in patients of African descent is high compared to that in Caucasians. ${ }^{9}$ Moreover, they often present with more severe organ involvement, a worse prognosis and higher mortality rates than Caucasians. ${ }^{9-11}$ It has also been acknowledged that the prevalence of various organ manifestations differs between different ethnic subgroups, e.g. cutaneous and ocular sarcoidosis is more common in patients of African descent than in Caucasians, while hypercalcemia is more common in Caucasians. ${ }^{4}$ In terms of gender differences, pulmonary and cardiac sarcoidosis is more common in men, whereas peripheral lymph node involvement, cutaneous, ocular and liver sarcoidosis are seen more often in women. ${ }^{4,12}$

The clinical manifestation, natural history and prognosis of sarcoidosis are highly variable, and its course is often unpredictable. ${ }^{1}$ Depending on the organs involved and the severity of the granulomatous inflammation, patients present with a broad range of symptoms. In addition to organ-related symptoms, patients often suffer from disabling non-specific, non-organ-related symptoms, ${ }^{13-15}$ such as fatigue, depressive symptoms, pain, anxiety and cognitive failure. All these symptoms have an impact on the quality of life (QoL). ${ }^{13,16-22}$

This thesis is divided into two parts:

The aims of the studies in the first part of this thesis were to investigate the prevalence of the different neurosarcoidosis manifestations and the influence of race and gender on this. Furthermore, we aimed to determine the prevalence of cognitive failure in these patients.

The aims of the studies in the second part were to describe the burden of non-specific symptoms (small fiber neuropathy (SFN), fatigue, cognitive failure, depressive 
symptoms, and anxiety) in sarcoidosis patients and the impact on the QoL of these patients and their partners.

\section{Overview of the main findings}

Chapter 1, the general introduction, provides a summary of the epidemiology and pathogenesis of sarcoidosis, the clinical presentation of neurosarcoidosis, and nonspecific symptoms. In addition, it presents a summary of treatment options of neurosarcoidosis and describes the non-specific symptoms of fatigue and SFN.

Chapter 2 presents an overview of the literature regarding neurosarcoidosis. The clinical presentation is heterogeneous. Most patients present with cranial nerve palsy, headache and sensory abnormalities. Patients are classified according to the probability of the diagnosis using the modified Zajicek criteria. ${ }^{23} \mathrm{~A}$ crucial feature in these criteria is histopathological confirmation of non-caseating granulomas in affected tissue outside the nervous system. Radiological abnormalities on neuroimaging are non-specific. No biomarkers have been described that effectively identify patients with sarcoidosis. Despite the use of new therapies, a third of the patients remain unchanged, deteriorate or die. Diagnosing and treating patients with neurosarcoidosis remains a challenge. Long-term prospective studies evaluating patients suspected of neurosarcoidosis are needed to assess the sensitivity and specificity of ancillary investigations and the diagnostic criteria. Furthermore, studies are needed to evaluate the prognosis and the optimal treatment strategy.

Chapter 3 investigates neurosarcoidosis manifestations and the influence of race and gender, as well as the treatment strategies. In a multicenter observational cohort study, we included 194 neurosarcoidosis patients from two tertiary referral centers; the ILD Center of Excellence of the St. Antonius Hospital, Nieuwegein and the Amsterdam University Medical Centre (UMC), both in the Netherlands, as well as patients included in the Dutch Neurosarcoidosis Registry. The most common neurosarcoidosis manifestations were chronic meningitis (41\%), cranial nerve dysfunction (39\%), myelopathy $(28 \%)$, and cerebral parenchymal disease $(26 \%)$. There were no differences in age, gender and race between the various manifestations, except for peripheral polyneuropathy, which was more prevalent in men than in women (21\% versus $10 \%)$. Most neurosarcoidosis patients were treated with prednisone $(89 \%)$, methotrexate (46\%), or infliximab (21\%). A total of 102 patients (53\%) received second- or third-line treatment. Over half of the patients required multiple immunosuppressive treatments. 
Chapter 4 presents an evaluation of the cognitive failure rate in neurosarcoidosis patients compared to a general sarcoidosis sample. Cognitive failure is characterized by memory and concentration problems. Everyday cognitive failure was assessed using the Cognitive Failure Questionnaire (CFQ). A cross-sectional web-based survey was conducted in a national sample of 131 neurosarcoidosis patients. We found that the mean CFQ score was significantly higher in the neurosarcoidosis sample $(45.6 \pm 20.7)$ compared to the general sarcoidosis sample $(36.2 \pm 15.9 ; \mathrm{p}<0.0001)$. High CFQ scores $(\geq 43)$ were found in $55.7 \%$ (neurosarcoidosis) and $33.9 \%$ (general sarcoidosis) $(p<0.0001)$. The fatigue assessment scale (FAS) score (OR 21.4) and the score on the small fiber neuropathy screenings list (SFNSL) (OR 4.3) were the strongest positive predictors of a high CFQ score. Cognitive failure is a significant problem in neurosarcoidosis.

Chapter $\mathbf{5}$ provides an overview of the current literature regarding the management of neurosarcoidosis, including SFN. The clinical manifestation and course of neurosarcoidosis are particularly variable. Although neurosarcoidosis occurs in up to ten percent of sarcoidosis patients, it can lead to significant morbidity and some mortality. Recent guidelines have helped to clarify criteria for diagnosing neurosarcoidosis. No firm guidelines exist for whether, when, and how treatment should be started. Treatment depends on the presentation and distribution, extensiveness, and severity of neurosarcoidosis. As regards evidence-based treatment, only a few randomized controlled trials have been done. Hence, several aspects of sarcoidosis and neurosarcoidosis management are not fully addressed by the current literature. A multidisciplinary approach to the management of sarcoidosis is strongly recommended.

Chapter 6 provides an overview of the current literature regarding SFN, which causes high morbidity with disabling symptoms and impact on QoL. Patients may benefit from being diagnosed with SFN, even if no underlying cause is identified and no specific treatment is yet available. Clinical diagnostic criteria have been proposed, but no gold standard exists, and each test has its limitations. The diagnosis requires a combination of typical symptoms, abnormal neurologic findings, and absence of large fiber involvement. Clinicians should be aware of overlapping symptoms of SFN and fibromyalgia. Treatment is often difficult, even when the underlying cause is identified and appropriately treated. Usually, only symptomatic relief of complaints is available. Awareness of SFN and related symptoms is of great clinical relevance. Guidelines for appropriate diagnostic workup using a stepwise approach involving a combination of tests are warranted. 
Chapter 7 reports on the minimal important difference (MID) of the SFNSL. The MID is the smallest change in a score that a patient perceives to be important. Previous studies found that $40-60 \%$ of the sarcoidosis patients suffer from SFN, substantially affecting QoL. The usefulness of any questionnaire in clinical management and research trials depends on its interpretability, requiring the smallest detectable change (SDC) and minimal important difference (MID) to be known. Patients with neurosarcoidosis and/or sarcoidosis-associated SFN symptoms $(n=138)$ included in the online Dutch Neurosarcoidosis Registry participated in a prospective, longitudinal study. The SFNSL was completed both at baseline and at 6 -months' follow-up by 89 patients. The MID for the SFNSL is 3.5 points for a clinically relevant change over a 6 -month period. The MID can be used in the follow-up and management of SFN-associated symptoms in patients with sarcoidosis, though with some caution, as the SDC was found to be higher.

Chapter 8 evaluates the burden of sarcoidosis symptoms from a patient perspective in three European countries. The clinical manifestations of sarcoidosis vary widely, depending on the intensity of the inflammation and the organ systems affected. A cross-sectional web-based anonymous survey about complaints (organ-related as well as non-specific, non-organ-related) was conducted among sarcoidosis patients in Denmark, Germany, and the Netherlands. The questionnaire was completed by 1072 sarcoidosis patients (152 Danish, 532 German, and 388 Dutch). Almost all patients reported having sarcoidosis-associated symptoms (organ-related as well as nonspecific, non-organ-related). Fatigue was reported by almost all respondents (90\%), followed by symptoms associated with SFN (86\%), and pulmonary symptoms (72.4\%). More than $50 \%$ of the respondents were being treated with prednisone, a finding which was comparable for all three countries. In contrast, second- and third-line treatment differed substantially between Denmark, Germany, and the Netherlands. Sarcoidosis patients in Denmark, Germany, and the Netherlands present with similar self-reported symptoms, organ-related as well as non-specific, non-organ-related.

Chapter 9 reports on a study of QoL in sarcoidosis patients and their partners. Consequences of sarcoidosis are wide-ranging and the symptom burden has a great impact on patients' QoL. However, the QoL of couples living with sarcoidosis has not yet been studied. Sarcoidosis outpatients, recruited at Maastricht University Medical Center $(n=443)$, and their partners $(n=208)$ completed several questionnaires, including the World Health Organization Quality of Life - BREF (WHOQOL-BREF), FAS, SFNSL, and CFQ. The QoL of the partners as well as of the sarcoidosis patients was reduced compared with healthy controls, though that of partners was less reduced than that of the patients, especially regarding the physical health domain. All non-specific 
symptoms studied, as well as perceived social support, predicted one or more QoL domains in the sarcoidosis patients, but these factors did not predict the QoL of their partners. In the management of sarcoidosis is it important to focus not only on the patients, but also on their partners.

\section{Discussion}

Sarcoidosis is a complex, multisystem disorder of unknown cause(s) that imposes a burden on patients' lives. ${ }^{24,25}$ The clinical presentation of sarcoidosis is highly variable and the course of the disease is unpredictable. In addition to specific organ-related symptoms causing functional impairments and symptoms associated with comorbidities, sarcoidosis patients are disabled by less specific symptoms, including fatigue. ${ }^{9,25,26}$ All symptoms may have a significant influence on patients' daily activities and their social and professional lives. Many different clinical phenotypes of sarcoidosis exist; no two sarcoidosis patients are the same. Therefore, it is hard to develop a general diagnostic or treatment protocol for sarcoidosis patients. Currently, 20 years after the publication of the first guidelines ${ }^{1}$ a new attempt has been started to update the diagnostic work-up and therapeutic options for sarcoidosis.

\section{Neurosarcoidosis}

Like sarcoidosis itself, the presentation of neurosarcoidosis is variable. Furthermore, diagnosing neurosarcoidosis remains a clinical challenge, due to its diversity and the absence of specific biomarkers. In most cases of suspected neurosarcoidosis, nervous system histology is not feasible and other biopsy sites outside the nervous system should be searched for. Moreover, it is important to rule out other causes, perform a complete work-up for suspected sarcoidosis, and use the diagnostic criteria established by Stern et al. to enable possible, probable, or definite neurosarcoidosis to be diagnosed (see Table 10.1). ${ }^{27}$ 
Table 10.1 Proposed diagnostic criteria for neurosarcoidosis. ${ }^{27}$

\begin{tabular}{|c|c|c|}
\hline Possible & Probable & Definite \\
\hline $\begin{array}{l}\text { 1. the clinical presentation and } \\
\text { diagnostic evaluation suggest } \\
\text { neurosarcoidosis, as defined by } \\
\text { the clinical manifestations, MRI, } \\
\text { CSF and/or EMG/NCS findings } \\
\text { typical of granulomatous } \\
\text { inflammation of the nervous } \\
\text { system, and after rigorous } \\
\text { exclusion of other causes. }\end{array}$ & $\begin{array}{l}\text { 1. the clinical presentation and } \\
\text { diagnostic evaluation suggest } \\
\text { neurosarcoidosis, as defined by } \\
\text { the clinical manifestations, MRI, } \\
\text { CSF and/or EMG/NCS findings } \\
\text { typical of granulomatous } \\
\text { inflammation of the nervous } \\
\text { system, after rigorous exclusion } \\
\text { of other causes. }\end{array}$ & $\begin{array}{l}\text { 1. the clinical presentation and } \\
\text { diagnostic evaluation suggest } \\
\text { neurosarcoidosis, as defined by } \\
\text { the clinical manifestations, MRI, } \\
\text { CSF and/or EMG/NCS findings } \\
\text { typical of granulomatous } \\
\text { inflammation of the nervous } \\
\text { system, after rigorous exclusion } \\
\text { of other causes. }\end{array}$ \\
\hline $\begin{array}{l}\text { 2. there is no pathologic } \\
\text { confirmation of granulomatous } \\
\text { disease. }\end{array}$ & $\begin{array}{l}\text { 2. there is pathologic confirmation } \\
\text { of systemic granulomatous } \\
\text { disease consistent with } \\
\text { sarcoidosis. }\end{array}$ & $\begin{array}{l}\text { 2. the nervous system pathology is } \\
\text { consistent with } \\
\text { neurosarcoidosis. } \\
\text { type a. Extra-neural } \\
\text { sarcoidosis is evident. } \\
\text { type b. No extra-neural } \\
\text { sarcoidosis is evident } \\
\text { ("isolated CNS sarcoidosis"). }\end{array}$ \\
\hline
\end{tabular}

Most manifestations of neurosarcoidosis are very debilitating and regularly lead to residual damage and high relapse rates, despite immunosuppressive treatment. That is why it is essential to raise awareness among practitioners, so that neurosarcoidosis is recognized and treated without delay to hopefully limit or prevent residual damage.

Treatment is almost always warranted in neurosarcoidosis. However, the treatment regime is not the same in all neurosarcoidosis patients. The intensity of treatment should depend on the severity of the manifestation and the burden to the patient. For example, a facial nerve dysfunction can often be treated with prednisone monotherapy or no treatment at all, whereas spinal cord disease should be treated without delay with second- (methotrexate, azathioprine) or third-line treatment (tumour necrosis factor-alpha [TNF- $\alpha$ ] antagonists) (see chapters 2 and 5). In line with others, we found that in our neurosarcoidosis sample, more than half of the patients needed second- or third-line treatment (see chapter 3). ${ }^{28-33}$ Chapters 2 , and 5 discuss guidelines for ways to treat the various manifestations of neurosarcoidosis. To date, personalized medicine is mandatory in all sarcoidosis and neurosarcoidosis patients, guided by multidisciplinary discussions. However, to offer the clinician some help, Figure 10.1 presents a proposed treatment algorithm. 


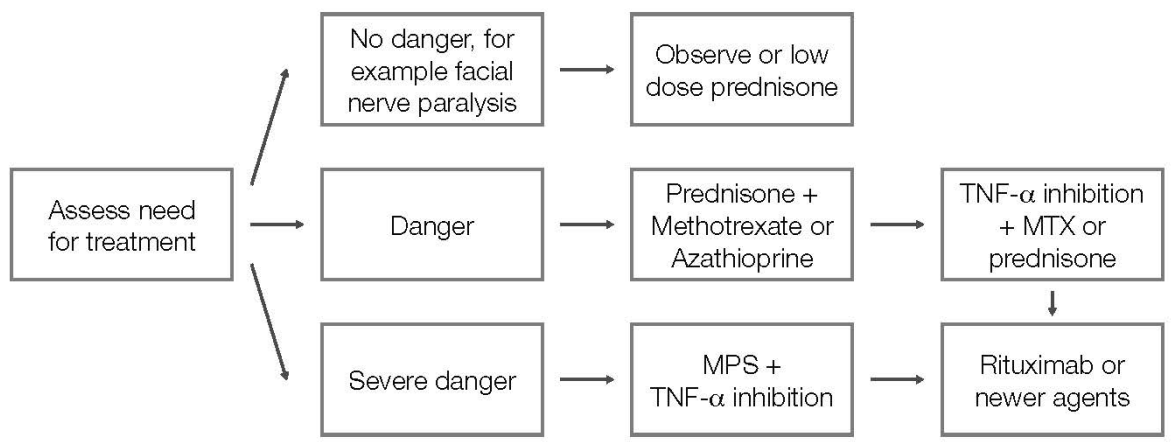

Figure 10.1 Proposed treatment algorithm in neurosarcoidosis.

MPS=methylprednisone; MTX=methotrexate; TNF- $\alpha=$ tumour necrosis factor-alpha

The difficulty in performing research into this extremely rare disease is that the samples studied are mostly small, especially when investigating each of the neurosarcoidosis manifestations separately. Due to the rarity of this disease, a randomized controlled trial (RCT) is almost never a realistic option. Many recommendations developed for neurosarcoidosis are therefore based on observational studies and experts' opinions. The research field is, however, currently shifting from RCTs to more real-world evidence. ${ }^{34,35}$ RCTs have a strong internal validity, but overall a weaker external validity and are therefore mostly not generalizable to clinical practice for individual patients. Moreover, sarcoidosis patients are mostly not eligible for RCTs, due to comorbidities. Observational studies and precision-based (biomarker) studies can be a good or maybe even better alternative, especially for the patients. Larger, multicenter observational and biomarker studies are therefore the best option for research in this field (see the section entitled Future Studies).

\section{Non-specific, non-organ related symptoms}

Sarcoidosis is a burdensome disease, due to organ-related as well as non-organ-related, non-specific symptoms, such as those associated with small fiber neuropathy (SFN), fatigue or cognitive failure. These latter symptoms are highly prevalent, as shown in chapters 4 and 8 of this thesis, leading to a high burden. Since these symptoms are not visible and hard to objectify/diagnose, many patients feel misunderstood and not taken seriously. Hence, it is essential to identify all disabling symptoms, as this will ultimately enable healthcare providers, supported by researchers, to tailor interventions to individual patients and improve their quality of life (QoL). Given the wide variety of 
symptoms, not limited to one discipline, and uncertainties regarding pathophysiology and possible causes, a multidisciplinary approach focusing on somatic as well as psychosocial aspects is recommended for this erratic disorder.

\section{Diagnostic approach}

Questionnaires, such as the Fatigue Assessment Scale (FAS) and the Small Fiber Neuropathy Screening List (SFNSL), have been developed to assess these complaints in sarcoidosis patients and quantify them. They are therefore useful to assess these nonspecific symptoms, but they are not meant for diagnosing, and care providers should be aware of this. For example, the SFNSL should be used as a screening tool for SFN, and in case of a high score the patient should be referred to a neurologist for further evaluation and exclusion of other causes. Diagnosing SFN remains challenging, since a gold standard is lacking. Various diagnostic criteria have been proposed, but no set of well-defined criteria is as yet available. All diagnostic modalities (quantitative sensory testing, nociceptive evoked potentials, skin wrinkling, skin biopsy, corneal confocal microscopy) have their limitations, although skin biopsy is considered by some to be the 'silver standard'. ${ }^{36-39}$ However, a normal skin biopsy does not completely exclude SFN, probably due to its patchy distribution in sarcoidosis patients. Moreover, skin biopsy does not always correlate with complaints. ${ }^{40,41}$ Corneal confocal microscopy has high potential, since it can detect early nerve damage and a correlation has been established between the severity of neuropathy and progressive corneal nerve degeneration. ${ }^{42-45}$ However, further research is necessary to establish a control group. A pilot study into cognitive failure was performed by our group, examining standard neuropsychological tests that were used to assess the cognitive domains of memory sensorimotor speed, information processing speed and cognitive flexibility. It found that cognitive failure did not imply cognitive impairment. Thus, subjective cognitive failure was not associated with cognitive impairment. ${ }^{14}$

\section{Treatment options}

Previous studies have found that most of the non-specific symptoms are interrelated ${ }^{13,14,46}$ (see also chapter 4), so specifically treating one symptom can help reduce other non-specific symptoms as well. For example, as might be expected, it has been shown that treating fatigue also resulted in an improvement of cognition. ${ }^{47}$

Symptoms of sarcoidosis-associated SFN are often disabling and difficult to treat. Usually, only symptomatic relief of complaints is achieved with neuropathic pain killers. Other options could be immunoglobulins (IVIG), TNF- $\alpha$ antagonists or Cibinetide ${ }^{\circledR}$. The 
largest case series for IVIG $(n=76)$ suggests that up to $75 \%$ of patients will derive symptomatic benefit, but these results are limited by the absence of a defined standard for assessing treatment response, as well as by patient selection bias, differences in concomitant treatment regimens, and lack of a placebo group. ${ }^{48}$ The putative mechanism for the effectiveness of IVIG is unclear but may relate to immunomodulatory effects. TNF- $\alpha$ is known to reduce mechanical nociceptive thresholds ${ }^{49,50}$ and has been found in higher concentrations in distal skin biopsies of patients with length-dependent SFN. ${ }^{51}$ The monoclonal TNF- $\alpha$ antagonist infliximab has been assessed in a retrospective cohort $(n=26)$. Approximately $70 \%$ of patients experienced a reduction of SFN-associated symptoms, although the magnitude of the effect is difficult to ascertain from the available data. ${ }^{48}$ Response rates for TNF- $\alpha$ antagonists may relate to the degree of TNF- $\alpha$ suppression, since the GG promoter variant, associated with less intensive TNF transcription, was also associated with better outcomes in treated patients. ${ }^{52}$ Cibinetide $^{\circledR}$, previously known as ARA-290, is an innate repair receptor agonist that has anti-inflammatory and neuroprotective properties. $^{53-55}$ Multiple randomized, placebo-controlled, double-blind studies have previously shown effects of Cibinetide ${ }^{\circledR}$ in reducing SFN-associated symptoms and increasing nerve fiber density (skin and cornea). ${ }^{56-58}$ It is disappointing that a drug that offers such benefits to patients is not yet on the market, and the question remains whether it will ever be.

A recently published placebo-controlled study $(n=47)$ showed an effect of lacosamide on pain intensity in patients with SFN due to voltage-gated channel mutations (mutations in SCN9A, encoding Nav1.7). ${ }^{59}$ Lacosamide is an anticonvulsant that acts on Nav1.3, Nav1.7, and Nav1.8. Perhaps this could also be an option for the treatment of neuropathic pain in sarcoidosis-associated SFN, although the pathophysiological mechanism is probably different. A recent study examined the effect of virtual reality (VR) technology on the recovery of the autonomic nervous system in volunteers affected by stress. ${ }^{60}$ This might be an option in treating autonomic dysfunction in sarcoidosis-associated SFN. It is, however, still hard to recommend any treatment, because the evidence is not compelling and no guideline for the treatment of sarcoidosis-associated SFN yet exists.

\section{Practical implications and directions for future studies}

As stated above, sarcoidosis is a heterogeneous disease with a variety of clinical symptoms, organ-related as well as non-specific, non-organ-related. A complete evaluation of sarcoidosis could make use of a panel with four disease domains or dimensions: extent of disease, severity, activity, and impact. ${ }^{13}$ Severity of sarcoidosis in 
each organ is defined as the degree of organ damage sustained from sarcoidosis. The interpretation of the severity of sarcoidosis can be complicated by its heterogeneity. In the case of neurosarcoidosis, the most important aspect is awareness of the presenting symptoms and manifestations of neurosarcoidosis. This knowledge could enable earlier recognition of these manifestations and earlier introduction of treatment. Treatment is warranted for almost all patients with neurosarcoidosis.

The burden of non-specific, non-organ-related symptoms in sarcoidosis patients, but also in their caregivers/partners, is high. In addition to organ-related complaints and functional disorders, fatigue, pain, energy loss, and concentration and memory problems affect whether or not someone is capable of performing work and to what extent. A large percentage of sarcoidosis patients feel that their complaints were not taken seriously when their claims for work capacity were evaluated. ${ }^{61} \mathrm{~A}$ review by Barth et al. ${ }^{62}$ demonstrated considerable variation in judgements on work disability among medical examiners, which probably has to do with the limited knowledge that clinicians generally possess about sarcoidosis as a disease with extra-pulmonary involvement.

Since these problems are frequent and may have a major influence on patients' daily activities, their social and professional lives and their QoL, it is important to recognize and quantify these problems in the evaluation of sarcoidosis patients. ${ }^{15}$ There should be a greater degree of understanding for this, and patients should feel that they are being taken seriously and are a partner in the management of their disease.

The above non-specific symptoms, including pain, are even more prevalent in neurosarcoidosis patients (see chapter 4). Pain, a complex phenomenon influenced by a range of genetic, biological, psychological and social factors, is a major component of SFN, and is the result of physiological interactions between central and peripheral nervous system signaling. The many different aspects of pain mean that neurologists, pulmonologists and other clinicians need to have enough expertise to diagnose the type of pain correctly and treat it appropriately. For both clinicians and patients, it is a complex disease, which should be treated by sarcoidosis specialists with experience in all sarcoidosis symptoms. Moreover, absence of evidence does not mean evidence of absence.

We recommend standardized assessment of non-specific symptoms (fatigue, SFNassociated symptoms, cognitive failure, depressive symptoms and anxiety), with validated instruments, in the work-up of sarcoidosis patients. This assessment is important in the management of these patients in the outpatient clinic but also for the evaluation of their capacity for work. Management of sarcoidosis requires a 
multidisciplinary personalized approach that focuses on somatic as well as psychosocial aspects of the disease. When assessing individuals during an occupational disability assessment, the nature and severity of the condition, and especially the consequences for the specific patient, must be taken into account in the advice.

The rarity of sarcoidosis makes the care for these patients extra challenging, even more so in ultra-rare manifestations such as neurosarcoidosis. Future studies are warranted to enhance our understanding of the diversity of neurosarcoidosis, the determinants of its morbidity, severity, and mortality, as well as optimal treatment regimens, in order to determine the best treatment and to improve the quality of life of patients suffering from this rare form of sarcoidosis.

\section{Diagnostic work-up}

As mentioned above, neurosarcoidosis is a serious manifestation of sarcoidosis with generally a worse prognosis. Future research should focus on optimizing the diagnostic work-up of neurosarcoidosis and on establishing whether biomarkers such as TNF- $\alpha$ polymorphisms or other proteins (for example S100B, a calcium-binding protein) may be valuable diagnostic tools. Future studies regarding non-specific symptoms are also needed. Non-specific symptoms are difficult to objectify. Previously, the FAS and SFNSL were developed and validated to objectify fatigue and SFN-associated symptoms, respectively, in sarcoidosis. Several questionnaires also exist for cognitive failure, depressive symptoms and anxiety, which need to be validated in sarcoidosis in future studies.

A previous study by Hendriks et al. found a relation between fatigue and cognitive failure. ${ }^{14}$ Since all of the non-specific symptoms seem to be interrelated, it is reasonable to look for a common pathway and treat this accordingly, in order to treat all nonspecific symptoms.

Regarding SFN, research should focus on identifying and understanding the pathological basis of SFN, which will facilitate further studies on diagnostic methods and novel treatment approaches. Prospective research is warranted to improve the diagnostic work-up (for example by establishing a control group for the corneal confocal microscopy and formulating/validating diagnostic criteria), treatment, and outcome. Furthermore, research is needed specifically with regard to autonomic dysfunction in patients with SFN-associated symptoms. 


\section{Treatment}

Currently, no treatment exists for sarcoidosis. Each sarcoidosis patient is unique, and what works for one person may not work for someone else. Therefore, future research should focus on the most effective treatment for sarcoidosis and neurosarcoidosis, considering various phenotypes and genotypes. Moreover, we should focus on treatment modalities beyond prednisone (quick effect, fewer side-effects) as the firstline treatment for sarcoidosis, since prednisone can cause many adverse side-effects and imposes a great burden on patients. Multiple more recent agents are currently being studied in Phase I and II trials.

As regards fatigue, short-term benefits of physical therapy/training have been found in previous studies. Long-term benefits of these interventions should be investigated in larger randomized controlled studies.

\section{Guidelines}

As mentioned above, non-specific, non-organ-related symptoms in sarcoidosis are disabling and difficult to objectify and treat. It is important to pay attention to these symptoms in sarcoidosis patients as well, in addition to the organ-related symptoms, and to also address this in national and international guidelines. To this end, more knowledge should be acquired by various organizations, including the Dutch association of physicians for pulmonary diseases and tuberculosis (NVALT) and the World Association of Sarcoidosis and other Granulomatous Disorders (WASOG), but also organizations such as the Dutch institute for employee insurance (UWV). Sarcoidosis is an erratic disease that presents differently in each patient. When assessing individuals, such as during an occupational disability assessment, the nature and severity of the condition, and especially the consequences for that specific patient, must be taken into account in the advisory report on work capacity.

The development of a disease-specific core set for sarcoidosis under the framework of the International Classification of Functioning, Disability and Health (ICF), ${ }^{63}$ with input from patients as well as physicians, rehabilitation specialists, specialist nurses, and so on, could be a first step in examining and structuring the multi-faced functional impact of sarcoidosis on employment. Further studies comparing the evaluation of disability as graded by the medical staff and the patients themselves would be very interesting. Are patients themselves capable of grading their own disability, and are the health care workers able to grade disability without objective parameters? Are care providers biased in making their final decision due to a lack of objective parameters? This is a very challenging problem, and should be evaluated in future studies. We believe that the 
advantage would be that patients are involved in the management process, and their own judgments can be incorporated in the decision making. Patients want nothing more than to participate in social life again, but are highly frustrated that they simply are temporarily unable to make the most of their lives.

Last but not least, we believe it is very important to incorporate the patients themselves in developing new research questions and performing research, since, after all, they are the ones for whom we are doing all the research.

\section{Key issues}

- Neurosarcoidosis is a serious and highly heterogeneous manifestation of sarcoidosis.

- More than half of patients with neurosarcoidosis need second- (methotrexate, azathioprine, mycophenolate mofetil) or third-line (TNF- $\alpha$ inhibition) treatment to control the disease, the exception being patients with a facial nerve dysfunction.

- Sarcoidosis patients suffer not only from organ-related symptoms, but also from a wide spectrum of rather nonspecific disabling symptoms, which are common $(>75 \%)$, such as cognitive problems, fatigue, depressive symptoms, anxiety, and SFN-associated symptoms

- The SFNSL is a useful questionnaire to assess SFN-related symptoms, but is not intended to diagnose SFN.

- Cognitive problems are even more prevalent in neurosarcoidosis patients than in the general sarcoidosis patient population (55\% versus $33 \%$ ).

- Management of sarcoidosis requires a multidisciplinary personalized approach that focuses on somatic as well as psychosocial aspects of the disease.

- Patient care should not be limited to patients but also include their caregivers/partners, since the latter also experience a reduced QoL.

- Different clinical phenotypes of sarcoidosis exist; no two sarcoidosis patients are the same. Therefore, it is hard to develop a general diagnostic or treatment protocol for sarcoidosis patients.

- Future research should focus on optimizing the diagnostic work-up in terms of imaging and biomarkers, on furthering our understanding of all aspects of this disease, and on developing new therapeutic strategies. 


\section{References}

1 Statement on sarcoidosis. Joint Statement of the American Thoracic Society (ATS), the European Respiratory Society (ERS) and the World Association of Sarcoidosis and Other Granulomatous Disorders (WASOG) adopted by the ATS Board of Directors and by the ERS Executive Committee, February 1999. Am J Respir Crit Care Med 1999; 160:736-55.

2 Grunewald J, Grutters JC, Arkema EV, et al. Sarcoidosis. Nat Rev Dis Primers 2019;5(1):45.

3 Fritz D, van de Beek D and Brouwer MC. Clinical features, treatment and outcome in neurosarcoidosis: systematic review and meta-analysis. BMC Neurol 2016; 16:220.

4 Judson MA, Boan AD and Lackland DT. The clinical course of sarcoidosis: presentation, diagnosis, and treatment in a large white and black cohort in the United States. Sarcoidosis Vasc Diffuse Lung Dis 2012; 29:119-27.

5 Baughman RP, Culver DA, Judson MA, et al. Self-reported organ involvement in sarcoidosis: results of a multinational registry. American Journal of Respiratory and Critical Care Medicine 2018; 197:A7482.

6 Ungprasert P, Crowson CS and Matteson EL. Characteristics and long-term outcome of neurosarcoidosis: a population-based study from 1976-2013. Neuroepidemiology 2017; 48:87-94.

7 Iannuzzi MC, Rybicki BA, Teirstein AS. Sarcoidosis. N Engl J Med 2007; 357:2153-65.

8 Joseph FG, Scolding NJ. Sarcoidosis of the nervous system. Pract Neurol 2007; 7:234-44.

9 Gerke AK, Judson MA, Cozier YC, et al. Disease burden and variability in sarcoidosis. Ann Am Thorac Soc 2017; 14:S421-S428.

10 Kirkil G, Lower EE, Baughman RP. Predictors of mortality in pulmonary sarcoidosis. Chest 2018; 153:105113.

11 Mirsaeidi M, Machado RF, Schraufnagel D, et al. Racial difference in sarcoidosis mortality in the United States. Chest 2015; 147:438-449.

12 Ungprasert P, Crowson CS, Matteson EL. Influence of gender on epidemiology and clinical manifestations of sarcoidosis: a population-based retrospective cohort study 1976-2013. Lung 2017; 195:87-91.

13 Drent M, Strookappe B, Hoitsma E, De Vries J. Consequences of sarcoidosis. Clin Chest Med 2015; 36:727-37.

14 Hendriks C, Drent M, De Kleijn et al. Everyday cognitive failure and depressive symptoms predict fatigue in sarcoidosis: a prospective follow-up study. Respir Med 2018; 138S:S24-S30.

15 Hoitsma E, De Vries J, van Santen-Hoeufft M, et al. Impact of pain in a Dutch sarcoidosis patient population. Sarcoidosis Vasc Diffuse Lung Dis 2003; 20:33-9.

16 Aggarwal AN, Sahu KK, Gupta D. Fatigue and health-related quality of life in patients with pulmonary sarcoidosis treated by oral corticosteroids. Sarcoidosis Vasc Diffuse Lung Dis 2016; 33:124-9.

17 Drent M, Marcellis R, Lenssen A, De Vries J. Association between physical functions and quality of life in sarcoidosis. Sarcoidosis Vasc Diffuse Lung Dis 2014; 31:117-28.

18 Drent $\mathrm{M}$, Wirnsberger RM, Breteler $\mathrm{MH}$, et al. Quality of life and depressive symptoms in patients suffering from sarcoidosis. Sarcoidosis Vasc Diffuse Lung Dis 1998; 15:59-66.

19 Jastrzebski $D$, Ziora $D$, Lubecki $M$, et al. Fatigue in sarcoidosis and exercise tolerance, dyspnea, and quality of life. Adv Exp Med Biol 2015; 833:31-6.

20 Michielsen HJ, Drent M, Peros-Golubicic T, De Vries J. Fatigue is associated with quality of life in sarcoidosis patients. Chest 2006; 130:989-94.

21 Michielsen HJ, Peros-Golubicic T, Drent M, De Vries J. Relationship between symptoms and quality of life in a sarcoidosis population. Respiration 2007; 74:401-5.

22 Wirnsberger RM, de Vries J, Breteler $\mathrm{MH}$, et al. Evaluation of quality of life in sarcoidosis patients. Respir Med 1998; 92:750-6.

23 Zajicek JP, Scolding NJ, Foster O, et al. Central nervous system sarcoidosis--diagnosis and management. QJM 1999; 92:103-17.

24 Cox CE, Donohue JF, Brown CD, et al. Health-related quality of life of persons with sarcoidosis. Chest 2004; 125:997-1004.

25 Marcellis RG, Lenssen AF, de Vries J, Drent M. Reduced muscle strength, exercise intolerance and disabling symptoms in sarcoidosis. Curr Opin Pulm Med 2013; 19:524-30. 
26 Drent M, Lower EE, De Vries J. Sarcoidosis-associated fatigue. Eur Respir J 2012; 40:255-63.

27 Stern BJ, Royal W, 3rd, Gelfand JM, et al. Definition and consensus diagnostic criteria for neurosarcoidosis: from the neurosarcoidosis consortium consensus group. JAMA Neurol 2018;75:15461553

28 Sodhi M, Pearson K, White ES, Culver DA. Infliximab therapy rescues cyclophosphamide failure in severe central nervous system sarcoidosis. Respir Med 2009; 103:268-73.

29 Moravan M, Segal BM. Treatment of CNS sarcoidosis with infliximab and mycophenolate mofetil. Neurology 2009; 72:337-40.

30 Joubert B, Chapelon-Abric C, Biard L, et al. Association of prognostic factors and immunosuppressive treatment with long-term outcomes in neurosarcoidosis. JAMA Neurol 2017; 74:1336-1344.

31 Gelfand JM, Bradshaw MJ, Stern BJ, et al. Infliximab for the treatment of CNS sarcoidosis: a multiinstitutional series. Neurology 2017; 89:2092-2100.

32 Cohen Aubart F, Bouvry D, Galanaud D, et al. Long-term outcomes of refractory neurosarcoidosis treated with infliximab. J Neurol 2017; 264:891-897.

33 Bitoun S, Bouvry D, Borie R, et al. Treatment of neurosarcoidosis: a comparative study of methotrexate and mycophenolate mofetil. Neurology 2016; 87:2517-2521.

$34 \mathrm{Kim}$ HS, Lee S, Kim JH. Real-world evidence versus randomized controlled trial: clinical research based on electronic medical records. J Korean Med Sci 2018; 33:e213.

35 Maassen H. Als standaardonderzoek tekort schiet. In Medisch Contact. Edited by; 2019:14-17.

36 Blackmore D, Siddiqi ZA. Diagnostic criteria for small fiber neuropathy. J Clin Neuromuscul Dis 2017; 18:125-131.

37 Casanova-Molla J, Grau-Junyent JM, Morales M, Valls-Sole J. On the relationship between nociceptive evoked potentials and intraepidermal nerve fiber density in painful sensory polyneuropathies. Pain 2011; 152:410-8.

38 Chen ES, Moller DR. Etiologies of sarcoidosis. Clin Rev Allergy Immunol 2015; 49:6-18.

39 Rage M, Van Acker N, Knaapen MW, et al. Asymptomatic small fiber neuropathy in diabetes mellitus: investigations with intraepidermal nerve fiber density, quantitative sensory testing and laser-evoked potentials. J Neurol 2011; 258:1852-64.

40 Lauria G, Hsieh ST, Johansson O, et al. European federation of neurological societies/peripheral nerve society guideline on the use of skin biopsy in the diagnosis of small fiber neuropathy. Report of a joint task force of the European federation of neurological societies and the peripheral nerve society. Eur J Neurol 2010; 17:903-12, e44-9.

41 Truini A, Biasiotta A, Di Stefano G, et al. Does the epidermal nerve fibre density measured by skin biopsy in patients with peripheral neuropathies correlate with neuropathic pain? Pain 2014; 155:828-32.

42 Tavakoli M, Quattrini C, Abbott C, et al. Corneal confocal microscopy: a novel noninvasive test to diagnose and stratify the severity of human diabetic neuropathy. Diabetes Care 2010; 33:1792-7.

43 Tavakoli M, Marshall A, Pitceathly R, et al. Corneal confocal microscopy: a novel means to detect nerve fibre damage in idiopathic small fibre neuropathy. Exp Neurol 2010; 223:245-50.

44 Stettner M, Hinrichs L, Guthoff R, et al. Corneal confocal microscopy in chronic inflammatory demyelinating polyneuropathy. Ann Clin Transl Neurol 2016; 3:88-100.

45 Jiang MS, Yuan Y, Gu ZX, Zhuang SL. Corneal confocal microscopy for assessment of diabetic peripheral neuropathy: a meta-analysis. Br J Ophthalmol 2016; 100:9-14.

46 Bosse-Henck A, Koch R, Wirtz H, Hinz A. Fatigue and excessive daytime sleepiness in sarcoidosis: prevalence, predictors, and relationships between the two symptoms. Respiration 2017; 94:186-197.

47 Elfferich MD, Nelemans PJ, Ponds RW, et al. Everyday cognitive failure in sarcoidosis: the prevalence and the effect of anti-TNF-alpha treatment. Respiration 2010; 80:212-9.

48 Tavee JO, Karwa K, Ahmed Z, et al. Sarcoidosis-associated small fiber neuropathy in a large cohort: clinical aspects and response to IVIG and anti-TNF alpha treatment. Respir Med 2017; 126:135-138.

49 Cunha FQ, Poole S, Lorenzetti BB, Ferreira SH. The pivotal role of tumour necrosis factor alpha in the development of inflammatory hyperalgesia. Br J Pharmacol 1992; 107:660-4.

50 Ferreira SH, Lorenzetti BB, Bristow AF, Poole S. Interleukin-1 beta as a potent hyperalgesic agent antagonized by a tripeptide analogue. Nature 1988; 334:698-700.

51 Uceyler N, Kafke W, Riediger N, et al. Elevated proinflammatory cytokine expression in affected skin in small fiber neuropathy. Neurology 2010; 74:1806-13. 
52 Wijnen PA, Cremers JP, Nelemans PJ, et al. M. Association of the TNF-alpha G-308A polymorphism with TNF-inhibitor response in sarcoidosis. Eur Respir J 2014; 43:1730-9.

53 Brines M, Dunne AN, van Velzen M, et al. ARA 290, a nonerythropoietic peptide engineered from erythropoietin, improves metabolic control and neuropathic symptoms in patients with type 2 diabetes. Mol Med 2015; 20:658-66.

54 Brines M, Cerami A. Emerging biological roles for erythropoietin in the nervous system. Nat Rev Neurosci 2005; 6:484-94.

55 Agnello D, Bigini P, Villa $P$, et al. Erythropoietin exerts an anti-inflammatory effect on the CNS in a model of experimental autoimmune encephalomyelitis. Brain Res 2002; 952:128-34.

56 Heij L, Niesters M, Swartjes M, et al. Safety and efficacy of ARA 290 in sarcoidosis patients with symptoms of small fiber neuropathy: a randomized, double-blind pilot study. Mol Med 2012; 18:1430-6.

57 Dahan A, Dunne A, Swartjes M, et al. ARA 290 improves symptoms in patients with sarcoidosisassociated small nerve fiber loss and increases corneal nerve fiber density. Mol Med 2013; 19:334-45.

58 Culver DA, Dahan A, Bajorunas D, et al. Cibinetide improves corneal nerve fiber abundance in patients with sarcoidosis-associated small nerve fiber loss and neuropathic pain. Invest Ophthalmol Vis Sci 2017; 58:BIO52-BIO60.

59 de Greef BTA, Hoeijmakers JGJ, Geerts M, et al. Lacosamide in patients with Nav1.7 mutations-related small fibre neuropathy: a randomized controlled trial. Brain 2019; 142:263-275.

60 Aganov S. Study of the effect of the VR technology on recovery of the autonomic nervous system in volunteers affected by stress. clinicaltrials.gov; NCT03532152

61 Hendriks CMR, Saketkoo LA, Elfferich MDP, et al. Sarcoidosis and work participation: the need to develop a disease-specific core set for assessment of work ability. Lung 2019, Epub May.

62 Barth J, de Boer WE, Busse JW, et al. Inter-rater agreement in evaluation of disability: systematic review of reproducibility studies. BMJ 2017; 356:j14.

63 Saketkoo LA, Escorpizo R, Keen KJ, et al. International classification of functioning, disability and health core set construction in systemic sclerosis and other rheumatic diseases: a EUSTAR initiative. Rheumatology (Oxford) 2012; 51:2170-6. 
Part III 

Valorisatie 



\section{Inleiding}

Sarcoïdose is een zeldzame (geschatte incidentie 10-20 per 100.000 personen/jaar) inflammatoire multisysteem aandoening die gekenmerkt wordt door ontstekingsactiviteit met vorming van niet-necrotiserende granulomen (granulomateuze ontsteking). Deze granulomen kunnen overal in het lichaam voorkomen, waarbij de longen, lymfeklieren en ogen het meest betrokken zijn, en leiden tot functieverlies van de desbetreffende organen. Mede gezien de zeldzaamheid wordt het niet altijd tijdig herkend en blijft soms een zoektocht voor arts en patiënt.

Als sarcoïdose het zenuwstelsel aantast, te weten de hersenen, het ruggenmerg en/of de dikke zenuwvezels, spreekt men van neurosarcoïdose. Deze presentatie kan op zichzelf staan of met een of meerdere lokalisaties elders in het lichaam gepaard gaan. Neurosarcoïdose is een complexe, ernstige en zeldzame manifestatie van sarcoïdose. Geschat wordt dat zo'n 5-10\% van de sarcoïdosepopulatie een manifestatie heeft in het zenuwstelsel. De klinische uitingen zijn heterogeen, afhankelijk van de betrokken lokalisatie van het zenuwstelsel. Doordat deze manifestaties zo zeldzaam zijn, zijn onderzoeken naar neurosarcoïdose schaars. Deze manifestaties kunnen echter wel levensbedreigend zijn en leiden tot mortaliteit, maar vooral tot een hoge morbiditeit. Daarom is het van groot belang inzicht te krijgen in de verschillende presentaties van neurosarcoïdose ondanks het feit dat het om zeldzame manifestaties van de ziekte gaat. In dit proefschrift wordt het voorkomen van de verschillende manifestaties van neurosarcoïdose in een groot neurosarcoïdose cohort in Nederland beschreven (hoofdstuk 3). Rekening houden met het tegelijkertijd voorkomen van andere sarcoïdose manifestaties en de relatie met geslacht en ras zijn van belang om het bewustzijn aangaande het voorkomen van de verschillende neurosarcoïdosemanifestaties te vergroten.

Sarcoïdose kan leiden tot een heel scala aan orgaan-specifieke symptomen. Echter, sarcoïdosepatiënten ervaren veelal ook niet orgaan-specifieke symptomen, zoals pijn, vermoeidheid, cognitieve problemen, depressieve en angstklachten, slaapstoornissen en verminderde inspanningstolerantie. Het idee is dat dit niet zozeer wordt veroorzaakt door de granulomateuze ontsteking in de betrokken organen, maar meer door de vrijgekomen cytokines (algemenere ontstekingscellen) in de bloedbaan. Deze symptomen zijn moeilijk te objectiveren maar leiden wel tot een forse afname van de kwaliteit van leven in deze, toch vaak relatief jonge, patiëntenpopulatie (zie ook hoofdstuk 9). Medicamenteuze behandeling heeft relatief weinig effect op het verminderen van deze symptomen. In dit proefschrift hebben we de prevalentie van deze symptomen bij sarcoïdose patiënten binnen Europa in kaart gebracht 
(hoofdstuk 8) en specifiek bij neurosarcoïdose patiënten gekeken naar cognitieve problemen in vergelijking met algemene sarcoïdose patiënten (hoofdstuk 4).

Het lastige is dat patiënten niet altijd serieus genomen worden als het gaat om moeilijk te objectiveren niet-specifieke symptomen. $\mathrm{Er}$ zijn verschillende vragenlijsten ontwikkeld om deze niet-specifieke symptomen te meten: de Fatigue Assessment Scale (FAS) voor vermoeidheid, de Small Fiber Neuropathy Screening List (SFNSL) voor dunne vezelneuropathie geassocieerde symptomen en de Cognitive Failure Questionnaire (CFQ) voor cognitieve problemen (zie ook Appendix). Al deze symptomen blijken veel voor te komen. Cognitieve problemen komen naar schatting voor bij 35\%, dunne vezelneuropathie (DVN) gerelateerde klachten bij $40-60 \%$ en moeheid bij $50-80 \%$ van de algemene sarcoïdosepopulatie. Specifiek binnen de neurosarcoïdosepopulatie bleek dit een nog groter probleem (zie hoofdstuk 4).

$\mathrm{Er}$ is aangetoond dat DVN veelvuldig voorkomt bij sarcoïdosepatiënten. DVN kan leiden tot sensorische pijnklachten en autonome dysfunctie. Dit laatste kan gepaard gaan met klachten als hartkloppingen, duizeligheid, droge ogen of mond, urine-incontinentie, maagdarm-problemen en seksuele dysfunctie. DVN is moeilijk te diagnosticeren. Er zijn verschillende testen die allen geen $100 \%$ zekerheid geven of dit fenomeen aan- dan wel afwezig is. Het diagnostisch traject is dan ook regelmatig moeizaam. Om een eerste screening naar het mogelijk bestaan van DVN-gerelateerde symptomen in de praktijk makkelijker te maken is de reeds genoemde SFNSL ontwikkeld. Om beter inzicht te krijgen in het verloop in de tijd hebben we in dit proefschrift de 'minimal important difference' van deze lijst beschreven, dat is de minimale verandering in de score van de vragenlijst die voor de patiënt klinisch relevant is (hoofdstuk 7). Dit kan in vervolgstudies naar het effect van behandelingen op de symptomen van een patiënt gebruikt (gaan) worden.

\section{Maatschappelijke relevantie}

Sarcoïdose is een ziekte die vooral voorkomt bij jongvolwassenen (tussen de 25-45 jaar), zowel bij mannen als vrouwen, en een hogere incidentie kent in NoordEuropa en bij Afro-Amerikanen. Sarcoïdosepatiënten staan bij diagnose over het algemeen nog volop in het leven, waarbij ze vaak een combinatie van gezin en werk/carrière hebben. Orgaan-gerelateerde klachten alsmede niet-orgaan gerelateerde, niet specifieke klachten van de sarcoïdose kunnen derhalve grote gevolgen hebben voor het dagelijks functioneren en het sociale leven.

Zoals eerder genoemd is neurosarcoïdose een zeldzame en ernstige manifestatie van sarcoïdose. De meeste uitingen van neurosarcoïdose zijn erg invaliderend en leiden 
regelmatig tot restschade, ondanks immunosuppressieve behandeling. Daarom is het des te belangrijker om de bewustwording te vergroten bij behandelaren, zodat neurosarcoïdose tijdiger herkend en behandeld wordt om zo de restschade hopelijk te kunnen beperken.

Moeheid, cognitieve problemen (geheugen- en concentratiestoornissen) en pijn veroorzaken regelmatig beperkingen in het dagelijks functioneren vergeleken met gezonde leeftijdsgenoten. Dit leidt bij neurosarcoïdose tot nog meer problemen in hun arbeids- en sociale leven dan bij sarcoïdose in het algemeen. Dit veroorzaakt ook stress, frustratie en afname van kwaliteit van leven. Aangezien de patiënten er meestal goed uitzien, is de aandoening niet zichtbaar aan de buitenkant. Dit leidt vaak tot onbegrip, 'je ziet er toch goed uit?'. Het is derhalve van groot belang dat ook de niet-orgaan gerelateerde klachten herkend worden door de behandelend medisch specialisten en paramedici zodat patiënt gehoord wordt en er, indien mogelijk, met een adequaat behandelplan gestart kan worden. De bestaande vragenlijsten voor niet-orgaan gerelateerde klachten (o.a. FAS, SFNSL, CFQ) kunnen hieraan bijdragen. Hierbij is de manier van beoordeling van de uitkomst van de vragenlijsten van belang, oftewel een voor de patiënt klinisch relevant verschil in de score op de vragenlijst in de loop van de tijd. Voor de FAS was dit reeds bekend maar voor de SFNSL nog niet.

Onze onderzoeksresultaten hebben bijgedragen tot het vergroten van de kennis over neurosarcoïdose. Hopelijk hebben de resultaten een aanzet gegeven tot het vergroten van de bewustwording over deze zeldzame manifestatie van sarcoïdose, zowel bij longartsen als neurologen. Daarnaast is het relevant dat we nu hebben aangetoond dat de niet-orgaan gerelateerde, niet-specifieke klachten ook in andere landen in Europa in vergelijkbare mate voorkomen.

Het gebruik van de SFNSL ter objectivering van DVN-gerelateerde klachten is eens te meer gestimuleerd aangezien er nu ook bekend is wat een klinisch relevante verandering voor de patiënt is. Dit kan nu gebruikt gaan worden in zowel de klinische praktijk alsmede in wetenschappelijk studies naar de behandeling van DVN om beter te kunnen beoordelen of een behandeling effectief is voor de patiënt (i.t.t. alleen statistisch significant verschillen).

\section{Doelgroepen}

Al onze studies en studieresultaten zijn uitgevoerd met behulp van sarcoïdosepatiënten inclusief neurosarcoïdose patiënten, onze doelgroep. Het betreft klinisch onderzoek wat erop gericht is meer inzicht en begrip te creëren voor deze zeldzame ziekte. Het 
streven is zo verbetering in de zorgverlening en daarmee tot verbeteringen in het welzijn van de patiënten te creëren. Sarcoïdose is een multisysteem aandoening die, afhankelijk van de aangedane organen, erg invaliderend kan zijn en afname van kwaliteit van leven kan veroorzaken. Patiënten voelen zich vaak niet gehoord of serieus genomen met name als het gaat om niet-orgaan gerelateerde symptomen. Deze symptomen komen veelvuldig voor, zeker bij patiënten met neurosarcoïdose. Het streven is middels meer inzicht in het voorkomen van deze symptomen en kennis over hoe deze symptomen te kunnen objectiveren meer begrip en inzicht te creëren bij medisch specialisten.

De onderzoeksresultaten in dit proefschrift beogen medisch specialisten en andere betrokken zorgverleners meer bewust te maken van het bestaan van niet-orgaan gebonden klachten zoals cognitieve problemen, moeheid en DVN-gerelateerde klachten, maar ook het voorkomen van verschillende neurosarcoïdose manifestaties. Daarnaast zal het de kennis over het bestaan en de bruikbaarheid van vragenlijsten om te proberen de niet-specifieke symptomen te objectiveren kunnen verruimen.

Aangezien praktisch alle organen betrokken kunnen zijn bij het ziekteproces is een multidisciplinaire benadering van sarcoïdosepatiënten noodzakelijk. Dit maakt dat verschillende medisch specialisten betrokken zijn en specifieke kennis over de vele klachten en manifestaties die patiënten kunnen hebben vereist is. Daarnaast is het van belang dat medisch specialisten op de hoogte zijn van risicofactoren op het ontwikkelen van deze klachten. Dit onderzoek draagt daaraan bij.

\section{ACTIVITEITEN EN PRODUCTEN}

\section{Kennisdeling}

Het voordeel van klinisch onderzoek is dat relevante bevindingen van directe waarde zijn voor de (neuro)sarcoïdosepatiënten en hun behandelaren. Dit proefschrift bevat dan ook uitsluitend klinisch (toegepast) wetenschappelijk onderzoek. We streven erna om de onderzoeksresultaten zodanig te implementeren in de zorg dat de patiënt zich beter gehoord en begrepen voelt. Door het standaard toepassen van de in het proefschrift genoemde vragenlijsten en de toegenomen bewustwording bij behandelaren voor zeldzamere sarcoïdose manifestaties (m.n. neurosarcoïdose manifestaties) en het voorkomen van de niet-specifieke symptomen, zal de zorg verbeteren. Een voorwaarde voor het implementeren van de onderzoeksresultaten is dat zowel behandelaren als sarcoïdosepatiënten op de hoogte zijn van de nieuwste ontwikkelingen en wetenschappelijke inzichten. Aangezien de begeleiding van 
sarcoïdosepatiënten een multidisciplinair karakter heeft, is het de uitdaging om zoveel mogelijk disciplines te bereiken.

De onderzoeken beschreven in dit proefschrift zijn gepubliceerd in internationale tijdschriften, dit betreft zowel sarcoïdose, long als neurologische tijdschriften. Tevens hebben de onderzoeksresultaten geleid tot wetenschappelijke voordrachten op zowel nationale (masterclass sarcoïdose) als internationale (World Association of Sarcoidosis and other Granulomatous Disorders [WASOG] en American Thoracic Society [ATS]) congressen. Tijdens patiëntenbijeenkomsten en refereerbijeenkomsten is getracht de kennis uit dit proefschrift te delen met collega's en patiënten. Mede hierdoor zijn de resultaten beschikbaar gekomen voor behandelaren betrokken bij de zorg van sarcoïdosepatiënten.

\section{Patient empowerment}

De patiënt anno nu, en zeker de sarcoïdosepatiënt, is over het algemeen een patiënt die actief betrokken wil zijn bij zijn/haar behandeling en op de hoogte wil blijven van de nieuwste medische ontwikkelingen. Het actief participeren in het vervolgen van de eigen ziektelast d.m.v. vragenlijsten is daar een voorbeeld van.

Een goed geïnformeerde patiënt is beter gemotiveerd om een eigen rol in te nemen binnen zijn/haar eigen ziekteproces. Tijdens lezingen voor de leden van de Sarcoïdose Belangenvereniging Nederland (Sarcoidose.nl) hebben we middels informatie en kennisoverdracht dit nagestreefd. Tijdens deze bijeenkomsten werd de diversiteit van sarcoïdose en de uiteenlopende symptomen en presentatie van klachten besproken. $\mathrm{Na}$ afloop werden de patiënten en hun partners in de gelegenheid gesteld vragen te stellen. Ook bleek uit ons onderzoek dat het betrekken van partners van belang is bij de behandeling van sarcoïdose patiënten.

\section{Innovatie}

Op het moment van starten van deze thesis was de kennis over manifestaties van neurosarcoïdose beperkt. Onderzoek beschreven in dit proefschrift maakt gebruik van een groot nationaal Nederlands cohort van neurosarcoïdosepatiënten wat geleid heeft tot meer inzicht in deze ernstige, complexe en zeer zeldzame ziekte.

Daarnaast verschaffen de beschreven onderzoeken inzicht in de impact van niet-orgaan gerelateerde symptomen die veelvuldig voorkomen bij sarcoïdose. Niet alleen in Nederland, maar nu ook in andere landen in Europa rapporteerden sarcoïdosepatiënten deze symptomen met een lagere kwaliteit van leven als gevolg. Verbetering 
van het inzicht hierin draagt bij tot meer bewustwording bij de behandelend medisch specialisten maar ook bij bijvoorbeeld UWV- en ARBO-artsen die deze patiënten begeleiden bij problemen rondom het werk. Patiënten voelen zich vaak niet gehoord en niet begrepen. Hopelijk leiden onze studies tot meer begrip bij medisch specialisten en andere zorgverleners en een betere zorg voor deze patiëntenpopulatie.

De gebruikte vragenlijsten zijn makkelijk te implementeren in de dagelijkse zorg van deze patiënten. Het gebruik hiervan zal tevens leiden tot meer inzicht en betere zorg op maat. Dé sarcoïdosepatiënt bestaat niet, iedereen is uniek en behoeft een behandelplan afgestemd op de persoonlijke omstandigheden. Aangezien veel nietspecifieke symptomen niet responderen op de gebruikelijke behandeling is het des te belangrijker dat patiënten zich gehoord en begrepen voelen.

\section{Toekomstvisie}

Vanwege de zeldzaamheid is de zorg voor patiënten met sarcoïdose extra uitdagend. Zowel patiënten, artsen als onderzoekers lopen tegen knelpunten aan zoals het moeizaam stellen van een diagnose, het vinden van zorgverleners met voldoende kennis en kunde en problemen met beschikbaarheid of vergoeding van eventuele medicamenteuze therapie. Door professionalisering van de patiëntenvereniging, het centraliseren van zorg in expertise- en behandelcentra, het zichtbaar en bereikbaar maken van optimale zorg en kennis voor alle patiënten en zorgverleners in Nederland, en uiteraard internationale samenwerking zal in de toekomst de zorg voor patiënten met sarcoïdose verder kunnen verbeteren.

Ook is het van belang om materiaal van sarcoïdosepatiënten te verzamelen in een biobank om zo onderzoek in grotere populatie met een zeldzame ziekte naar de genetische achtergrond van sarcoïdose mogelijk te maken.

Voor onderzoekers is het bestuderen van sarcoïdose ook een extra uitdaging. Om bepaalde eigenschappen of kenmerken van patiënten met sarcoïdose goed in kaart te brengen zijn over het algemeen grote groepen patiënten nodig. Bij studies naar zeldzame manifestaties van sarcoïdose, zoals neurosarcoïdose, wordt er bij voorkeur samengewerkt met meerdere ziekenhuizen, waarvoor een laagdrempelige uitwisseling van medische gegevens een voorwaarde is en uitgebreidere logistiek noodzakelijk. Ook het ontwikkelen van nieuwe medicijnen voor kleine patiëntengroepen is moeilijk. Het is voor grote farmaceutische bedrijven namelijk aantrekkelijker om medicijnen te ontwikkelen voor ziekten die veel vaker voorkomen. 
De uitkomsten van de onderzoeken beschreven in dit proefschrift hebben geleid tot meer kennis over de problemen bij (neuro)sarcoïdosepatiënten. Het is belangrijk dat zorgverleners kennisnemen van de resultaten en beseffen dat lang niet altijd de problemen van chronische zieke patiënten, zoals sarcoïdosepatiënten, vast te leggen zijn met objectieve medische testen. Dit is van belang voor de zorgverleners die sarcoïdosepatiënten begeleiden, maar vooral ook ARBO- en keuringsartsen die de belastbaarheid moeten vaststellen om te beoordelen of een patiënt instaat is om werkzaamheden uit te voeren of in mindere mate of niet. Hier geldt bij uitstek dat het ontbreken van bijvoorbeeld afwijkende longfunctiewaarden niet betekent dat er 'dus niets aan de hand is'.

Het is noodzakelijk dat er aandacht geschonken wordt aan de niet-specifieke, nietorgaan gerelateerde symptomen bij sarcoïdose en dat hier ook aandacht voor is in de nationale en internationale richtlijnen. Hiervoor dienen onze aanbevelingen gesteund te worden door diverse organisaties, onder andere de Nederlandse Vereniging van Artsen voor Longziekten en Tuberculose (NVALT) en de World Association of Sarcoidosis and other Granulomatous Disorders (WASOG), maar ook organisaties als het UWV. Inmiddels heeft een vervolgonderzoek plaatsgevonden waarin de problematiek die sarcoïdose-patiënten zelf ervaren met keuringsinstanties in kaart is gebracht met als doel beter begrip te creëren voor deze moeilijke problematiek. Sarcoïdose is een grillige ziekte die zich bij ieder mens anders presenteert. Bij de beoordeling van individuen, zoals tijdens een arbeidsongeschiktheidskeuring, dient de aard en ernst van de aandoening, en vooral de gevolgen daarvan voor die specifieke patiënt meegewogen te worden in het advies. Naast ziekte gerelateerde klachten en functionele stoornissen beïnvloeden vermoeidheid, concentratievermogen, geheugen, conditie- en energieverlies of iemand al dan niet in staat is tot het verrichten van arbeid en in welke mate. ledere patiënt is uniek en behoeft een eigen benadering. Dit geldt overigens voor iedereen met een chronische aandoening, zeker niet alleen voor sarcoïdosepatiënten. Helaas moeten we constateren dat uitermate reële klachten van de patiënt vaak vage klachten zijn voor de zorgverleners. Hier dient meer begrip voor te komen en de patiënt dient het gevoel te hebben serieus te worden genomen en een partner in het management van de ziekte te zijn.

Dé behandeling van sarcoïdose bestaat vooralsnog niet. Zoals reeds meerdere keren aangegeven is iedereen uniek en wat bij de een werkt, werkt bij de ander soms niet. Toekomstig onderzoek dient zich te richten op verdere studies naar de meest effectieve behandeling van (neuro)sarcoïdose, waarin een onderscheid in de verschillende manifestaties gemaakt dient te worden alsmede onderzoek naar behandeling van de 
niet-orgaan gerelateerde symptomen (moeheid, cognitieve problemen en DVNgerelateerde klachten).

Voor de verdere implementatie van de uitkomsten van de studies in dit proefschrift in de dagelijkse begeleiding van deze patiënten is het van belang de toenemende ICTmogelijkheden optimaal te benutten. Het zou geïntegreerd kunnen worden in de elektronische patiëntendossiers (EPD's) van de ziekenhuizen en wellicht kan er een app ontwikkeld worden, zodat patiënten op hun smartphone vragenlijsten kunnen invullen en scores kunnen vervolgen. Door toegang tot alle persoonlijke medische gegevens kunnen patiënten nog meer betrokken worden bij de regie van hun eigen ziektemanagement. 
Samenvatting 



\section{Inleiding}

Sarcoïdose is een inflammatoire multisysteem aandoening, gekenmerkt door de vorming van niet-necrotiserende granulomen in verschillende organen. ${ }^{1,2}$. leder orgaan kan bij het ziekteproces betrokken zijn, waarvan de lymfeklieren en longen het vaakst zijn aangedaan. Betrokkenheid van het zenuwstelsel, oftewel neurosarcoïdose, wordt gezien in ongeveer $5 \%$ van de patiënten. ${ }^{3-5}$ In autopsie studies wordt een prevalentie tot $25-50 \%$ beschreven. ${ }^{6,7}$ De oorzaak van de ziekte is tot op heden nog steeds niet geheel opgehelderd, maar lijkt een combinatie te zijn van een genetische predispositie leidend tot een uit de hand gelopen immuunrespons als reactie op omgevingsfactoren (infectieuze triggers, metaaldeeltjes, silica, of bestrijdingsmiddelen) of lichaamsvreemde stoffen (zoals inkt in tatoeages, en siliconen bevattende protheses).

Sarcoïdose komt overal ter wereld voor, zowel bij mannen als vrouwen als in alle rassen, met een predispositie voor relatief jongere mensen (24-45 jaar). ${ }^{1}$ De epidemiologie van sarcoïdose wordt wel beïnvloed door ras en geslacht. De incidentie van sarcoïdose is hoger bij negrö̈de mensen, in vergelijking met blanken. Bovendien presenteren zij zich vaker met ernstigere orgaanbetrokkenheid leidend tot een hogere mortaliteit en slechtere prognose. ${ }^{8-10}$ De prevalentie van de verschillende sarcoïdosemanifestaties verschilt per ras en geslacht. Oculaire, hepatogene en beenmergbetrokkenheid alsmede ernstigere pulmonale sarcoïdose komen vaker voor bij negroïde dan blanke sarcoïdosepatiënten, terwijl hypercalciëmie meer voorkomt bij blanken. ${ }^{3}$ Hypercalciëmie, pulmonale en cardiale sarcoïdose komen meer voor bij mannen en erythema nodosum, oculaire en hepatogene sarcoïdose meer bij vrouwen. $^{3,11}$

De klinische presentatie, het natuurlijk beloop en de prognose zijn erg variabel en onvoorspelbaar. ${ }^{2}$ Afhankelijk van de aangedane orgaansystemen en de ernst van granulomateuze inflammatie kunnen sarcoïdosepatiënten zich met verschillende symptomen presenteren. Naast orgaan-specifieke symptomen hebben sarcoïdosepatiënten frequent last van invaliderende niet-specifieke, niet orgaan-gerelateerde symptomen, ${ }^{12-14}$ zoals vermoeidheid, depressieve symptomen, pijn, angst en cognitieve problemen (geheugen- en concentratiestoornissen) leidend tot afname van kwaliteit van leven (KvL). ${ }^{14-21}$

Dit proefschrift is opgedeeld in twee delen:

De studies die in het eerste deel van dit proefschrift beschreven worden hebben als doel de prevalentie van de verschillende neurosarcoïdose manifestaties te beschrijven en de invloed van geslacht en ras hierop. Daarnaast was het doel om het cognitief functioneren in deze patiëntenpopulatie in kaart te brengen. 
De studies die in het tweede deel beschreven worden hebben als doel om de niet orgaan-gerelateerde, niet-specifieke symptomen verder in kaart te brengen (dunne vezelneuropathie geassocieerde symptomen, moeheid, cognitief falen, depressieve symptomen, angst) en de invloed op de kwaliteit van leven van zowel sarcoïdosepatiënten als hun partners.

\section{Overzicht bevindingen}

Hoofdstuk 1 geeft een algemene inleiding en overzicht van de epidemiologie en pathogenese van sarcoïdose en de klinische presentatie van neurosarcoïdose en de niet-specifieke klachten bij sarcoïdose. Daarnaast wordt er een samenvatting gegeven van de behandelopties van neurosarcoïdose en van de niet-specifieke symptomen vermoeidheid en dunne vezelneuropathie geassocieerde symptomen.

Hoofdstuk 2 bevat een overzicht van de huidige literatuur met betrekking tot neurosarcoïdose. De klinische presentatie is heterogeen, de meeste patiënten presenteren zich met hersenzenuwuitval, hoofdpijn of sensorische uitval. De diagnose neurosarcoïdose wordt gesteld o.b.v. classificatie criteria. In deze criteria is histopathologisch bewijs van niet-necrotiserende granulomen buiten het zenuwstelsel van cruciaal belang. Radiologische afwijkingen bij neuro-imaging zijn aspecifiek. Er zijn helaas geen biomarkers beschikbaar die specifiek genoeg zijn om sarcoïdose vast te stellen. Ondanks het starten van immunosuppressieve behandeling blijft nog steeds één derde van de neurosarcoïdose patiënten stabiel of vertonen progressie van de ziekte. Het diagnosticeren en behandelen van neurosarcoïdose patiënten blijft een uitdaging. Lange termijn studies zijn noodzakelijk om de sensitiviteit en specificiteit van de verschillende diagnostische onderzoeken alsmede de diagnostische criteria te evalueren. Daarnaast is het noodzakelijk om studies te verrichten om de prognose en de optimale behandelstrategie te bepalen.

Hoofstuk 3 geeft de resultaten weer van het onderzoek naar neurosarcoïdose manifestaties met de invloed van ras en geslacht en de behandelstrategieën in een Nederlandse neurosarcoïdose populatie. In een multicenter observationele studie werden 194 neurosarcoïdose patiënten geïncludeerd van twee tertiaire verwijscentra, het ILD Expertisecentrum in het St. Antonius Ziekenhuis Nieuwegein en Amsterdam Universitair Medisch Centrum in Nederland alsmede deelnemers aan het Neurosarcoïdose register in Nederland. De meest voorkomende neurosarcoïdose manifestaties zijn chronische meningitis (41\%), hersenzenuw dysfunctie (39\%), 
myelopathie (28\%) en intracerebrale pathologie (26\%). Leeftijd, geslacht en ras hadden geen invloed op de verschillende neurosarcoïdose manifestaties behalve perifere neuropathie. Deze komt meer voor bij mannen dan bij vrouwen (21\% versus $10 \%$ ). De meeste neurosarcoïdose patiënten werden behandeld met prednison (89\%), methotrexaat (46\%) of infliximab (21\%). Totaal kregen 102 patiënten $(53 \%) 2^{\mathrm{e}}$ of $3^{\mathrm{e}}$ lijnsbehandeling. Meer dan de helft van de patiënten werden met meerdere immunosuppressiva tegelijkertijd behandeld.

Hoofdstuk 4 beschrijft de resultaten van de studie naar cognitief falen van neurosarcoïdose patiënten, waarbij een vergelijking gemaakt wordt met een algemene sarcoïdose populatie. Cognitief falen is geassocieerd met geheugen- en concentratieproblemen. Om cognitief falen in de kaart te brengen hebben we gebruik gemaakt van de Cognitive Failure Questionnaire (CFQ). Een cross-sectioneel webgebaseerd onderzoek werd verricht onder 131 Nederlandse neurosarcoïdose patiënten. De gemiddelde CFQ-score was significant hoger in de neurosarcoïdose populatie $(45.6 \pm 20.7)$ dan in de algemene sarcoïdose populatie $(36.2 \pm 15.9 ; p<0.0001)$. Hoge CFQ-scores ( $\geq 43$ ) werden gevonden bij $55.7 \%$ van de neurosarcoïdosepatiënten t.o.v. 33.9\% van de algemene sarcoïdose populatie $(p<0.0001)$. De Fatigue Assessment Scale (FAS) score (OR 21.4) en de Small Fiber Neuropathy Screenings List (SFNSL) score (OR 4.3) zijn de sterkste positieve voorspellers voor een hoge CFQ-score. Cognitief falen is een significant probleem bij neurosarcoïdose patiënten.

Hoofdstuk 5 geeft een overzicht van de huidige literatuur met betrekking tot de diagnostiek en behandeling van neurosarcoïdose, inclusief dunne vezelneuropathie (DVN). De klinische manifestatie en het beloop van neurosarcoïdose zijn variabel. Hoewel neurosarcoïdose optreedt bij ongeveer $10 \%$ van de sarcoïdose patiënten, kan dit leiden tot aanzienlijke morbiditeit en zelfs soms mortaliteit. Recente richtlijnen hebben de criteria voor de diagnose neurosarcoïdose verder verduidelijkt. Er zijn echter nog geen duidelijke richtlijnen over bij wie, wanneer en hoe behandeling te starten. Het starten van behandeling hangt af van de presentatie, uitgebreidheid en ernst van de neurosarcoïdose. Qua evidence-based behandeling zijn er slechts enkele gerandomiseerde, gecontroleerde studies verricht. Vandaar dat er, in de huidige literatuur, aspecten ten aanzien van de behandeling van (neuro)sarcoïdose nog niet duidelijk uitgezocht zijn. Een multidisciplinaire benadering in de behandeling van sarcoïdose wordt sterk aanbevolen.

Hoofdstuk 6 geeft een overzicht van de huidige literatuur over DVN. DVN veroorzaakt een hoge morbiditeit vanwege invaliderende symptomen die de KvL beïnvloeden. 
Patiënten kunnen baat hebben bij het diagnosticeren van DVN, zelfs als er geen specifieke oorzaak gevonden kan worden en er nog geen specifieke behandeling beschikbaar is. Er zijn diagnostische criteria voorgesteld, echter is er geen gouden standaard en elke diagnostische test heeft zijn beperkingen. De diagnose DVN vereist een combinatie van typische symptomen, abnormale bevindingen bij de neurologische test(en) en de afwezigheid van dikke vezel betrokkenheid. Clinici dienen op de hoogte te zijn van overlappende symptomen zijn tussen DVN en fibromyalgie. Behandeling van DVN is vaak lastig, zelfs wanneer de onderliggende oorzaak geïdentificeerd is en deze adequaat behandeld wordt. Helaas is vaak alleen symptomatische behandeling mogelijk middels neuropathische pijnstilling. Bewustzijn van DVN en de hieraan gerelateerde symptomen is van groot belang. Richtlijnen voor de juiste diagnostische work-up met behulp van een stapsgewijze benadering met een combinatie van diagnostische testen zijn noodzakelijk.

Hoofdstuk 7 geeft de resultaten weer van het onderzoek naar de minimal important difference (MID) van de SFNSL. De MID is de kleinste verandering in een score op een vragenlijst die klinisch relevant is voor de patiënt. Eerdere studies hebben aangetoond dat $40-60 \%$ van de patiënten met sarcoïdose aan DVN geassocieerde symptomen lijdt, wat de KvL aanzienlijk beïnvloedt. Het nut van een vragenlijst in de klinische praktijk en wetenschappelijk onderzoek is mede afhankelijk van de interpreteerbaarheid. Ter bepaling van deze interpreteerbaarheid is het noodzakelijk om de smallest detectable change (SDC) en de minimal important difference (MID) te weten. Patiënten met neurosarcoïdose en/of sarcoïdose geassocieerde DVN-symptomen ( $n=138)$ die aangemeld waren in het online Nederlandse neurosarcoïdose register hebben geparticipeerd in een prospectieve, longitudinale studie. De SFNSL werd zowel aan het begin als na 6 maanden follow-up door 89 van de 138 patiënten voltooid. De MID van de SFNSL is 3.5 punt voor een klinisch relevante verandering in 6 maanden tijd. De MID kan gebruikt worden in de follow-up en behandeling van DVN-geassocieerde symptomen bij patiënten met sarcoïdose, hoewel met enige voorzichtigheid, aangezien de SDC hoger bleek dan de MID.

Hoofdstuk 8 beschrijft de de ziektelast vanuit Europees patiënten perspectief die veroorzaakt wordt door sarcoïdose-geassocieerde symptomen. De klinische manifestaties van sarcoïdose variëren sterk, afhankelijk van de intensiteit van de inflammatie en aangedane organen. Een cross-sectioneel, webgebaseerd onderzoek naar klachten (zowel orgaan-gerelateerd en niet-specifiek, niet-orgaangerelateerd) werd uitgevoerd onder sarcoïdose patiënten in Denemarken, Duitsland en Nederland. De survey werd ingevuld door 1072 sarcoïdose patiënten (152 Denen, 532 Duitsers en 
388 Nederlanders). Bijna alle patiënten rapporteerden sarcoïdose-geassocieerde klachten (zowel orgaan-gerelateerd en niet-specifiek, niet-orgaan-gerelateerd). Moeheid wordt gemeld door vrijwel alle patiënten (90\%), gevolgd door DVNgeassocieerde (86\%) en pulmonale symptomen (72.4\%). Meer dan 50\% van de patiënten werd behandeld met prednison, wat vergelijkbaar was in alle drie de landen. De $2^{\mathrm{e}}$ en $3^{\mathrm{e}}$ lijns behandeling verschilt daarentegen significant tussen Denemarken, Duitsland en Nederland. Sarcoïdose patiënten in Denemarken, Duitsland en Nederland presenteren zich met vergelijkbare orgaan-gerelateerde en niet-specifieke, niet-orgaan gerelateerde klachten.

Hoofdstuk 9 beschrijft een onderzoek naar de KvL bij zowel sarcoïdose patiënten als hun partners. De gevolgen van sarcoïdose zijn uitgebreid en de symptoombelasting heeft grote invloed op de KvL van patiënten. De KvL van paren die leven met sarcoïdose is echter nog niet onderzocht. Sarcoïdose patiënten, bekend in het Maastrichts Universitair Medisch Centrum ( $n=443)$ en hun partners $(n=208)$ werden geïncludeerd in de studie. Zij hebben meerdere vragenlijsten ingevuld, waaronder de World Health Organization Quality of Life - BREF (WHOQOL-BREF), FAS, SFNSL en CFQ. De KvL van de partners en van sarcoïdose patiënten was verminderd in vergelijking met de gezonde controles (partners in mindere mate dan patiënten), vooral met betrekking tot het domein lichamelijke gezondheid. Alle bestudeerde niet-specifieke symptomen, evenals sociale steun, voorspelde één of meer $K v L$ domeinen van de sarcoïdose patiënten, maar deze factoren voorspelden de KvL van de partners niet. Bij de behandeling van sarcoïdose is het belangrijk om niet alleen de patiënten centraal centraal te stellen, maar ook hun partners daarbij te betrekken.

Hoofdstuk 10 bevat de Engelstalige samenvatting met daarin de belangrijkste bevindingen van de onderzoeken beschreven in dit proefschrift, een algemene discussie en de hieruit voortkomende mogelijkheden voor toekomstig onderzoek. Dit proefschrift beschrijft de prevalentie en de ziektelast van neurosarcoïdose en de niet-specifieke symptomen, waaronder DVN. Ondanks dat de ziektelast hoog blijkt te zijn onder sarcoïdose patiënten, met name veroorzaakt door niet-specifieke symptomen, zoals DVN geassocieerde symptomen, moeheid, en cognitieve problemen, bestaan tot op heden geen richtlijnen voor de wijze van het in kaart brengen van deze beperkingen bij sarcoïdose. Ook de behandeling van zowel neurosarcoïdose als DVN zijn lastig mede door het ontbreken van goede richtlijnen. In dit proefschrift is getracht een begin te maken met de ontwikkeling van een richtlijn voor de diagnostiek en behandeling van neurosarcoïdose. Door middel van de bepaling van de minimaal relevante verandering (MID, minimal important difference) op de DVN-vragenlijst heeft dit proefschrift 
bijgedragen aan de interpreteerbaarheid van het effect van behandeling op de klachten van de patiënten.

Voor patiënten is het cruciaal dat de voor de dokter vaak vage klachten serieus worden genomen aangezien deze een enorme invloed op de $\mathrm{KvL}$ hebben. Toekomstig wetenschappelijk is onderzoek noodzakelijk om te bepalen welke diagnostische modaliteiten en behandeling het meest optimaal is voor de verschillende neurosarcoïdose manifestaties. Daarnaast dient een adequaat diagnostisch stappenplan te worden ontwikkeld voor de diagnostiek van DVN, alsmede adequate behandelingsopties. Ook al heeft sarcoïdose en dunne vezelneuropathie een enorme impact, zijn er helaas tot op heden geen goede behandelopties voorhanden. Ter bevordering van uitbreiding van de behandelopties is internationale samenwerking, inclusief samenwerking met patiëntenorganisaties van groot belang. Altijd dient hierbij het belang en daarmee verbetering van de KvL en verminderen van de ziektelast van de patiënt voorop te staan. 


\section{Referenties}

1. Valeyre D, Prasse A, Nunes H, et al. Sarcoidosis. Lancet. 2014;383(9923):1155-67.

2. Statement on sarcoidosis. Joint Statement of the American Thoracic Society (ATS), the European Respiratory Society (ERS) and the World Association of Sarcoidosis and Other Granulomatous Disorders (WASOG) adopted by the ATS Board of Directors and by the ERS Executive Committee, February 1999. Am J Respir Crit Care Med. 1999;160(2):736-55.

3. Judson MA, Boan AD, Lackland DT. The clinical course of sarcoidosis: presentation, diagnosis, and treatment in a large white and black cohort in the United States. Sarcoidosis Vasc Diffuse Lung Dis. 2012;29(2):119-27.

4. Fritz D, van de Beek D, Brouwer MC. Clinical features, treatment and outcome in neurosarcoidosis: systematic review and meta-analysis. BMC Neurol. 2016;16(1):220.

5. Ungprasert $\mathrm{P}$, Crowson CS, Matteson EL. Characteristics and long-term outcome of neurosarcoidosis: a population-based study from 1976-2013. Neuroepidemiology. 2017;48(3-4):87-94.

6. Joseph FG, Scolding NJ. Sarcoidosis of the nervous system. Pract Neurol. 2007;7(4):234-44.

7. Iannuzzi MC, Rybicki BA, Teirstein AS. Sarcoidosis. N Engl J Med. 2007;357(21):2153-65.

8. Mirsaeidi M, Machado RF, Schraufnagel D, et al. Racial difference in sarcoidosis mortality in the United States. Chest. 2015;147(2):438-49.

9. Kirkil G, Lower EE, Baughman RP. Predictors of mortality in pulmonary sarcoidosis. Chest. 2018;153(1):105-13.

10. Gerke AK, Judson MA, Cozier YC, et al. Disease burden and variability in sarcoidosis. Ann Am Thorac Soc. 2017;14(Supplement_6):S421-S8.

11. Ungprasert P, Crowson CS, Matteson EL. Influence of gender on epidemiology and clinical manifestations of sarcoidosis: a population-based retrospective cohort study 1976-2013. Lung. 2017;195(1):87-91.

12. Hoitsma E, De Vries J, van Santen-Hoeufft M, et al. Impact of pain in a Dutch sarcoidosis patient population. Sarcoidosis Vasc Diffuse Lung Dis. 2003;20(1):33-9.

13. Hendriks C, Drent M, De Kleijn W, et al. Everyday cognitive failure and depressive symptoms predict fatigue in sarcoidosis: a prospective follow-up study. Respir Med. 2018;138S:S24-S30.

14. Drent M, Strookappe B, Hoitsma E, De Vries J. Consequences of sarcoidosis. Clin Chest Med. 2015;36(4):727-37.

15. Wirnsberger RM, de Vries J, Breteler $\mathrm{MH}$, et al. Evaluation of quality of life in sarcoidosis patients. Respir Med. 1998;92(5):750-6.

16. Michielsen HJ, Peros-Golubicic T, Drent M, De Vries J. Relationship between symptoms and quality of life in a sarcoidosis population. Respiration. 2007;74(4):401-5.

17. Michielsen HJ, Drent M, Peros-Golubicic T, De Vries J. Fatigue is associated with quality of life in sarcoidosis patients. Chest. 2006;130(4):989-94.

18. Jastrzebski $\mathrm{D}$, Ziora $\mathrm{D}$, Lubecki $\mathrm{M}$, et al. Fatigue in sarcoidosis and exercise tolerance, dyspnea, and quality of life. Adv Exp Med Biol. 2015;833:31-6.

19. Drent $\mathrm{M}$, Wirnsberger $\mathrm{RM}$, Breteler $\mathrm{MH}$, et al. Quality of life and depressive symptoms in patients suffering from sarcoidosis. Sarcoidosis Vasc Diffuse Lung Dis. 1998;15(1):59-66.

20. Drent M, Marcellis R, Lenssen A, De Vries J. Association between physical functions and quality of life in sarcoidosis. Sarcoidosis Vasc Diffuse Lung Dis. 2014;31(2):117-28.

21. Aggarwal AN, Sahu KK, Gupta D. Fatigue and health-related quality of life in patients with pulmonary sarcoidosis treated by oral corticosteroids. Sarcoidosis Vasc Diffuse Lung Dis. 2016;33(2):124-9. 

List of publications 



\section{List of scientific publications and presentations}

Voortman M, Hendriks CMR, Lodder P, Drent M, De Vries J. Quality of life of couples living with sarcoidosis.

Respiration 2019; in press.

Voortman M, Fritz D, Vogels OJM, van de Beek D, De Vries J, Brouwer MC, Drent M. Clinical manifestations of neurosarcoidosis in the Netherlands.

J Neurol Neurosci 2019;10(2):292. doi: 10.21767/2171-6625.1000292.

Voortman M, Hendriks CMR, Elfferich MDP, Bonella F, Møller J, De Vries J, Costabel U, Drent $M$. The burden of sarcoidosis symptoms from a patients' perspective.

Lung 2019;197(2):155-161. doi: 10.1007/s00408-019-00206-7. PMID: 30778661.

Voortman M, Drent M, Baughman RB. Management of neurosarcoidosis: a clinical challenge.

Curr Opin Neurol 2019;32(3):475-483. doi: 10.1097/WCO.0000000000000684. Review. PMID: 30865007.

Voortman M, De Vries J, Hendriks CMR, Elfferich MDP, Wijnen PAHM, Drent M. Risk of everyday cognitive failure in patients with neurosarcoidosis.

Sarcoidosis Vasc Diffuse Lung Dis 2019;36 (1):2-10.

Voortman M, Beekman E, Drent M, Hoitsma E, De Vries J. Determination of the smallest detectable change (SDC) and the minimal important change (MIC) for the Small Fiber Neuropathy Screening List (SFNSL) in sarcoidosis.

Sarcoidosis Vasc Diffuse Lung Dis 2018;35:333-341.

Voortman M, Fritz D, Vogels OJM, Eftimov E, van de Beek D, Brouwer MC, Drent M. Small fiber neuropathy: a disabling and underrecognized syndrome.

Curr Opin Pulm Med 2017;23(5):447-457. doi: 10.1097/ MCP.0000000000000413. Review. PMID: 28639956.

Fritz D, Voortman $\mathbf{M}$, van de Beek D, Drent $M$, Brouwer MC. Many faces of neurosarcoidosis: from chronic meningitis to myelopathy.

Curr Opin Pulm Med 2017;23(5):439-446. doi: 10.1097/MCP.0000000000000401. Review. PMID: 28598872 
Mohamed Hoesein FA, Voortman M, Kwakkel-van Erp JM, Luijk B, de Jong PA. Images in COPD: Combined pulmonary emphysema and fibrosis with pulmonary hypertension.

Chronic Obstr Pulm Dis (Miami) 2017;4(1):76-80. doi: 10.15326/jcopdf.4.1.2016.0171. PMID: 28848914

Voortman M, van Ooij PJAM, van Hulst RA, Zanen P. Pulmonary function in professional navy divers and changes during their professional careers.

Undersea Hyperb Med 2016;43(6):649-657. PMID: 28768392

Voortman M, de Jonge N, Ramjankhan FZ, Kwakkel-van Erp JM. Obstructive sleep apnoea in chronic heart failure with near resolution after implantation of a left ventricular assist device.

J Heart Cardiol 2014 (1):1;1-4. doi.org/10.15436/2378-6914.14.0016914.14.001

\section{Contributing author}

Stern BJ, Royal W 3rd, Gelfand JM, Clifford DB, Tavee J, Pawate S, Berger JR, Aksamit AJ, Krumholz A, Pardo CA, Moller DR, Judson MA, Drent M, Baughman RP. Definition and Consensus Diagnostic Criteria for Neurosarcoidosis: From the Neurosarcoidosis Consortium Consensus Group. JAMA Neurol 2018;75(12):1546-1553.doi: 10.1001/jamaneurol.2018.2295. PMID: 30167654

\section{Invited speaker}

2016 (9-12) Vele gezichten van neurosarcoïdose.

Masterclass ild care foundation: Sarcoïdose, diagnostiek en management: een update. Ede, the Netherlands.

2017 (5-4) Neurosarcoïdose en dunne vezelneuropathie.

Landelijke nascholing ILD-verpleegkundigen, St. Antonius Ziekenhuis Nieuwegein, the Netherlands.

2017 (20-5) Consequences of sarcoidosis. Postgraduate course.

American Thoracic Society (ATS) International Conference 2017, Washington, USA.

2019 (20-2) Longgeneeskundig perspectief sarcoidose.

Regionale refereeravond cardiologie UMC Utrecht, the Netherlands

2019 (23-4) Neurosarcoidosis.

Sarcoidosis conference, Belgrade, Serbia. 
2019 (23-4) Small fiber neuropathy.

Sarcoidosis conference, Belgrade, Serbia.

2019 (21-5) Fighting fatigue and other non-specific symptoms.

American Thoracic Society (ATS) International Conference 2019, Dallas, USA.

2019 (22-11) Longgeneeskundig perspectief sarcoïdose

CarVasZ symposium, NVHVV (Nederlandse Vereniging van Hart- en Vaatverpleegkundigen). Ede, the Netherlands.

\section{Patients societies}

2016 (11-11) Neurosarcoïdose.

Sarcoïdosepatiëntendag, St. Antonius Ziekenhuis, Nieuwegein, the Netherlands.

2017 (22-3) De vele gezichten van neurosarcoïdose.

Neurosarcoïdosepatiëntenbijeenkomst: ild care foundation en Sarcoidose.nl. Ede, the Netherlands.

2017 (7-10) Longproblemen bij Sjögren.

Sjögren patiëntenbijeenkomst: Nationale Vereniging Sjögren Patiënten (NVSP). Ede, the Netherlands

2018 (13-10) EGPA: achtergrond en behandeling.

EGPA patiëntenbijeenkomst: Vasculitis stichting. Ede, the Netherlands

2019 (16-3) Vasculitis en de longen.

Vasculitis patiënten bijeenkomst: Vasculitis stichting. Ede, the Netherlands

2019 (18-9) Dunne vezelneuropathie geassocieerde klachten bij sarcoïdose.

Sarcoïdose patiëntenbijeenkomst: Sarcoidose.nl. Utrecht (UMC Utrecht), the Netherlands.

\section{Oral presentations}

2018 (8-6) Interpretability of the small fiber neuropathy screenings list.

World Association of Sarcoidosis and other Granulomatous Disorders (WASOG) 2018 International Conference on sarcoidosis and ILD. Heraklion, Crete, Greece. 


\section{Poster presentations}

2013 (17-5) Obstructive sleep apnoea in chronic heart failure with near resolution after implantation of a left ventricular assist device.

American Thoracic Society International Conference 2013, Philadelphia, USA.

2017 (21-5) Interstitial lung disease in patients with primary Sjogren's syndrome: correlation of radiology, pathology, and clinical phenotype.

American Thoracic Society International Conference 2017, Washington, USA. 
Dankwoord 



\section{Dankwoord}

Wat heerlijk om na jaren van hard werken nu mijn dankwoord te mogen schrijven. Het is een mooi avontuur geworden dankzij onderstaande mensen die ik graag zou willen bedanken.

Als eerste natuurlijk prof. dr. Drent. Beste Marjolein. Wat een fantastisch persoon ben jij. Van die eerste meeting op Utrecht Centraal bij de Starbucks in 2016 over een nieuw onderzoeksproject tot nu mijn promotor. Zonder jou was dit nooit gelukt, zeker niet binnen dit tijdsbestek. Als ik jou een nieuwe draft van een manuscript stuurde (en dacht even rust te hebben), kreeg ik het vaak dezelfde dan wel de volgende dag weer retour met opmerkingen/aanpassingen. Ik heb je leren kennen als een echte 'duizendpoot', wat jij naast de kliniek en onderzoek allemaal nog als neventaken doet, wow. Jij hebt het vermogen om onderzoek echt naar de patiënt te brengen. 'Wat is nou voor de patiënt echt relevant?' zeg je vaak. De bevlogenheid die jij nog steeds hebt voor je vak, de patiënten en het onderzoek vind ik bewonderingswaardig. Zelfs toen je door ziekte thuis kwam te zitten was je er altijd voor me. Dank voor alles. Op naar een mooie toekomst samen binnen de sarcoïdose wereld en daarbuiten.

Beste prof. dr. De Vries, beste Jolanda. Ondanks dat je zelf heel druk bent, kon ik bij je terecht met mijn (statistische) vragen en was je altijd snel in beantwoorden van mijn mails (meestal na een reminder per app (:) ) met een duidelijke uitleg zodat ik snel verder kon. Je vermogen om een artikel beter neer te zetten qua bewoordingen en het zo aan te passen dat de boodschap duidelijker wordt waardeer ik zeer. Dank dat je mijn $2^{\mathrm{e}}$ promotor wil zijn.

Geachte leden van de beoordelingscommissie: prof. dr. F.W.J.M. Smeenk, prof. dr. R.W.H.M. Ponds, prof. dr. A. Bast, prof. dr. J.C. Grutters, prof. dr. A. Dahan. Hartelijk dank voor het lezen en beoordelen van het manuscript.

Beste prof. dr. Grutters, beste Jan. Ik herinner me nog goed ons eerste gesprek in een weekenddienst tijdens mijn opleiding tot longarts in 2014 over mijn interesse in de interstitiële longziekten (ILD). Het ging over mijn wens om klinisch onderzoek binnen dit vakgebied te gaan doen en mijn doel om de ILD samen met jou verder uit te bouwen in het UMC Utrecht. En kijk waar we nu zijn. Jij blijft me altijd verbazen met je tomeloze energie, je vermogen om nieuwe onderzoeksvragen te bedenken en je uitgebreide kennis, zowel klinisch als basaal. Ik ben blij dat je mij de kans hebt gegeven om onderzoek te gaan doen inclusief een fellowship in het UMC Utrecht en het ILD 
Expertisecentrum in het St. Antonius Ziekenhuis Nieuwegein. Het is een plezier en ontzettend leerzaam om nauw met je te mogen samenwerken en jou als mijn 'vraagbaak' te hebben. Hopelijk zullen we nog vele mooie dingen samen gaan neerzetten. Op naar een vruchtbare verdere samenwerking.

Beste prof. dr. Heijerman, beste Harry. Jij kwam voor mij precies op het juiste moment op de juiste plek, want mijn contract zou aflopen op de dag dat jij begon als hoofd van de Afdeling Longziekten in het UMC Utrecht. Gelukkig kon je alvast even een tijdelijke verlenging regelen op dat moment. Mede dankzij jou heb ik mijn droombaan kunnen bemachtigen. Dank voor je vertrouwen in mij als persoon, als onderzoeker en als arts.

Beste Marjon, vaak sta je in de schaduw van Marjolein, maar ik ben er allang achter dat jij de spin in het web bent voor haar. Dank voor het maken van de online surveys, je hulp bij het omzetten van de data en het corrigeren van mijn artikelen. Dat heeft mij veel werk gescheeld. Daarnaast zou de neurosarcoïdose patiënten dag ook nooit van de grond gekomen zijn zonder jouw hulp. Dus dank voor alles.

Beste dr. Vogels, beste Oscar. Als expert op het gebied van de neurosarcoïdose binnen het ILD Expertisecentrum in het St. Antonius Ziekenhuis heb je mij geholpen met het neurosarcoïdose onderzoek en het prepareren van het manuscript. Veel dank daarvoor.

Beste Daan Fritz, Dr. Matthijs Brouwer en Prof. dr. Diederik van de Beek, dank voor de vruchtbare samenwerking op het gebied van neurosarcoïdose. Het was mooi de door jullie verzamelde patiënten te kunnen koppelen aan onze eigen database. Hierdoor werd het aantal neurosarcoïdosepatiënten verdubbeld. Samen staan we sterker!

Beste Paul, dank voor jouw hulp bij de statistiek in ons kwaliteit van leven onderzoek. Jouw heldere uitleg en snelle respons op mijn geschreven stukken waren super.

Beste Emmylou, dank voor het verrichten van de lastige statische analyse van het onderzoek naar de klinische relevante verandering in de small fiber neuropathy screening list (SFNSL). Het was niet makkelijk, maar gelukkig hebben we het gered $\odot$.

Dr. Elske Hoitsma, jou wil ik bedanken voor het feit dat jij dunne vezelneuropathie (small fiber neuropathy (SFN)) bij sarcoïdose op de kaart hebt gezet. Je hebt de onderliggende problemen erkend en beschreven bij sarcoïdosepatiënten. Dit heeft een enorme impact gehad in de sarcoïdosewereld. Deze erkenning is enorm belangrijk voor de patiënten die het betreft. Ook de SFNSL werd door jou ontwikkeld en wordt inmiddels wereldwijd gebruikt als screening voor SFN-gerelateerde klachten. De aanzet 
die jij hebt gegeven heb ik verder kunnen uitwerken. Ook heb je samen met Esther van Noort en Prof. dr. Marjolein Drent het Neurosarcoïdoseregister opgezet. De gegevens hieruit zijn verwerkt in mijn proefschrift. Zonder jullie initiatief had dat niet gekund. Hartelijk dank daarvoor!

Beste Celine, jou wil ik bedanken voor het verwerken van de survey over orgaangerelateerde en niet-orgaangerelateerde, niet-specifieke klachten bij sarcoïdosepatiënten in Nederland, Duitsland en Denemarken. Dit heeft inzicht verschaft in de impactverschillen en overeenkomsten van sarcoïdosepatiënten in deze Europese landen.

I would like to thank all my international co-authors for their feedback and support in the preparation of some manuscripts. So thank you Prof. Bob Baughman, Prof. Ulrich Costabel, Dr. Francesco Bonella and Dr. Janne Møller.

Beste Petal, jij bent er altijd als ik je nodig heb. Ondanks dat we elkaar nooit in real life gezien hebben, is het altijd leuk je aan de telefoon te hebben. Hoe makkelijk jij je maneuvreert in Endnote en SPSS is bewonderingswaardig. Je hebt me meerdere malen op weg geholpen met onder andere een Endnote spoedcursus (en volgens mij ben ik niet de enige). Dus dank daarvoor, dat heeft het schrijven van de artikelen een stuk makkelijker gemaakt. Daarnaast ben je ook een geweldige fotografe, dank voor je mooie foto op de cover van dit boekje.

Jan Klerkx, veel dank voor het beoordelen en corrigeren van de Engelse tekst van vele hoofdstukken in dit proefschrift. Je correcties waren altijd 'spot-on' en supersnel, dus dank.

Tiny Wouters, bedankt voor al je hulp bij het opmaken van dit proefschrift. Je snelheid van (weer) een nieuwe PDF na aanpassingen van mijn kant is super.

Collegae longartsen en verpleegkundig specialisten: dank voor jullie steun voor dit promotietraject. Ben blij met zulke fijne, betrokken collegae.

Ook wil ik de sarcoïdosepatiëntenvereniging Sarcoidose.nl en alle participerende sarcoïdosepatiënten bedanken uit binnen- en buitenland voor hun deelname aan de verschillende studies. Daarnaast wil ik de sarcoïdosepatiëntenvereniging Sarcoidose.nl ook bedanken voor het toekennen van de Bouwhuisprijs voor mijn onderzoek. Zonder jullie was al dit onderzoek niet mogelijk geweest. 
In het bijzonder wil ik ZonMw bedanken voor het toekennen van een onderzoeksgrant om dit onderzoek mogelijk te maken. Dit heeft mede bijgedragen tot het verschaffen van meer inzicht in allerlei facetten van neurosarcoïdose en dunne vezelneuropathie bij sarcoïdosepatiënten.

Dank ook aan de ild care foundation voor het ondersteunen en mogelijk maken van de onderzoeken in dit proefschrift en het financieel bijdragen aan het promotieboekje.

Daarnaast zou ik graag ook de overige sponsoren willen bedanken voor hun financiële bijdrage voor promotieboekje.

En dan mijn paranimfen.

Lieve Anne, ik heb je leren kennen tijdens onze vooropleiding interne (2009) in het Diakonessenhuis Utrecht en ons pad lijkt veel op elkaar. Tijdens onze opleiding tot longarts was jij twee jaar verder dan ik en ik heb je altijd als mijn voorbeeld gezien als dokter en mens. Zo betrokken, veel kennis, en ambitieus. We zijn goede vriendinnen geworden, samen ons zwangerschapsverlof, gezellige etentjes, altijd sta je voor mij klaar. Jij weet me altijd moed in te praten als het even tegenzit en nu ben je mijn paranimf. Dankbaar dat je me vandaag bijstaat en leuk om dit moment samen te delen!

Lieve Janneke, vanaf dag één op de longafdeling (2008) hadden we al een klik en dat is uitgegroeid tot een hechte vriendschap. Ik vind jou een prachtmens, jij straalt zoveel positiviteit en vrolijkheid uit. Daarnaast vind ik het superknap hoe jij je opgewerkt hebt tot verpleegkundig specialist longoncologie, en niet zomaar één. Menig dokter kan nog veel van je bevlogenheid voor je vak en patiënten, je kennis en empathie leren. Ik ben blij en trots dat je vandaag naast me staat. Heel leuk om dit samen te kunnen doen.

Karin \& Taco, Vivienne, Anne \& Frank, Janneke, Inez, Maarten \& Marloes, Chris \& Aurore, Nicoline \& Ivo, Ruud \& Daphne, Michiel \& Jacqueline, Bas \& Trine, Jo \& Anneleen. Lieve vrienden, altijd gezelligheid met heerlijke diners en borrels in binnenen buitenland. Fijn om even geheel uit de werk-/onderzoeksmodus te kunnen komen en over andere dingen te kunnen praten en nadenken. Ik ben dankbaar voor zulke lieve/leuke vrienden.

En dan mijn familie.

Lieve Paul en mama, dankzij jullie opvoeding sta ik hier vandaag. Jullie leerde me dat goed je best doen belangrijk is en dat je het niet voor niks krijgt. Door die instelling sta ik hier nu. Dank dat jullie altijd voor mij en mijn gezin klaarstaan. Heerlijke weekenden ontspannen en ontstressen in Ambt Delden op de boerderij of even voor onze kinderen 
zorgen zodat ik tijd had voor mijn onderzoek of dat Mark en ik even tijd hadden om samen te ontspannen. Dank jullie wel voor alles.

Lieve papa en Wilma, tja de (overdreven) werklust heb ik zeker van jou geërfd pap (:). Dank ook aan jullie voor een luisterend oor en oppasdagen/weekenden daar in het Hoge Noorden. Ondanks dat we elkaar minder zien vanwege de afstand, ben ik blij dat jullie er voor ons zijn.

Lieve Laurens en Anette. Ik kon niet meer geboft hebben met mijn schoonouders. Jullie zijn zulke lieve mensen en staan altijd voor ons klaar. Hoe vaak jullie wel niet opgepast hebben bij ons in Utrecht vanwege werkverplichtingen of congressen, of even oppassen zodat Mark en ik samen op reis konden om even te ontspannen. Zonder jullie had ik dit nooit gered!

Bart, broertje, helaas zien we elkaar te weinig maar altijd als we elkaar zien is het gezellig.

Patti, zussie, we zien elkaar niet zoveel doordat jij letterlijk aan de andere kant van de wereld aan het promoveren bent. Ik wens je heel veel succes, you're next!!

En last but definitely not least, mijn man Mark en onze kids Sofia en Hugo. Zonder jou Mark, had ik dit nooit gekund. Jij bent zo'n ontzettend positief persoon die altijd het volste vertrouwen in mij heeft gehad. Als ik twijfelde (promotie, longarts in de kliniek en een jong gezin) was jij ervan overtuigd dat ik dat wel kon. Met jou kan ik overal over praten, en meerdere keren heb jij mij geholpen mijn dromen en ambities waar te maken. Jouw adviezen zijn goud waard. En ondanks dat je een leuke en goede baan hebt, heb je je eigen ambities toch wel even op de wat langere baan geschoven voor mij. Daar ben ik je eeuwig dankbaar voor. Hou ontzettend veel van je.

En onze lieve kids, Sofia en Hugo. Jullie waren en zijn altijd mijn baken geweest. Thuis kon ik van jullie genieten, genieten van die heerlijke gedachtegangen die alleen kinderen kunnen hebben. Het is zo leuk om jullie te zien opgroeien. Het geeft het leven weer een totaal andere wending. Dank voor jullie geduld als mama 'weer' aan het werk moest gaan. Ik hou van jullie. 

Curriculum vitae 



\section{Curriculum vitae}

Mareye Voortman was born on April 8th, 1984 in Hengelo (Province of Overijssel), the Netherlands. From 1996 until 2002 she attended secondary school (Atheneum) at the Openbare Scholengemeenschap Bataafse Kamp in Hengelo. After graduation, she attended Medical School at the University of Utrecht from 2002 until 2008. During her medical training, she completed an elective clinical internship on tropical medicine at Huruma District Hospital, Tanzania. In 2009 she started training as a pulmonologist at the Department of Pulmonology of the University Medical Centre Utrecht (UMCU), the Netherlands. Since 2016 she has been working as a

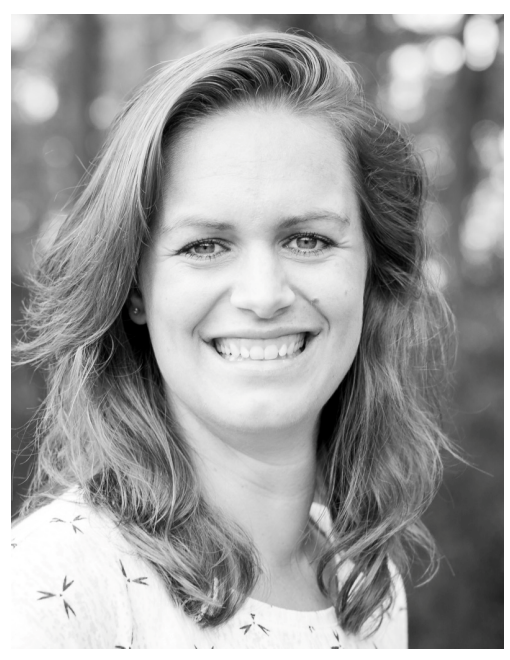
pulmonologist (ILD-fellowship) and since January 2018 she has been a staff member of the Department of Pulmonology at UMCU. She is a member of the ild care foundation research team, taking a special interest in interstitial lung diseases (ILD) related to systemic diseases and sarcoidosis. In 2016 she started a research project at the ILD Centre of Excellence at St. Antonius Hospital, Nieuwegein, the Netherlands, entitled: 'Unraveling neurosarcoidosis in the Netherlands: prevalence, diagnostic work-up and treatment' (ZonMW grant; 842002005). Results of this and other research projects are reported on in this thesis. Prof. Marjolein Drent and Prof. Jolanda De Vries are her supervisors. The results of the studies so far were presented at the WASOG (World Association of Sarcoidosis and Other Granulomatous Disorders) 2018 International Conference in Crete (Greece) in June 2018, the Sarcoidosis Convention Belgrade in April 2019 (invited speaker) and at the American Thoracic Society (ATS) conference in Dallas, USA, in May 2019 (invited speaker). Furthermore, she has also given a presentation at the postgraduate course Sarcoidosis at the ATS conference in Washington, USA, in May 2017. In 2019 she was awarded the Bouwhuis award for PhD students by the Dutch Sarcoidosis Society (Sarcoidose.nl).

She lives in Utrecht with her husband, Mark Gering, and their two kids, Sofia (2011) and Hugo (2014). 

Abbreviations 


\section{Abbreviations}

ACE

ALS

ANOVA

APC

AUC

AZA

$\beta$

BAL

CAFT

CCM

CES-D

CFQ

CHEP

$\mathrm{Cl}$

CIPD

CNFD

CNS

COPD

CSF

CT

CXCR4

DN

EBUS-FNA

EMG

FAS

FDG-PET-CT

GE

HADS

HIV

HLA

HRCT

IENFD

IFN- $\gamma$

IL

ILD angiotensin converting enzyme

amyotrophic lateral sclerosis

analysis of variance

antigen presenting cells

area under curve

azathioprine

standardized regression coefficients

bronchoalveolar lavage

cardiovascular autonomic testing

corneal confocal microscopy

center for epidemiological studies-depression scale

cognitive failure questionnaire

contact heat-evoked potential

$95 \%$ confidence interval

chronic inflammatory demyelinating polyradiculoneuropathy

corneal nerve-fiber density

central nervous system

chronic obstructive pulmonary disease

cerebrospinal fluid

computer tomography

$\mathrm{C}-\mathrm{X}$-C motif chemokine receptor 4

Denmark

endobronchial ultrasound fine-needle aspiration

electromyography

fatigue assessment scale

fluordoexyglucose positron emission tomography-computer tomography

Germany

hospital anxiety and depression scale

human immunodeficiency virus

human leukocyte antigen

high resolution computed tomography

intraepidermal nerve fiber density

interferon-gamma

interleukin

interstitial lung disease 


\begin{tabular}{|c|c|}
\hline KSQ & King's sarcoidosis questionnaire \\
\hline KvL & kwaliteit van leven \\
\hline LEP & laser-evoked potential \\
\hline MCID & minimal clinical important difference \\
\hline $\mathrm{MDC}_{95}$ & minimal detectable change when using its $95 \%$ confidence interval \\
\hline $\mathrm{MHC}$ & major histocompatibility complex \\
\hline MIBG-scan & metaiodobenzylguanidine-scan \\
\hline MID & minimal important difference \\
\hline MMF & mycophenolate mofetil \\
\hline MRI & magnetic resonance imaging \\
\hline MS & multiple sclerosis \\
\hline MTX & methotrexate \\
\hline $\mathrm{n}$ & number \\
\hline NCS & nerve conduction study \\
\hline NL & the Netherlands \\
\hline NPV & negative predictive value \\
\hline NS & neurosarcoidosis \\
\hline NS & non-significant \\
\hline NSAID & non-steroidal anti-inflammatory drug \\
\hline $\mathrm{NI}$ & olfactory nerve \\
\hline NII & optic nerve \\
\hline NIII & oculomotor nerve \\
\hline NIV & trochlear nerve \\
\hline NV & trigeminal nerve \\
\hline NVI & abducens nerve \\
\hline NVII & facial nerve \\
\hline NVIII & vestibulocochlear nerve \\
\hline NIX & glossopharyngeal nerve \\
\hline NX & vagus nerve \\
\hline NXI & accessory nerve \\
\hline NXII & hypoglossal nerve \\
\hline OR & odds ratio \\
\hline $\mathrm{p}$ & $\mathrm{p}$-value \\
\hline PREP & pain-related evoked potential \\
\hline PROMs & patient-related outcomes \\
\hline PSSS & perceived social support scale \\
\hline QoL & quality of life \\
\hline QSART & quantitative sudomotor axon reflex test \\
\hline
\end{tabular}




$\begin{array}{ll}\text { QST } & \text { quantitative sensory testing } \\ \text { R } & \text { Pearson correlation coefficient } \\ \text { R }^{2} & \text { R-square change of the model } \\ \text { RCI } & \text { repository corticotropin injection } \\ \text { ROC } & \text { receiver operating characteristic } \\ \text { SD } & \text { standard deviation } \\ \text { SDC } & \text { smallest detectable change } \\ \text { SDS } & \text { sarcoidosis diagnostic score } \\ \text { SEM } & \text { standard error of measurement } \\ \text { SFN } & \text { small fiber neuropathy } \\ \text { SFNSL } & \text { small fiber neuropathy screening list } \\ \text { SIADH } & \text { syndrome of inappropriate antidiuretic hormone } \\ \text { SIL2R } & \text { soluble interleukin-2 receptor } \\ \text { SPSS } & \text { statistical package for the social sciences } \\ \text { SSFN } & \text { sarcoidosis small fiber neuropathy } \\ \text { SSNRI } & \text { selective serotonin norepinephrine reuptake inhibitors } \\ \text { SSR } & \text { sympathetic skin response } \\ \text { STAI } & \text { state and trait anxiety inventory } \\ \text { SVR } & \text { skin vasomotor reflex } \\ \text { TBLB } & \text { transbronchial lung biopsy } \\ \text { TCA } & \text { tricyclic antidepressant } \\ \text { Th1 } & \text { type } 1 \text { helper } \\ \text { TNF- } \alpha & \text { tumour necrosis factor-alpha } \\ \text { TSH } & \text { thyroid stimulating hormone } \\ \text { TST } & \text { thermoregulatory sweat test } \\ \text { TTT } & \text { visual analogue scale } \\ \text { UMC } & \text { World Association of Sarcoidosis and Other Granulomatous Disorders } \\ \text { VAS } & \text { WASOG }\end{array}$



Appendix questionnaires 



\section{Fatigue Assessment Scale}

The Fatigue Assessment Scale (FAS; Table A.1) is a 10-item self-report fatigue questionnaire. $^{1,2}$ It is quick and easy to complete for patients, and not time consuming. An answer to every question has to be given, even if the person does not have any complaints at the moment. Five questions reflect physical fatigue and 5 questions (questions 3 and 6-9) mental fatigue. The response scale is a five-point scale (1 never to 5 always). Subsequently, the total FAS score can be calculated by summing the scores on all questions (recoded scores for questions 4 and 10). Scores on the FAS can range from 10 to 50 . A score $>22$ indicates fatigue and a score $>34$ indicates extreme fatigue. Scores on question 4 and 10 should be recoded $(1=5,2=4,3=3,4=2,5=1)$. The reliability and validity of the FAS have been shown to be good in sarcoidosis patients. ${ }^{1}$ So far, the FAS is available in 20 languages (see http://www.wasog.org/educationresearch/questionnaires.html). ${ }^{3}$

The Minimal Important Difference (MID) is at least 4 points or $10 \%$ change of the baseline value. ${ }^{4}$

\section{Table A.1 Fatigue Assessment Scale (FAS).}

The following ten statements refer to how you usually feel. Per statement you can choose one out of five answer categories, varying from Never to Always. Please circle the answer to each question that is applicable to you. Please give an answer to each question, even if you do not have any complaints at the moment.

1. never

2. sometimes (about monthly or less)

3. regularly (about a few times a month)

4. often (about weekly)

5. always (about every day)

\begin{tabular}{|c|c|c|c|c|c|c|}
\hline & & never & sometimes & regularly & often & always \\
\hline 1. & I am bothered by fatigue & & & & & \\
\hline 2. & I get tired very quickly & & & & & \\
\hline 3. & I don't do much during the day & & & & & \\
\hline 4. & I have enough energy for every day life & & & & & \\
\hline 5. & Physically, I feel exhausted & & & & & \\
\hline 6. & I have problems to start things & & & & & \\
\hline 7. & I have problems to think clearly & & & & & \\
\hline 8. & I feel no desire to do anything & & & & & \\
\hline 9. & Mentally, I feel exhausted & & & & & \\
\hline 10. & $\begin{array}{l}\text { When I am doing something, I can } \\
\text { concentrate quite well }\end{array}$ & & & & & \\
\hline
\end{tabular}




\section{Small Fiber Neuropathy Screenings List}

The small fiber neuropathy screenings list (SFNSL; Table A.2) was developed to assess symptoms which may be related to SFN, but not to diagnose SFN. The SFNSL is a 21-item self-administered questionnaire to screen for symptoms related to SFN. The response scale is a five-point scale ( 0 never to 4 always); scores on the SFNSL can range from 0 to 84. The cut-off score of the SFNSL is 11: a score below 11 indicates no or few symptoms related to SFN, while a score of 11-48 indicates probable or highly probable SFN and a score above 48 is indicative of SFN. ${ }^{5}$ So far, the SFNSL is available in six languages; Danish, Dutch, English, French, German, Italian and Japanese (see http://www.wasog.org/education-research/questionnaires.html).

The minimal important difference (MID) on the SFNSL is 3.5 points for a clinically relevant change over a 6-month period. ${ }^{6}$

\section{Table A.2 Small Fiber Neuropathy Screenings List.}

Below are a number of questions about possible complaints. Please circle the answer to each question that is applicable to you. Please give an answer to each question, even if you do not have any complaints at the moment. The aim of this questionnaire is to find out how you experience your complaints. There are no correct or incorrect answers. It is important that you are honest.

Part 1: These questions are aimed at finding out how often you experience the following complaints.

1. I have painful arms

2. I suffer from palpitations

3. I have problems with my bowel movements

4. I have difficulties with urinating (either in emptying my bladder or being able to hold my water)

5. My food does not seem to go down well

6. I suffer from muscle cramps

7. My feet and/or hands are colder than I am used to

8. I have chest pain

Part 2: These questions are aimed at finding out how serious your complaints are. not at all slightly variably moderately seriously

9. I have the feeling that my food gets stuck in my throat

10. At night I throw the bedclothes off my legs

11. I have difficulties with urinating (either emptying my bladder or being able to hold my water)

12. I have dry eyes

13. I have blurred vision

14. I feel dizzy when I get up

15. I have sudden hot flushes

16. My feet and/or hands are colder than I am used to

17. I have painful arms

18. The skin of my legs is over-sensitive

19. I have a tingling sensation in my hands (pins and needles)

20. I have a tingling sensation in my legs (pins and needles)

21. I have chest pain 


\section{The Cognitive Failure Questionnaire}

The Cognitive Failure Questionnaire (CFQ, table A3) is a 25-item self-report questionnaire assessing failures in everyday errors of attention, perception, memory and motor function in everyday life. ${ }^{7}$ In general, the CFQ appears to be a reliable and brief measure useful in clinical practice. The response scale is a five-point scale ( 0 never to 4 very often). The total CFQ score is calculated by summation of all answers and scores range from 0-100. A higher total score indicates more subjective cognitive failure. A high CFQ score is defined as a score $\geq 43$ (mean of the controls plus one standard deviation). ${ }^{7}$

Table A.3 Cognitive Failure Questionnaire.

The following questions are about minor mistakes which everyone makes from time to time, but some of which happen more often than others. We want to know how often these things have happened to you in the past 6 months. 
1. Do you read something and find you haven't been thinking about it and must read it again?

2. Do you find you forget why you went from one part of the house to the other?

3. Do you fail to notice signposts on the road?

4. Do you find you confuse right and left when giving directions?

5. Do you bump into people?

6. Do you find you forget whether you've turned off a light or a fire or locked the door?

7. Do you fail to listen to people's names when you are meeting them?

8. Do you say something and realize afterwards that it might be taken as insulting?

9. Do you fail to hear people speaking to you when you are doing something else?

10. Do you lose your temper and regret it?

11. Do you leave important letters unanswered for days?

12. Do you find you forget which way to turn on a road you know well but rarely use?

13. Do you fail to see what you want in a supermarket (although it's there)?

14. Do you find yourself suddenly wondering whether you've used a word correctly?

15. Do you have trouble making up your mind?

16. Do you find you forget appointments?

17. Do you forget where you put something like a newspaper or a book?

18. Do you find you accidentally throw away the thing you want and keep what you meant to throw away - as in the example of throwing away the matchbox and putting the used match in your pocket?

19. Do you daydream when you ought to be listening to something?

20. Do you find you forget people's names?

21. Do you start doing one thing at home and get distracted into doing something else (unintentionally)?

22. Do you find you can't quite remember something although it's "on the tip of your tongue"?

23. Do you find you forget what you came to the shops to buy?

24. Do you drop things?

25. Do you find you can't think of anything to say?

Copyright Broadbent. ${ }^{7}$ 


\section{References}

1. De Vries J, Michielsen $H$, Van Heck GL, Drent $M$. Measuring fatigue in sarcoidosis: the Fatigue Assessment Scale (FAS). Br J Health Psychol 2004;9(Pt 3):279-291.

2. Drent M, Lower EE, De Vries J. Sarcoidosis-associated fatigue. Eur Respir J 2012;40(1):255-263.

3. Hendriks C, Drent M, Elfferich M, De Vries J. The Fatigue Assessment Scale: quality and availability in sarcoidosis and other diseases. Curr Opin Pulm Med 2018;24(5):495-503.

4. Kleijn WPE, De Vries J, Wijnen PAHM, Drent M. Minimal (clinically) important differences for the Fatigue Assessment Scale in sarcoidosis. Respir Med 2011;105:1388-1395.

5. Hoitsma E, De Vries J, Drent M. The small fiber neuropathy screening list: construction and crossvalidation in sarcoidosis. Respir Med 2011;105(1):95-100.

6. Voortman M, Beekman E, Drent M, Hoitsma E, De Vries J. Determination of the smallest detectable change (SDC) and the minimal important change (MIC) for the Small Fiber Neuropathy Screening List (SFNSL) in sarcoidosis. Sarcoidosis Vasc Diffuse Lung Dis 2018;35;333-341.

7. Broadbent DE, Cooper PF, Fitzgerald P, Parkes KR. The cognitive failures questionnaire (CFQ) and its correlates. Br J Clin Psychol 1982;21(Pt1):1-16. 
